Universidade de São Paulo

Instituto de Física

\title{
CONTROLE DA TURBULÊNCIA EM PLASMAS
}

\section{DENNIS LOZANO TOUFEN}

Tese de doutorado apresentada ao Instituto de Física para a obtenção do título de Doutor em Ciências.

Orientador: Prof. Dr. Iberê Luiz Caldas

Banca Examinadora:

Prof. Dr. Iberê Luiz Caldas (IFUSP)

Prof. Dr. Maria Vittoria A. P. Heller (IFUSP)

Prof. Dr. Yuri Kuznetsov (UEK)

Prof. Dr. Felipe Barbedo Rizzato (UFRGS)

Prof. Dr. Antonio Marcos Batista (UEPG) 


\section{FICHA CATALOGRÁFICA}

Preparada pelo Serviço de Biblioteca e Informação

do Instituto de Física da Universidade de São Paulo

Toufen, Dennis Lozano

Controle da turbulência em plasmas. - São Paulo, 2012.

Tese (Doutorado) - Universidade de São Paulo.

Instituto de Física - Depto. de Física Aplicada

Orientador: Prof. Dr. Iberê Luiz Caldas

Área de Concentração: Física

Unitermos: 1. Física de Plasmas; 2. Física Experimental;

3.Turbulência Eletrostática; 4. Caos (Sistemas Dinâmicos);

5. Fenômenos de Transporte. 
"Eu não sou eu nem sou o outro, Sou qualquer coisa de intermédio: Pilar da ponte de tédio Que vai de mim para o Outro." (Mário de Sá-Carneiro)

"Para ser grande, sê inteiro: nada Teu exagera ou exclui. Sê todo em cada coisa. Põe quanto és

No mínimo que fazes. Assim em cada lago a lua toda Brilha, porque alta vive."

(Ricardo Reis) 


\section{AGRADECIMENTOS}

Ao meu orientador, Prof. Dr. Iberê Luiz Caldas, por todo seu empenho, dedicação e paciência na orientação deste trabalho e na minha formação como pesquisador.

Aos meus pais, Carlos e Cida, meu muito obrigado por tudo. Tudo o que sou eu devo a vocês.

Ao meu irmão Carlos Toufen Jr. pelo exemplo permanente e amizade inestimável.

À Paula pelo companheirismo, compreensão, amizade e amor.

Ao Prof. Kenneth Gentle e sua equipe, especialmente a Tereza, por permitir que utilizasse o Texas Helimak e por me receberem de braços abertos na Universidade do Texas em Austin. (To Professor Kenneth Gentle and your team, especially Tereza, for allowing me use the Texas Helimak in this work and for welcoming me with open arms in University of Texas at Austin).

Ao Dr. Zwinglio Guimarães-Filho por toda ajuda que me prestou na análise e interpretação dos dados.

Ao Dr. Alberto Marcus pela ajuda nas simulações dos sistemas Hamiltonianos.

À todos os colegas de grupo do IFUSP, entre eles: Júlio, David, Celso, Rafael, Everton e Adriane, pelas discussões sérias sobre Física de plasma e sistemas dinâmicos e pelos momentos de descontração.

À todos os colegas do Campus Guarulhos do IFSP, Professores e Servidores, por acreditarem em mim e no meu trabalho.

Aos amigos do grupo Gama do DFN-IFUSP, por contribuírem tão significantemente na minha formação como Físico e pesquisador.

À todos os professores que, durante minha vida de estudante contribuíram para minha formação.

À pró-reitoria de pós-graduação da USP e a Comissão de pós-graduação do IFUSP pelo apoio financeiro na realização dos estágios na Universidade do Texas e na participação de simpósios e congressos. 


\begin{abstract}
The Texas Helimak is a toroidal confinement of plasma device with helically magnetic field lines and which plasma parameters (like the density and temperature) are similar to the edge and the scrape-off-layer of a tokamak. This thesis analyzed the equilibrium and fluctuations in the Helimak Texas plasma. We present the analysis and interpretation of electrostatic control of turbulence and turbulent particle transport by electrical potential, bias, applied externally. Changes in transport and turbulence caused by the change of external electric potential were investigated in a region of uniform radial gradients plasma. Fluctuations in discharges with positive or zero bias have broadband power spectra and a PDF with an accentuated tail that reveals the intermittent occurrence of extreme events. Moreover, fluctuations in discharges with negative bias have narrow power spectra, a more Gaussian PDF and more structures in the recurrence diagram. A four coupled wave model was used to relate the bandwidth of turbulence with the interaction time between the waves in the model. Radial profiles of turbulent transport of particles in the radial direction were calculated as a function of bias and compared to the profiles of the plasma flow velocity and its shear. It was shown that the transport depends on the radial profile of the plasma flow velocity. In that discharges where the velocity radial profile presents a point of maximum, the transport profile has a minimum which was interpreted, using a drift wave model Hamiltonian, as a result of a shearless flow transport barrier. In other discharges, the maxima in the transport radial profiles were related with resonances that occur where the wave phase velocity is equal to the plasma velocity.
\end{abstract}




\section{RESUMO}

O Texas Helimak é uma máquina toroidal de confinamento de plasma cujas linhas de campo magnético têm forma helicoidal e no qual parâmetros do plasma (como a densidade e a temperatura) são similares aos da borda e da região externa (scrape-offlayer) de um tokamak. Nesta tese foram analisados o equilíbrio e as flutuações do plasma no Texas Helimak. São apresentadas a análise e a interpretação do controle da turbulência eletrostática e do transporte turbulento de partículas pelo potencial elétrico, bias, aplicado externamente. As alterações na turbulência e no transporte causadas pela mudança do potencial elétrico externo foram investigadas em uma região do plasma com gradientes radiais uniformes. As flutuações em descargas com bias positivo ou nulo apresentam espectros de potência de banda larga e uma PDF com uma cauda acentuada que revela a ocorrência intermitente de eventos extremos. Por outro lado, as flutuações em descargas com bias negativo possuem um espectro de potência mais estreito, uma PDF mais Gaussiana e um diagrama de recorrência com mais estruturas. Um modelo de quatro ondas acopladas foi utilizado para relacionar a largura de banda da turbulência com o tempo de interação entre as ondas do modelo. Perfis radiais do transporte turbulento de partículas na direção radial foram calculados, como função do bias, e comparados com os perfis da velocidade do fluxo de plasma e seu cisalhamento. Foi mostrado que o transporte depende do perfil radial da velocidade de fluxo do plasma. Nas descargas em que essa velocidade apresenta um ponto de máximo em seu perfil radial, o perfil do transporte possui um mínimo que foi interpretado, utilizando um modelo Hamiltoniano de ondas de deriva, como sendo resultado de uma barreira de transporte onde o cisalhamento do fluxo é nulo. Em outras descargas, máximos no perfil radial do transporte foram relacionados a ressonâncias que ocorrem onde a velocidade de fase da onda é igual à velocidade do plasma. 


\section{SUMÁRIO}

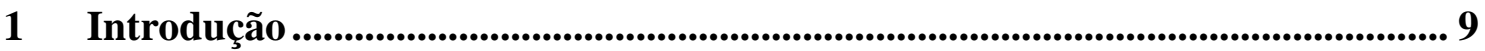

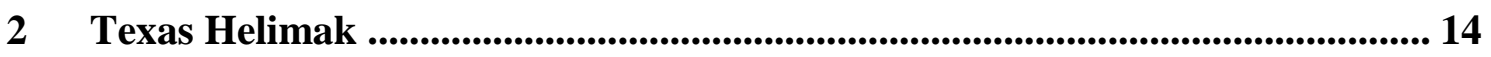

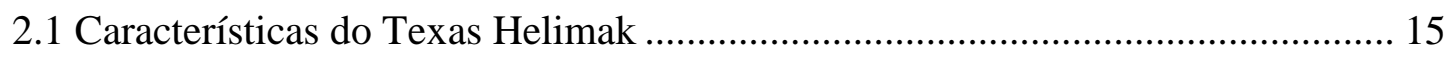

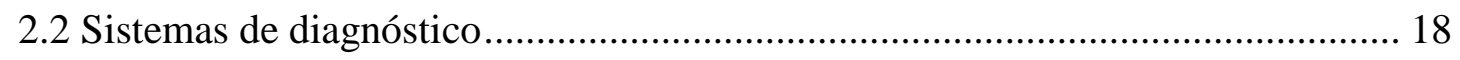

2.3 Existência de equilíbrio e estabilidade MHD no Texas Helimak......................... 21

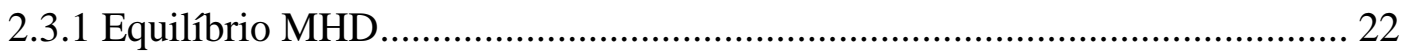

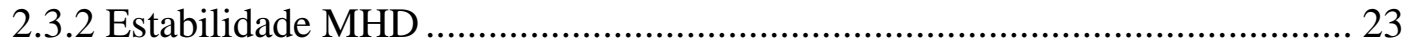

2.4 Imposição de potencial elétrico externo: bias ....................................................... 26

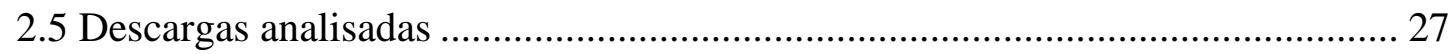

3 Métodos de análise.................................................................................................................. 28

3.1 Análise estatística das flutuações ................................................................. 28

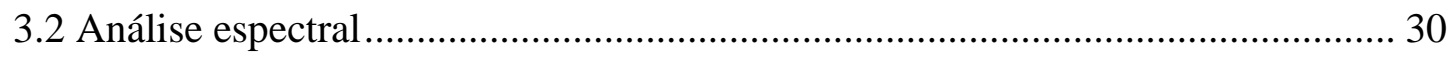

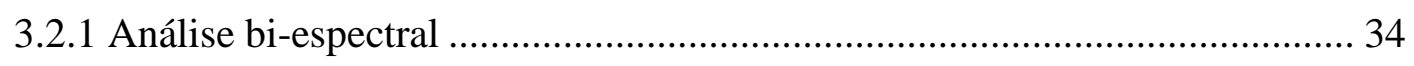

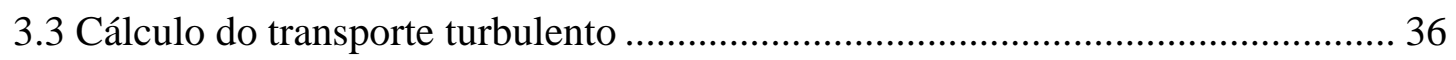

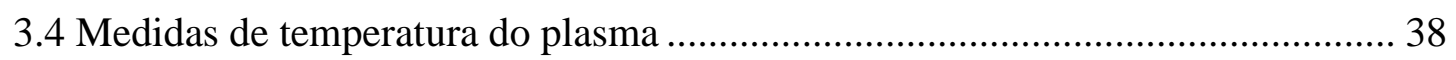

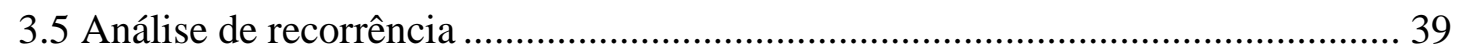

3.6 Medidas de velocidade de fluxo vertical do plasma $\boldsymbol{V} \mathbf{z}$.................................. 42

4 Caracterização do plasma não perturbado no Texas Helimak ......................... 44

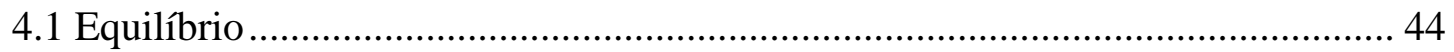

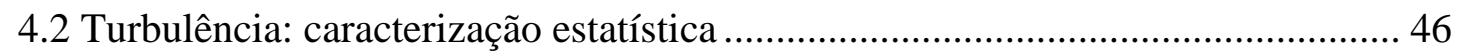

4.3 Turbulência: caracterização espectral ............................................................... 48

4.3.1 Escolha da faixa radial de análise .............................................................. 48

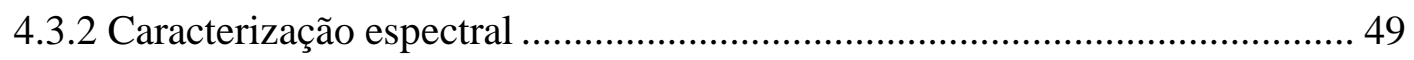

4.4 Considerações finais acerca das características do plasma não perturbado .......... 54

5 Controle através do bias externo ........................................................................56 


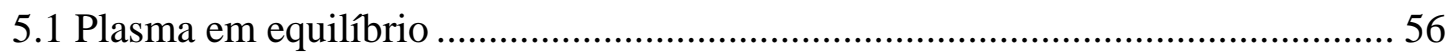

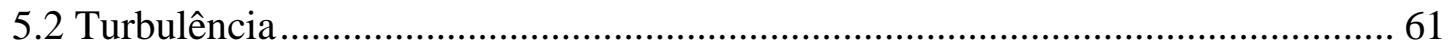

5.2.1 Caracterização estatística da turbulência ..................................................... 61

5.2.2 Caracterização espectral da turbulência ...................................................... 63

5.3 Considerações finais acerca do controle através do bias externo .......................... 71

6 Alterações da turbulência provocadas pelo bias externo .................................... 73

6.1 Influência do bias nas características espectrais gerais da turbulência ................ 74

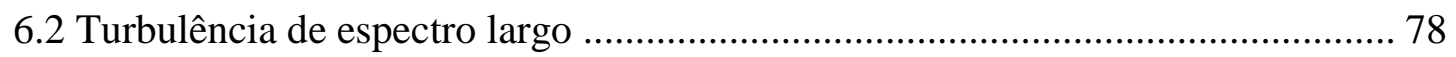

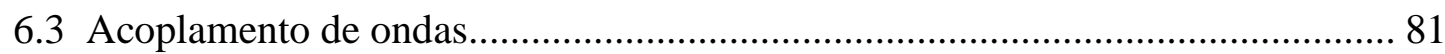

6.3.1 Interpretação teórica: modelo de quatro ondas acopladas ............................ 83

6.4 Análise do modo presente para bias em torno de -16V ....................................... 89

6.5 Considerações finais acerca das alterações da turbulência provocadas pelo bias

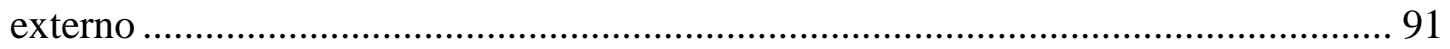

7 Controle do transporte radial de partículas...................................................93

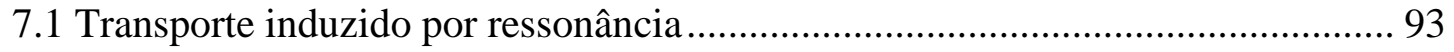

7.1.1 Interpretação teórica: transporte caótico na ressonância ............................... 96

7.2 Evidência de barreira de transporte de cisalhamento nulo (shearless)................ 100

7.2.1 Interpretação teórica: barreiras de cisalhamento zero devidas aos perfis não

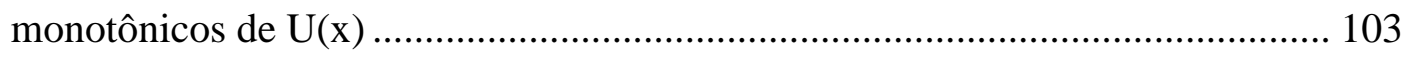

7.3 Considerações finais acerca do controle do transporte radial de partículas ....... 105

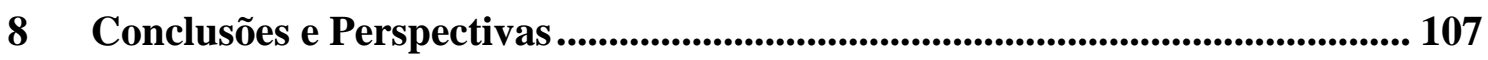

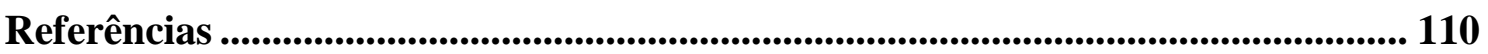

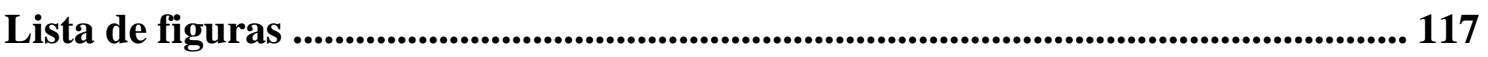

Apêndice I: Tabela de disparos utilizados................................................................... 125 


\section{INTRODUÇÃO}

Nas últimas décadas tem-se investigado o confinamento magnético de plasma em tokamaks e stellarators com o objetivo de viabilizar tecnicamente um reator de fusão termonuclear controlada para a produção de energia elétrica (MEADE, 2010). Processo pelo qual as estrelas produzem energia, a fusão consiste na conversão de núcleos pequenos em núcleos maiores, liberando grande quantidade de energia no processo (FREIDBERG, 2007). Como o combustível nuclear e os produtos da reação dos futuros reatores serão compostos por núcleos leves que não decaem em núcleos radioativos, como Deutério, Trítio e Hélio (PITTS, 2006), a fusão é um processo mais limpo do que a fissão para a obtenção de energia nuclear.

O grande desafio da produção de energia através da fusão nuclear está na pressão e temperatura (em torno de $1,5 \times 10^{8} \mathrm{~K}$ para um futuro reator) necessárias para que a energia cinética dos núcleos seja suficiente para vencer a energia potencial repulsiva (Coulombiana) que separa dois núcleos devido a sua carga positiva (HIDALGO, 2004). Devido a esta alta temperatura que o combustível nuclear deve atingir, os elétrons do material serão separados dos núcleos durante o aquecimento, assim a fusão sempre ocorrerá com o material na forma de plasma (THEILER, 2011).

Sendo assim, a viabilização de um reator de fusão está diretamente ligada a sua capacidade de confinar por tempo suficiente o plasma na pressão e temperatura necessários. Um dos problemas deste confinamento é a perda excessiva de partículas na borda do plasma magneticamente confinado (HAZELTINE, 2002). Essa perda está relacionada com a turbulência apresentada pelo plasma na região de borda (HIDALGO, 1995).

Devida a sua ligação com o transporte de partículas e de calor (GARBET, 2004), a turbulência na borda do plasma tem sido amplamente estudada (GONÇALVES, 2006), tanto em relação aos seus métodos de medida (CONWAY, 2008) quanto às suas abordagens teóricas e simulações (GARBET, 2001).

Uma possibilidade de controle desta turbulência e conseqüente redução do transporte é através da alteração do perfil do campo elétrico radial, o que pode ser conseguido, por exemplo, com a utilização de um eletrodo para controlar o potencial elétrico na região de borda (NASCIMENTO, 2005; VAN OOST, 2003 e HIDALGO, 2003). 
Utilizando o tokamak TCABR e seu antecessor, o Tokamak TBR-1, o grupo do Laboratório de Plasmas do IFUSP tem produzido diversos trabalhos ligados à instrumentação como o desenvolvimento de sondas elétricas (DA SILVA, 1986 e 1991) e a análise da turbulência e do transporte de partículas (CASTRO, 1996; HELLER, 1995 e FERREIRA, 2000). Alguns desses trabalhos recentes abordam a relação da turbulência com a atividade MHD (NASCIMENTO, 2007; HELLER, 2005; HELLER, 2007; DOS SANTOS LIMA, 2009-2 e GUIMARÃES-FILHO, 2008-1).

Máquinas que estudam a física básica de plasmas confinados magneticamente têm se mostrado importantes no estudo da turbulência e do transporte (CARTER, 2009). Estes equipamentos, cuja topologia das linhas do campo magnético é diferente da dos tokamaks, também tem se destacado no desenvolvimento de diagnósticos e no teste de novos modelos para a turbulência (DAHLBURG, 2009; FASOLI, 2006; RYPDAL, 2004 e GEKELMAN, 1991).

Dentre as máquinas de plasma básico, os tóros magnetizados simples (simple magnetized tori - SMT), máquinas nas quais o plasma tem forma toroidal, se destacam principalmente por apresentar uma grande região radial cujas características (de densidade e temperatura) do plasma são similares às da borda e da região externa (scrape-off layer ou SOL) de um tokamak (RICCI, 2008). Os principais SMT atualmente em operação são o TORPEX (FASOLI, 2006), o BLAAMANN (RYPDAL, 2004) e o Texas Helimak (GENTLE, 2008), sendo essas três máquinas também chamadas de helimaks.

Em um helimak o campo magnético é helicoidal: um campo principal toroidal somado a um campo vertical menos intenso (ZIMMERMAN, 1993). Dessa forma as linhas de campo magnético em um helimak circulam em torno do eixo principal formando helicóides. Essa configuração permite um plasma com equilíbrio MHD, gradiente radial de densidade e campo magnético com curvatura e cisalhamento (shear). Devido às características de equilíbrio e baixa temperatura do plasma, a configuração helimak permite ainda o uso de conjuntos com muitas sondas de Langmuir (MÜLLER, 2005).

Apesar de apresentarem estabilidade MHD, os helimaks podem apresentar instabilidades eletrostáticas provocadas pelo gradiente de densidade, pela curvatura do campo magnético e seu cisalhamento (shear), dos tipos: Raleigh-Taylor (interchange instability), Kelvin-Helmholtz, e de ondas de deriva (drift wave instabilities) (GENTLE, 
2010; RYPDAL, 2005; PEREZ, 2006). Estas instabilidades devem ser a causa da turbulência observada nestas máquinas.

Entre os SMT, o Texas Helimak tem como um dos seus diferenciais a capacidade de modificar o perfil do campo elétrico radial através da imposição de um potencial elétrico externo (bias), de forma similar ao que é feito através de um eletrodo em tokamaks (NASCIMENTO, 2005). Essa capacidade permite investigar a influência do perfil radial do campo elétrico na turbulência e no transporte de partículas. Além disso, o Texas Helimak conta com um espectroscópio para medir diretamente a velocidade de fluxo do plasma na direção vertical.

Neste trabalho serão analisados dados do Texas Helimak da Universidade do Texas em Austin (GENTLE, 2008) sobre o plasma confinado e suas oscilações turbulentas, obtidos por um conjunto de sondas de Langmuir e um espectroscópio de deslocamento Doppler.

Esses dados foram adquiridos pela equipe do Prof. Kenneth Gentle e pelo autor desta tese nos dois estágios realizados por ele, em janeiro de 2011 e janeiro de 2012, com o apoio financeiro da Pró-Reitoria de Pós-Graduação da USP. A utilização dos dados nesta tese acontece, portanto, dentro da colaboração estabelecida entre o grupo do Prof. Gentle, da Univ. do Texas e o Grupo de Controle de Oscilações do Departamento de Física Aplicada do IFUSP.

Utilizando o Texas Helimak, instabilidades de ondas de deriva foram identificadas e caracterizadas (PEREZ, 2006). Nesta máquina também foram realizados experimentos que demonstram como a imposição de um potencial elétrico externo pode causar grande redução do nível de turbulência (LI, 2011). Medidas espectroscópicas da velocidade de fluxo do plasma foram usadas para verificar a redução do nível de turbulência com o aumento do cisalhamento da velocidade (GENTLE, 2010), como previsto pelo modelo descrito por TERRY (2000). Essa previsão não foi verificada, ou seja, o modelo de redução da turbulência e do transporte de partículas através do cisalhamento da velocidade não é adequado para descrever a turbulência e o transporte no Texas Helimak.

O objetivo principal desta tese foi investigar e interpretar o controle da turbulência e do transporte turbulento de partículas, no Texas Helimak, como função do potencial elétrico imposto externamente (bias). Essa investigação foi realizada em uma região específica do plasma, no intervalo radial $1,10 \mathrm{~m}<\mathrm{R}<1,25 \mathrm{~m}$, escolhida por 
apresentar um gradiente de densidade aproximadamente constante e por ser a região mais afetada pelo bias.

Para estudo e interpretação das características da turbulência e sua relação com o potencial imposto externamente (bias), foram utilizadas análises estatística, espectral e de recorrência para mostrar que, na região radial analisada a turbulência tem características distintas de acordo com o sinal do bias imposto.

Descargas com bias negativo possuem um espectro de potência mais estreito e de menor potência total, além de apresentarem uma PDF mais Gaussiana e um diagrama de recorrência com mais estruturas. A bicoerência foi calculada para mostrar que a largura de banda do espectro de potência está relacionada ao acoplamento de modos. Para interpretar esse tipo de descarga foi utilizado um modelo de quatro ondas acopladas (LASHMORE-DAVIES, 2005) que inclui o fluxo de plasma (zonal flow) e uma onda dominante. Utilizando valores experimentais para determinar os parâmetros do modelo, o tempo de interação entre as ondas foi estimado como função da posição radial e do bias. Este tempo de interação entre a onda principal e o fluxo de plasma (durante o qual são geradas duas ondas secundárias) foi então comparado com a largura espectral. O que se observa é que tempos de interação curtos (longos) estão ligados a larguras espectrais maiores (menores). A conjectura que se fez é que, tempos de interação curtos permitem uma cascata de interações entre o fluxo de plasma, a onda principal e as ondas secundárias, que ao excitar um número grande de ondas resulta em um espectro de banda larga.

Para descargas com bias positivo ou nulo, os espectros de potência da turbulência apresentam largura espectral grande e que aumenta quando o valor do bias aplicado aumenta. Além disso, essas descargas apresentam uma PDF com uma cauda acentuada, demonstrando que nestes disparos ocorrem frequêntes eventos extremos (bursts). Para essas descargas foi calculada a probabilidade de intervalo de tempo entre bursts e mostrado que essa probabilidade pode ser descrita por uma função exponencial.

Para investigar o transporte turbulento de partículas no Texas Helimak foram utilizadas técnicas de análise espectral (RITZ, 1988) para, a partir das medidas de oscilações eletrostáticas turbulentas, determinar o perfil radial do transporte de partículas como função do bias aplicado. Esses perfis foram então confrontados com a velocidade de fluxo do plasma e seu cisalhamento (shear). Esse exame revelou que os perfis radiais de transporte apresentam um ponto de mínimo nos casos em que o perfil radial do cisalhamento da velocidade tem um valor nulo (shearless). Utilizando um 
modelo Hamiltoniano (HORTON, 1981; MARCUS, 2008) de ondas de deriva, o ponto de mínimo no transporte de partículas foi interpretado como sendo resultado de uma barreira sem cisalhamento (barreira shearless).

Nos casos em que o perfil da velocidade não apresenta um máximo na região radial estudada, o mesmo modelo Hamiltoniano foi utilizado para interpretar os máximos apresentados nos perfis radiais do transporte como sendo ligados a um efeito de ressonância, em que a velocidade de fase da onda de deriva é igual à velocidade do plasma, que aumenta o transporte radial de partículas (TOUFEN, 2012).

A organização desta tese é descrita a seguir.

No capítulo 2 é apresentado o Texas Helimak, suas características e sistemas de diagnósticos. É apresentada também uma análise sobre o confinamento do plasma e a estabilidade MHD nessa máquina.

No capítulo 3 são apresentados e discutidos os principais métodos de análise utilizados neste trabalho.

No capítulo 4 são apresentadas as características do plasma não perturbado pelo bias externo, ou seja, os dados analisados neste capítulo foram adquiridos com bias nulo. Esse capítulo foi dividido de modo a apresentar separadamente as características de equilíbrio (valores médios) e da turbulência (flutuações) do plasma.

No capítulo 5 são estudadas as variação do plasma provocadas pela imposição do bias externo e a consequente alteração do perfil radial do campo elétrico. Novamente esse capítulo foi dividido entre equilíbrio e turbulência. É ainda neste capítulo que é discutida a região radial mais adequada para a análise da turbulência e do transporte no Texas Helimak.

No capítulo 6 são apresentadas as investigações e interpretações acerca das alterações da turbulência através do bias externo no Texas Helimak.

No capítulo 7 são apresentadas as investigações e interpretações acerca do transporte turbulento de partículas e seu controle através do bias externo no Texas Helimak.

Por fim, no capítulo 8 são apresentadas as conclusões e perspectivas deste trabalho. 


\section{TEXAS HELIMAK}

Neste capítulo serão apresentadas as características principais do Texas Helimak, máquina cujas experiências foram analisadas nesta tese.

O Texas Helimak pertence à classe de máquinas de plasma magneticamente confinado que se diferenciam dos tokamaks por apresentarem configurações de linhas de campo magnético mais flexíveis. Tais máquinas têm sido utilizadas no estudo de física básica de plasma e de métodos de diagnóstico. Esta classe de máquinas inclui também as máquinas cilíndricas como o LAPD (GEKELMKAN, 1991; HORTON, 2005). Os stellerators como o TJ-II (HIDALGO, 2005) e o LHD (MOTOJIMA, 1999), apesar de sua flexibilidade são, historicamente, considerados como máquinas de fusão. Atualmente, pode-se destacar três máquinas do tipo Helimak: o TORPEX (FASOLI, 2006), o BLAAMANN (RYPDAL, 2004) e o Texas Helimak (GENTLE, 2008).

A configuração helimak, também chamada de SMT (simples tórus magnetizado), permite a existência de equilíbrio magnetohidrodinâmico (MHD) e mostra-se como uma ferramenta promissora para o estudo de plasmas magneticamente confinados (RYPDAL, 2005). Um helimak tem como característica principal um campo magnético helicoidal, composto por um campo toroidal, $B_{\phi}$, somado a um campo vertical, $B_{Z}$, menos intenso (ZIMMERMAN, 1993). Dessa forma suas linhas de campo magnético circulam em torno do eixo principal formando helicóides. A Figura 2.1 mostra, em azul, um exemplo das linhas de campo magnético helicoidais no Texas Helimak.

O Helimak, além de ser um experimento interessante sobre física de plasmas também apresenta semelhança com determinadas regiões do plasma em tokamaks. Por apresentar um plasma com baixa densidade e temperatura, pode-se dizer que o plasma em um helimak se assemelha com as regiões da borda (plasma edge) e externa (scrapeoff-layer) do plasma de um tokamak (LI, 2009). Máquinas do tipo helimak têm atraído interesse devido a esta semelhança, como pode ser visto em RICCI (2008) e em MÜLLER (2004), mas também pela simplicidade de sua representação analítica (RICCI, 2009). Outra vantagem do helimak é que, devido à baixa densidade e temperatura do seu plasma é possível implantar sondas em qualquer região do plasma. Um exemplo é a sonda de imagens hexagonais da turbulência - HEXTIP (MÜLLER, 2005) implantado no TORPEX. 


\subsection{Características do Texas Helimak}

O Texas Helimak esta localizado no Institute for Fusion Studies, na Universidade do Texas em Austin. Seu vaso de aço inoxidável foi desenvolvido para o tokamak TEXT (GENTLE, 1984) e possui seção reta retangular com dois metros de altura, raio menor de $0,6 \mathrm{~m}$ e raio maior de $1,6 \mathrm{~m}$. No topo e no fundo do vaso estão fixados quatro conjuntos de quatro placas condutoras, dezesseis placas no total, chamadas de "placas de bias". Cada conjunto de placas está separado em $180^{\circ}$ do conjunto oposto, como mostrado na Figura 2.1. As linhas de campo magnético do Helimak circulam em torno do eixo principal e começam e terminam nas placas de bias, de modo que é através delas e do próprio vaso que as linhas de campo se fecham. Estas placas, além de fechar as linhas de campo ainda possuem duas outras funções: são utilizadas como suporte para a colocação de sondas de Langmuir e para a imposição de potencial elétrico externo ao plasma (bias). Assim, o vaso e as placas são mantidas aterradas, a menos do conjunto utilizado para impor o bias que pode ser conectado a uma fonte de tensão externa. As placas utilizadas para a imposição de bias em todos os dados analisados neste trabalho estão destacadas em amarelo na Figura 2.1.

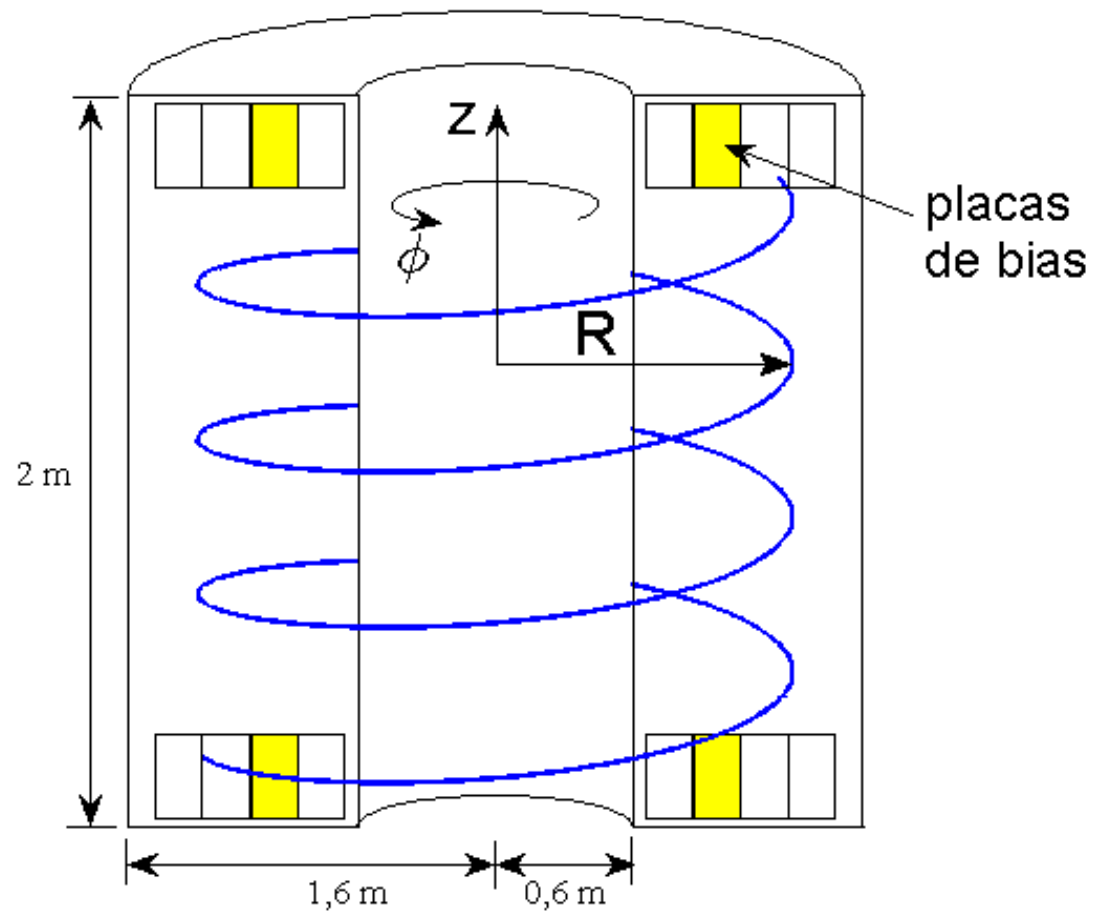

Figura 2.1: Desenho esquemático do Texas Helimak mostrando um exemplo de linha de campo magnético em azul e os quatro conjuntos de placas de bias no topo e fundo da máquina. As placas utilizadas para a aplicação de potencial externo (bias) nas experiências analisadas estão representadas em amarelo. 
O campo magnético toroidal $B_{\emptyset}$ do Texas Helimak é da ordem de 0,1 T. Este é produzido por um conjunto de dezesseis bobinas, vistas em vermelho na Figura 2.2, com 28 voltas cada. O campo vertical $B_{z}$, que pode atingir até $10 \%$ do valor de $B_{\varnothing}$ (PEREZ, 2006), é produzido por três bobinas, vistas em azul na Figura 2.2, com 126 voltas nas bobinas de topo e fundo e 75 voltas na bobina do meio da máquina. As bobinas são alimentadas por um sistema de doze retificadores controlados de silício (SCR) capaz de fornecer 500V a 1500A.

O campo magnético vertical pode ser ajustado adicionando-se resistências em série com as bobinas verticais. Esse ajuste provoca a alteração do comprimento de conexão (conection length) que é definido como o comprimento total da linha de campo magnético desde o fundo até o topo do vaso. No Texas Helimak o comprimento de conexão no centro radial do vaso $\left(R_{0}=1,10 \mathrm{~m}\right)$ pode ser ajustado de dez metros até alguns quilômetros (GENTLE, 2008).

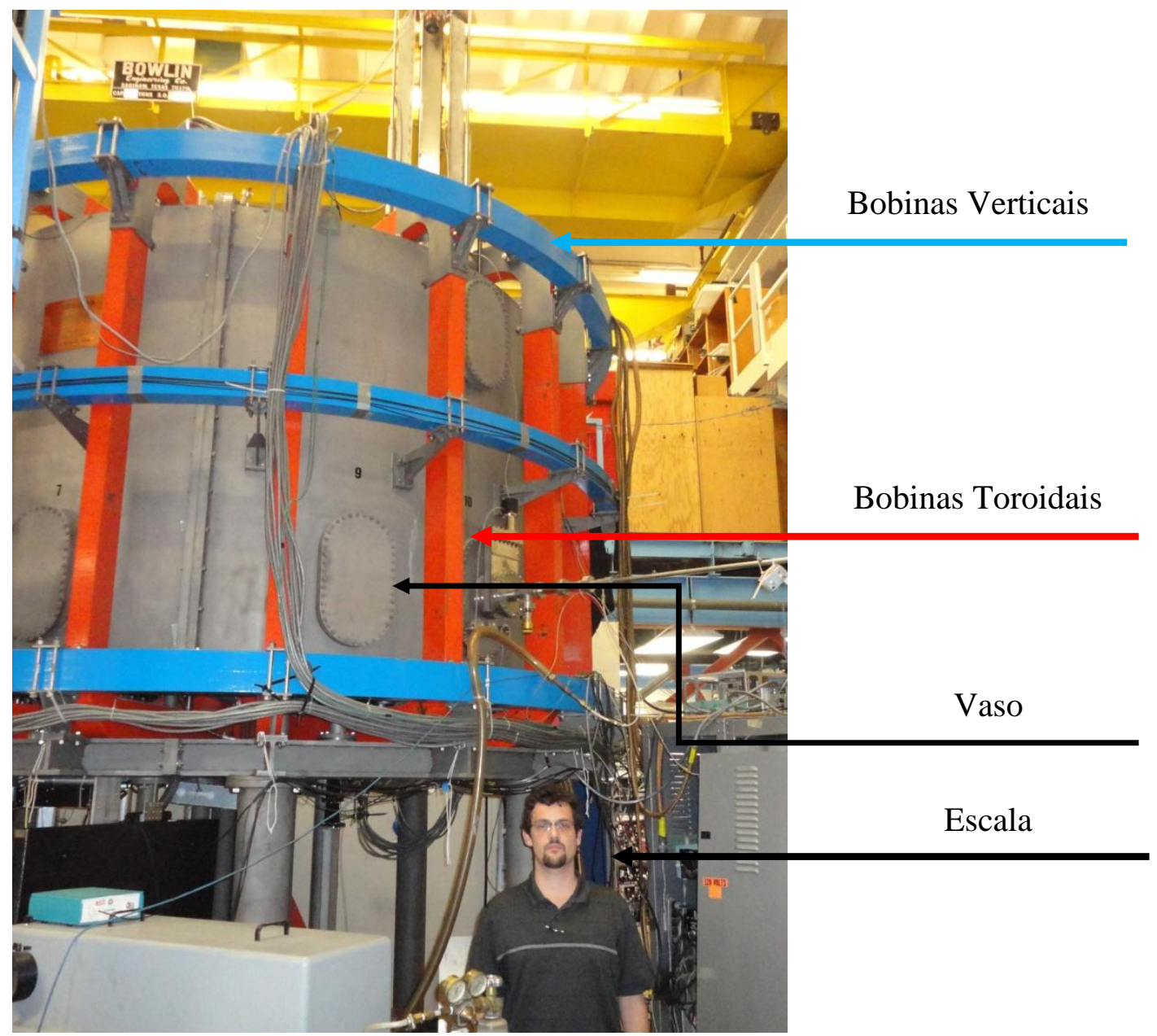

Figura 2.2: Foto do Texas Helimak mostrando o vaso de aço inox, os conjuntos de bobinas toroidais em vermelho e das bobinas verticais em azul. 
No Texas Helimak o plasma é aquecido através de uma onda do tipo ECRH (electron cyclotron resonance heating) (CAIRNS, 1991) com frequência fundamental de 2,45 GHz e potência de $6 \mathrm{~kW}$. Essas microondas são conduzidas da fonte, através de guias de onda, até uma janela de quartzo no lado interno do vaso. O Helimak pode operar com os gases nobres: hélio, neônio, xenônio e argônio, sendo este último o utilizado em todas as experiências analisadas neste trabalho. O vácuo no Texas Helimak é obtido através de um bomba turbo-molecular com capacidade de $1 \mathrm{~m}^{3} / \mathrm{s}$.

Na Tabela 2.1 observam-se alguns dos parâmetros relevantes para o Texas Helimak. Para o cálculo destes parâmetros admitiu-se que o campo magnético médio é $0,07 \mathrm{~T}$, a densidade média é $2 \times 10^{16} \mathrm{~m}^{-3}$, a temperatura eletrônica média é $12 \mathrm{eV}$, a temperatura média dos íons é $0,03 \mathrm{eV}$ e que o campo elétrico radial médio vale $5 \mathrm{~V} / \mathrm{m}$.

Tabela 2.1: Parâmetros relevantes para o Texas Helimak.

\begin{tabular}{|lr|}
\hline frequência giroscópica do elétron $(\mathrm{Hz})$ & $f_{c e}=2,0 \times 10^{9}$ \\
frequência giroscópica do íon $(\mathrm{Hz})$ & $f_{c i}=2,7 \times 10^{4}$ \\
frequência de plasma do elétron $(\mathrm{Hz})$ & $f_{p e}=1,3 \times 10^{9}$ \\
frequência de plasma do íon $(\mathrm{Hz})$ & $f_{p i}=4,7 \times 10^{6}$ \\
taxa de colisão dos elétrons $\left(\mathrm{s}^{-1}\right)$ & $v_{e e}=5,5 \times 10^{3}$ \\
taxa de colisão dos íons $\left(\mathrm{s}^{-1}\right)$ & $v_{i i}=1,5 \times 10^{5}$ \\
comprimento de debye $(\mathrm{m})$ & $\lambda_{D}=1,8 \times 10^{-4}$ \\
raio de giro dos elétrons $(\mathrm{m})$ & $\rho_{e}=1,7 \times 10^{-4}$ \\
raio de giro dos íons $(\mathrm{m})$ & $\rho_{i}=2,3 \times 10^{-3}$ \\
raio de giro acústico dos íons $(\mathrm{m})$ & $\rho_{s}=4,5 \times 10^{-2}$ \\
velocidade térmica dos elétrons $(\mathrm{m} / \mathrm{s})$ & $\mathrm{v}_{T e}=2,1 \times 10^{6}$ \\
velocidade térmica dos íons $(\mathrm{m} / \mathrm{s})$ & $\mathrm{v}_{T i}=3,9 \times 10^{2}$ \\
velocidade do som dos íons $(\mathrm{m} / \mathrm{s})$ & $c_{s}=5,4 \times 10^{3}$ \\
velocidade de Alfvén $(\mathrm{m} / \mathrm{s})$ & $\mathrm{v}_{A}=1,7 \times 10^{6}$ \\
velocidade de deriva elétrica $(\mathrm{m} / \mathrm{s})$ & $\mathrm{v}_{E}=7,1 \times 10^{1}$ \\
\hline
\end{tabular}

A seguir, na tabela 2.2, é apresentada uma comparação dos valores típicos de densidade e temperatura no Texas Helimak e no tokamak TCABR (do IF-USP). 
Tabela 2.2: Comparação de parâmetros típicos do Tokamak TCABR com o Texas Helimak, mostrando que os parâmetros típicos do Texas Helimak são semelhantes aos da região da borda e SOL do TCABR. ( GALVÃO, 2001; DOS SANTOS LIMA, 20091 e GENTLE, 2008).

\begin{tabular}{|c|c|c|c|}
\hline \multicolumn{2}{|c|}{ Parâmetros do TCABR: } & \multicolumn{2}{c|}{ Parâmetros do Texas Helimak: } \\
\hline Raio maior: & $R_{\text {maior }}=0,61 \mathrm{~m}$ & Raio maior: & $R_{\text {maior }}=1,6 \mathrm{~m}$ \\
\hline Raio menor: & $R_{\text {menor }}=0,18 \mathrm{~m}$ & Raio menor: & $R_{\text {menor }}=0,6 \mathrm{~m}$ \\
\hline $\begin{array}{c}\text { Densidade central } \\
\text { de elétrons: }\end{array}$ & $\begin{array}{c}n_{e}=1 \mathrm{a} 4 \times 10^{19} \\
\mathrm{~m}^{-3}\end{array}$ & \multirow{2}{*}{$\begin{array}{c}\text { Densidade dos } \\
\text { elétrons: }\end{array}$} & $n_{e} \leq 10^{17} \mathrm{~m}^{-3}$ \\
\cline { 1 - 2 } $\begin{array}{c}\text { Densidade de } \\
\text { elétrons no SOL : }\end{array}$ & $n_{e}=1,5 \times 10^{18} \mathrm{~m}^{-3}$ & $\begin{array}{c}\text { Temperatura típica } \\
\text { dos elétrons }\end{array}$ & $T_{e_{-} \text {média }}=10 \mathrm{eV}$ \\
\cline { 1 - 2 } $\begin{array}{c}\text { Temperatura média } \\
\text { de elétrons : }\end{array}$ & $T_{e_{-} \text {média }}=400 \mathrm{eV}$ & \\
\cline { 1 - 2 } $\begin{array}{c}\text { Temperatura dos } \\
\text { elétrons na borda: }\end{array}$ & $T_{e}=10 \mathrm{a} 20 \mathrm{eV}$ & \\
\hline $\begin{array}{c}\text { Temperatura dos } \\
\text { elétrons no SOL: }\end{array}$ & $T_{e_{-} S O L}=5 \mathrm{eV}$ & & \\
\hline
\end{tabular}

Ao observar a tabela 2.2, nota-se que o plasma existente em uma grande faixa radial do Texas Helimak tem características de temperatura e densidade semelhantes às das regiões de borda e SOL de um tokamak, assim as experiências de controle no Texas Helimak podem fornecer subsídios para o controle do transporte de partículas na borda de tokamaks.

\subsection{Sistemas de diagnóstico}

O Texas Helimak conta com um grande conjunto de sondas de Langmuir (MOTT-SMITH, 1926) como principal sistema de diagnóstico do plasma. Ao todo são aproximadamente 700 sondas montadas nos quatro conjuntos de placas de bias, vide Figura 2.1, de modo que as sondas estão distribuídas ao longo da direção radial do vaso e cobrem uma faixa de mais de $10 \mathrm{~cm}$ de altura, tanto no fundo quanto no topo da máquina. A Figura 2.3 mostra um exemplo de uma das placas de bias e seu conjunto de sondas de Langmuir enquanto a Figura 2.4 mostra a posição de um dos conjuntos de quatro placas dentro do vaso do Texas Helimak. 


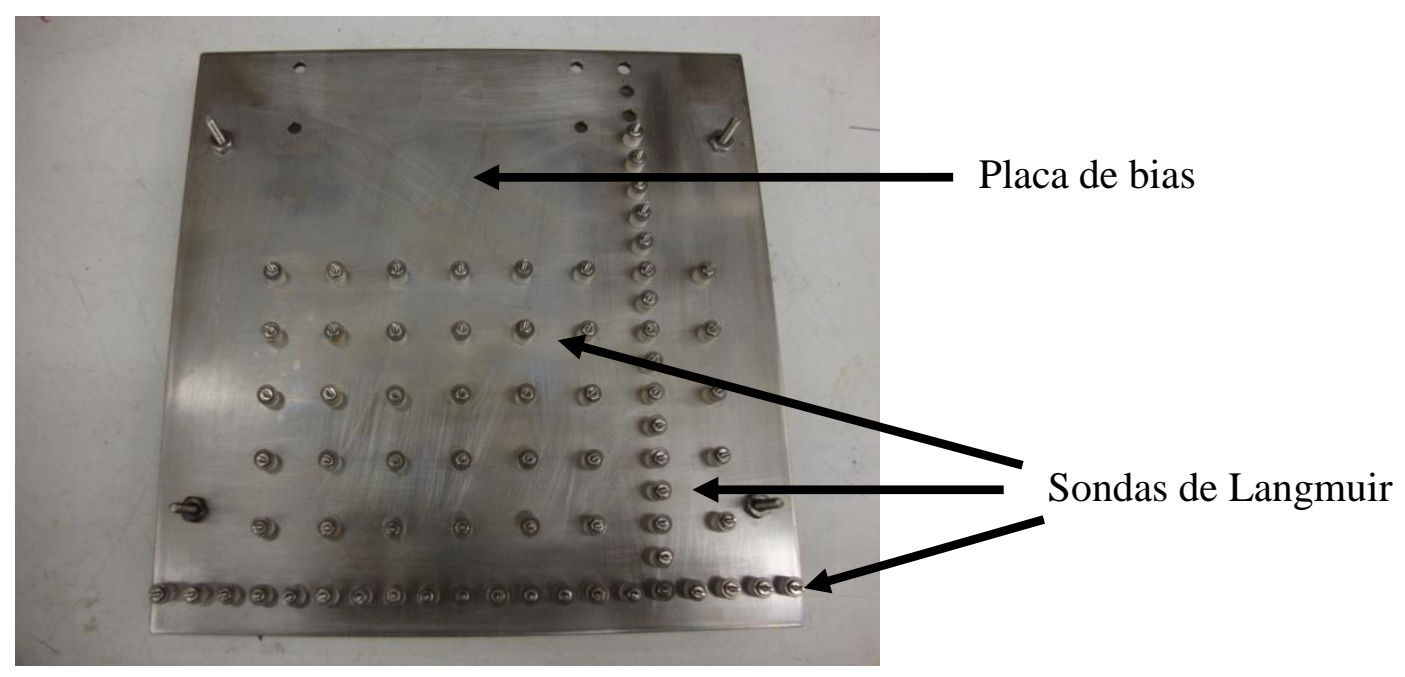

Figura 2.3: Foto de uma das placas de bias, indicadas na Figura 2.1, mostrando as sondas de Langmuir distribuídas na superfície da placa.
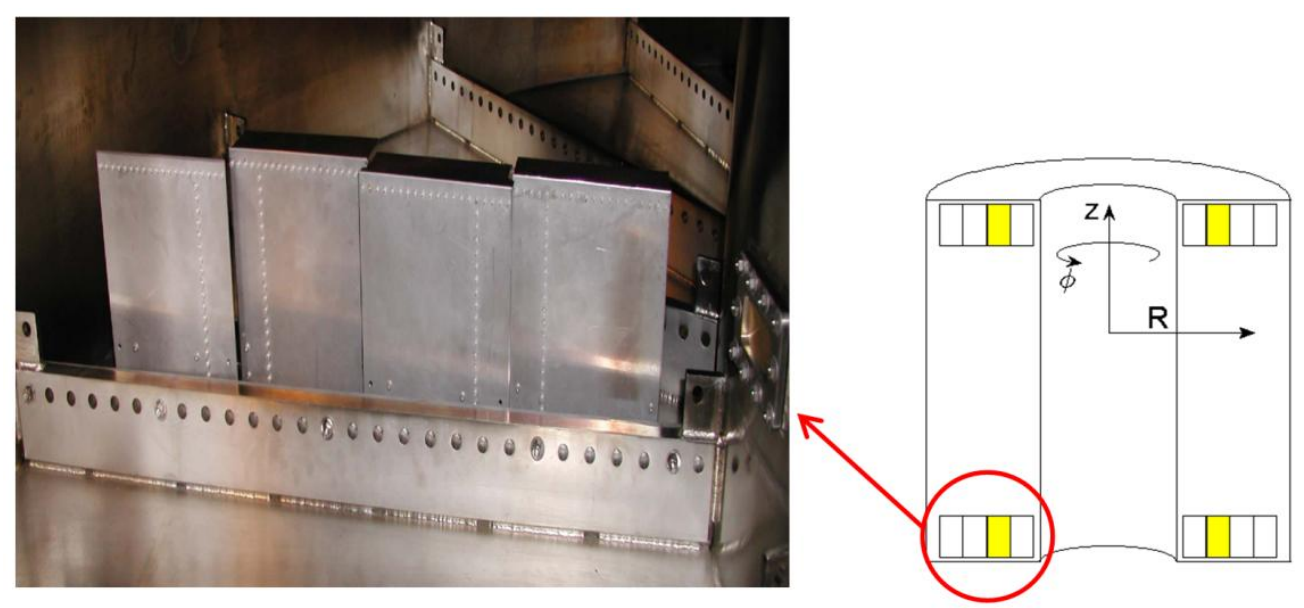

Figura 2.4: Foto de um dos quatro conjuntos de quatro placas de bias, indicadas na Figura 2.1, montadas no fundo do vaso do Texas Helimak. (fonte: GENTLE, K. W.; disponível em < http://www.ph.utexas.edu/ phy315>) .

Para a aquisição do sinal proveniente destas sondas são utilizados três conjuntos de conversores analógico digital (ADC): um com 128 canais e $7 \mathrm{kHz}$ de taxa de amostragem, outro com 16 canais e $100 \mathrm{kHz}$ e um último, implantado em 2011, com 96 canais e $500 \mathrm{kHz}$ de taxa de amostragem. O ADC mais lento é utilizado para monitoramento de parâmetros do equipamento e medida de perfis médios enquanto os ADCs mais rápidos são utilizados para medidas de flutuações. Os dados das sondas são armazenados em um sistema MDSplus (STILLERMAN, 1997). A Figura 2.5 mostra um exemplo de sinais de corrente de saturação iônica medidos ao longo de todo o disparo em quatro posições radiais distintas e mesma posição vertical. 

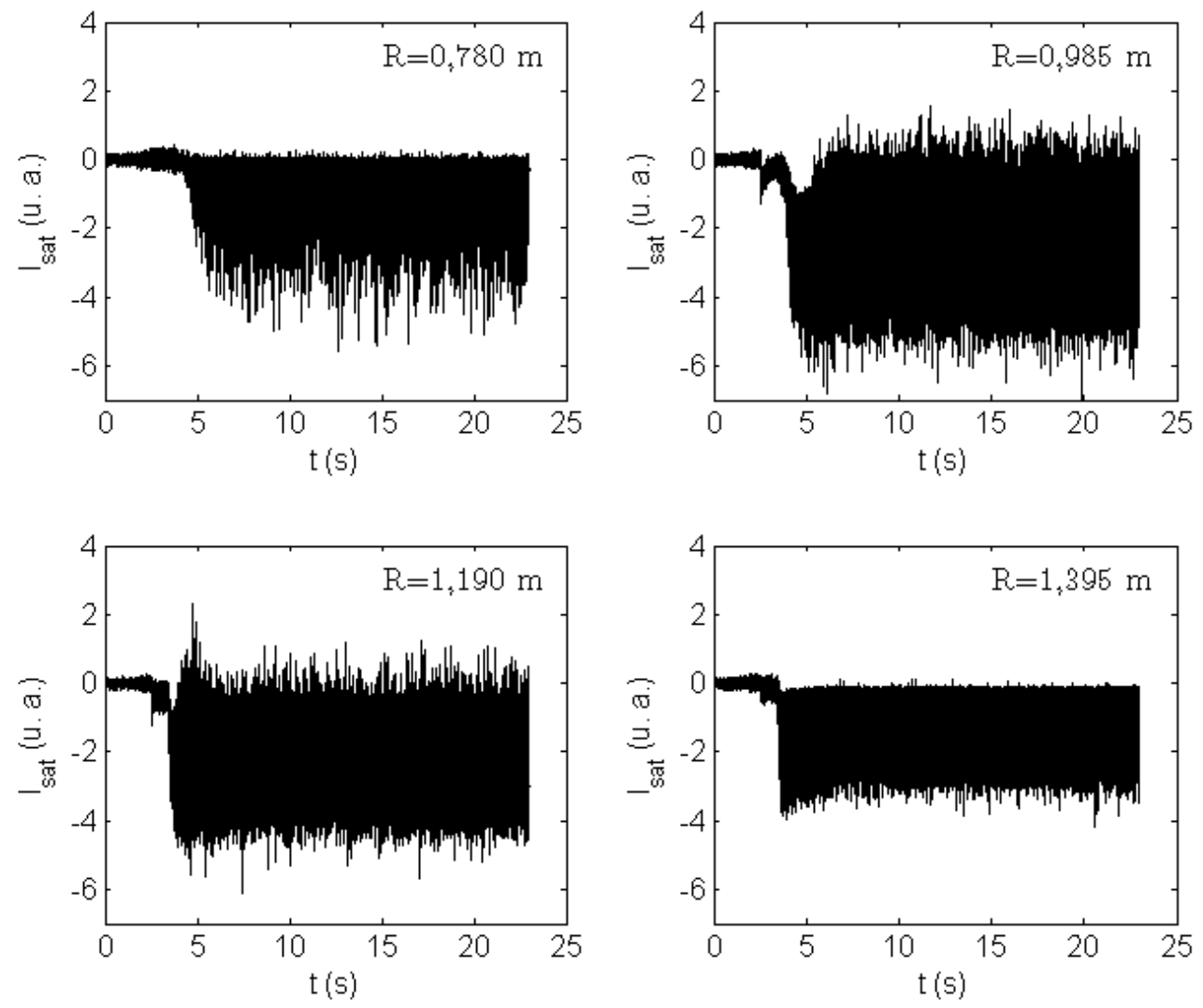

Figura 2.5: Exemplo de quatros medidas de corrente de saturação iônica no mesmo disparo utilizando quatro sondas posicionadas em quatro raios diferentes e na mesma posição vertical $(\mathrm{z}=1,747 \mathrm{~m})$.

Além do conjunto de sondas de Langmuir, o Texas Helimak possui um espectrômetro capaz de medir o perfil radial da velocidade vertical média (em z) de fluxo de plasma, $V_{Z}(R)$, numa posição radial, através do deslocamento Doppler das linhas de emissão do ion, no caso argônio (Spectroscopic flow measurement). A técnica utilizada no Texas Helimak é semelhante à desenvolvida para medidas de espectroscopia no Alcator $C$-mod Tokamak (ROWAN, 2008 e BESPAMYATNOV, 2010). Esta técnica pode ser aplicada com maior simplicidade no Texas Helimak, uma vez que o fluxo do plasma deve ser principalmente na direção vertical, e não poloidal como em um tokamak, o que permite posicionar o espectrômetro (abaixo do vaso) diretamente na direção do fluxo para a medida do deslocamento Doppler. O arranjo experimental utilizado para a captação do espectro do plasma, que é montado na parte de baixo do tanque do Helimak, é visto na Figura 2.6 e o espectrômetro utilizado nestas medidas é visto na Figura 2.7. 


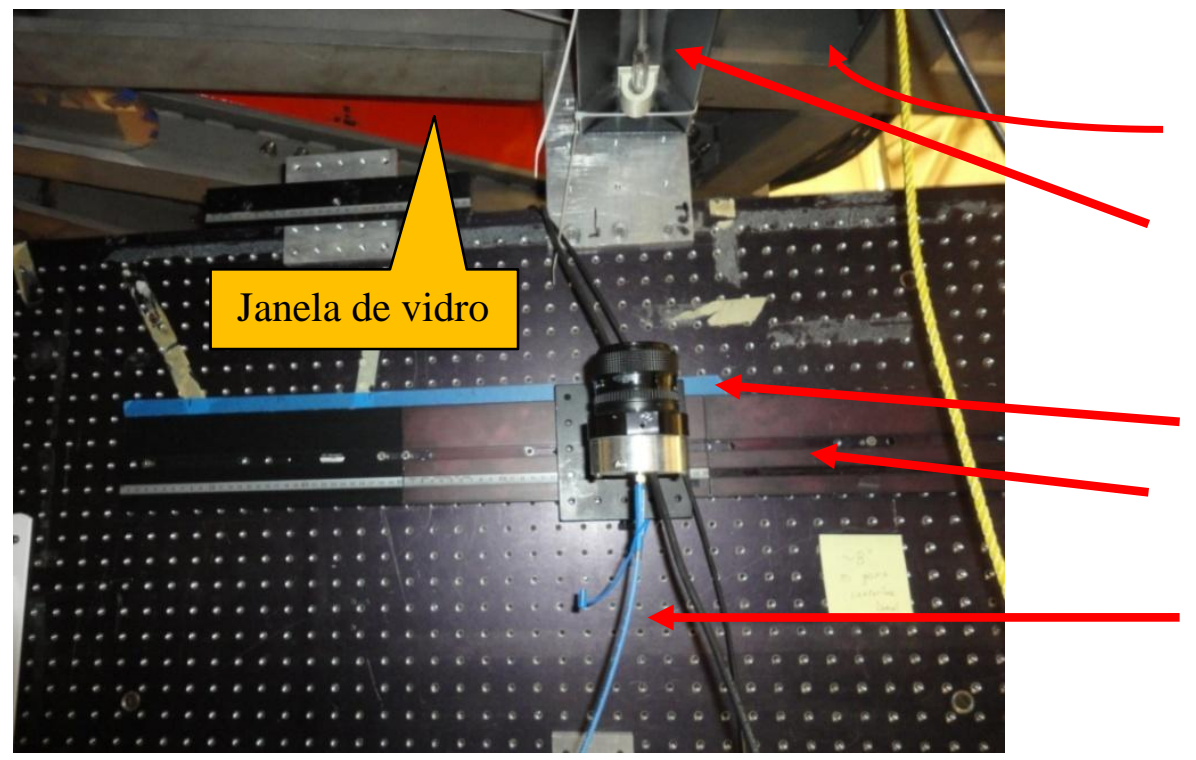

Helimak

Lâmpada de hidrogênio de referência

Lente de zoom.

Trilho de montagem

Fibra óptica

Figura 2.6: Arranjo experimental utilizado para medidas espectroscópicas de velocidade de fluxo do plasma $\left(V_{z}\right)$.

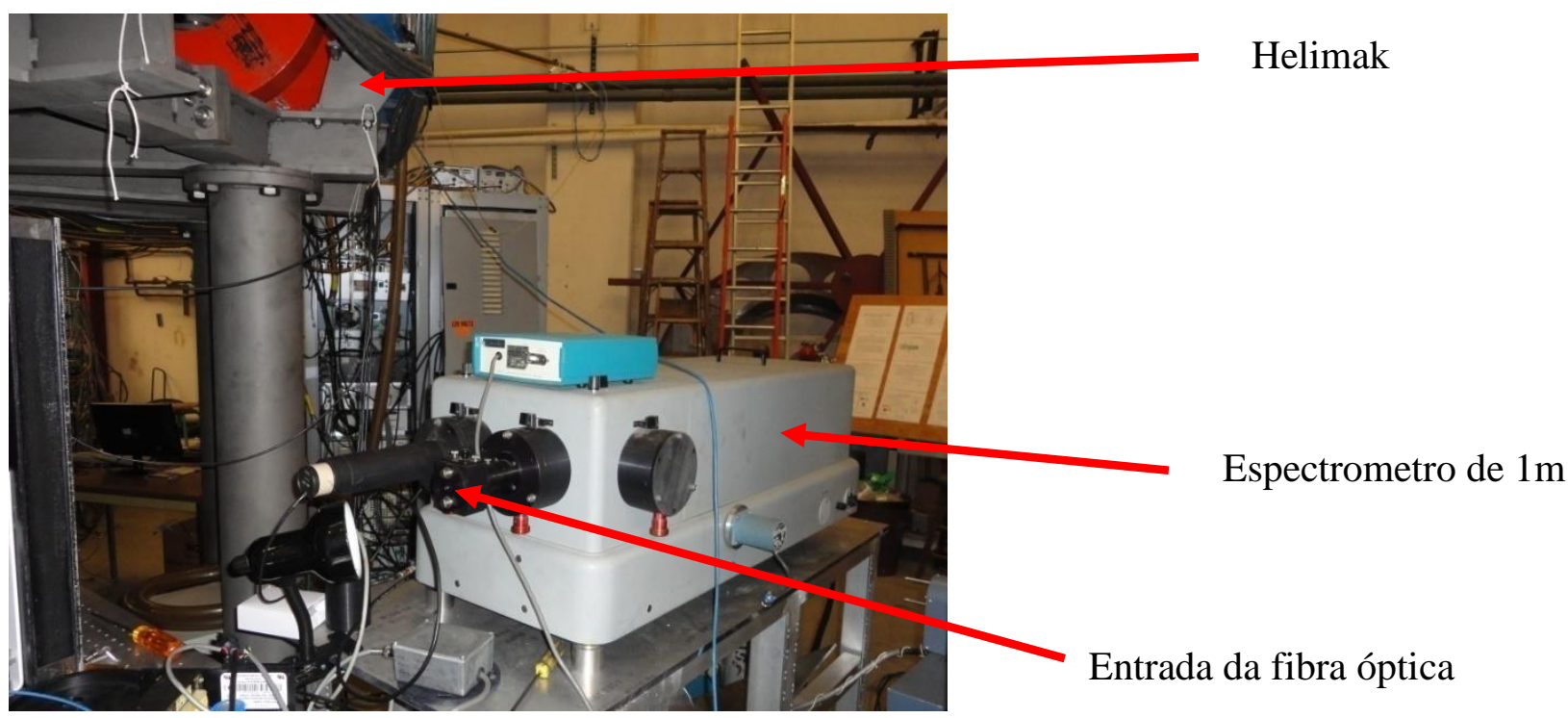

Figura 2.7: Espectrômetro utilizado para medidas de velocidade de fluxo do plasma $\left(V_{z}\right)$.

\subsection{Existência de equilíbrio e estabilidade MHD no Texas Helimak}

Esta seção tem por objetivo introduzir brevemente o equilíbrio MHD na configuração helimak, mostrando a importância da componente vertical do campo magnético, e fornecer um critério de estabilidade MHD do plasma baseado no critério de Suydam (TAYLER, 1961). 


\subsubsection{Equilíbrio MHD}

Na configuração magnética do helimak, a velocidade de deriva devida ao gradiente e à curvatura do campo magnético, será vertical e dependente da carga. A soma destas duas velocidades de deriva pode ser escrita como:

$\mathbf{V}_{R}+\mathbf{V}_{\nabla \mathbf{B}} \propto \frac{\mathbf{R}_{c} \times \mathbf{B}}{q} \Rightarrow \mathbf{V}_{R}+\mathbf{V}_{\nabla \mathbf{B}} \propto-\mathbf{e}_{z} \frac{1}{q}$

Sendo $\mathbf{R}_{c}=R_{c}\left(-\mathbf{e}_{r}\right), R_{c}$ o raio de curvatura, $\mathbf{B}=B\left(\mathbf{e}_{\varphi}\right)$ a componente toroidal do campo magnético e $q$ a carga do elétron ou do íon.

Essa velocidade irá produzir uma separação vertical das cargas elétricas e consequentemente um campo elétrico na direção $z$. Este campo elétrico vertical, por sua vez, irá produzir uma velocidade de deriva elétrica:

$\mathbf{V}_{E}=\frac{\mathbf{E} \times \mathbf{B}}{\mathbf{B}^{2}}$

A componente radial da velocidade de deriva elétrica age empurrando o plasma contra a parede e, consequentemente, destruindo o confinamento. Para evitar que isso ocorra a separação vertical de cargas provocada pela velocidade de deriva $\mathbf{v}_{R}+\mathbf{v}_{\nabla \boldsymbol{B}}$ deve ser anulada. Para isso é preciso que exista um "caminho" vertical para que as cargas possam se delocar verticalmente. É a componente vertical do campo magnético, que faz com que as linhas do campo magnético sejam helicoidais, permitindo um deslocamento vertical das cargas. Assim sendo, as cargas elétricas, separadas pela velocidade de deriva $\mathbf{v}_{R}+\mathbf{v}_{\nabla B}$, vão se anular ao se deslocar na direção vertical por seguirem as linhas helicoidais. Esse deslocamento de cargas na direção vertical é necessário para cancelar a polarização causada pela velocidade de deriva $\mathbf{v}_{R}+\mathbf{v}_{\nabla \boldsymbol{B}}$. Concluindo, para que exista equilíbrio MHD no helimak, é necessário que as linhas de campo magnético do helimak sejam helicoidais.

Deste que possua uma componente vertical do campo magnético não nulo, o plasma em um helimak pode satisfazer, aproximadamente, a condição de equilíbrio MHD, com os perfis radiais da pressão, campo magnético e corrente elétrica relacionados pela equação de Grad-Shafranov (BITTENCOURT, 1986). Como 
demonstrado por LUCKHARDT (1999), existe solução analítica da equação de GradShafranov, para o equilíbrio aplicável ao helimak, devido a sua simetria axial simplificada.

\subsubsection{Estabilidade MHD}

Em primeira aproximação, pode-se considerar um plasma uniforme no interior do helimak, exceto na direção radial. Assim sendo:

$$
\begin{aligned}
& p=p(r), \\
& \rho=\rho(r), \\
& \mathbf{V}=V_{\varphi}(r) \mathbf{e}_{\varphi}+V_{z}(r) \mathbf{e}_{z}, \\
& \mathbf{B}=B_{\varphi}(r) \mathbf{e}_{\varphi}+B_{z}(r) \mathbf{e}_{z} .
\end{aligned}
$$

Sendo $p$ a pressão no plasma, $\rho$ a densidade, $\mathbf{V}$ a velocidade das partículas, $\mathbf{B}$ o campo magnético e $\varphi$ a coordenada toroidal.

A equação de balanço de força poderá então ser escrita como (LUCKHARDT, 1999):

$$
\frac{d}{d r}\left(p+\frac{B_{z}^{2}}{2 \mu_{o}}\right)+\frac{B_{\varphi}}{\mu_{o} r} \frac{d}{d r}\left(r B_{\varphi}\right)-\frac{\rho v_{\varphi}^{2}}{r}=0
$$

Definindo o fator de segurança $q(r)$ para o helimak,

$$
q(r)=\frac{H B_{\varphi}}{\pi B_{z} r},
$$

sendo $H$ a metade da altura total do helimak, e assumindo uma perturbação magnética da forma:

$$
\xi(r, \varphi, z)=\xi(r) e^{\left(i n \varphi+i k_{z} z\right)}
$$

cujo vetor de onda, $\mathbf{k}$, é dado por

$$
\mathbf{k}=\frac{n}{r} \hat{\mathbf{e}}_{\varphi}+k_{z} \hat{\mathbf{e}}_{z},
$$


tem-se que a estabilidade MHD linear é obtida pelo critério de Suydam (TAYLER, 1961), para um campo magnético não uniforme com um cisalhamento magnético descrito por $q^{\prime}$, o gradiente radial de $q$ (PEREZ, 2006). Assim, a condição necessária e suficiente para a estabilidade do plasma é:

$$
\frac{2 \mu_{o} p^{\prime}(r)}{B_{z}^{2}(r)}+\left.\frac{r}{4}\left[\frac{q^{\prime}(r)}{q(r)}\right]^{2}\right|_{r=r_{s}}>0 .
$$

O raio $r_{s}$ marca a posição radial das superfícies de ressonância criadas pela perturbação magnética assumida:

$$
r_{s}=\frac{n}{l} \frac{B_{z} \pi}{H B_{\varphi}} \quad \begin{aligned}
& n=1,2,3, \ldots \\
& l=1,2,3, \ldots
\end{aligned} .
$$

Nestas superfícies de ressonância, o campo magnético é perpendicular ao vetor de onda.

Ao observar a Equação 2.11, pode-se perceber que, tomada uma região em torno de $r_{s}$, a estabilidade é esperada até um limite superior para o módulo do gradiente radial da pressão, $p^{\prime}(r)$.

Como demonstrado por LUCKHARDT (1999), existe solução analítica da equação de Grad-Shafranov, na aproximação MHD (BITTENCOURT, 1986), para o equilíbrio aplicável ao helimak, devido a sua simetria axial simplificada. Utilizando essa solução e analisando o critério de estabilidade mostrado acima para os limites de baixo e alto cisalhamento (shear), o autor encontra uma condição necessária e suficiente para que exista equilíbrio estável para modos MHD locais:

$$
\frac{2 \mu_{0}\langle p\rangle}{B_{Z}^{2}}<-\frac{1}{\frac{1}{p} \frac{d p}{d r} R_{M}},
$$

onde $R_{M}$ é o valor do raio maior do helimak.

A equação 2.13 fornece um critério mais geral do que o dado pela equação 2.11, porém ela depende da solução da equação de Grad-Shafranov utilizada por LUCKHARDT (1999).

Para verificar a relação da equação 2.13 no intervalo radial analisado neste trabalho, $1,10 \mathrm{~m}<\mathrm{R}<1,30 \mathrm{~m}$, é preciso estimar o valor médio da pressão e seu gradiente radial. Para isso assumiu-se que: 


$$
\begin{aligned}
& \langle p\rangle \cong k T e\langle n\rangle V^{-1}, \\
& \frac{1}{p} \frac{d p}{d r} \cong \frac{1}{n} \frac{d n}{d R},
\end{aligned}
$$

com a temperatura do plasma aproximadamente uniforme. Com isso o valor médio da pressão e seu gradiente radial foram estimados utilizando o perfil radial médio de densidade volumétrica de partículas medido em disparos com bias nulo, mostrado na Figura 2.8. Além disso, adotando-se: $k T_{e}=13 \mathrm{eV}, B_{z}=1,4 \times 10^{-3} \mathrm{~T}$ e $R_{M}=1,6 \mathrm{~m}$, a equação 2.13 foi utilizada para confirmar a estabilidade MHD no Texas Helimak:

$$
\frac{2 \mu_{0}\langle p\rangle}{B_{Z}^{2}}<-\frac{1}{\frac{1}{p} \frac{d p}{d r} R_{M}} \Rightarrow 0,04<0,22 .
$$

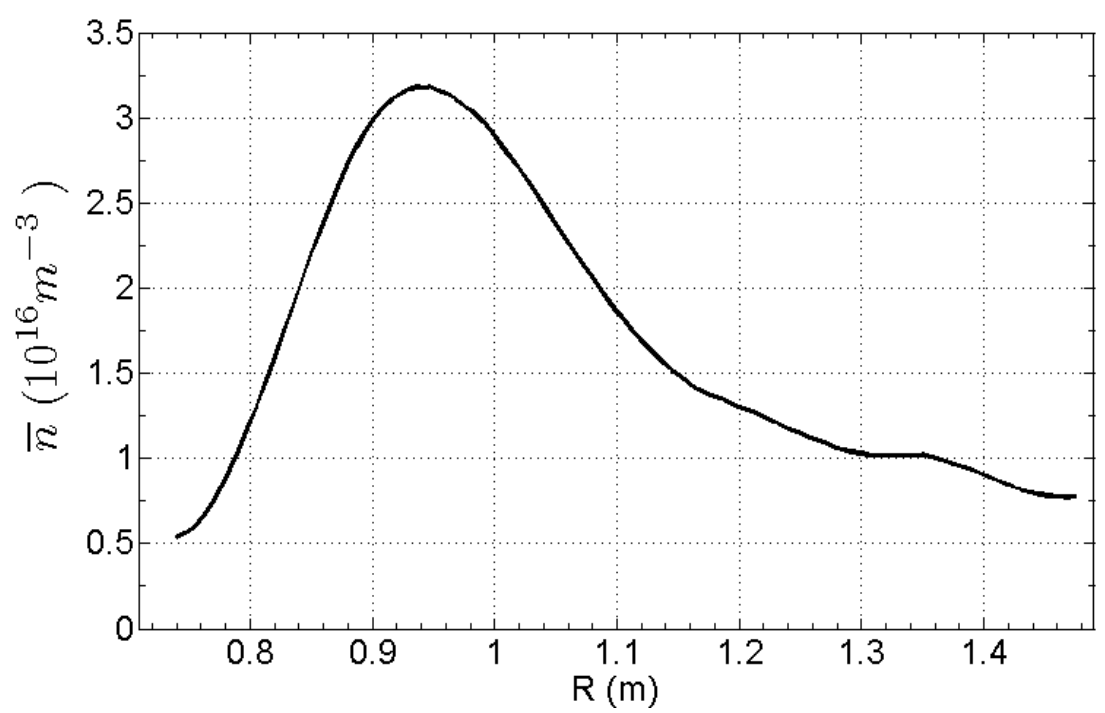

Figura 2.8: Perfil radial experimental de densidade volumétrica de partículas do Texas Helimak medido em $\mathrm{z}=1,747$ e em disparos com bias nulo.

Experimentalmente, até o presente momento, não foram observados indícios de que o plasma na configuração helimak apresente instabilidades magnéticas importantes (PEREZ, 2006 e FASOLI, 2006), o que indica que os limites de estabilidade definidos pela equação 2.13 podem ser facilmente satisfeitos.

Por outro lado, devido ao gradiente radial de densidade, à curvatura do campo magnético e ao seu cisalhamento, é esperada a existência de turbulência eletrostática. Essa turbulência seria iniciada por instabilidades eletrostáticas com modos característicos. Como já investigado no TORPEX (POLI, 2007) e no Texas Helimak, 
essas instabilidades são do tipo Raleigh-Taylor (interchange instability) (LI, 2011), Kelvin-Helmholtz, e de ondas de deriva (drift wave instabilities) (PEREZ, 2006). Como mostrado por POLI (2007), também são observadas instabilidades caracterizadas por uma mistura desses modos característicos (drift-intercharge instabilities).

\subsection{Imposição de potencial elétrico externo: bias}

Como mostrado por VAN OOST (2003), o uso de eletrodos para imposição de potencial elétrico e controle da turbulência do plasma e transporte de partículas têm sido amplamente utilizado.

Uma das características mais interessantes do Texas Helimak é sua capacidade de alterar o perfil radial do campo elétrico radial através da imposição de um potencial elétrico externo ao plasma, que neste trabalho será chamado de bias. A Figura 2.9 mostra um disparo em que o valor do bias se altera ao longo do tempo da descarga e ilustra como o bias pode ser utilizado para o controle e redução da turbulência. Nota-se nesta figura que, à medida que o valor de bias torna-se mais negativo (no intervalo: $8 \mathrm{~s}$ $<\mathrm{t}<12 \mathrm{~s}$ ), a amplitude das oscilações da corrente de saturação iônica se reduz. Observa-se também que quando o valor do bias volta a subir (para t $>13 \mathrm{~s}$ ) a amplitude da turbulência volta a crescer.

Embora o exemplo da Figura 2.9 apresente um disparo em que o valor de bias varia com o tempo, nos dados analisados neste trabalho o valor de bias foi mantido constante durante toda a descarga. Em todos os disparos analisados neste trabalho o bias, quando aplicado, foi imposto em quatro placas de bias com, a mesma posição radial (de $R=0,863 \mathrm{~m}$ até $\mathrm{R}=1,075 \mathrm{~m}$ ), vistas em amarelo na Figura 2.1. 

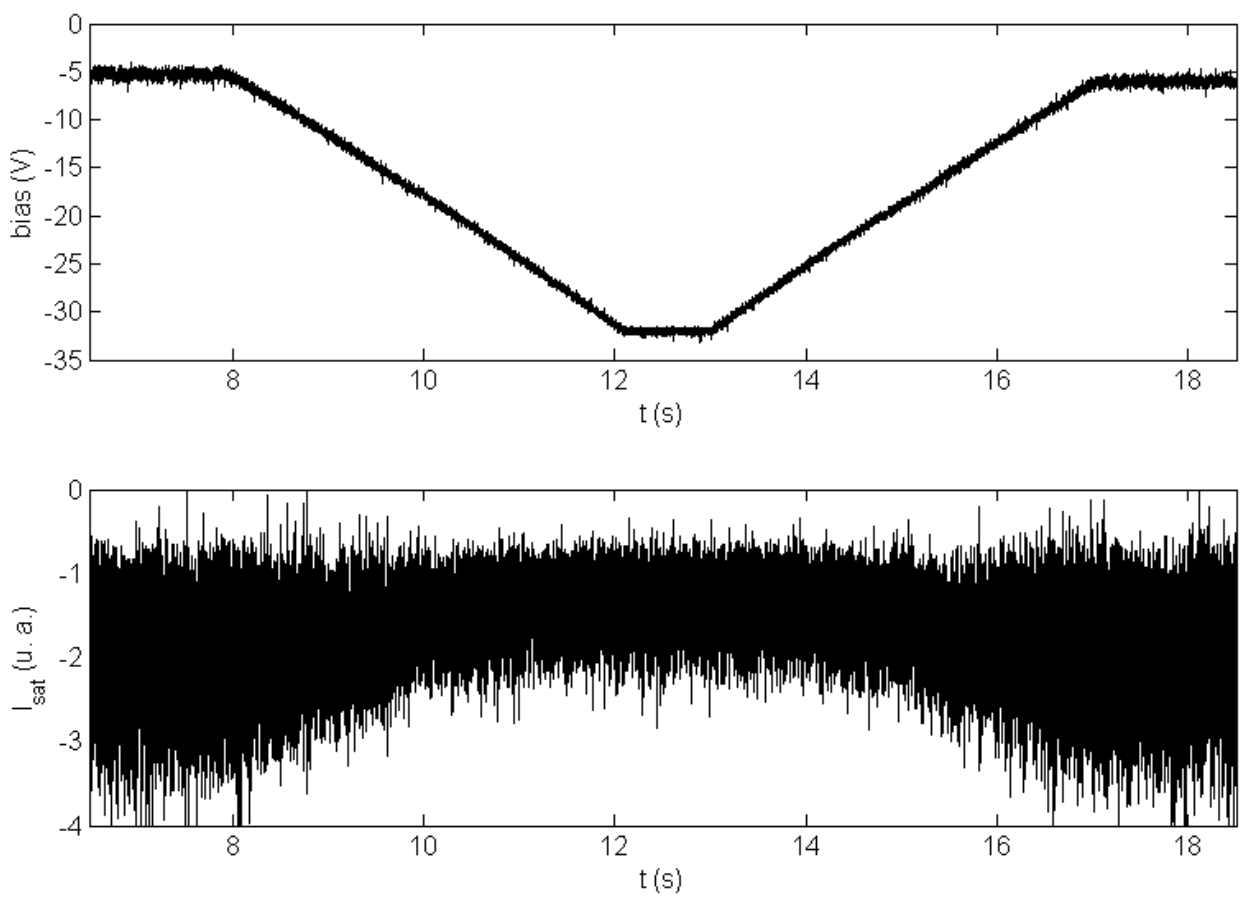

Figura 2.9: Evolução temporal do bias e da corrente de saturação iônica em um disparo em que o bias muda ao longo do tempo. Observa-se o efeito do valor do bias sobre a turbulência pois para valores mais negativos do bias a amplitude da turbulência é reduzida. Neste caso, $\mathrm{I}_{\text {sat }}$ foi medida em uma sonda posicionada em $\mathrm{R}=1,110 \mathrm{~m}$ e $\mathrm{z}=1,747 \mathrm{~m}$.

\subsection{Descargas analisadas}

Os dados utilizados neste trabalho, foram obtidos no Texas Helimak do Institute for Fusion Studies - IFS, da Universidade do Texas, em Austin. Esta máquina foi desenvolvida e é operada pela equipe coordenada pelo Prof. Dr. Kenneth W. Gentle.

Durante o projeto de doutoramento o doutorando, Dennis L. Toufen, realizou dois estágios no Texas Helimak: de 10/01/11 à 10/02/11 e de 09/01/12 à 30/01/12. Nestes estágios a máquina pode ser conhecida com mais detalhes pelo estudante e foram propostas e realizadas duas experiências: disparos de 110202001 a 110209020.

Parte dos dados aqui utilizados foi obtida pela equipe do Prof. Gentle e a outra durante os estágios realizados no Texas Helimak. A análise destes dados neste trabalho ocorre, portanto, em colaboração com o Prof. Gentle. Em todos os dados utilizados neste trabalho o plasma é constituído de gás argônio com pressão de $1 \times 10^{-5}$ Torr e compreendem disparos realizados de 2008 a 2012. 


\section{MÉTODOS DE ANÁLISE}

Neste capítulo são descritos os métodos utilizados nesta tese para a análise dos dados obtidos através dos sistemas de diagnósticos no Texas Helimak. Essa análise envolve a utilização de ferramentas estatísticas para a análise das flutuações dos sinais medidos, e de análise espectral, linear e não linear (bi-espectral). Também o cálculo do transporte turbulento de partículas, um dos principais objetivos de estudo desta tese, está aqui explicitado. Outro método descrito é a análise de recorrência, utilizada no capítulo 6 como uma ferramenta adicional para caracterização da turbulência. A técnica para determinação da temperatura eletrônica através das medidas da curva característica da sonda de Langmuir é descrita neste capítulo. Por fim são discutidas as medidas espectroscópicas de velocidade de fluxo do plasma e sua análise.

\subsection{Análise estatística das flutuações}

A análise estatística das flutuações presentes no plasma é uma ferramenta útil para análisar as amostras de um sinal constituído por uma série temporal, como mostrado por ASAKURA (2009) e LABIT (2007). Para quantificar o valor médio das flutuações para um sinal em tempo discreto $\mathrm{x}(t)$, no intervalo de tempo entre $t_{1}$ e $t_{n}$, utiliza-se, a grandeza denominada nível de turbulência (turbulence level), que para a corrente de saturação iônica é dada por:

nível de turbulência $=\frac{\sigma}{|\langle\mathrm{x}(t)\rangle|}$,

sendo que \langle\rangle representa a média no tempo e $\sigma$ o desvio padrão,

$$
\sigma=\sqrt{\frac{\sum_{i=1}^{n}\left(\mathrm{x}\left(t_{i}\right)-\langle\mathrm{x}(t)\rangle\right)^{2}}{(n-1)}} .
$$


Para obter informações sobre a simetria das oscilações em torno da média e a importância dos eventos extremos, utiliza-se a skewness (assimetria) e a kurtosis (curtose), respectivamente (NIST/SEMATECH, 2012):

$$
\begin{aligned}
& \text { skewness }=\frac{1}{(n-1)} \frac{\sum_{i=1}^{n}\left(\mathrm{x}\left(t_{i}\right)-\langle\mathrm{x}(t)\rangle\right)^{3}}{\sigma^{3}}, \\
& \text { kurtosis }=\frac{1}{(n-1)} \frac{\sum_{i=1}^{n}\left(\mathrm{x}\left(t_{i}\right)-\langle\mathrm{x}(t)\rangle\right)^{4}}{\sigma^{4}} .
\end{aligned}
$$

Vale lembrar que os valores esperados da skewness e da kurtosis para uma distribuição Gaussiana são, respectivamente, zero e três. Como exemplo das funções introduzidas, a Figura 3.1 mostra o perfil radial da média (para janelas, de $100 \mathrm{~ms}$, consideradas na série temporal analisada), do nível de turbulência, da skewness e da kurtosis para corrente de saturação iônica medida em $\mathrm{z}=1,747 \mathrm{~m}$.
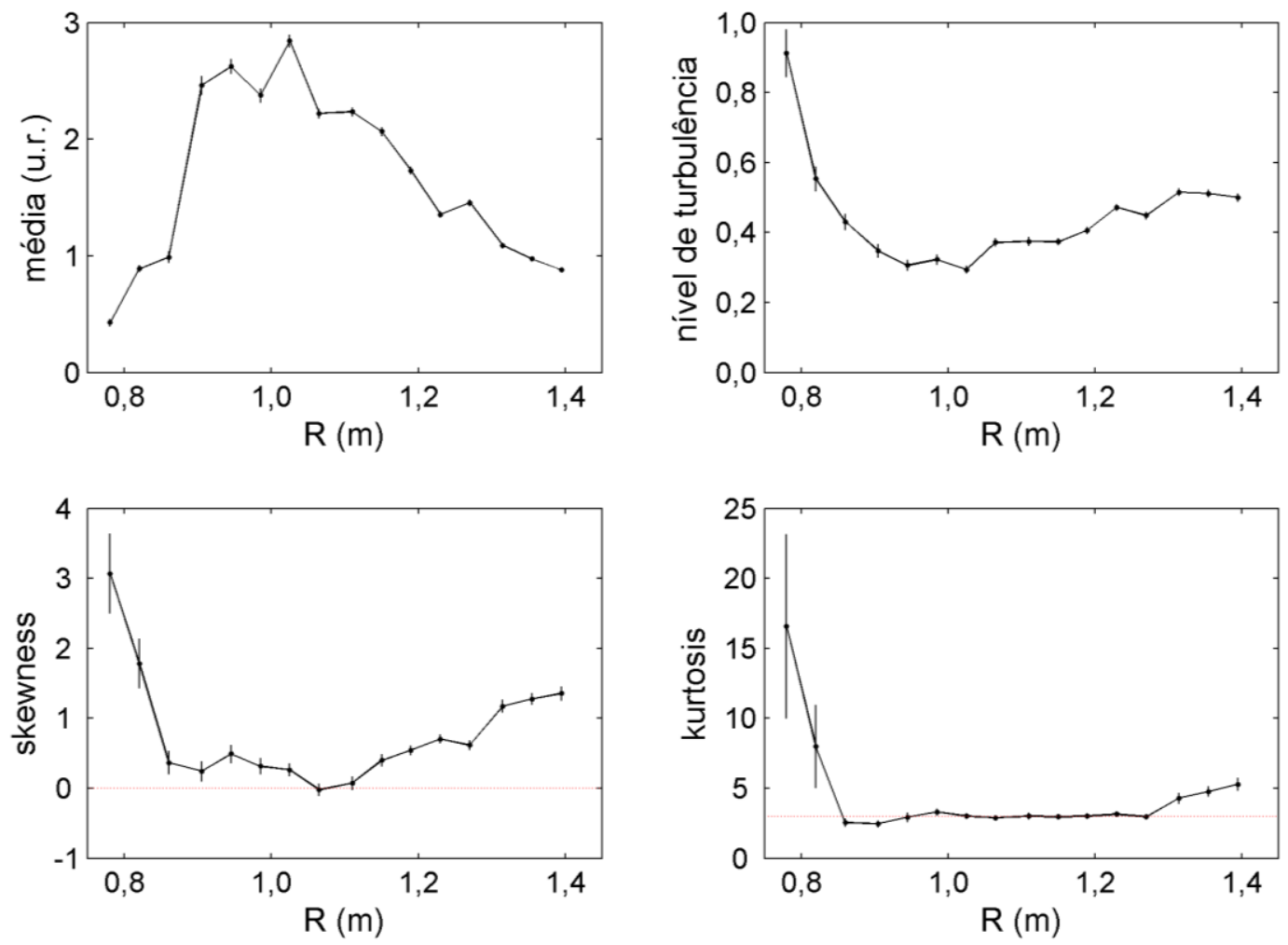

Figura 3.1: Perfis radiais de quatro grandezas estatísticas (média, nível de turbulência, skewness e kurtosis) da corrente de saturação iônica para $\mathrm{z}=1,747 \mathrm{~m}$. As linhas pontilhadas vermelhas marcam os valores de skewness e kurtosis da distribuição gaussiana. 


\subsection{Análise espectral}

A análise espectral do plasma pode fornecer informações valiosas com relação aos fenômenos de turbulência e ondas (RITZ, 1988 e FERREIRA, 1999). Dentro desta análise, além do espectro de potência calculado para cada sonda, pode-se utilizar métodos que, utilizando a informação de duas ou mais sondas, fornecem informação relacionada à propagação de ondas no plasma.

Neste trabalho, a análise espectral foi feita através da chamada transformada de Fourier janelada: divide-se as séries temporais em $N$ realizações, e aplica-se a transformada de Fourier para cada uma destas realizações $j$. Sendo assim, as equações para o espectro de potência $P_{x x}^{j}(f)$ e o espectro cruzado (crosspower spectrum) $C_{x y}^{j}(f)$ para uma dada realização $j$ e uma frequiência $f$ são (LIN, 1991):

$$
\begin{aligned}
& P_{x x}^{j}(f)=X^{j}(f) X^{j} *(f), \\
& C^{j}{ }_{x y}(f)=X^{j}(f) Y^{j} *(f),
\end{aligned}
$$

sendo: $X^{j}(f)$ e $Y^{j}(f)$ as transformadas de Fourier dos sinais $x(t)$ e $y(t)$ para a realização $j, X^{j *}(f)$ e $Y^{j *}(f)$ os complexos conjugados de $X^{j}(f)$ e $Y^{j}(f)$.

Na Figura 3.2, observa-se um sinal de corrente de saturação iônica do Texas Helimak, e seu espectro de potência. 

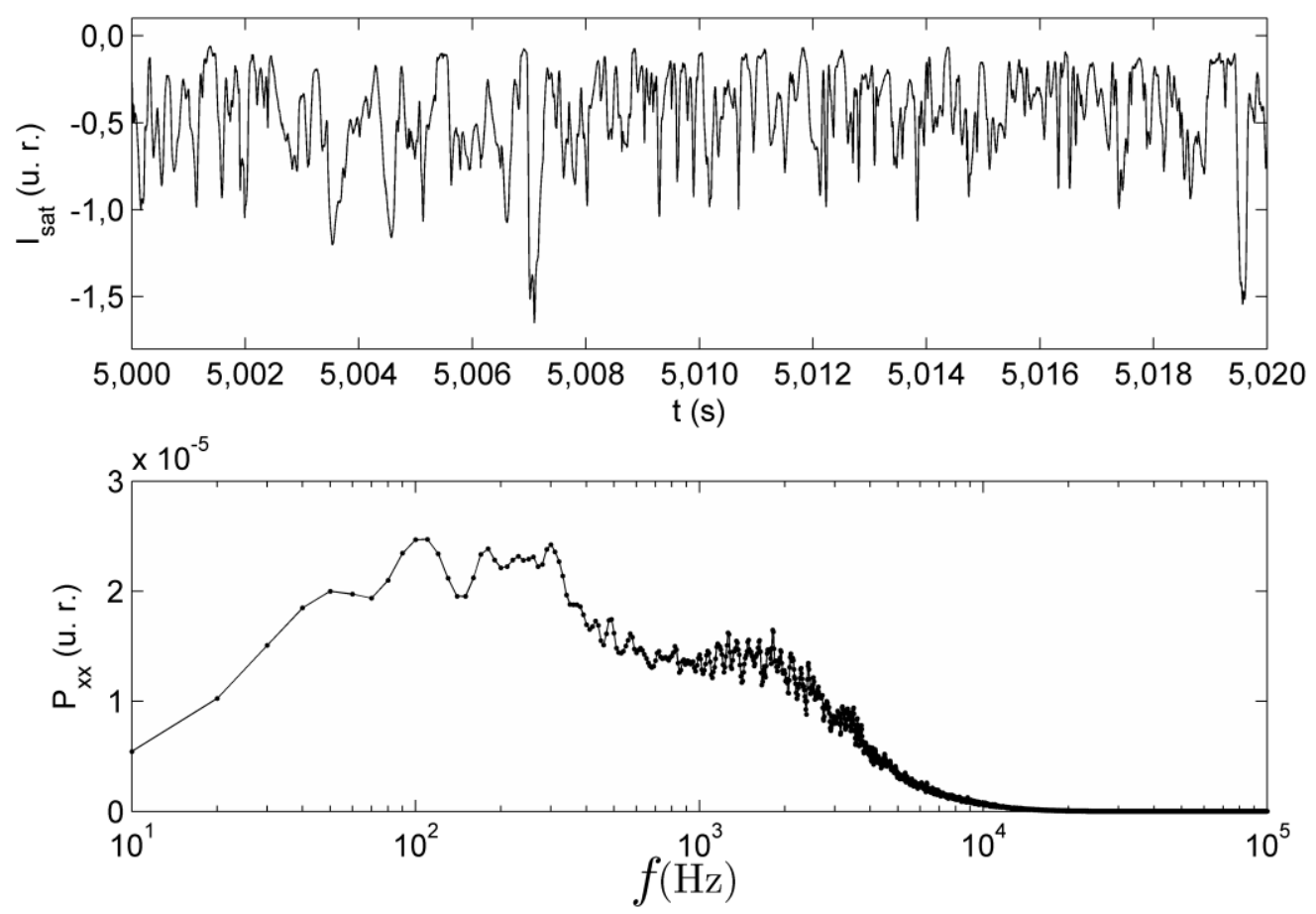

Figura 3.2: Exemplo de sinal de corrente de saturação iônica em função do tempo (acima) e espectro de potência (abaixo). Neste exemplo a sonda estava localizada em $\mathrm{R}=1,210 \mathrm{~m} \mathrm{e} \mathrm{z}=1,747 \mathrm{~m}$.

Como $C^{j}{ }_{x y}(f)$ é uma grandeza complexa, esta pode ser escrita de forma a explicitar a diferença de fase entre os sinais $\mathrm{x}(\mathrm{t})$ e $\mathrm{y}(\mathrm{t}), \theta^{j}(f)$ :

$$
C_{x y}^{j}(f)=\left|C_{x y}^{j}(f)\right| e^{i \theta^{j}(f)} .
$$

Utilizando a forma apresentada na Equação 3.7, pode-se estimar o número de onda $k^{j}(f)$, utilizando-se dois sinais medidos em duas posições próximas como sendo (RITZ, 1988):

$$
k^{j}(f)=\frac{\theta^{j}(f)}{\Delta d},
$$

onde $\Delta d$ é a distância entre as sondas que coletam os sinais $\mathrm{x}(\mathrm{t})$ e $\mathrm{y}(\mathrm{t})$.

A Figura 3.3 mostra exemplos do número de onda médio em função da frequência, $k(f)$, para duas sondas, medindo corrente de saturação iônica, localizadas no mesmo raio e com quatro diferentes valores de $\Delta \mathrm{z}(2 \mathrm{~cm}, 4 \mathrm{~cm}, 6 \mathrm{~cm}$ e $8 \mathrm{~cm}) . O$ $k(f)$ fornece informação sobre o número de onda e indica a velocidade de fase, mas não fornece a potência do sinal. Nos espectros para $\Delta z=6 \mathrm{~cm}$ e $8 \mathrm{~cm}$ foi utilizada uma correção para pontos com $|\theta(f)|>2 \pi$ fossem posicionados na mesma reta. 

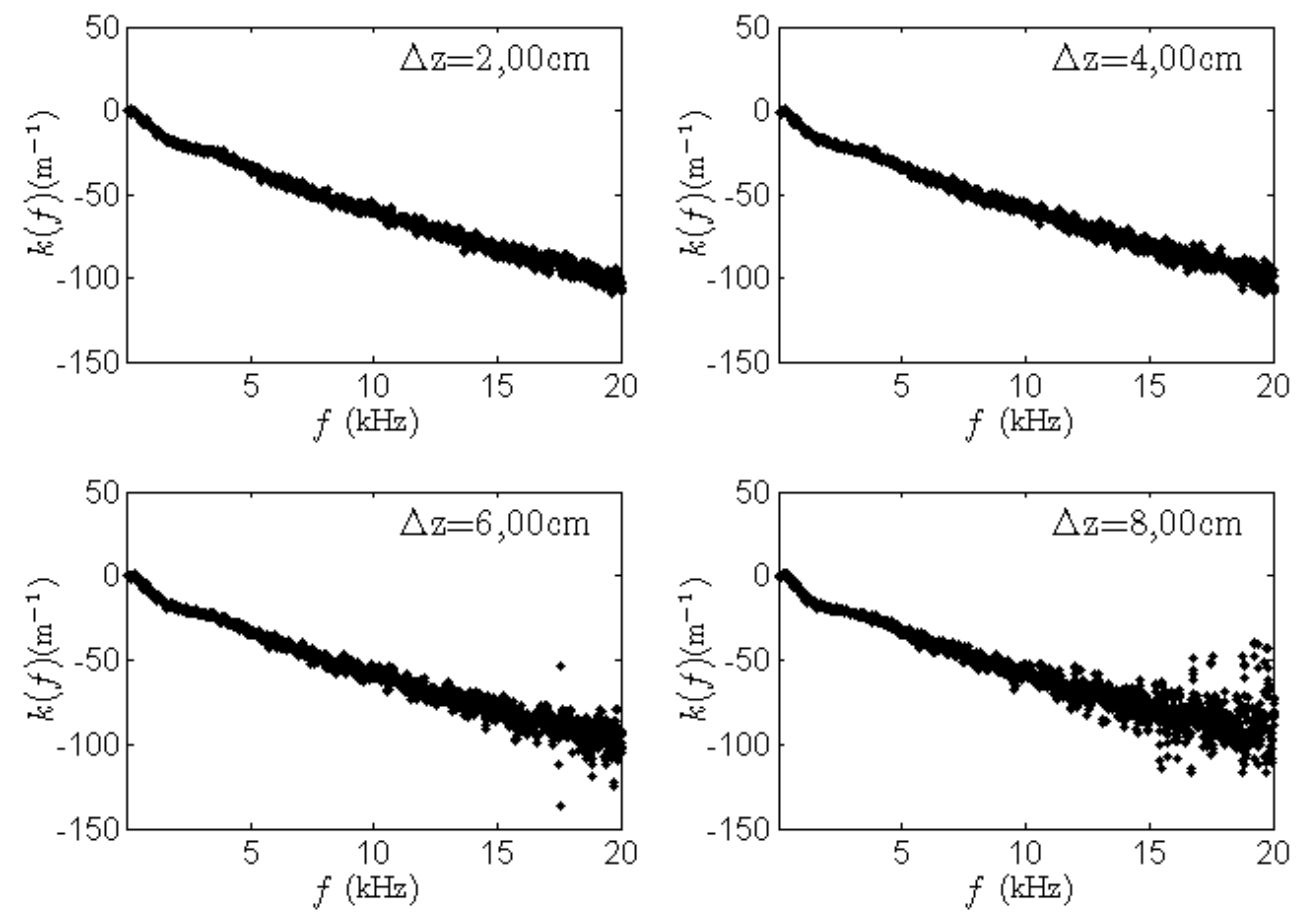

Figura 3.3: Exemplos de números de onda médios em função da frequência, $k(f)$, para pares de sondas medindo corrente de saturação iônica. Neste exemplo fixou-se uma sonda $(R=1,210 \mathrm{~m} \mathrm{e} \mathrm{z}=1,747 \mathrm{~m}) \mathrm{e}$ foram utilizadas outras quatro sondas com a mesma posição radial $(\mathrm{R}=1,210 \mathrm{~m})$ e diferentes alturas $(\mathrm{z}=$ $1,767 \mathrm{~m}, 1,787 \mathrm{~m}, 1,807 \mathrm{~m}$ e $1,827 \mathrm{~m})$. Nos espectros para $\Delta \mathrm{z}=6 \mathrm{~cm}$ e $8 \mathrm{~cm}$ foi utilizada uma correção para pontos com $|\theta(f)|>2 \pi$ fossem posicionados na mesma reta.

A potência, relacionada tanto à frequência quanto ao número de onda, é fornecida pelo espectro de potência $S(k, f)$ estimado somando-se $\left|C^{j}{ }_{x y}(f)\right|$ para as frequências $f$ e número de onda $k$ :

$$
S(k, f)=\sum_{j} \delta_{k, k^{j}} \delta_{f, f^{j}}\left|C^{j}{ }_{x y}(f)\right| .
$$

Um exemplo de espectro de $S(k, f)$, para as mesmas condições do exemplo da Figura 3.3, pode ser observado na Figura 3.4. Esse espectro pode ser utilizado para estimar a velocidade de fase média ponderando a razão $f / k$ pela potência dada pelo valor de $S(k, f)$ :

$$
V_{p h}=\frac{\sum_{f} \sum_{k}\left(S(k, f) \cdot \frac{f}{k}\right)}{\sum_{f} \sum_{k} S(k, f)} .
$$


A inclinação da reta em preto, vista na Figura 3.4, indica a velocidade de fase média estimada para este exemplo $\left(V_{p h} \cong-100 \mathrm{~m} / \mathrm{s}\right)$. No espectro de $S(k, f)$, ainda é possível identificar para quais valores de $k$ e de $f$ existe mais potência concentrada. No caso no exemplo percebe-se uma maior concentração de potência para $f \cong 100 \mathrm{~Hz}$ (mancha vermelha).

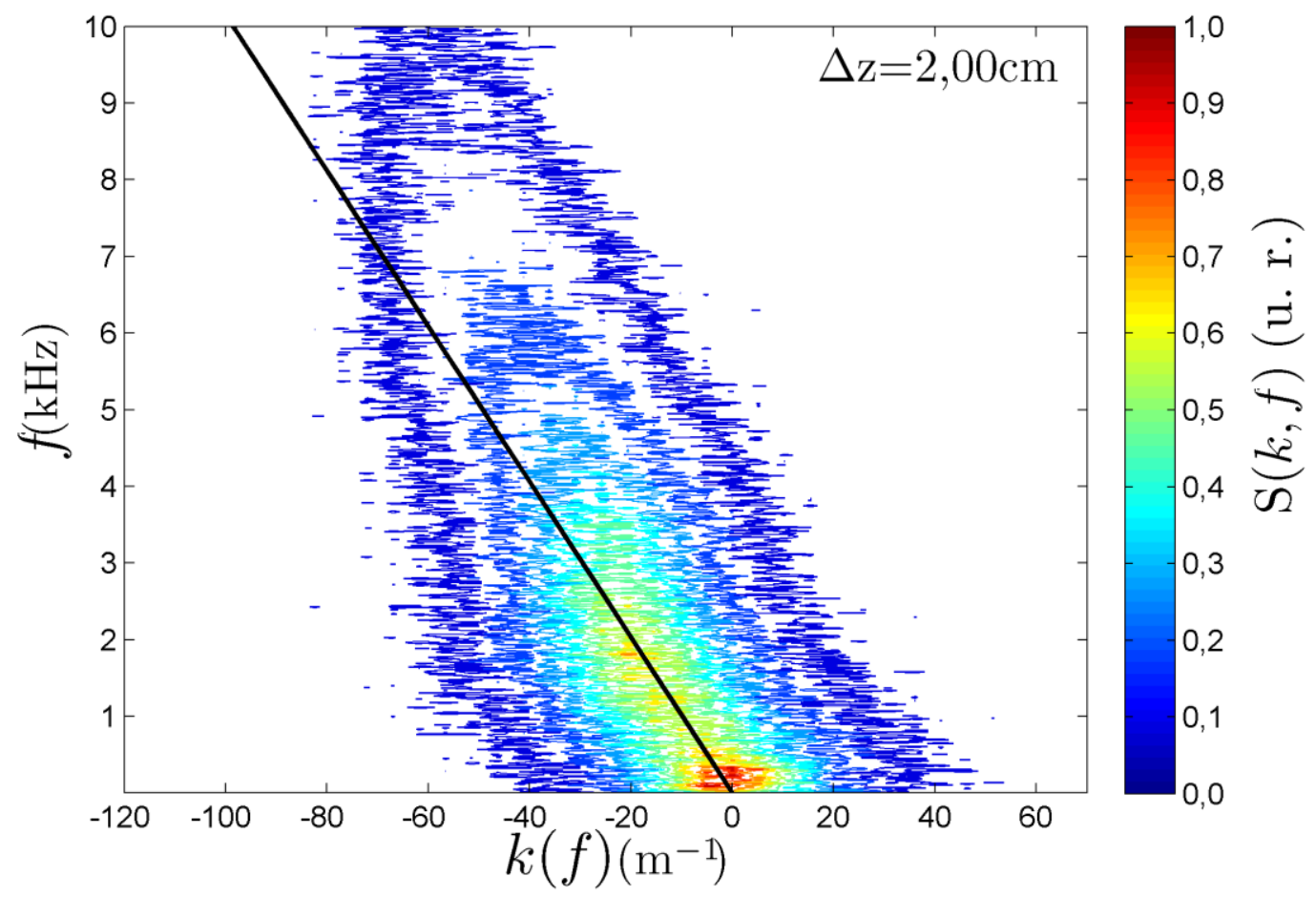

Figura 3.4: Exemplo de espectro de $S(k, f)$ para duas sondas medindo corrente de saturação iônica. A reta em preto representa a velocidade de fase estimada (neste exemplo: $V_{p h} \cong-100 \mathrm{~m} / \mathrm{s}$ ). Neste exemplo as sondas estavam localizadas no mesmo raio $(\mathrm{R}=1,210 \mathrm{~m})$ e diferentes valores de $\mathrm{z}(\mathrm{z}=1,747 \mathrm{~m} \mathrm{e}$ $\mathrm{z}=1,767 \mathrm{~m})$.

Outro espectro importante é o de coerência, que é calculado como (POWERS, 1990):

$$
\gamma^{2}(f)=\frac{\left|\left\langle C_{x y}^{j}(f)\right\rangle\right|^{2}}{\left\langle P_{x x}^{j}(f)\right\rangle\left\langle P^{j}{ }_{y y}(f)\right\rangle},
$$

sendo que \langle\rangle , denota a média sobre todas as $\mathrm{N}$ realizações $j$. A Figura 3.5 mostra os espectro de coerência para as mesmas condições dos espectros de $k(f)$ mostrados na Figura 3.3. Nota-se que quanto mais afastadas estão as sondas, menor é a coerência. Nota-se também que a coerência cai menos para as frequências em torno do pico em 
$f \cong 100 \mathrm{~Hz}$. Neste trabalho a maioria das análises será realiza com sondas separadas verticalmente por $2 \mathrm{~cm}(\Delta \mathrm{z}=2 \mathrm{~cm})$, a Figura 3.5 mostra que para esses casos a coerência apresenta valores altos para $f \leq 5 \mathrm{kHz}$.
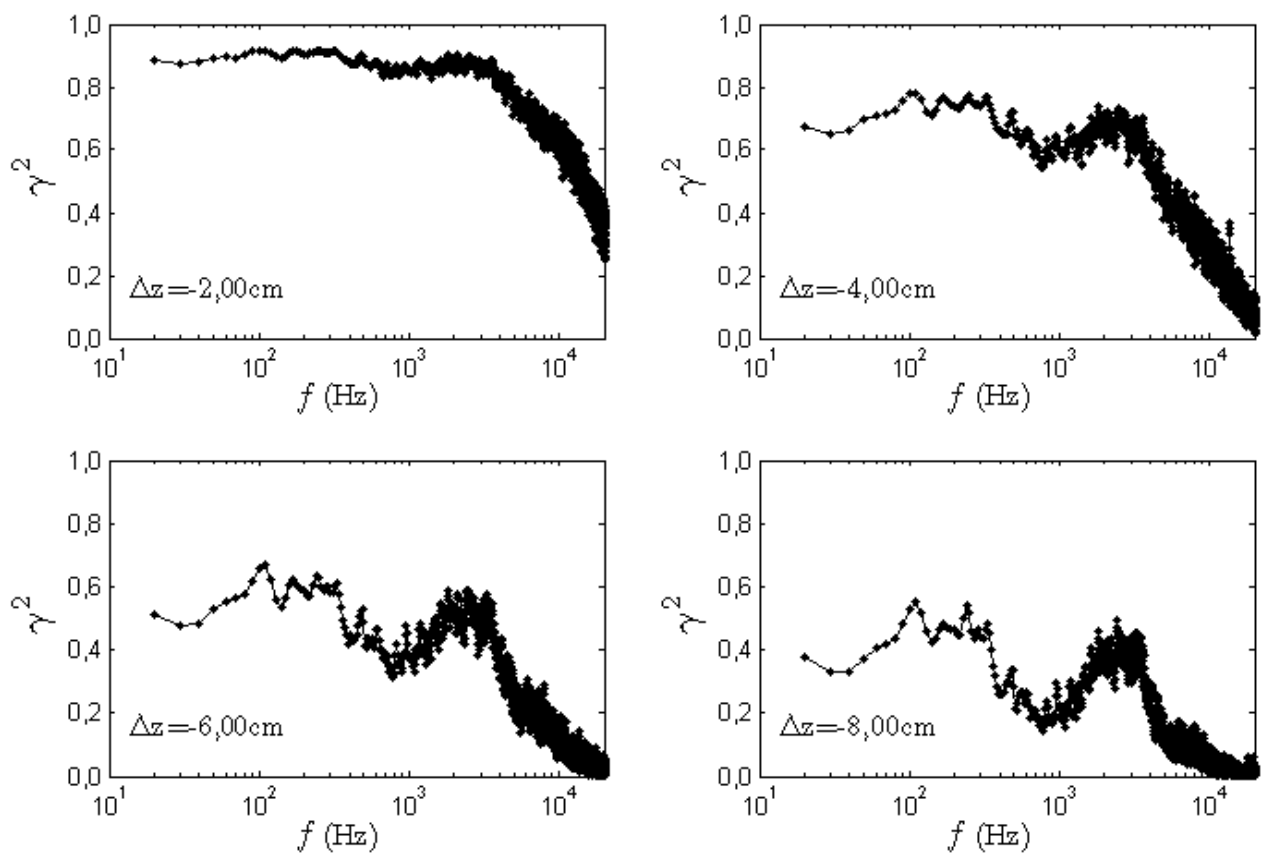

Figura 3.5: Exemplo de espectros de coerência para pares de sondas medindo corrente de saturação iônica. Neste exemplo fixou-se uma sonda $(\mathrm{R}=1,210 \mathrm{~m} \mathrm{e} \mathrm{z}=1,747 \mathrm{~m})$ e foram utilizadas outras quatro sondas com a mesma posição radial $(\mathrm{R}=1,210 \mathrm{~m})$ e diferentes alturas $(\mathrm{z}=1,767 \mathrm{~m}, 1,787 \mathrm{~m}, 1,807 \mathrm{~m} \mathrm{e}$ $1,827 \mathrm{~m})$.

\subsubsection{Análise bi-espectral}

Em determinadas circunstâncias é importante identificar não só a presença de modos e suas características, mas também como estes se acoplam entre si. Um dos procedimentos mais bem sucedidos para identificar o acoplamento de modos e estimar a sua importância é a análise bi-espectral. (RITZ, 1988; VAN MILLIGEN, 2008 e DOS SANTOS LIMA, 2009-2).

Considerando um plasma cujo espectro de potência da turbulência é semelhante ao mostrado na Figura 3.2, pode-se aplicar o procedimento padrão de análise biespectral (HIDALGO, 1993; VAN MILLIGEN, 1995; KIM, 1978) com o objetivo de identificar e medir o acoplamento não linear (quadrático) entre modos. Para estimar a importância deste acoplamento, a auto-bicoerência pode ser calculada através do autobiespectro (KIM, 1980) como feito em (DOS SANTOS LIMA, 2009-2). 
Tomando $\phi_{n 1}(f)$ como a transformada discreta de Fourier da série temporal $n 1(t)$ na frequência $f$ o auto-biespectro de $n 1(t)$ será dado por:

$S_{n 1 n 1 n 1}\left(f_{1}, f_{2}\right)=\left\langle\phi_{n 1}\left(f_{3}\right) \phi_{n 1}^{*}\left(f_{1}\right) \phi_{n 1}^{*}\left(f_{2}\right)\right\rangle$,

onde $f_{3}=f_{1}+f_{2}$.

O auto-biespectro contém informação a respeito do acoplamento não-linear de modos com frequências $f_{1}, f_{2}$ e $f_{3}$. Porém, sua amplitude depende tanto da intensidade do acoplamento como da potência do sinal nas frequências dadas. A normalização do auto-biespectro pela potência do sinal resulta na auto-bicoerência, $b_{n 1 n 1 n 1}^{2}$ ou simplesmente $b^{2}$, a qual não depende da amplitude do sinal:

$b^{2}\left(f_{1}, f_{2}\right)=\frac{\left|S_{n 1 n 1 n 1}\left(f_{1}, f_{2}\right)\right|^{2}}{\left\langle\left|\phi_{n 1}\left(f_{1}\right) \phi_{n 1}\left(f_{2}\right)\right|^{2}\right\rangle\left\langle\left|\phi_{n 1}\left(f_{3}\right)\right|^{2}\right\rangle}$.

Na Figura 3.6 é mostrado o espectro de auto-bicoerência para o mesmo sinal cuja evolução temporal e espectro de potência são mostrados na Figura 3.2 (para uma sonda em $\mathrm{R}=1,210 \mathrm{~m}$ e $\mathrm{z}=1,747 \mathrm{~m}$ ). Na Figura 3.6 as frequências $f_{1}$ e $f_{2}$ estão nos eixos e a auto-bicoerência está na escala de cinza. Nesta figura observam-se regiões de maior valor de $b^{2}$, como por exemplo, onde frequências $f_{1} \cong 0,10 \mathrm{kHz}$ e $f_{2} \cong 0,05 \mathrm{kHz}$ Neste exemplo foram graficadas apenas frequências menores ou iguais a $1 \mathrm{kHz}$. No entanto, a menos quando informado o contrário, neste trabalho a auto-bicoerência será calculada com frequências de até $4 \mathrm{kHz}$. 


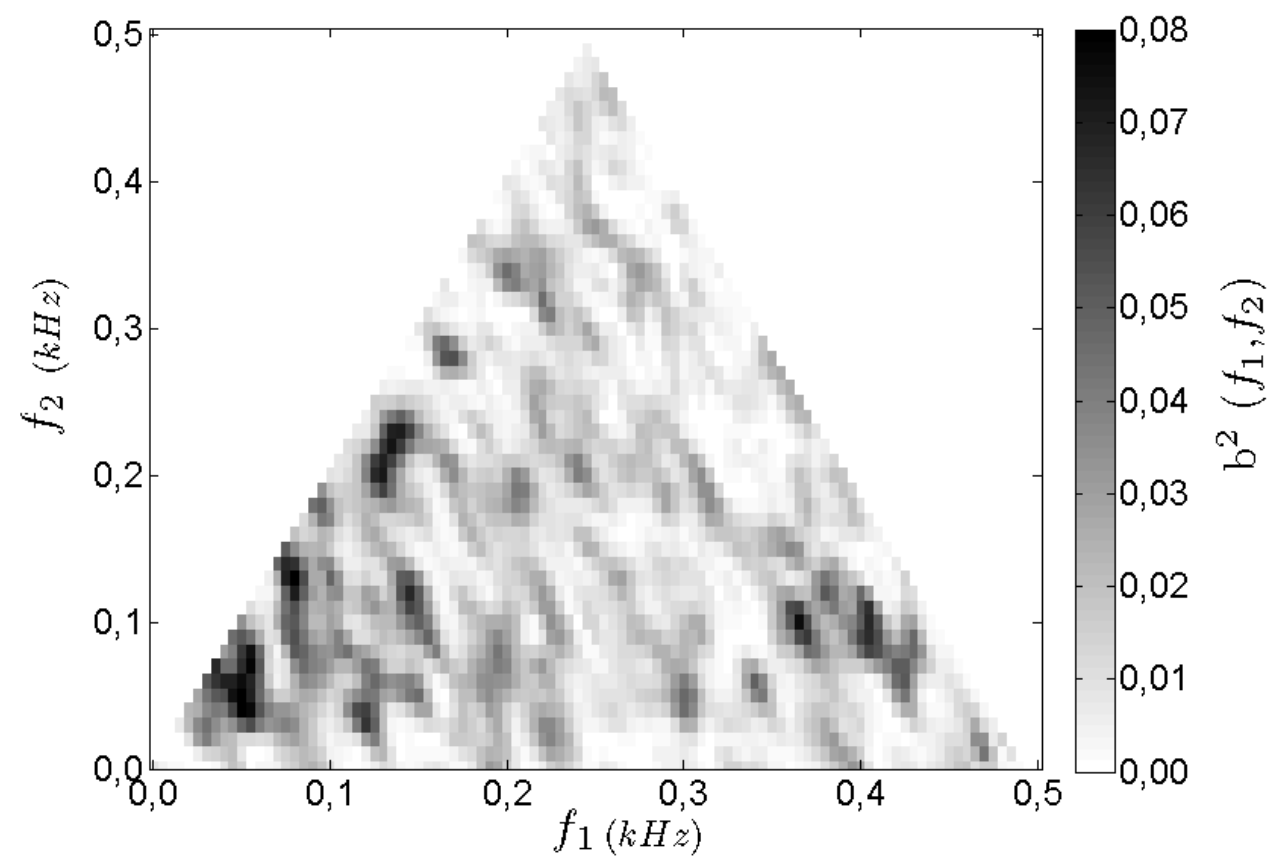

Figura 3.6: Exemplo de espectro de auto-bicoerência para o sinal de corrente de saturação iônica medido pela sonda localizada em $\mathrm{R}=1,210 \mathrm{~m} \mathrm{e} \mathrm{z}=1,747 \mathrm{~m}$. Neste disparo o bias é zero.

\subsection{Cálculo do transporte turbulento}

O fluxo de partículas na direção radial, devido às flutuações eletrostáticas, pode ser calculado como (FERREIRA, 2004):

$$
\Gamma_{r}=\left\langle\tilde{n}(t) \tilde{v}_{r}(t)\right\rangle
$$

sendo que $\tilde{n}$ e $\tilde{v}_{r}$ são, respectivamente, as flutuações de densidade e de velocidade de deriva elétrica na direção radial $\left(v_{r}=\mathbf{v}_{\mathbf{E} \times \mathbf{B}} \cdot \mathbf{e}_{r}\right)$, ou seja, os valores de $n$ e $v_{r}$ subtraídos de seus valores médios.

Lembrando da relação da velocidade de deriva elétrica com o campo elétrico (CHEN, 1984):

$$
\mathbf{v}_{E x B}=\frac{\mathbf{E} \times \mathbf{B}}{\mathbf{B}^{2}},
$$

e da corrente de saturação iônica com a densidade, 


$$
I_{s a t}=\alpha A e n_{e} \sqrt{\frac{T_{e}}{m_{i}}},
$$

é possível escrever o transporte de partículas em flutuações eletrostáticas (RITZ, 1988) como:

$$
\Gamma_{\mathrm{r}} \propto\left\langle\tilde{I}_{s a t} \tilde{E}_{z}\right\rangle_{t}
$$

Sendo que \langle\rangle$_{t}$ denota a média no tempo. O campo elétrico na direção vertical, $E_{z}$, é calculado como:

$$
E_{z} \cong-\frac{\Delta \phi_{p}}{\Delta z},
$$

onde $\Delta z=z_{1}-z_{2}$ é a diferença da posição vertical entre as duas sondas e $\Delta \phi_{p}$ é a diferença do potencial do plasma nas posições $z_{1}$ e $z_{2}$. Esta diferença é tomada como aproximadamente igual à diferença entre os potencias flutuantes medidos nas posições $z_{1}$ e $z_{2}$, já que a relação entre o potencial do plasma, $\phi_{p}$, e o potencial flutuante, $\phi_{f}$, é (LIN, 1991):

$$
\phi_{p} \cong \beta T_{e}+\phi_{f}
$$

sendo $T_{e}$ a temperatura dos elétrons, $\beta$ uma constante cujo valor depende das condições do plasma. Da mesma forma que o encontrado na literatura (RITZ, 1988), essa equação será tomada como uma boa aproximação para o plasma de Argônio analisado nesta tese.

Com isso, assumindo a temperatura dos elétrons no plasma aproximadamente uniforme pode-se utilizar a aproximação:

$$
\Delta \phi_{f} \cong \Delta \phi_{p} \text {. }
$$

Assim, o campo elétrico na direção vertical é calculado como:

$$
E_{z} \cong-\frac{\Delta \phi_{f}}{\Delta z},
$$

e com isso o fluxo radial de partículas pode ser estimado experimentalmente como:

$$
\Gamma_{\mathrm{r}} \propto\left\langle\frac{\tilde{I}_{s a t} \widetilde{\Delta \phi}_{f}}{\Delta \mathrm{z}}\right\rangle_{t} .
$$




\subsection{Medidas de temperatura do plasma}

A temperatura eletrônica do plasma pode ser medida de diversas maneiras. Uma forma possível é através do ajuste direto da curva característica da sonda, vista na Figura 3.7.

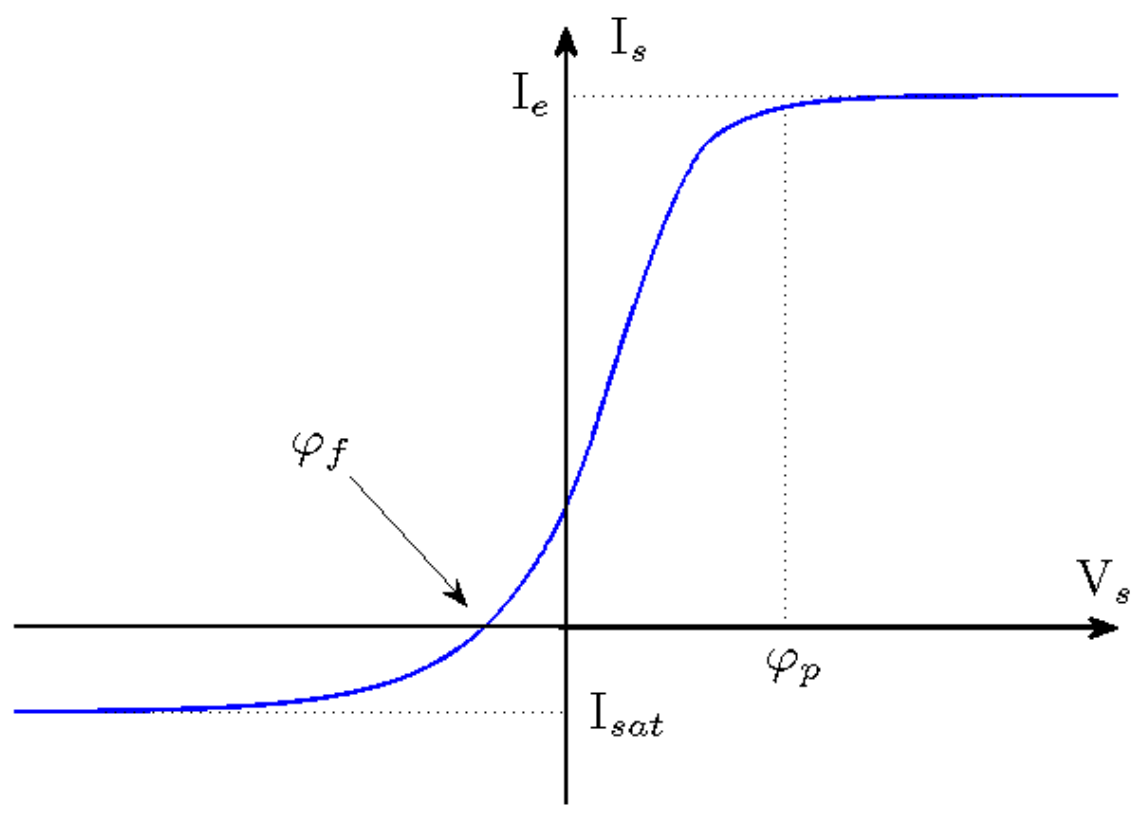

Figura 3.7: Curva característica de uma sonda de Langmuir.

De maneira ideal, a curva característica da sonda é dada por:

$$
I_{s}=I_{s a t}\left(1-\exp \left[\frac{e\left(V_{s}-\varphi_{f}\right)}{k_{B} T_{e}}\right]\right) \quad p / \quad V_{s}<\varphi_{p}
$$

válida apenas para a faixa em que a tensão na sonda $\left(V_{s}\right)$ é menor que o potencial do plasma (CASTRO, 1996).

Conhecendo-se a curva característica de cada sonda é possível determinar o valor da temperatura dos elétrons através de um ajuste exponencial. Porém, nos dados analisados, a parte saturada da curva não é perfeitamente constante. Assim, a curva ajustada para determinar a temperatura eletrônica, 
$I_{s}=\left\{\begin{array}{cc}I_{o}+I_{1}\left(V_{s}-\varphi_{f}\right) & p / V_{s}<\varphi_{f} \\ I_{o}+I_{1}\left(V_{s}-\varphi_{f}\right)+I_{s a t}\left(1-\exp \left[\frac{e\left(V_{s}-\varphi_{f}\right)}{k_{B} T_{e}}\right]\right) & p / \quad \varphi_{f}<V_{s}<\varphi_{p}\end{array}\right.$

ajusta a região de saturação como uma reta de inclinação variável. Um exemplo de ajuste por método dos mínimos quadrados para determinação da temperatura eletrônica é visto na Figura 3.8.

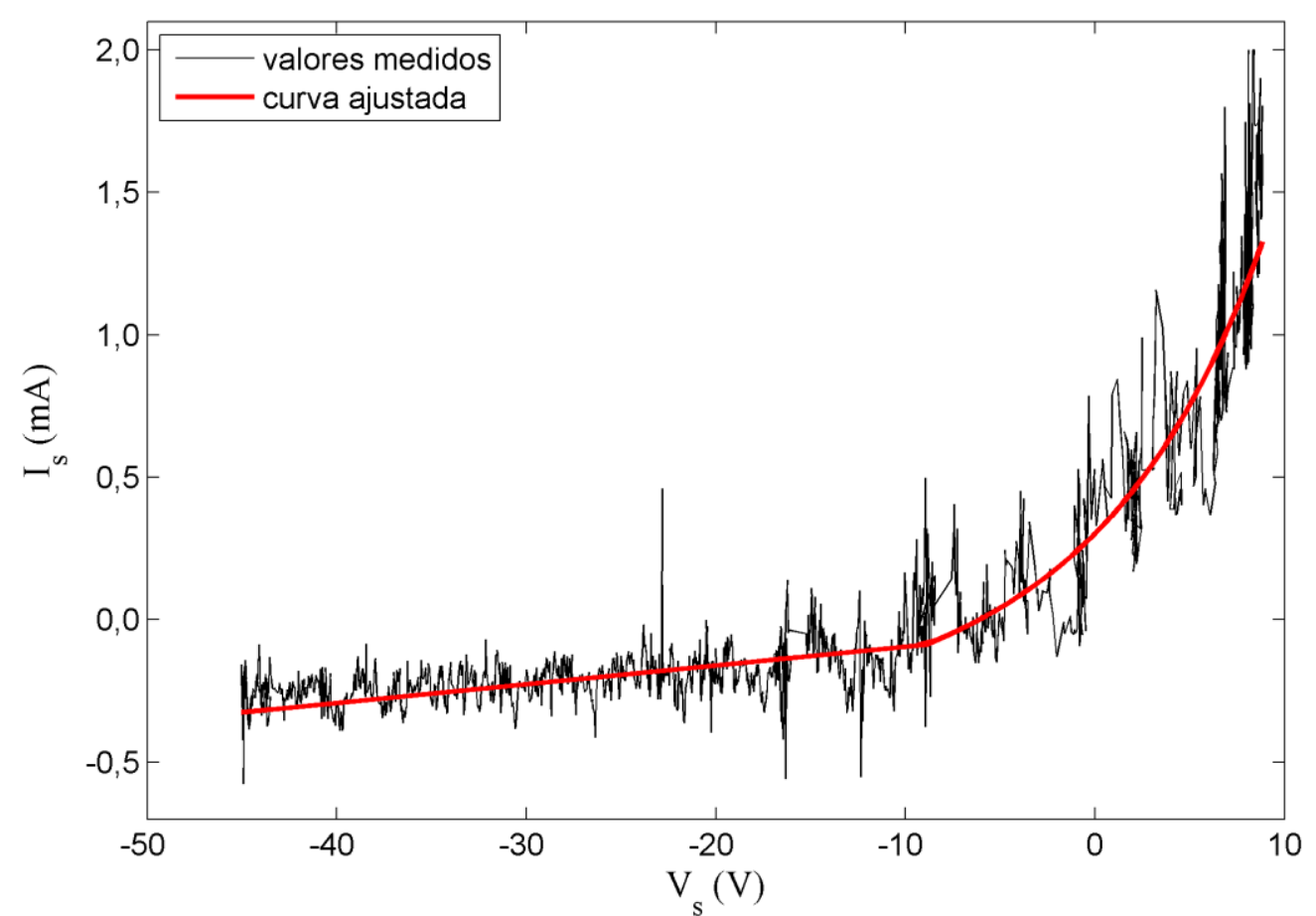

Figura 3.8: Curva característica da sonda de Langmuir (corrente em função do potencial na sonda) ajustada aos dados do Texas Helimak para raio $\mathrm{R}=0,975 \mathrm{~m}$.

\subsection{Análise de recorrência}

A análise de recorrência é um procedimento amplamente utilizado em sistemas dinâmicos (MARWAN, 2002) na qualificação de séries temporais e aplicado nas mais diversas áreas como meteorologia (MARWAN, 2003), geofísica (KURTHS, 1994), plasma espacial $(\mathrm{MARCH}, 2005)$ e plasmas magneticamente confinados (DENDY, 
2006; GUIMARÃES-FILHO， 2010; GUIMARÃES-FILHO， 2008-2). Aqui a recorrência é identificada como um retorno a um dado valor em uma série temporal. Ou seja, dada a série temporal $n(t)$ em tempo discreto, $n\left(t_{j}\right)$ será considerado recorrente à $n\left(t_{i}\right)$ quando a desigualdade a seguir for satisfeita (MARWAN, 2007):

$$
\left\|n\left(t_{i}\right)-n\left(t_{j}\right)\right\|<\epsilon,
$$

onde $\epsilon$ é o limiar de recorrência escolhido para a análise como uma pequena fração do desvio padrão de $n(t)$ e \|| $\|$ representa a norma Euclidiana.

A recorrência de uma dada série temporal pode ser estudada através do diagrama de recorrência (recurrence plot) que é uma representação gráfica da matriz $R_{i j}$ :

$$
R_{i j}=\Theta\left(\epsilon-\left\|n\left(t_{i}\right)-n\left(t_{j}\right)\right\|\right), \quad i, j=1,2, \ldots, N,
$$

onde $\Theta($ ) é função degrau unitário e $\mathrm{N}$ é o numero de pontos analisados da série temporal $n(t)$.

O diagrama de recorrência é desenhado marcando-se um ponto preto se $R_{i j}=1$ e um ponto branco se $R_{i j}=0$. A Figura 3.9 (a) mostra um exemplo de diagrama de recorrência para um sinal senoidal que, devido ao caráter periódico da série temporal, mostra linhas diagonais perfeitas. Já a Figura 3.9 (b) mostra o diagrama de recorrência de um sinal randômico. Nota-se que neste caso o diagrama não apresenta nenhuma estrutura o que é esperado uma vez que os pontos da série temporal não devem apresentar nenhum tipo de dependência com os demais pontos da série. 

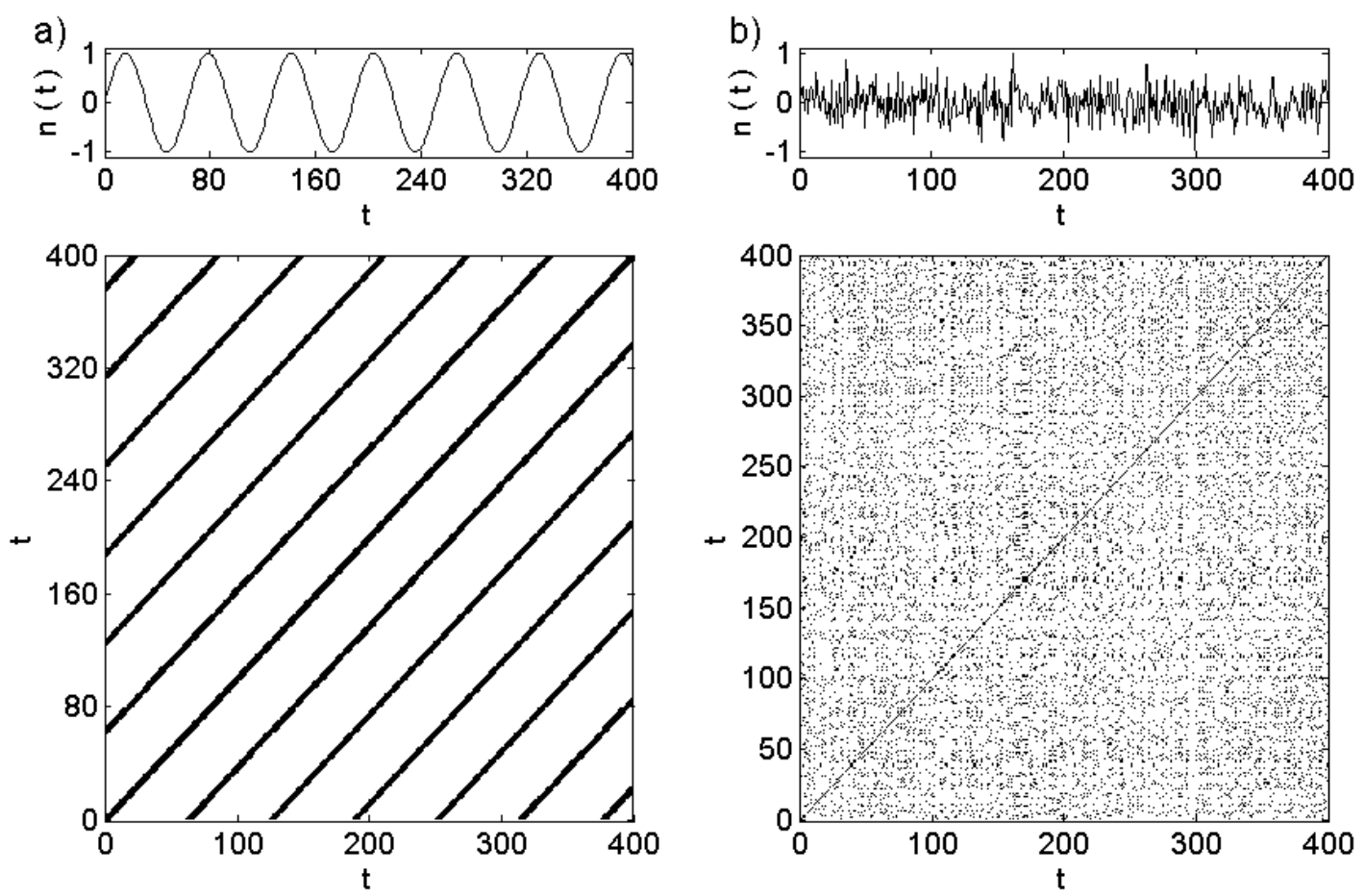

Figura 3.9: Exemplos de diagramas de recorrência para (a) um sinal senoidal e (b) um sinal aleatório.

O diagrama de recorrência fornece uma ideia qualitativa da relação entre os pontos da série temporal, no entanto, para determinadas situações, é interessante quantificar a recorrência. Essa análise (Recurrence Quantification Analysis - RQA) pode incluir a determinação de uma série de parâmetros a partir do diagrama de recorrência, como a taxa de recorrência (recurrence rate - RR), o determinismo (DET), a laminaridade (LAM) entre outros. Neste trabalho foi utilizado apenas o determinismo, que é uma estimativa da fração dos pontos do diagrama que pertencem a uma diagonal e pode ser calculado como mostrado na equação 3.27 (MARWAN, 2007),

$$
D E T=\frac{\sum_{\ell=\ell_{\min }}^{\ell_{\max }} \ell P(\ell)}{\sum_{i, j=1}^{N} R_{i, j}, \operatorname{para} i \neq j},
$$

onde $\ell$ é o comprimentos de cada umas das diagonais do diagrama que são paralelas a diagonal principal e $P(\ell)$ é a frequência na qual uma diagonal com comprimento $\ell$ aparece no diagrama.

Na Figura 3.10 é visto um exemplo de diagrama de recorrência para um sinal de corrente de saturação iônica do Texas Helimak. Nela, observa-se a diagonal principal que sempre deve existir pois um instante de tempo será sempre recorrente com ele 
mesmo. Além disso, nota-se que o diagrama de recorrência deste exemplo apresenta estruturas como as vistas entre os instantes 2 e $3 \mathrm{~ms}$, mostrando que a recorrência do plasma é um caso intermediário entre os dois mostrados no exemplo da Figura 3.9.

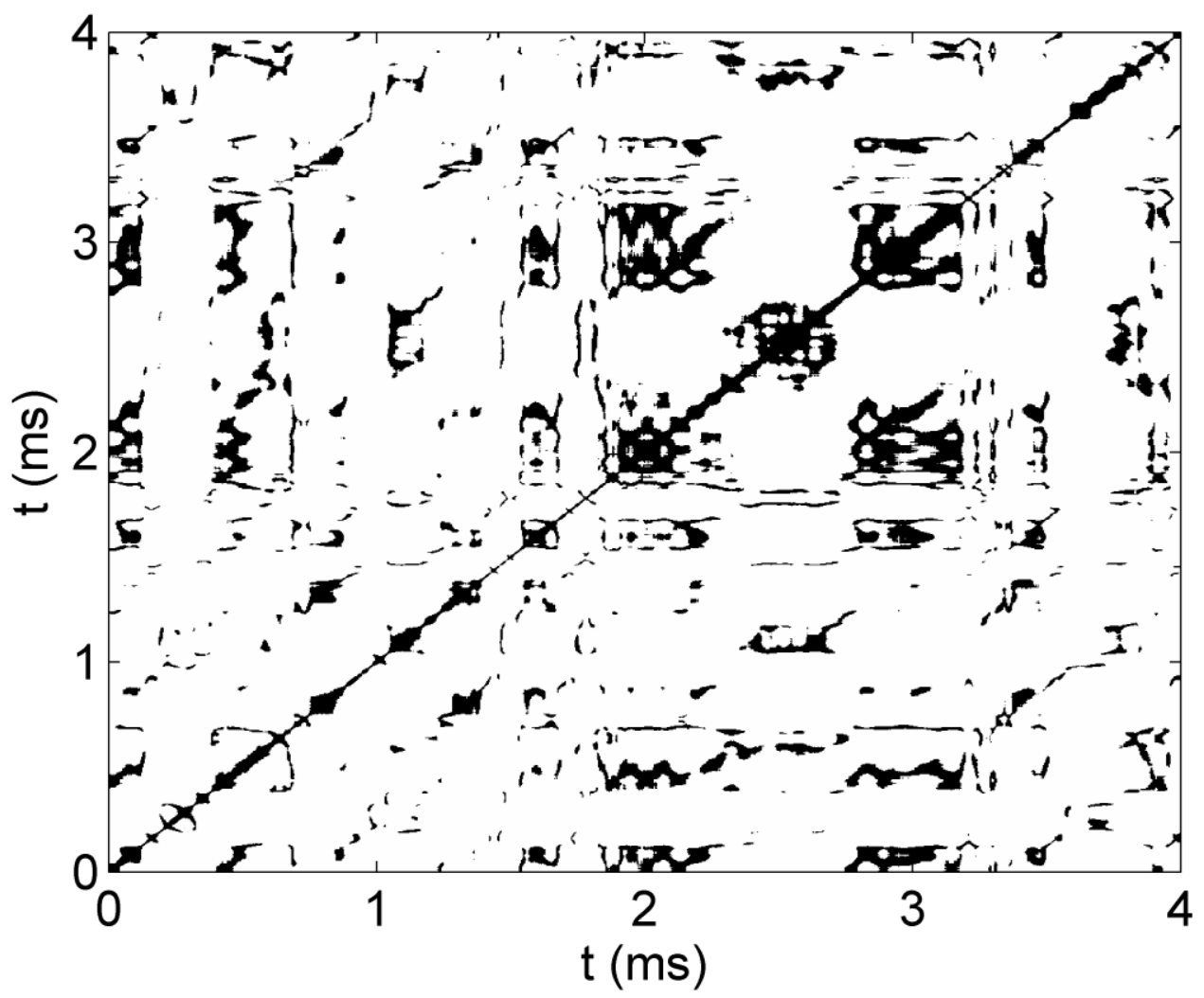

Figura 3.10: Exemplo de diagrama de recorrência para um série de corrente de saturação iônica medida em uma sonda posicionada em $\mathrm{R}=1,190, \mathrm{z}=1,747$ e disparo com valor de bias igual a zero. Para esse diagrama o valor do determinismo estimado foi $\mathrm{DET}=0,97(1)$.

\subsection{Medidas de velocidade de fluxo vertical do plasma $V_{z}$}

Como já mencionado no capítulo 2, o Texas Helimak utiliza um espectrômetro para medir a velocidade vertical do fluxo de plasma através do deslocamento Doppler das linhas de emissão do íon, Argônio em geral. Estas medidas utilizam uma técnica de análise semelhante à descrita por ROWAN (2008). Este espectrômetro é operado por uma equipe dedicada e seus dados são adquiridos pelos mesmos ADCs utilizados para aquisição dos sinais das sondas de Langmuir, de modo que as medidas de velocidade $V_{z}$ e das sondas devem ser tomadas em disparos diferentes. A incerteza típica das medidas espectroscópicas de velocidade é da ordem de 50 m/s (GENTLE, 2010). Como os dados 
espectroscópicos são tratados pela mesma equipe que opera o espectrômetro, os perfis de $V_{z}$ foram cedidos para serem utilizados neste trabalho já previamente tratados pela equipe do Prof. Rowan.

Além da velocidade do plasma, uma grandeza muito estudada devido a sua relação com o transporte (BIGLARI, 1990) é o cisalhamento (shear) da velocidade, que no caso do helimak é definido como o gradiente radial da velocidade vertical (LI, 2011): $d V_{z} / d R$. A Figura 3.11 mostra exemplos de perfis radiais da velocidade vertical de fluxo do plasma, $V_{z}$, e do cisalhamento. Observa-se nestes exemplos que a velocidade apresenta um ponto de cisalhamento nulo aproximadamente em $\mathrm{R}=1,250 \mathrm{~m}$. Regiões sem cisalhamento (shearless) têm sido amplamente estudadas devido a previsões teóricas ligadas a existência de barreiras de transporte robustas na região sem cisalhamento (DEL-CASTILLO-NEGRETE, 2000).
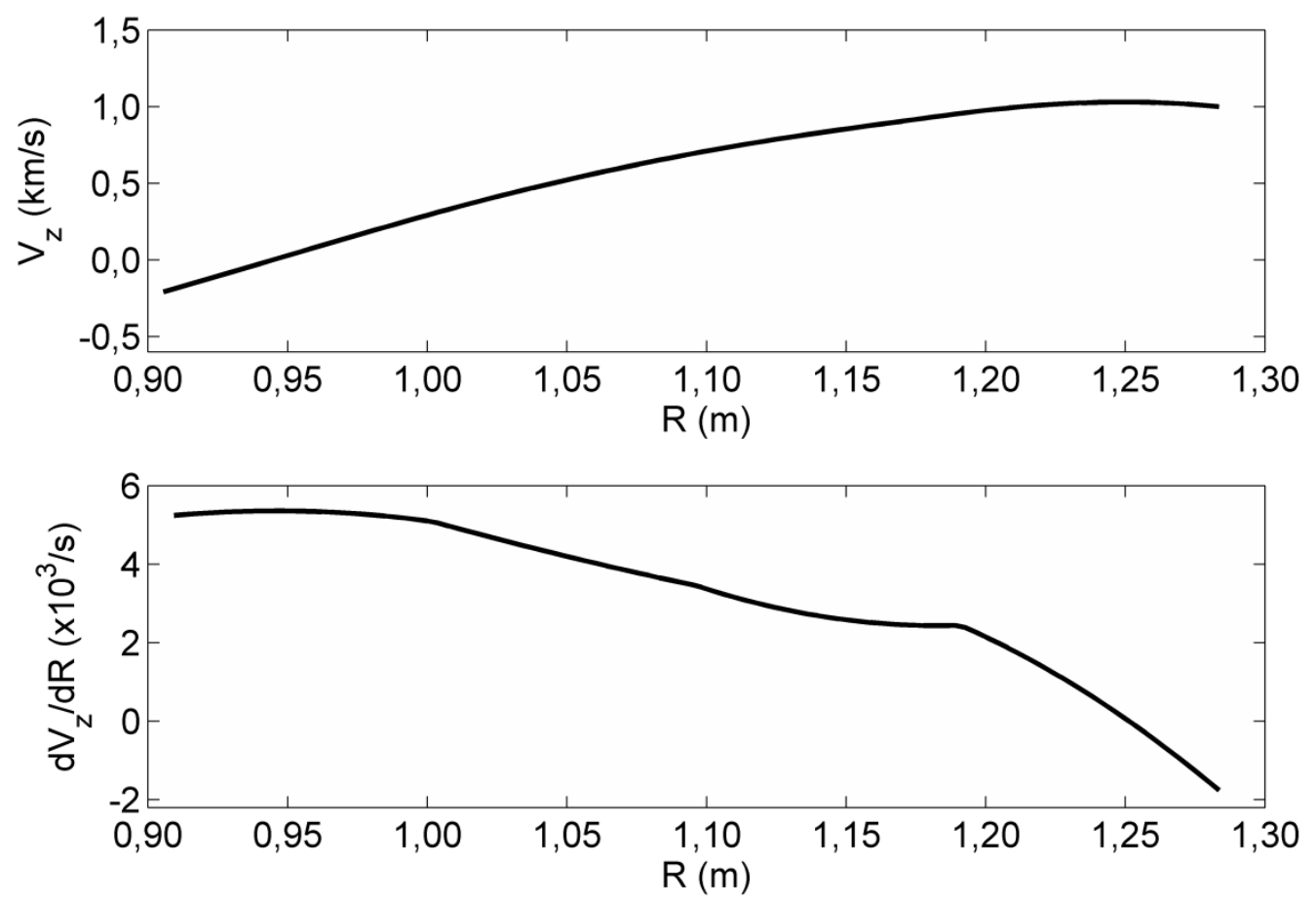

Figura 3.11: Exemplo de perfil radial de velocidade vertical de fluxo do plasma, $V_{z}$, e de perfil radial do cisalhamento (shear), $d V_{z} / d R$. 


\section{CARACTERIZAÇÃO DO PLASMA NÃO PERTURBADO NO TEXAS HELIMAK}

Neste capítulo serão apresentadas e discutidas as características do plasma e da sua turbulência no Texas Helimak. São apresentados resultados para o plasma não perturbado, com o potencial elétrico externo (bias) nulo, ou seja, as placas utilizadas para impor o bias estão aterradas e consequentemente tem potencial nulo.

Este capítulo apresenta resultados sobre o equilíbrio e turbulência. Sobre o equilíbrio, são analisadas as principais grandezas médias do plasma e sua dependência com as posições radial e vertical. Já sobre a turbulência, são apresentadas as características estatísticas e espectrais das flutuações do plasma. O espectro de densidade de potência em função da frequência e do número de onda foi utilizado para determinar a velocidade de fase média do espectro e sua dependência com a coordenada radial.

\subsection{Equilíbrio}

Na Figura 4.1 são apresentados os perfis radiais médios da corrente de saturação iônica (a), do potencial flutuante (b) e da temperatura eletrônica (c). No perfil de corrente de saturação, que é aproximadamente proporcional à densidade do plasma, a corrente de saturação apresenta um máximo na faixa $0,90 \mathrm{~m}<\mathrm{R}<1,10 \mathrm{~m}$, sendo $\mathrm{o}$ gradiente da corrente de saturação maior na região interna da máquina do que na região externa. Observa-se que o gradiente de $\mathrm{I}_{\text {sat }}$ é praticamente constante para $1,100 \mathrm{~m}<\mathrm{R}<$ $1,250 \mathrm{~m}$. Analisando o perfil de potencial flutuante percebe-se que este tem como principal característica um mínimo para $\mathrm{R} \cong 1,050 \mathrm{~m}$. Além disso, o potencial apresenta valores de mínimo não muito acentuados tanto na região mais interna da máquina, $\mathrm{R}<$ $0,90 \mathrm{~m}$, quanto na região mais externa $\mathrm{R}>1,20 \mathrm{~m}$. No perfil de temperatura do plasma, o que se observa é que a temperatura do plasma sobe gradativamente com a posição radial até atingir um patamar para aproximadamente1,0 $\mathrm{m}<\mathrm{R}<1,15 \mathrm{~m}$, depois do qual a temperatura volta a cair com o raio até $\mathrm{R} \cong 1,22 \mathrm{~m}$. 

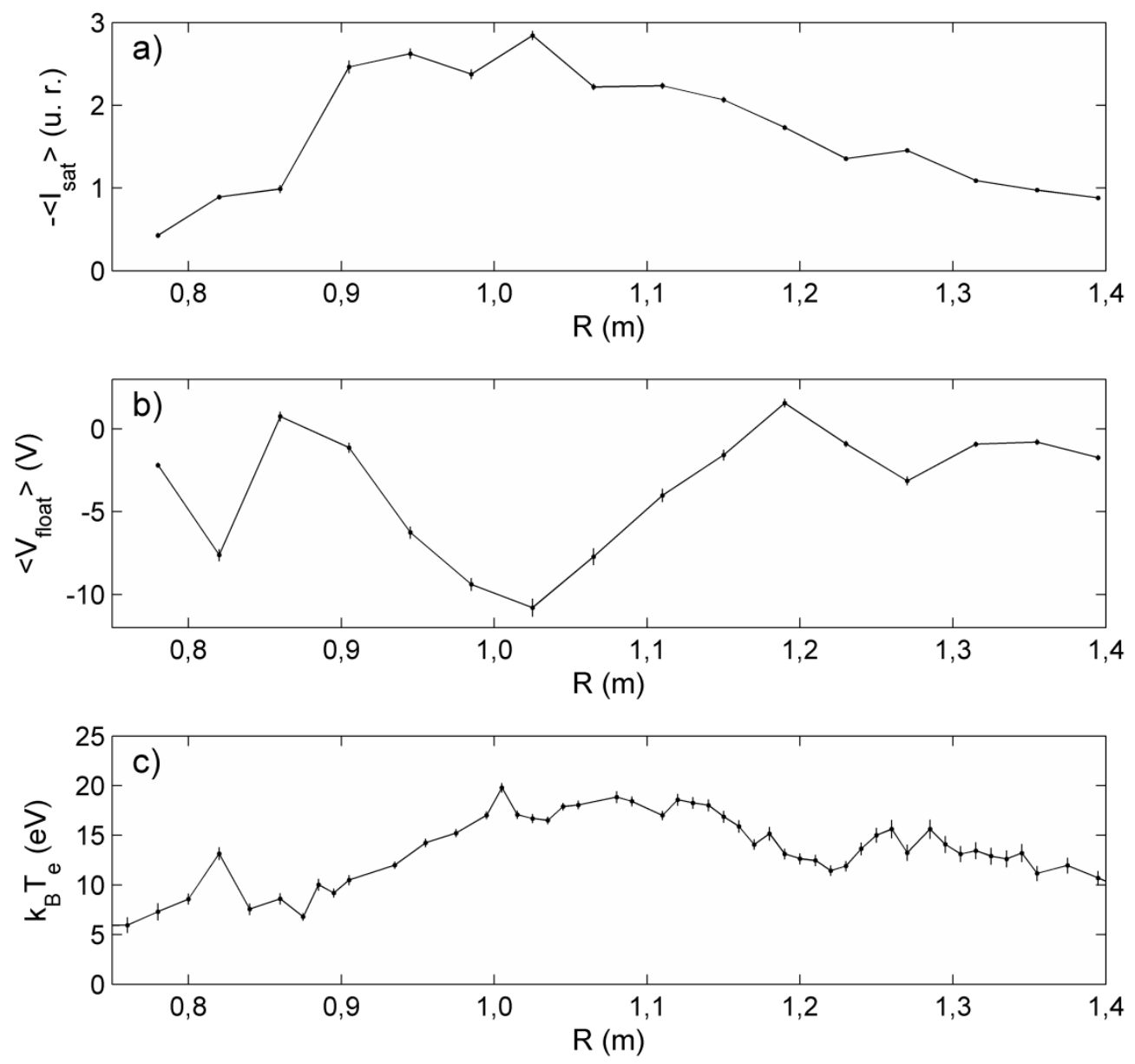

Figura 4.1: Perfis radiais de corrente de saturação média (a), potencial flutuante médio (b) e temperatura média (c).

No Texas Helimak o equilíbrio do plasma é praticamente unidimensional, pois suas características básicas variam apenas com a coordenada radial. Para verificar isso, na Figura 4.2 esta representada a corrente de saturação iônica média com função do raio e da posição vertical, z. Nesta figura o valor da corrente de saturação esta representado na escala de cores. Note-se que, a menos de pequenas variações, o perfil radial da corrente de saturação não depende da posição vertical da sonda. Além disso, devido à simetria das linhas de campo magnético, as grandezas de equilíbrio não dependem da seção poloidal do plasma. 


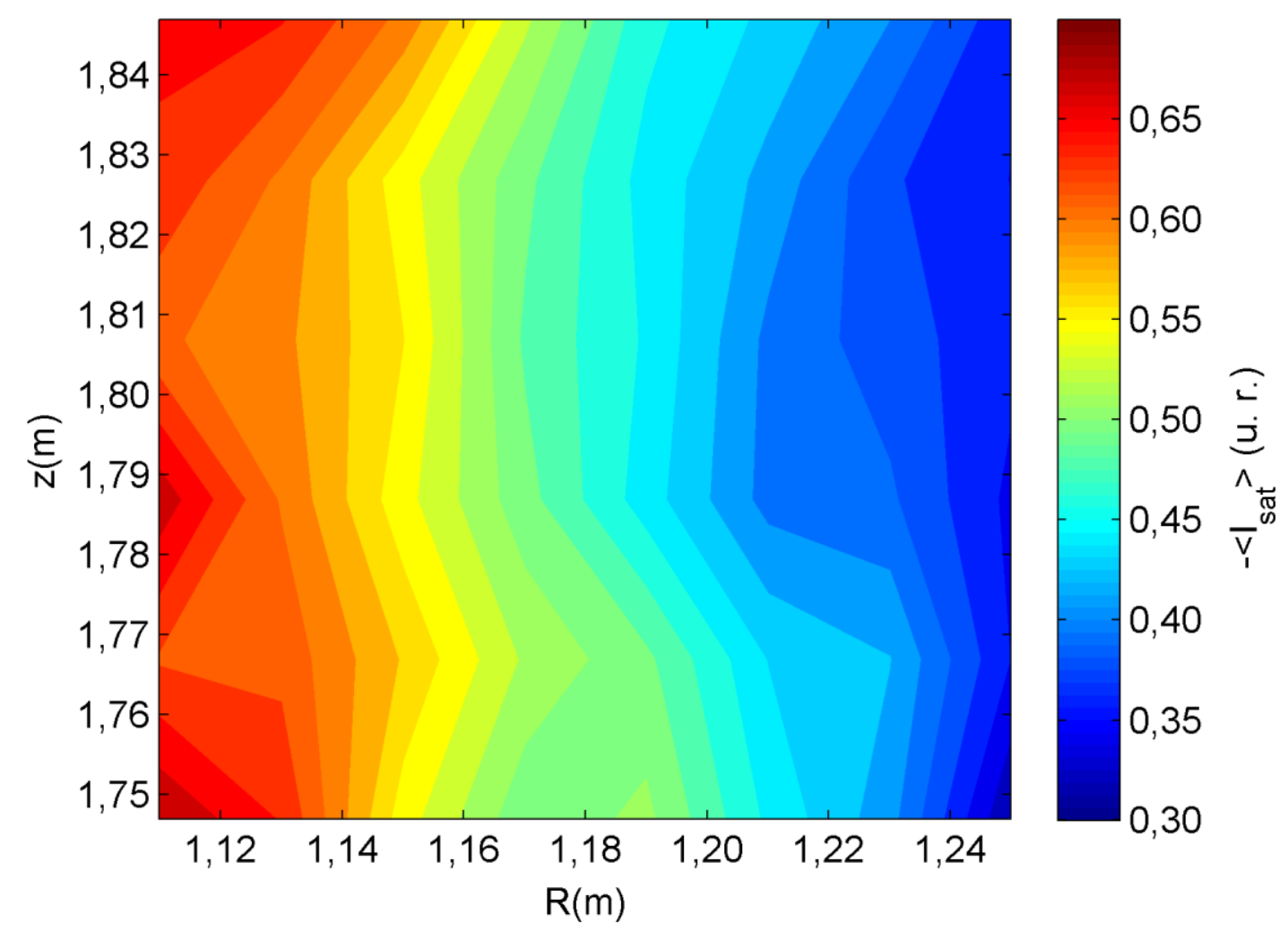

Figura 4.2: Valores médios de corrente de saturação iônica em função do raio (R) e da posição vertical (z). Nesta figura apenas está mostrada a faixa radial de maior interesse: $1,10 \mathrm{~m}<\mathrm{R}<1,25 \mathrm{~m}$.

\subsection{Turbulência: caracterização estatística}

A principal medida estatística da turbulência, utilizando sondas de Langmuir, é o nível de turbulência, que para corrente de saturação iônica é definido como $\sigma_{I_{s a t}} /\left|\overline{I_{\text {sat }}}\right| \mathrm{e}$ para o potencial flutuante é definido como $\sigma_{V_{\text {float }}} / k_{B} \bar{T}_{e}$. Considerando a flutuação de temperatura como relativamente constante, as seguintes relações podem ser tomadas como boas aproximações:

$$
\begin{aligned}
\frac{\sigma_{\varphi}}{k_{B} \overline{T_{e}}} \cong \frac{\sigma_{V_{\text {float }}}}{k_{B} \overline{T_{e}}}, \\
\frac{\sigma_{n}}{\bar{n}} \cong \frac{\sigma_{I_{\text {sat }}}}{\left|\overline{I_{\text {sat }}}\right|},
\end{aligned}
$$


sendo $\sigma_{n}$ o desvio padrão das flutuações de densidade, $\bar{n}$ a média temporal da densidade, $\sigma_{\varphi}$ o desvio padrão das flutuações do potencial do plasma, $\bar{T}_{e}$ a média temporal da temperatura eletrônica e $k_{B}$ a constante de Boltzmann.

Assumindo as relação 4.1 e 4.2 como válidas, os perfis radiais do nível de turbulência da densidade e do potencial do plasma foram calculados e o resultado é apresentado na Figura 4.3. Para este cálculo foram analisados sinais de $10 \mathrm{~s}$ de duração, divididos em 100 janelas de 100 ms e 10 mil pontos cada. Na Figura 4.3 o nível de turbulência da densidade está representado em azul e do potencial do plasma em vermelho e as linhas desenhadas apenas representam a tendência geral dos dados, sem considerar nenhum modelo em particular.

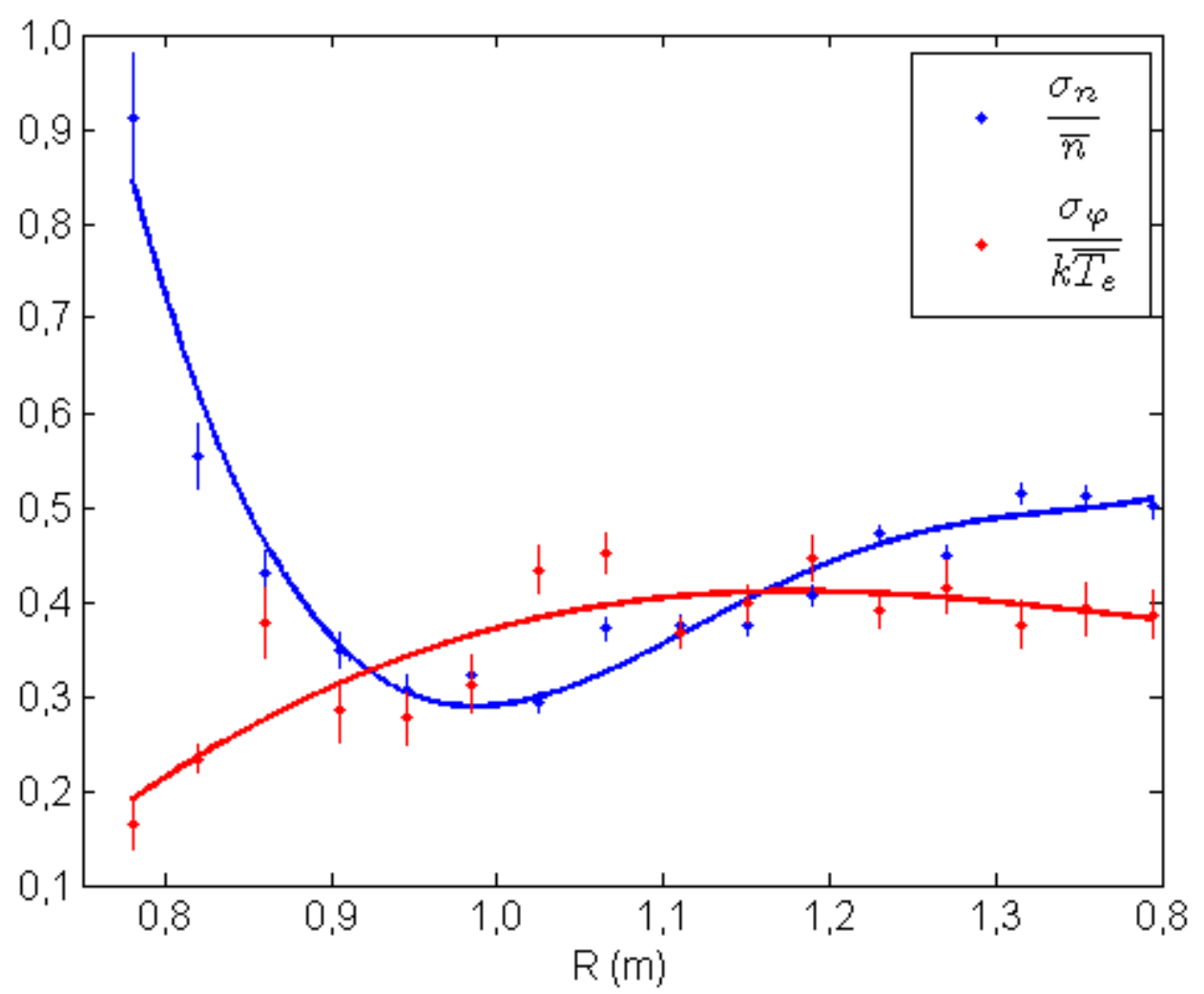

Figura 4.3: Perfis radiais aproximados de nível de turbulência da densidade (azul) e do potencial do plasma (vermelho), as curvas representam a tendência dos dados. Nota-se que as duas curvas estão mais próxima na faixa radial $1,10 \mathrm{~m}<\mathrm{R}<1,25 \mathrm{~m}$, mostrando que nesta faixa a relação de adiabaticidade pode ser considerada satisfeita.

Na Figura 4.3 nota-se que os níveis de turbulência da densidade $\left(\sigma_{n} / \bar{n}\right)$ e do potencial do plasma $\left(\sigma_{\varphi} / k_{B} \bar{T}_{e}\right)$ apresentam valores próximos. Isso pode significar que, para certos valores de raio, especialmente na faixa $0,90 \mathrm{~m}<\mathrm{R}<1,25 \mathrm{~m}$, o plasma do Texas Helimak aproximadamente satisfaz a condição de adiabaticidade, dada pela equação 4.3: 


$$
\frac{\sigma_{n}}{\bar{n}} \cong \frac{\sigma_{\varphi}}{k_{B} \overline{T_{e}}}
$$

\subsection{Turbulência: caracterização espectral}

\subsubsection{Escolha da faixa radial de análise}

Para a análise espectral é importante a presença de sondas distribuídas verticalmente para que seja possível estudar e caracterizar ondas ou modos que se propagem na direção vertical. Como o número de ADCs disponíveis é limitado, faz-se necessário escolher uma faixa radial para concentrar as sondas cujos sinais serão adquiridos e analisados. Neste trabalho a faixa escolhida para a análise foi: $1,10 \mathrm{~m}<\mathrm{R}$ $<$ 1,25 m. A escolha desta faixa ocorre por diversas razões, sendo as principais consideradas as seguir.

Do ponto de vista experimental, as sondas de Langmuir são montadas em placas localizadas no fundo e no topo do vaso do Helimak, como vista na Figura 2.4. Dessa forma, o arranjo mais simples é escolher um conjunto de sondas em uma única placa, uma vez que sondas localizadas próximas à separação entre as placas podem produzir medidas incorretas devido à possibilidade de uma das placas produzir um efeito de "sombra" sobre as outras, bloqueando parcialmente o plasma e causando erros nas medidas. Na faixa radial escolhida, $1,10 \mathrm{~m}<\mathrm{R}<1,25 \mathrm{~m}$, todas as sondas ficam localizadas na terceira placa. Além disso no controle do plasma, conseguido através da imposição de potencial elétrico externo, bias, é sempre utilizado a segunda placa de cada conjunto, localizadas entre $\mathrm{R}=0,863 \mathrm{~m}$ e $\mathrm{R}=1,075 \mathrm{~m}$ e marcadas em amarelo na Figura 2.1, para impor o bias. Sendo assim, a região radial mais adequada para estudar os efeitos do controle no plasma é a região imediatamente ao lado da placa com o bias pois é nela que se espera um maior gradiente de potencial e, consequentemente, maior campo elétrico.

Do ponto de vista das características do plasma, a faixa $1,10 \mathrm{~m}<\mathrm{R}<1,25 \mathrm{~m}$ também é a mais adequada pois o gradiente da densidade nesta faixa é praticamente constante, vide Figura 4.1, o que facilita a análise de efeitos que dependem, além de outros fatores, do gradiente da densidade. Além disso, como visto na Figura 4.3, é nesta 
faixa de valores radiais que a aproximação de plasma adiabático torna-se mais aceitável. Essas características facilitam a elaboração de previsões teóricas que podem ser verificadas no Texas Helimak.

A distribuição de sondas utilizadas nesta análise espectral é vista na Figura 4.4. Note que as sondas cujos sinais foram adquiridos estão dispostas principalmente na faixa $1,10 \mathrm{~m}<\mathrm{R}<1,25 \mathrm{~m}$ e em posições verticais entre $\mathrm{z}=1,747 \mathrm{~m}$ e $\mathrm{z}=1,847 \mathrm{~m}$.

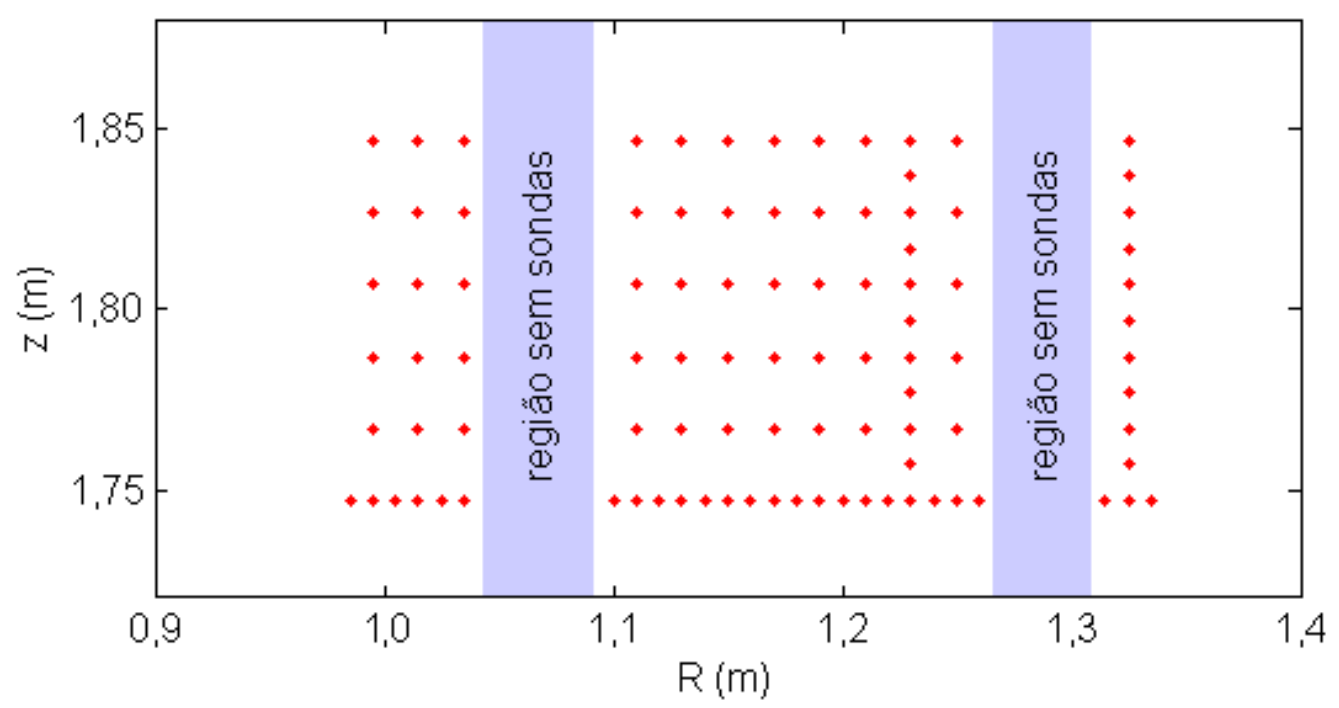

Figura 4.4: Distribuição de sondas utilizada para coletar os dados da análise espectral.

\subsubsection{Caracterização espectral}

Nos sinais aqui analisados, originalmente obtidos em descargas de $22 \mathrm{~s}$ de duração, foram analisados intervalos de 9,7 s nos quais o plasma é estacionário. Como foi utilizado o ADC de $500 \mathrm{kHz}$ de taxa de amostragem as séries analisadas têm aproximadamente $4,9 \times 10^{6}$ pontos. Para a análise espectral foi utilizada a transformada de Fourier janelada, dividindo cada série de dados em 194 séries com 25 x $10^{3}$ pontos cada.

A medida espectral mais direta é o espectro de potência. A Figura 4.5 mostra o espectro de potência para quatro posições radiais diferentes. Nesta figura nota-se que a potência do sinal diminui a medida que a coordenada radial aumenta e que a potência espectral está distribuída em uma banda larga, característica típica da turbulência. Observa-se também que para valores radiais menores existe a presença de um pico de baixa frequência, $f \cong 100 \mathrm{~Hz}$. 

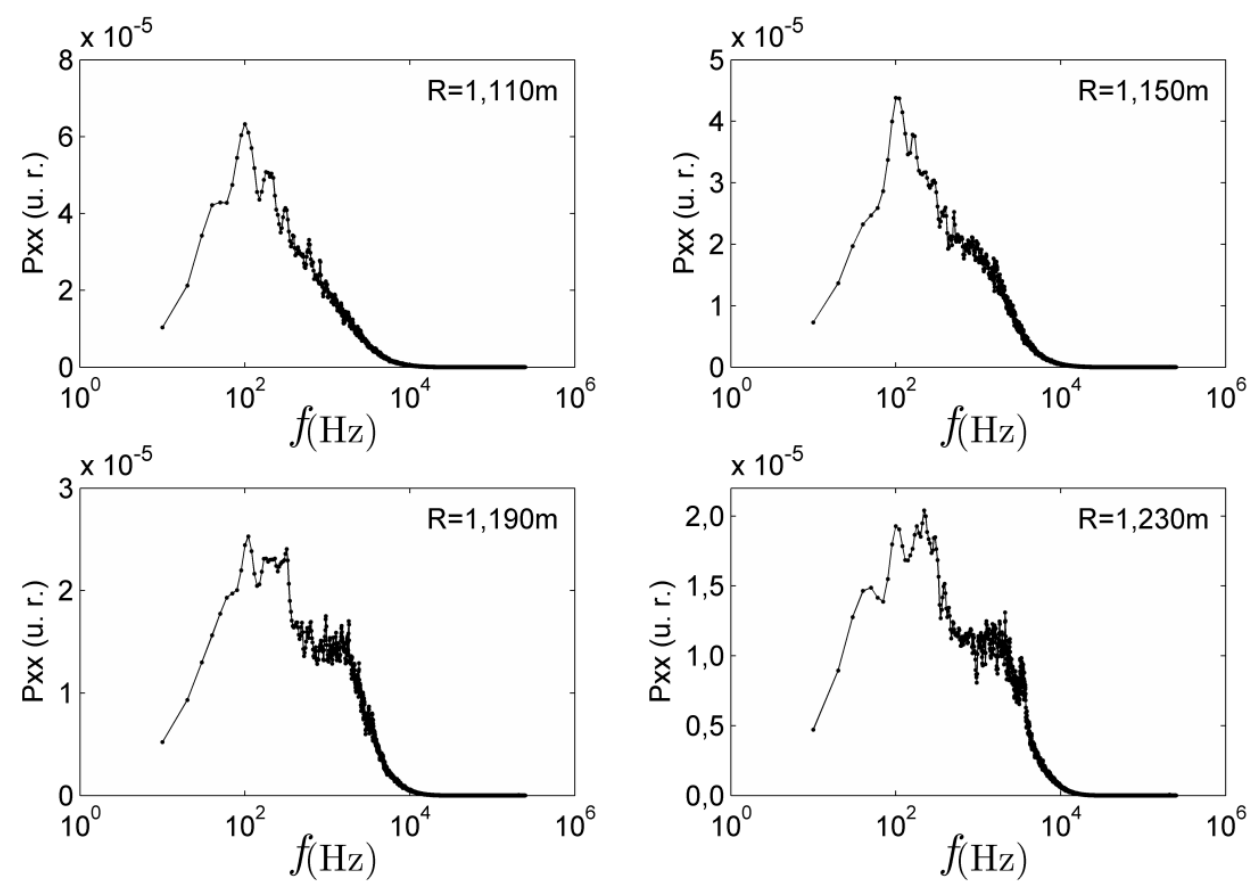

Figura 4.5: Espectro de potência, Pxx, de corrente de saturação iônica para quatro sondas posicionadas em quatro raios distintos e mesma altura $(\mathrm{z}=1,747 \mathrm{~m})$.

A fim de verificar se o espectro de potência conserva suas características, ao longo dos quase dez segundos em que a análise espectral é realizada, foram construídos espectrogramas em função do tempo e da frequência, em que a potência espectral calculada para cada janela de tempo está representada na escala de cinza. Os quatro espectrogramas de corrente de saturação iônica calculados para as mesmas sondas e mesmo disparo utilizados nos espectros de potência da Figura 4.5 são vistos na Figura 4.6. Nota-se a partir desta última figura que, ao longo dos dez segundos de disparo, os espectros de potência conservam suas características tanto em relação a maior concentração de potência para frequências em torno de $100 \mathrm{~Hz}$ como em relação a razão com que o espectro de potência cai para frequências altas. 

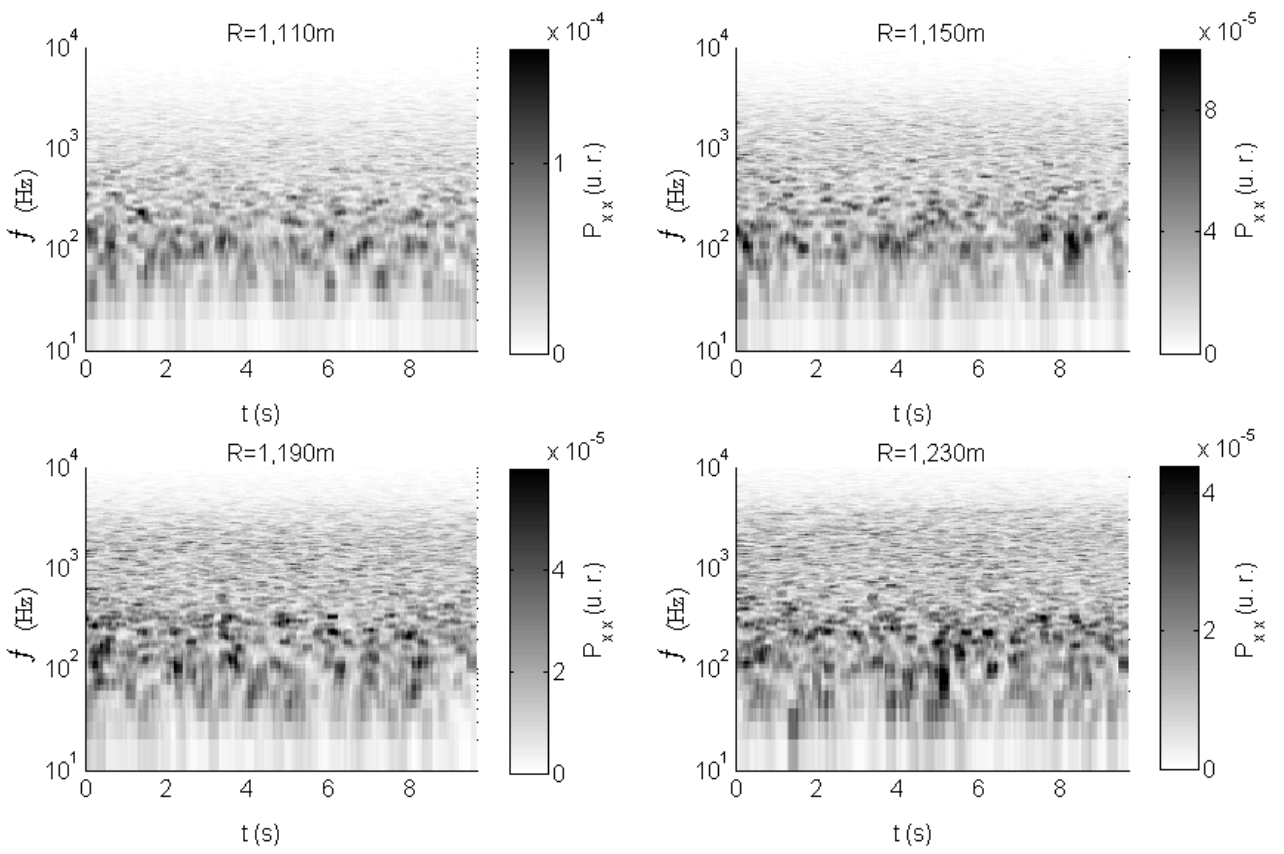

Figura 4.6: Espectrogramas de potência, Pxx, de corrente de saturação iônica para quatro sondas posicionadas em quatro raios distintos e mesma altura $(\mathrm{z}=1,747 \mathrm{~m})$.

Uma vez verificada a estabilidade das características do espectro de potência, este foi calculado para todas as posições radiais disponíveis dentro da faixa $1,10 \mathrm{~m}<\mathrm{R}$ $<1,25 \mathrm{~m}$. O resultado é mostrado na Figura 4.7, na forma de um gráfico em que a potência espectral $(P x x)$ é representada na cor do gráfico.

Analisando o espectro da Figura 4.7, nota-se primeiramente a presença de dois picos com frequências $f \cong 100 \mathrm{~Hz}$ e $f \cong 200 \mathrm{~Hz}$, com potência maior para posições radiais mais internas. Estes picos podem ser mais bem observados na Figura 4.5. Observa-se também que, quase que a totalidade da energia do espectro está concentrada em $f \leq 10 \mathrm{kHz}$. 


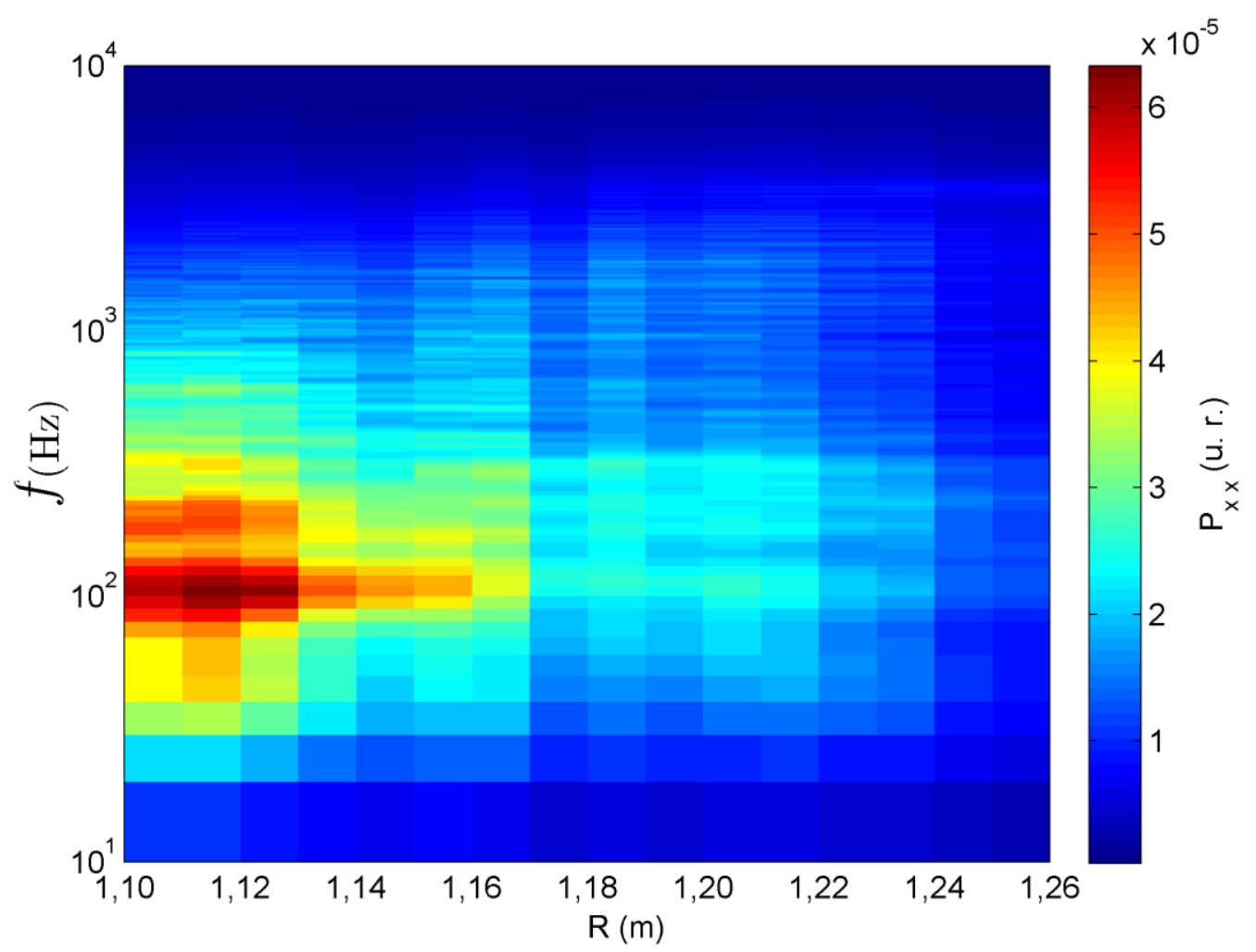

Figura 4.7: Espectro de potência, Pxx, em função da posição radial.

Além da potência espectral, identificar a velocidade de fase da turbulência é importante para entender as características espectrais do plasma. Para isso a Figura 4.8(a) mostra os espectros de número de onda, $k(f)$, em função da coordenada radial, calculados para pares de sondas com $\mathrm{z}=1,747 \mathrm{~m} \mathrm{e} 1,767 \mathrm{~m}(\Delta \mathrm{z}=2 \mathrm{~cm})$. Nesta figura nota-se que na região de maior interesse, $1,10 \mathrm{~m}<\mathrm{R}<1,25 \mathrm{~m}$, o número de onda $k(f)$ é sempre negativo, aumentando de valor (em módulo) para frequências mais altas e raios maiores. Na Figura 4.8 também são mostrados dois espectros de $k(f)$ para dois valores de raio, $\mathrm{R}=1,110 \mathrm{~m}$ (b) e $\mathrm{R}=1,170 \mathrm{~m}$ (c) ilustrando a relação aproximadamente linear entre $k$ e $f$, o que indica uma velocidade de fase vertical única para todas as frequências do plasma. 

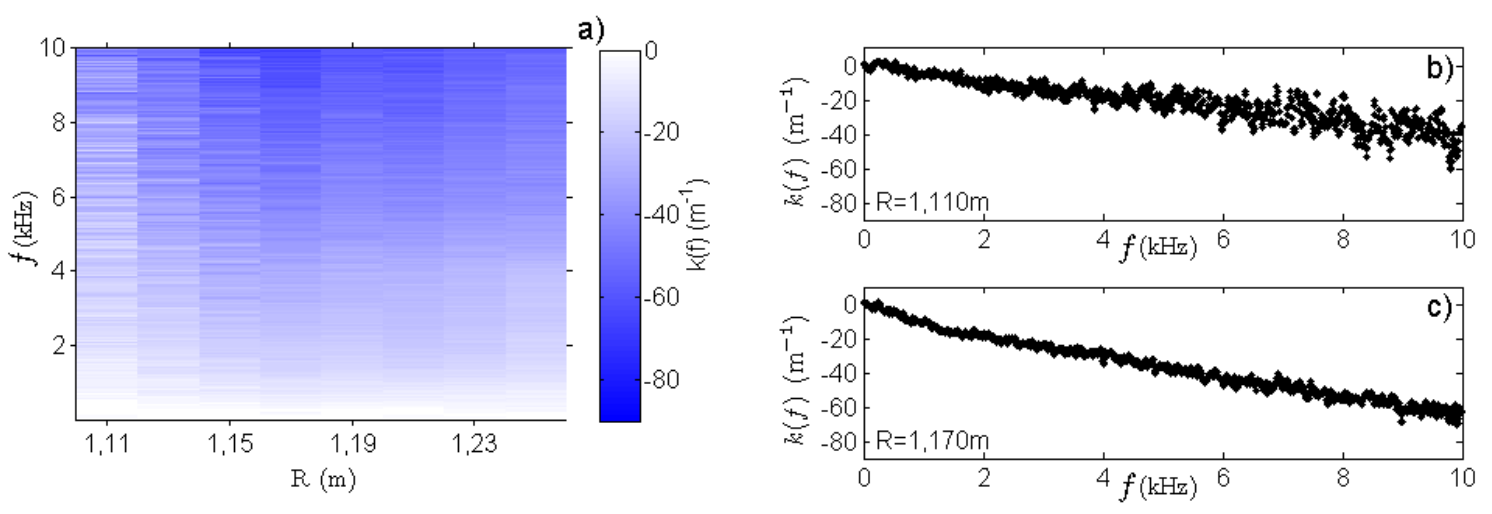

Figura 4.8: Espectros de $k(f)$ em função do raio no qual o valor de $k(f)$ esta representado pela escala de cor (a) e dois espectros de $k(f)$ para dois valores de raio, $\mathrm{R}=1,110 \mathrm{~m}$ (b) e $\mathrm{R}=1,170 \mathrm{~m}$ (c), ilustrando a relação linear entre o número de onda e a frequência. Esses espectros mostram que o sentido da velocidade de fase não se inverte na região radial $1,10 \mathrm{~m}<\mathrm{R}<1,25 \mathrm{~m}$.

Como a relação de $k$ com $f$ é aproximadamente linear, é possível estimar o valor médio da velocidade de fase das ondas, viajando no plasma, através do espectro de $S(k, f)$, como mostrado no capítulo 3 - equação 3.10. O espectro de $S(k, f)$ para bias nulo e $\mathrm{R}=1,170 \mathrm{~m}$ é visto na Figura 4.9. Nesta figura a velocidade de fase estimada através do espectro de $S(k, f)$ é ilustrada pela reta em preto que foi acrescentada à figura. Ao comparar a reta com o espectro, observa-se que a velocidade de fase é uma boa aproximação para a região de maior potência do espectro $(f \leq 2 \mathrm{kHz})$.

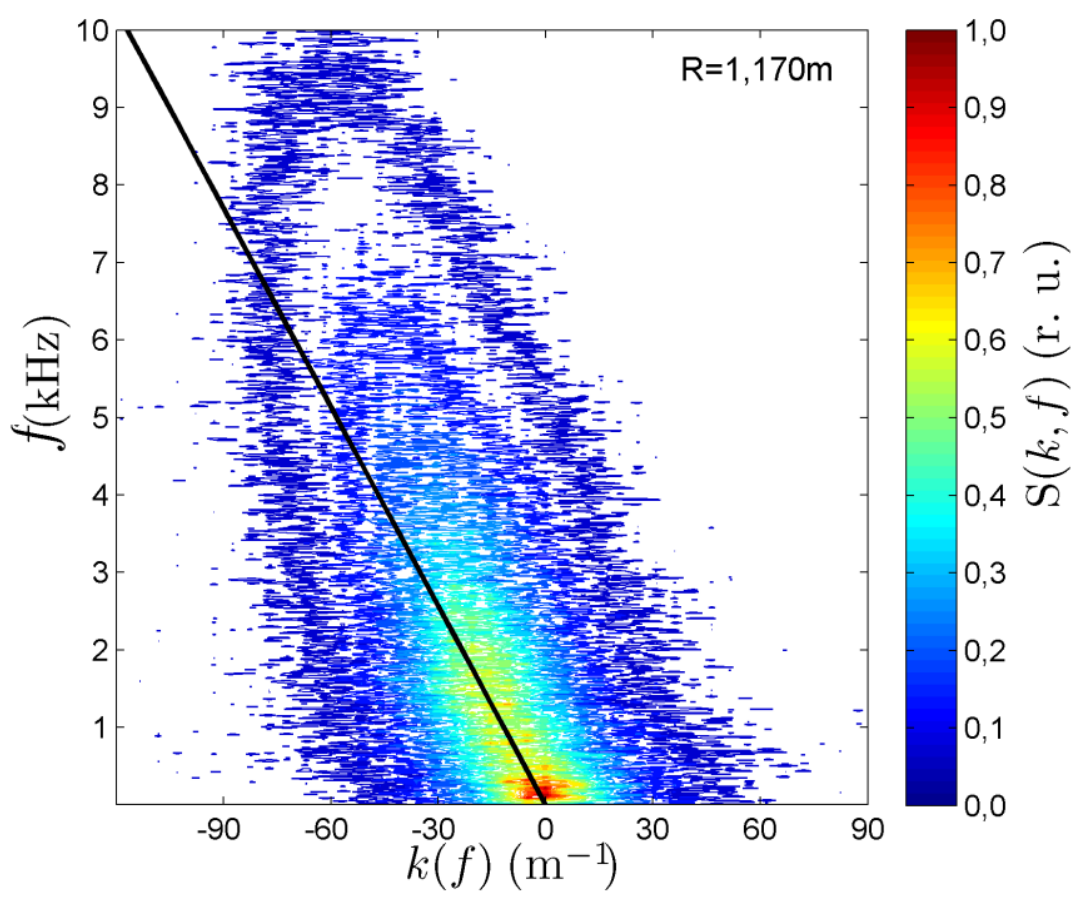

Figura 4.9: Espectro de $S(k, f)$ para raio $\mathrm{R}=1,170 \mathrm{~m}$. A reta em preto ilustra a velocidade de fase média estimada. Nota-se que a velocidade de fase média estimada é uma boa aproximação para a região de maior potência do espectro $(f \leq 2 \mathrm{kHz})$. 
A Figura 4.10 mostra o perfil radial da velocidade de fase estimado através do espectro de $S(k, f)$ para pares de sondas posicionadas em $z_{1}=1,747 \mathrm{~m} \mathrm{e} z_{2}=1,767 \mathrm{~m}$ $(\Delta \mathrm{z}=2 \mathrm{~cm})$. Nesta figura fica claro a que a velocidade de fase estimada aumenta (em módulo) com a coordenada radial.

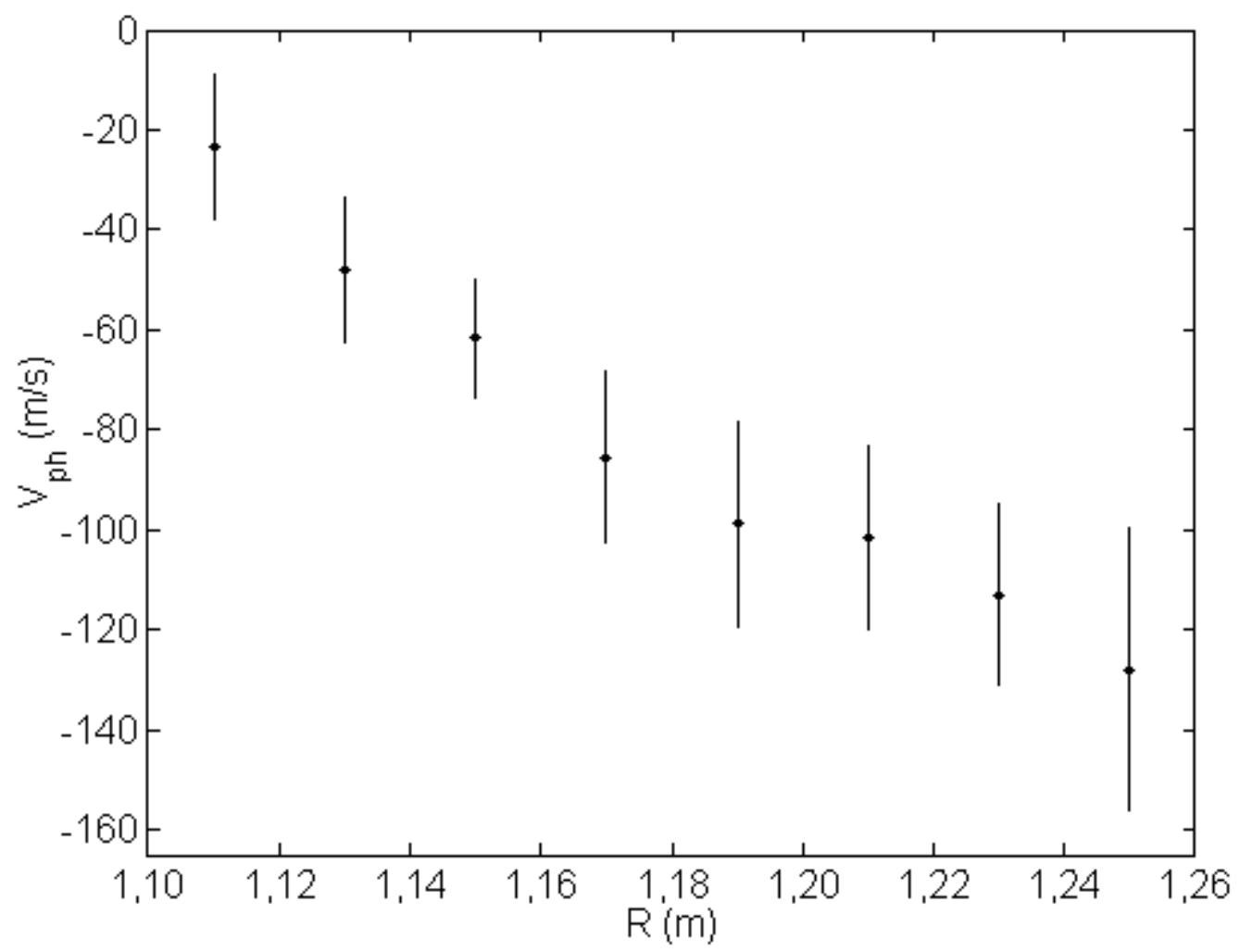

Figura 4.10: Velocidade de fase $\left(\mathrm{V}_{\mathrm{ph}}\right)$ estimada através do espectro de $S(k, f)$ em função da posição radial.

\subsection{Considerações finais acerca das características do plasma não perturbado}

Considerando o que foi mostrado neste capítulo, podemos concluir em primeiro lugar que o plasma no Texas Helimak apresenta um comportamento muito diversificado. De um modo geral, todos os parâmetros médios do plasma dependem da coordenada radial. Assim a corrente de saturação média, proporcional à densidade média, apresenta um máximo no seu perfil radial em torno de $\mathrm{R}=1,00 \mathrm{~m}$ e um gradiente negativo praticamente constante no intervalo $1,10 \mathrm{~m}<\mathrm{R}<1,25 \mathrm{~m}$. A exceção é a temperatura eletrônica média que apresenta variações relativamente pequenas no 
intervalo radial $1,00 \mathrm{~m}<\mathrm{R}<1,30 \mathrm{~m}$. Em relação a coordenada vertical, as características do plasma são praticamente constantes ao longo dos $10 \mathrm{~cm}$ em que as sondas estão verticalmente distribuídas.

A análise estatística das flutuações de corrente de saturação iônica e de potencial flutuante mostrou que a condição de adiabaticidade pode ser considerada uma boa aproximação na faixa radial que vai de $\mathrm{R}=0,90 \mathrm{~m}$ até $\mathrm{R}=1,25 \mathrm{~m}$.

A análise espectral dos sinais de corrente de saturação iônica revelou que as características do espectro de potência se preservam ao longo do tempo, mas se alteram de acordo com a posição radial. Para raios menores observa-se a presença de um modo de baixa frequência, cuja amplitude diminui em posições radiais maiores. O número de onda médio na direção vertical mostra uma dependência linear com a frequência, o que indica que as ondas de todas as frequências se propagam no plasma com a mesma velocidade de fase. Por fim mostrou-se que a velocidade de fase aumenta, em módulo, para posições radiais maiores.

O efeito do controle (bias $\neq 0$ ) sobre essas características do plasma e da turbulência será tratado no capítulo seguinte. 


\section{CONTROLE ATRAVÉS DO BIAS EXTERNO}

Este capítulo tem como objetivo apresentar o efeito do potencial elétrico externo ao plasma, chamado de bias, nas principais características do plasma do Texas Helimak. Em todas as experiências analisadas neste trabalho o bias é imposto em quatro placas localizadas entre $\mathrm{R}=0,863 \mathrm{~m}$ e $\mathrm{R}=1,075 \mathrm{~m}$, no fundo, no topo e nos dois lados da máquina.

Este capítulo foi dividido em seções que tratam dos parâmetros do plasma em equilíbrio e da turbulência em separado. Serão apresentados resultados para o plasma perturbado por valores de bias positivos e negativos. Esses resultados são comparados aos obtidos sem a perturbação (com o potencial elétrico externo nulo).

\subsection{Plasma em equilíbrio}

Na Figura 5.1 estão apresentados os perfis radiais da média temporal de corrente de saturação iônica $\left(\mathrm{I}_{\text {sat }}\right)$ para cinco valores distintos de bias $(-20 \mathrm{~V},-8 \mathrm{~V}, 0,+8 \mathrm{~V}$ e $+20 \mathrm{~V}$ ). Observa-se que o gradiente de $\mathrm{I}_{\text {sat }}$ é praticamente constante para $1,10 \mathrm{~m}<\mathrm{R}<$ 1,25 m e que esse gradiente praticamente não varia em função do bias aplicado. Essas duas características tornam essa região radial interessante para análise. Por outro lado, na Figura 5.1 percebe-se que, para valores negativos de bias, o valor máximo da corrente de saturação iônica esta na região mais interna da máquina $(\mathrm{R}<1 \mathrm{~m})$. A Figura 5.2 mostra o panorama geral da média da corrente de saturação iônica como função do raio e do bias. Nesta figura fica claro que o efeito do bias na corrente de saturação iônica e, consequentemente, na densidade, é pequeno pois não são observados grandes deslocamentos da região em vermelho na qual estão os maiores valores de densidade. 

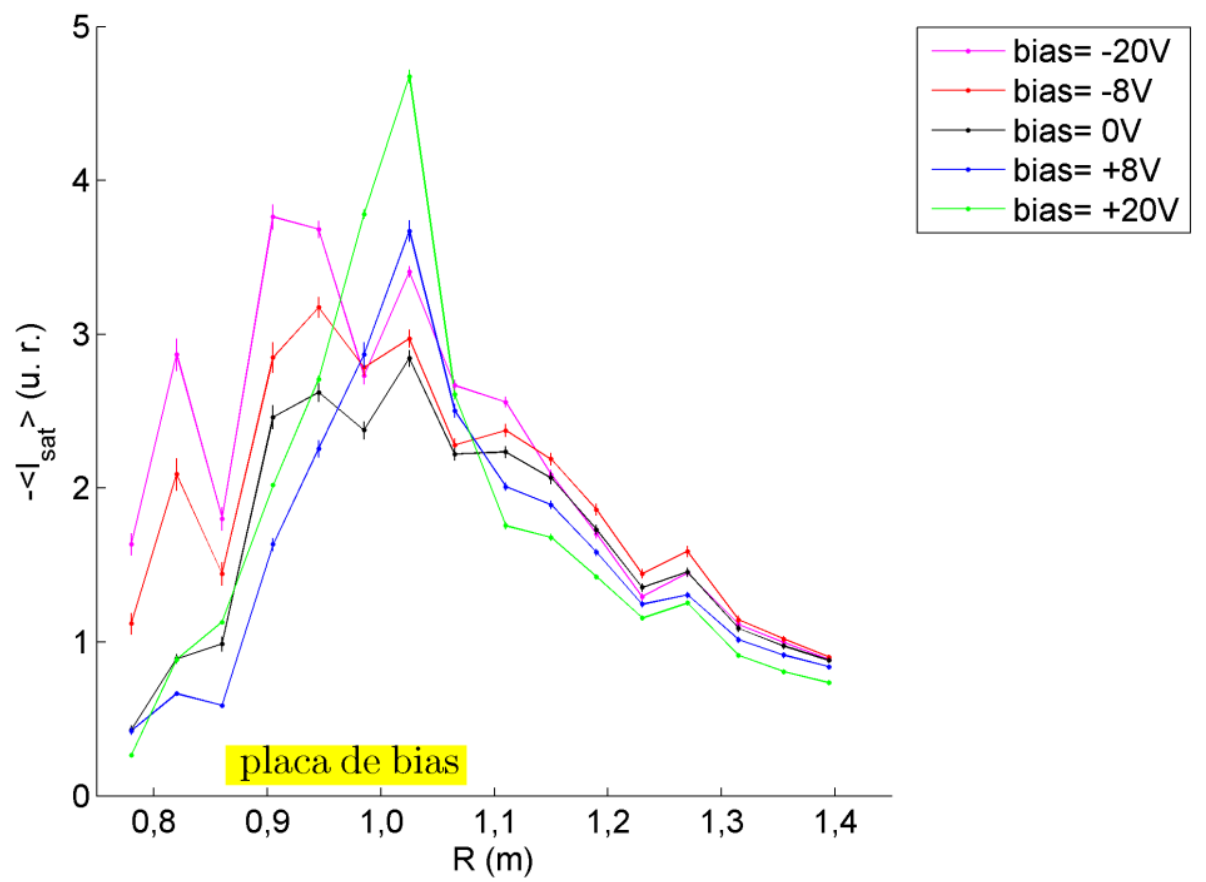

Figura 5.1: Perfis radiais médios de corrente de saturação iônica para cinco valores distintos de bias ($20 \mathrm{~V},-8 \mathrm{~V}, 0,+8 \mathrm{~V} \mathrm{e}+20 \mathrm{~V})$. Todas as medidas foram feitas com sondas posicionadas na mesma posição vertical da máquina $(\mathrm{z}=1,747 \mathrm{~m})$.

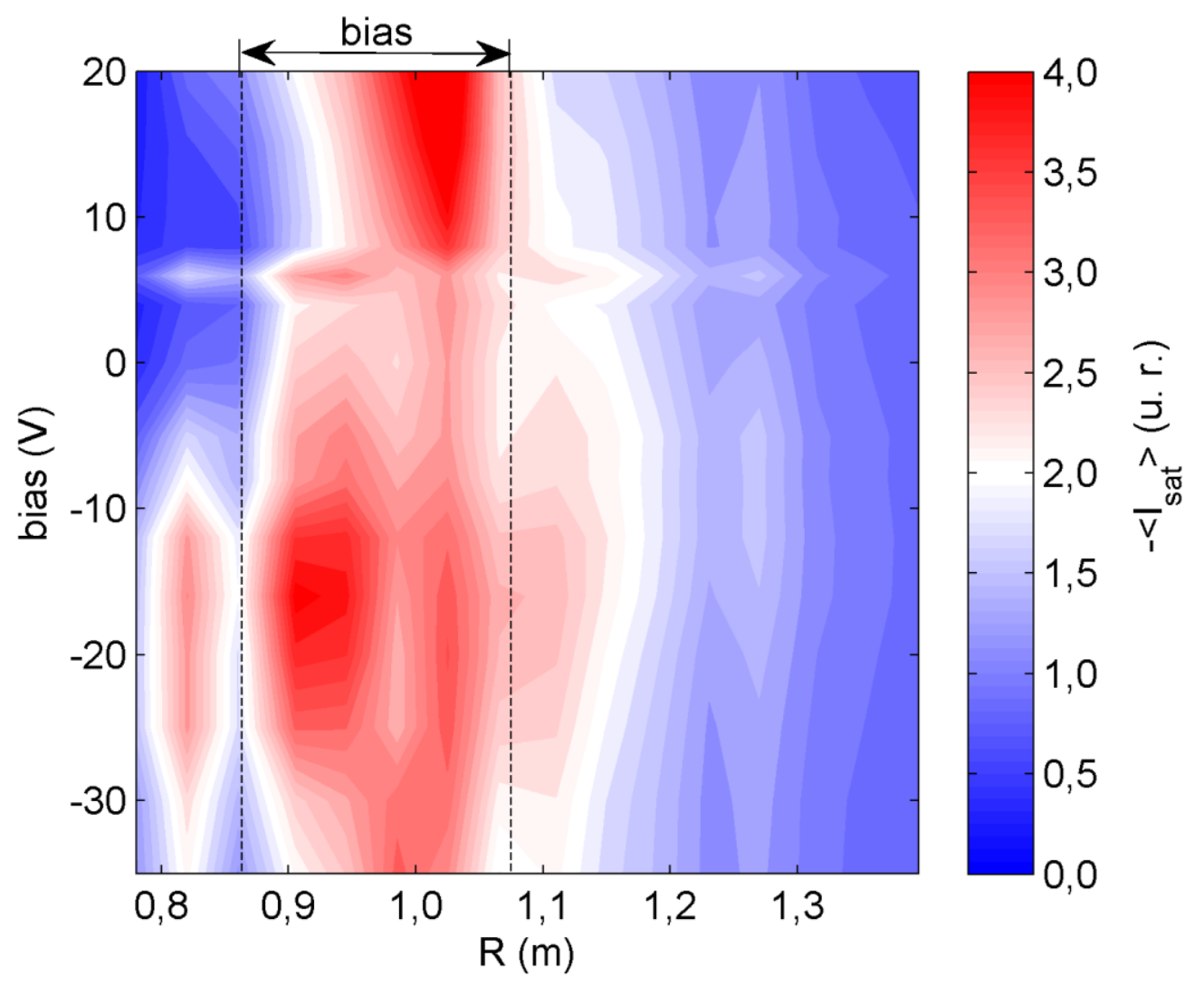

Figura 5.2: Corrente de saturação iônica média como função da posição radial da sonda e do valor de bias aplicado. As linhas pontilhas marcam a região radial onde esta localizada a placa de bias. Todas as medidas foram feitas com sondas posicionadas na mesma posição vertical da máquina $(\mathrm{z}=1,747 \mathrm{~m})$. 
Como comentado, o bias tem pouca influência nos perfis radiais de corrente de saturação iônica. Consequentemente, os perfis de densidade do plasma (obtidos dos perfis da corrente de saturação) devem sofrer pouca influência do bias. Por outro lado, o potencial do plasma deve ser intensamente afetado pelo bias. Isso pode ser avaliado pelos perfis de potencial flutuante $\left(\mathrm{V}_{\text {float }}\right)$, vistos na Figura 5.3, que mostram uma grande variação dos valores de $\mathrm{V}_{\text {float }}$ na região radial em que se encontram as placas de bias. De um modo geral pode-se dizer que o potencial flutuante do plasma na região da placa de bias acompanha o valor do bias, tornando-se muito negativo (positivo) quando o bias é negativo (positivo). Tal comportamento fica evidente ao compararmos as curvas para bias $=-20 \mathrm{~V}$ (curva em magenta) bias $=+20 \mathrm{~V}$ (curva verde). A Figura 5.4 mostra o panorama geral do potencial médio flutuante em função do raio e do bias.

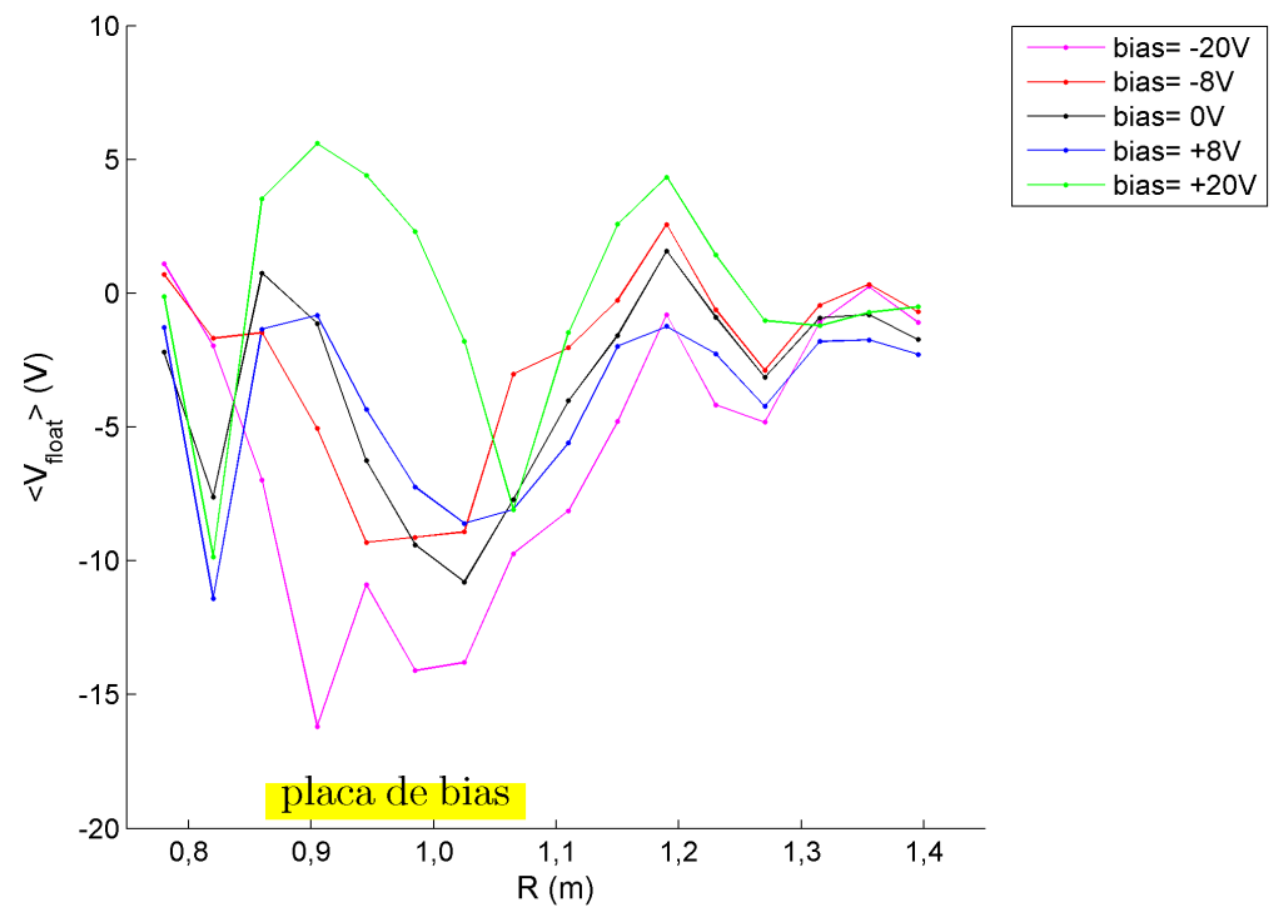

Figura 5.3: Perfis radiais médios de potencial flutuante para cinco valores distintos de bias (-20 V, $-8 \mathrm{~V}$, $0,+8 \mathrm{~V} \mathrm{e}+20 \mathrm{~V})$. Todas as medidas foram feitas com sondas posicionadas na mesma posição vertical $\mathrm{z}=1,747 \mathrm{~m}$. 


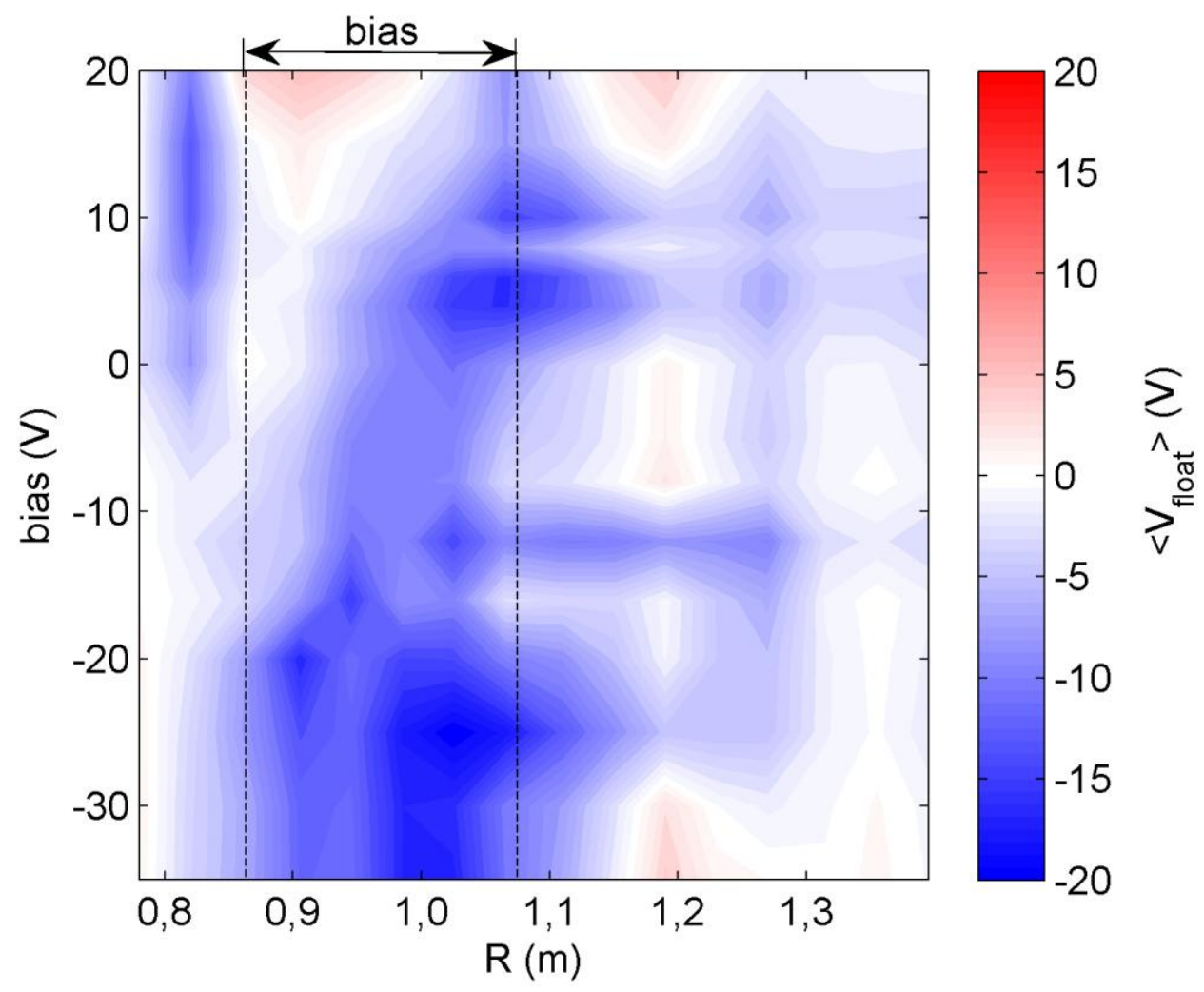

Figura 5.4: Potencial flutuante médio como função da posição radial da sonda e do valor de bias aplicado. As linhas pontilhas marcam a região radial onde esta localizada a placa de bias. Todas as medidas foram feitas com sondas posicionadas na mesma posição vertical $\mathrm{z}=1,747 \mathrm{~m}$.

A influência do bias no perfil radial de densidade também está ligada ao comportamento da temperatura eletrônica do plasma como função do bias. A Figura 5.5 mostras os perfis de temperatura eletrônica do plasma, estimados a partir da medida das curvas IxV das sondas, para cinco valores de bias $(-20 \mathrm{~V},-8 \mathrm{~V}, 0,+8 \mathrm{~V}$ e $+20 \mathrm{~V})$. A influência do bias nos perfis de temperatura é mais importante na região radial em que a temperatura apresenta um patamar, aproximadamente $1,00 \mathrm{~m}<\mathrm{R}<1,15 \mathrm{~m}$, sendo que a temperatura no patamar tende a diminuir para valores negativos de bias e a aumentar para valores positivos de bias. Para valores negativos de bias observa-se também um deslocamento do máximo da temperatura para raios mais internos. Tal comportamento fica claro ao observarmos a Figura 5.6, que mostra o comportamento geral da temperatura eletrônica média com função do raio e do bias. 


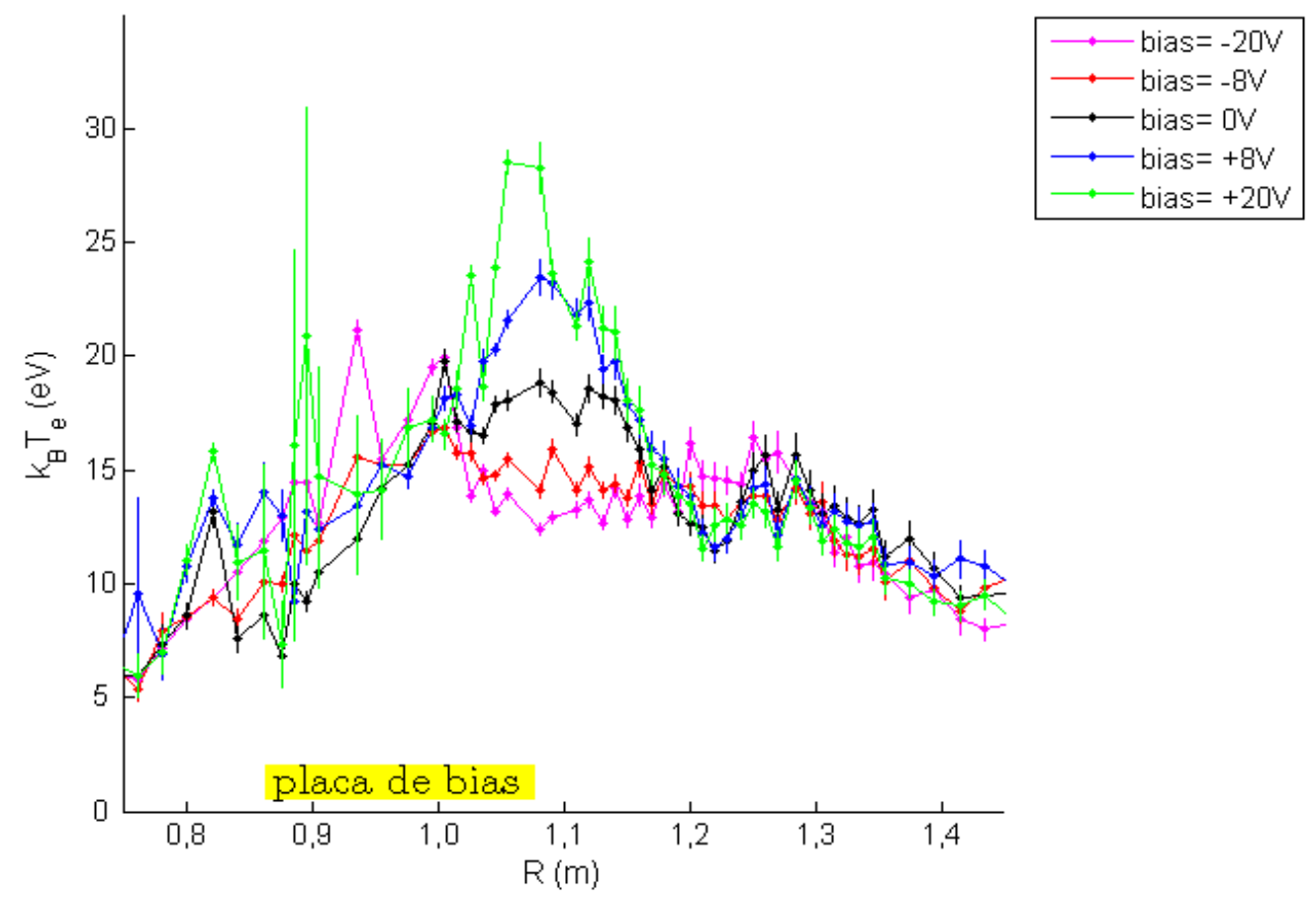

Figura 5.5: Perfis radiais da temperatura eletrônica média para cinco valores distintos de bias (-20 V, $8 \mathrm{~V}, 0,+8 \mathrm{~V} \mathrm{e}+20 \mathrm{~V})$. Todas as medidas foram feitas com sondas posicionadas na mesma posição vertical $\mathrm{z}=1,747 \mathrm{~m}$.

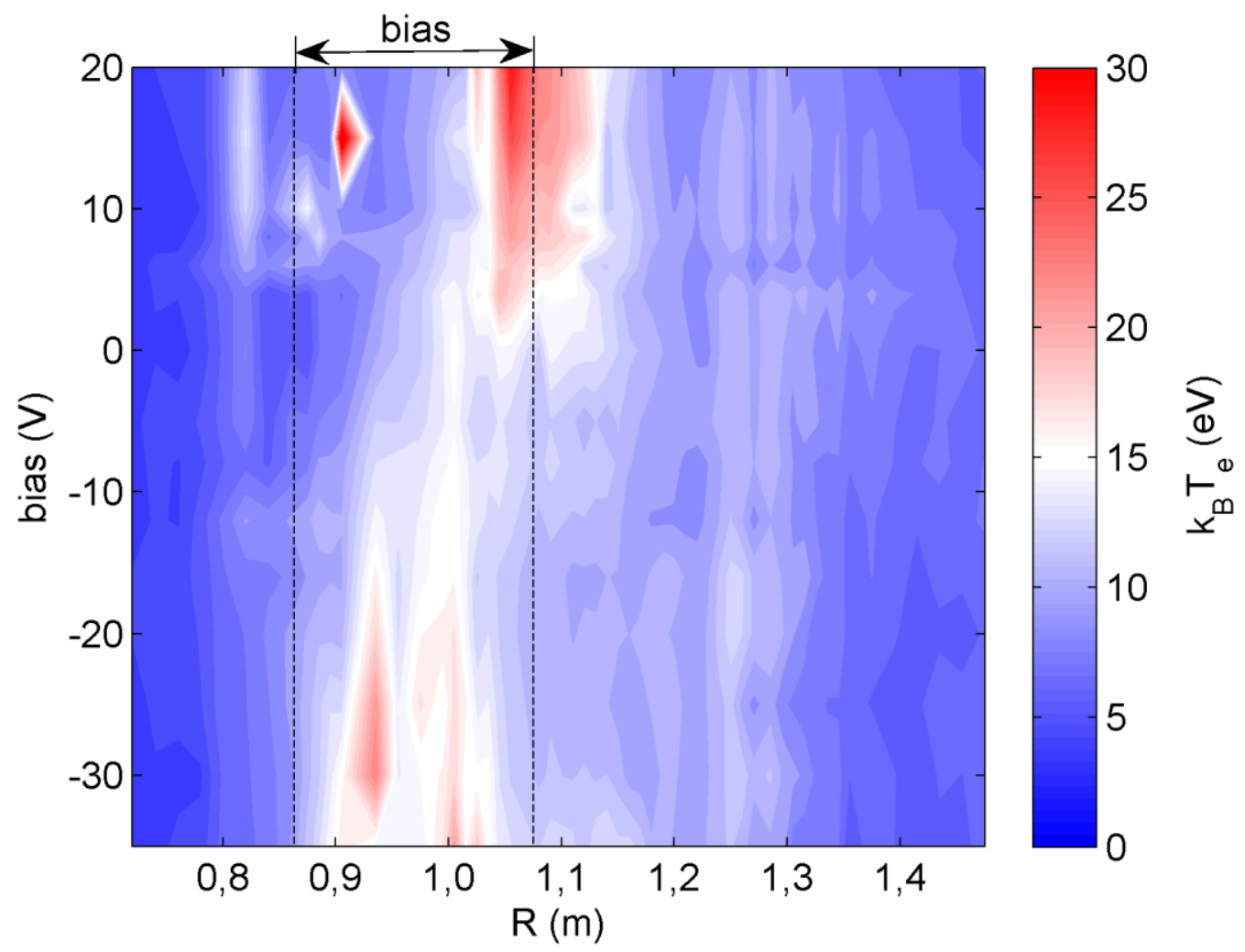

Figura 5.6: Temperatura eletrônica média como função da posição radial da sonda e do valor de bias aplicado. As linhas pontilhas marcam a região radial onde esta localizada a placa de bias. Todas as medidas foram feitas com sondas posicionadas na mesma posição vertical $\mathrm{z}=1,747 \mathrm{~m}$. 


\subsection{Turbulência}

\subsubsection{Caracterização estatística da turbulência}

A principal medida estatística da turbulência é o nível de turbulência que, para as flutuações da corrente de saturação iônica, é definido como $\sigma_{I_{s a t}} /\left|\bar{I}_{\text {sat }}\right|$ e para as flutuações do potencial flutuante é definido como $\sigma_{V_{\text {float }}} / k_{B} T_{e}$. A Figura 5.7 mostra o nível de turbulência para corrente de saturação iônica (a) e potencial flutuante (b) como função da posição radial da sonda e do valor de bias aplicado. Nessa figura observa-se que de um modo geral o nível de turbulência apresenta menor valor na região radial onde o bias é aplicado $(0,863 \mathrm{~m}<\mathrm{R}<1,075 \mathrm{~m})$. A informação mais importante relacionada aos níveis de turbulência é que estes, de um modo geral, apresentam valores menores para valores negativos de bias, mostrando que a turbulência tende a ser reduzida para valores de bias consideravelmente negativos (bias $<-15 \mathrm{~V}$ ) independentemente do valor do raio, algo já constatado por GENTLE (2010).
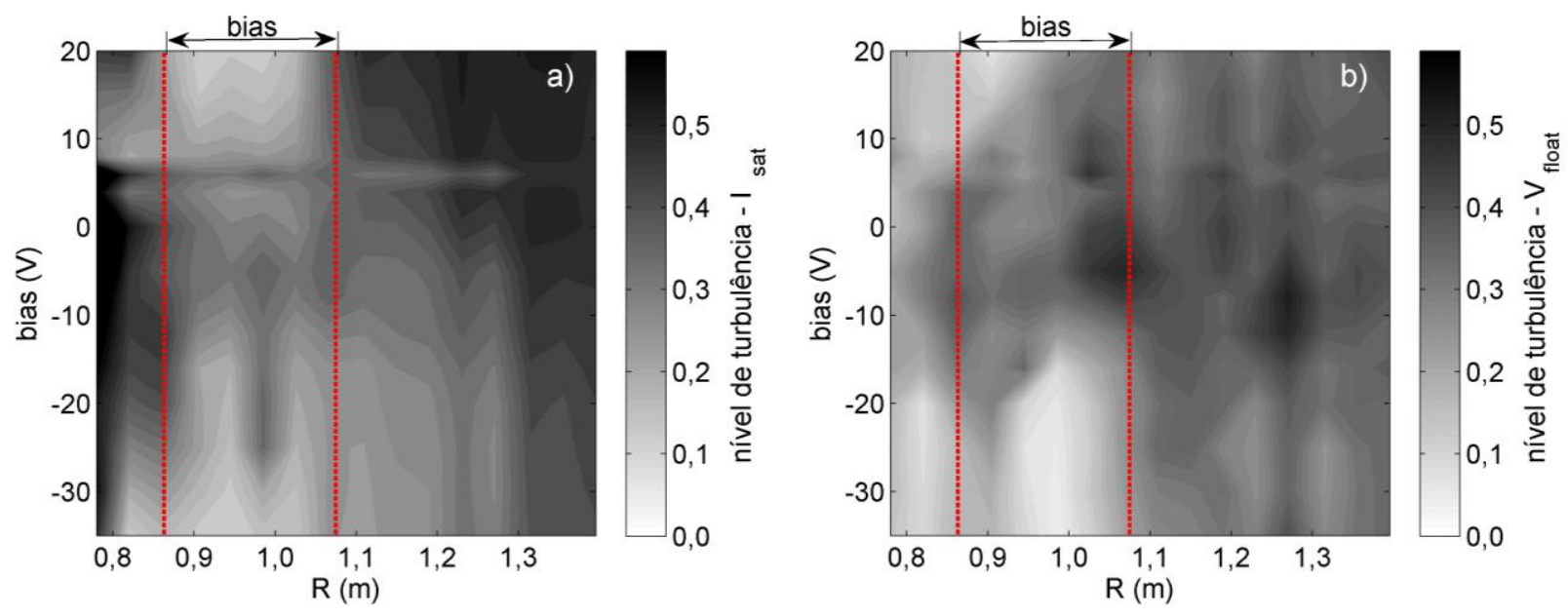

Figura 5.7: Nível de turbulência para corrente de saturação iônica (a) e potencial flutuante (b) como função da posição radial da sonda e do valor de bias aplicado. As linhas pontilhadas vermelhas marcam a região radial onde esta localizada a placa de bias. Todas as medidas foram feitas com sondas posicionadas na mesma posição vertical $\mathrm{z}=1,747 \mathrm{~m}$.

Da Figura 5.7 percebe-se também que o nível de turbulência da corrente de saturação iônica e do potencial flutuante, de modo geral, apresentam comportamentos semelhantes em relação ao valor do bias e da posição radial além de apresentarem ordens de grandeza similares. Isso pode significar que, para certos valores de bias e de 
raio o plasma do Texas Helimak satisfaz a condição de adiabaticidade dada pela equação a seguir:

$$
\frac{\sigma_{n} / \bar{n}}{\sigma_{\varphi} / k_{B} T_{e}} \cong 1
$$

sendo $\sigma_{n}$ o desvio padrão das flutuações de densidade, $\bar{n}$ a média temporal da densidade, $\sigma_{\varphi}$ o desvio padrão das flutuações do potencial do plasma, $T_{e}$ a temperatura eletrônica e $k_{B}$ a constante de Boltzmann.

Para verificar para que valores de raio e bias a condição de adiabaticidade pode ser satisfeita é preciso primeiramente considerar as flutuação de temperatura desprezíveis de modo que as relações a seguir sejam boas aproximações,

$$
\begin{aligned}
& \frac{\sigma_{\varphi}}{k_{B} T_{e}} \cong \frac{\sigma_{V_{\text {float }}}}{k_{B} T_{e}}, \\
& \frac{\sigma_{n}}{\bar{n}} \cong \frac{\sigma_{I_{\text {sat }}}}{\bar{I}} .
\end{aligned}
$$

Com isso é possível calcular o valor aproximado da relação mostrada no lado esquerdo da equação 5.1 como função do raio e dos valores de bias. O resultado, visto na Figura 5.8, mostra que a condição de adiabaticidade é razoável para qualquer valor de bias deste que $\mathrm{R}>1,09 \mathrm{~m}$, novamente indicando que a região radial $1,10 \mathrm{~m}<\mathrm{R}<$ 1,25 m é especialmente interessante para análises (uma vez que é comum supor a validade dessa relação nas teorias sobre instabilidades e turbulência de um plasma confinado magneticamente). 


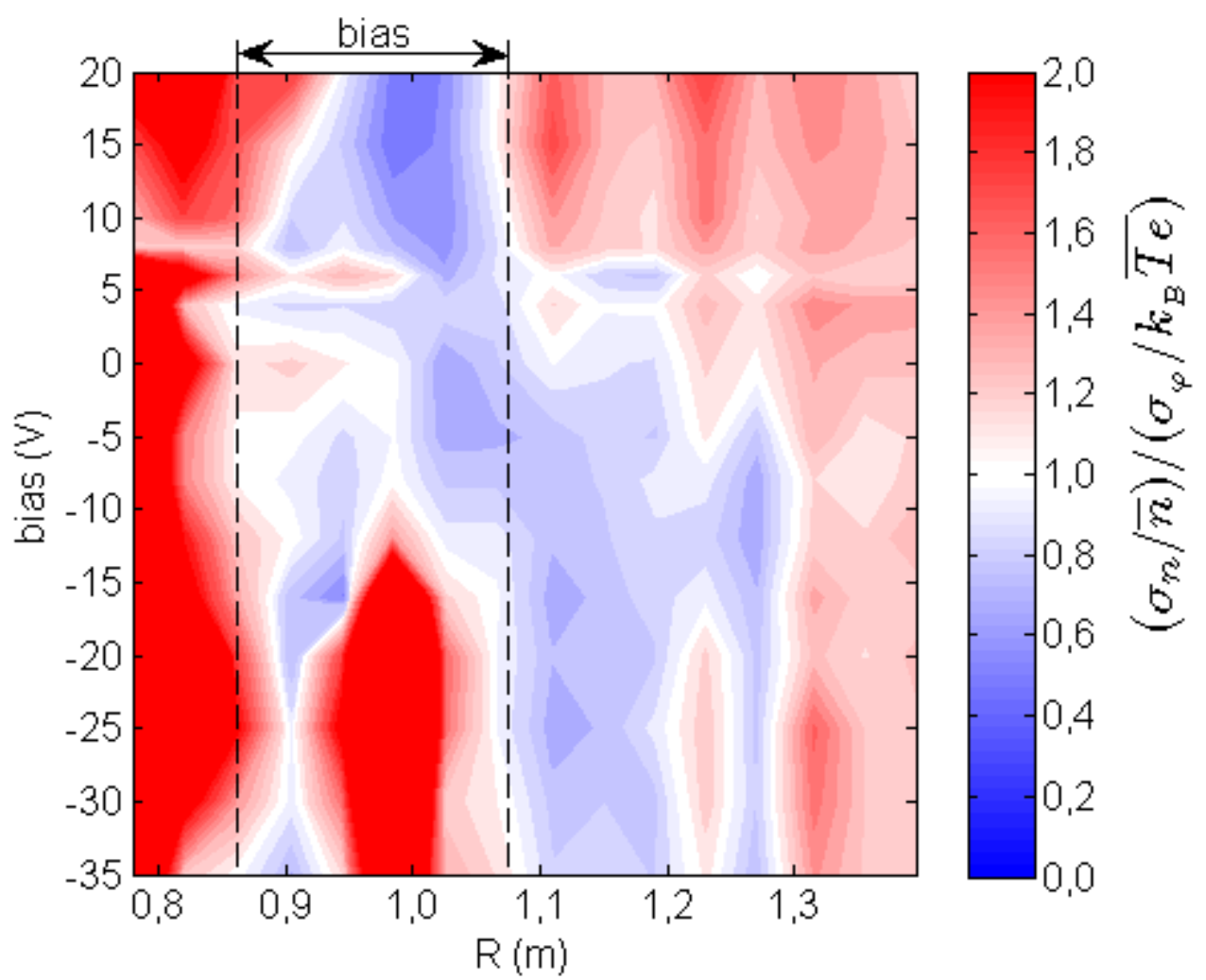

Figura 5.8: Condição de adiabaticidade, equação 5.1, como função da posição radial da sonda e do valor de bias aplicado. As linhas pontilhadas marcam a região radial onde esta localizada a placa de bias. A escala de cor foi saturada em dois de modo que as regiões mais claras indiquem onde a condição de adiabaticidade é uma boa aproximação. Todas as medidas foram feitas com sondas posicionadas na mesma posição vertical $\mathrm{z}=1,747 \mathrm{~m}$.

\subsubsection{Caracterização espectral da turbulência}

Para que se tenha uma visão geral das características espectrais da turbulência no Helimak, na região radial $1,10 \mathrm{~m}<\mathrm{R}<1,25 \mathrm{~m}$, que como já discutido neste capítulo apresenta características favoráveis para o estudo da influência do bias na turbulência, foram utilizados disparos cuja distribuição de sondas, vista na Figura 4.4, permite medidas com boa resolução radial e vertical na faixa de interesse.

Para iniciar este estudo da dependência das características espectrais com o bias externo, o espectro de potência como função do raio foi calculado para todos os valores de bias. O resultado é mostrado na Figura 5.9, para bias zero e valores positivos de bias, e na Figura 5.10, para valores negativos de bias, na forma de gráficos em que a potência espectral $(P x x)$ é representada na cor do gráfico. Ainda na Figura 5.9 e na Figura 5.10 cada um dos gráficos mostra os dados de um disparo distinto, adquirido com um determinado valor de bias. Assim nestas duas figuras são apresentadas a 
potência espectral em função do raio e da frequência para 14 valores distintos de bias: de $+15 \mathrm{~V}$ a $-35 \mathrm{~V}$.

Analisando os espectros para valores positivos de bias, Figura 5.9, observa-se espectros largos com pouca influência de modos de frequência definida (como os presentes nos espectros para bias zero). Nestes espectros nota-se que a maior concentração de energia ocorre para $f \leq 5 \mathrm{kHz}$ e para posições radiais mais internas.
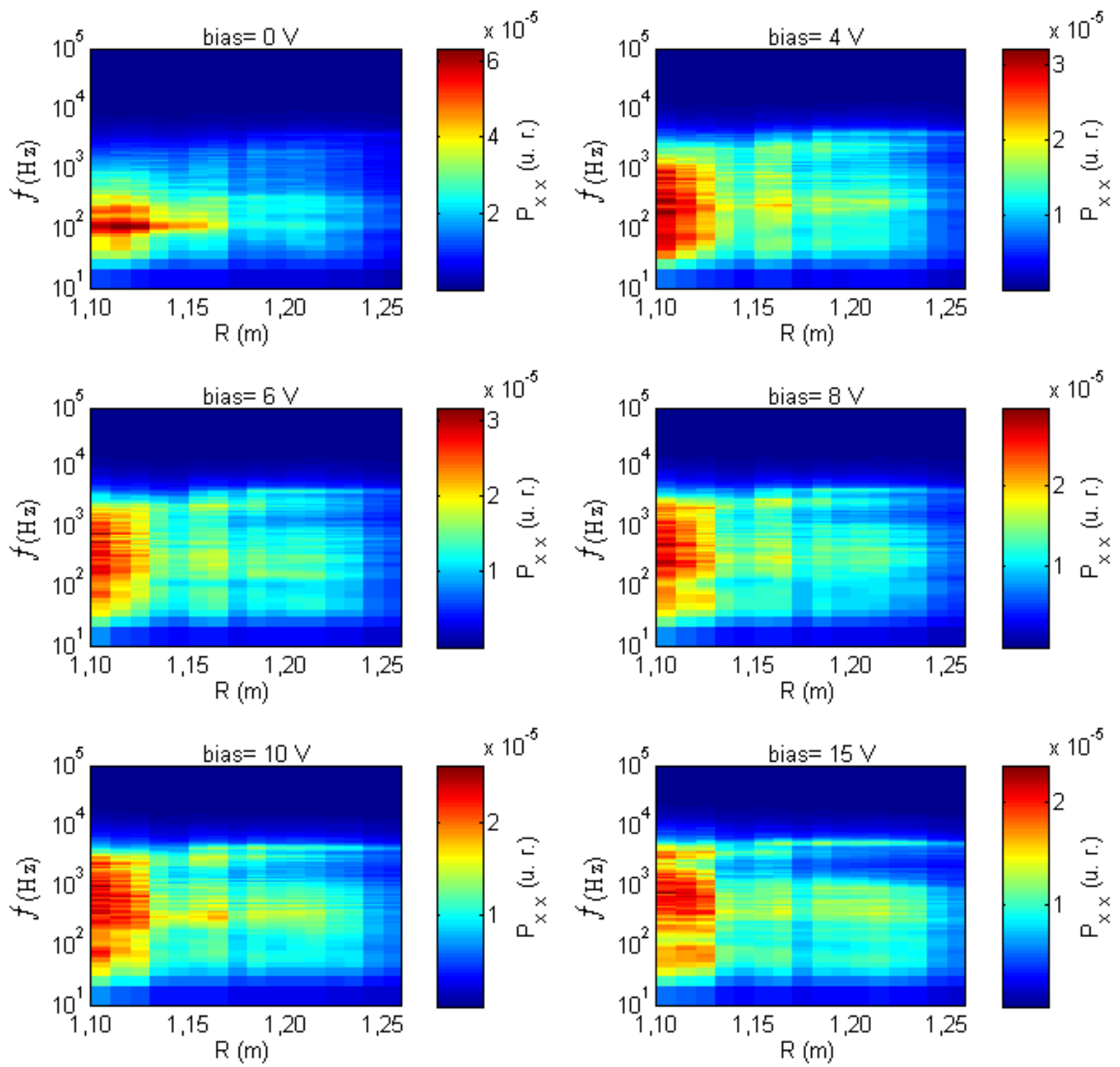

Figura 5.9: Espectros de potência, Pxx, em função do raio R para seis disparos com valores de bias zero ou positivos. Nesta figura a potência esta representada pela cor. Em todas as sondas o valor da altura é a mesma $(\mathrm{z}=1,747 \mathrm{~m})$.

Os espectros de potência em função da coordenada radial, para valores negativos de bias são apresentados na Figura 5.10. A análise destes espectros mostra inicialmente que o modo de baixa frequência, já observado para bias zero, está presente 
e com potência maior para valores intermediários de bias ( $-16 \mathrm{~V}$ e $-20 \mathrm{~V})$. Além disso, para valores de bias menores que $-20 \mathrm{~V}$ nota-se que os espectros apresentam uma concentração ainda maior de potência para frequências mais baixas, fazendo com que estes apresentam uma banda mais estreita quando comparados com os espectros para bias $\geq 0 \mathrm{~V}$. Nota-se que, a menos dos casos em que o espectro é dominado pelo modo de baixa frequência, a maior concentração de potência ocorre para posições radiais próximas a 1,18 m e não para raios mais internos como observado para valores positivos de bias. A dependência da largura espectral com o bias e a coordenada radial e a sua interpretação será melhor estudada posteriormente neste trabalho.

Observando com cuidado os espectros para bias $-25 \mathrm{~V},-30 \mathrm{~V}$ e $-35 \mathrm{~V}$, observase um modo de potência relativamente baixa e com frequência mais alta $f \cong 4 \mathrm{kHz}$ que está presente apenas para posições radiais mais internas. Na Figura 5.11 é apresentado, para bias $=-35 \mathrm{~V}$, o espectro de potência em função da frequência e do raio (a) com destaque para a faixa de frequência onde esse modo se encontra $(1 \mathrm{kHz} \leq f \leq 10 \mathrm{kHz})$. Nesta figura também são vistos os espectros de potência para dois raios específicos, $\mathrm{R}=$ $1,110 \mathrm{~m}$ (b) e R = 1,190 m (c) nos quais é possível comparar o espectro com e sem a presença deste modo. A indentificação deste modo demonstra a capacidade de resolução da análise dos espectros de potência. 

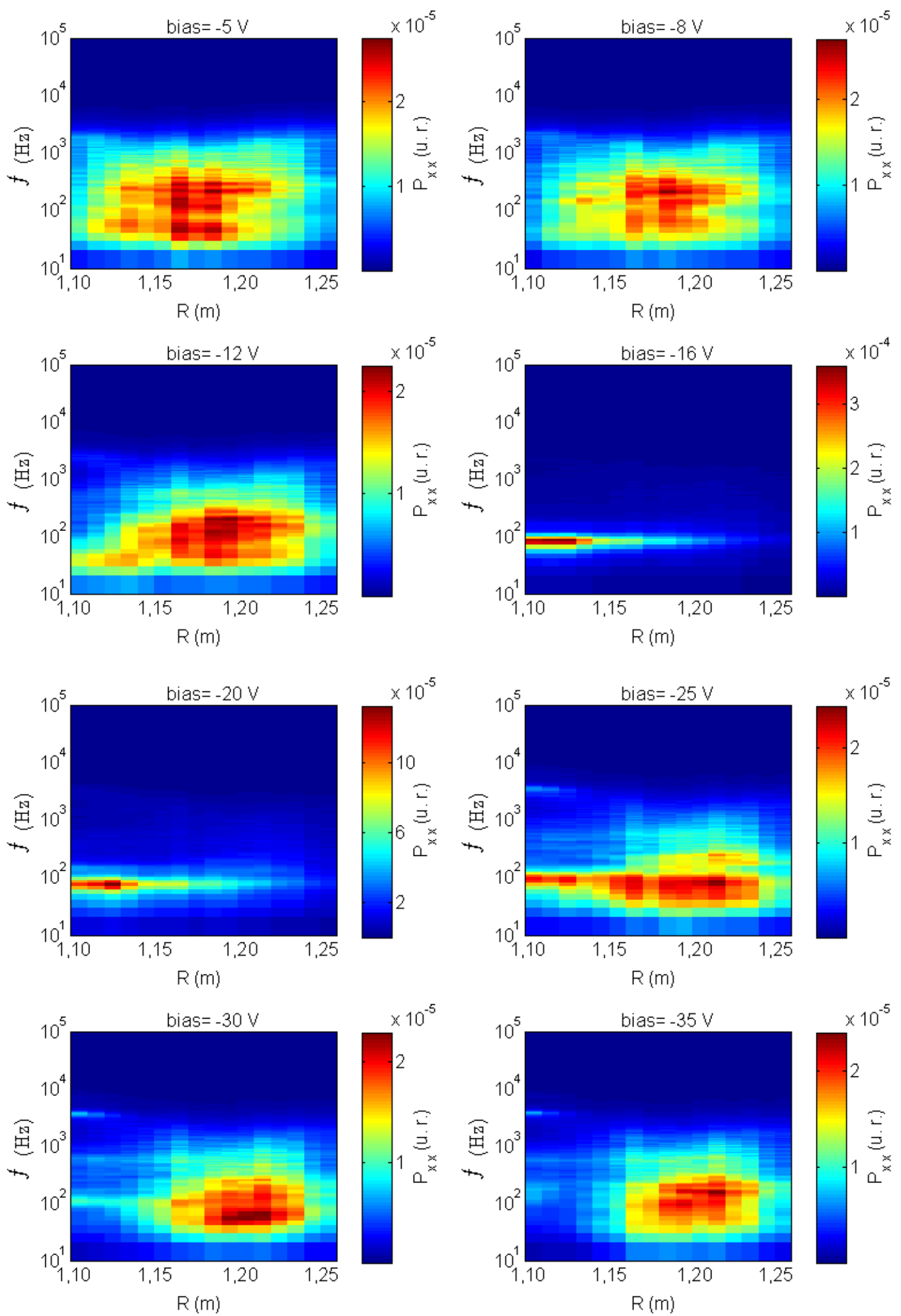

Figura 5.10: Espectros de potência, Pxx, em função do raio $\mathrm{R}$ para oito disparos com valores de bias negativos. Nesta figura a potência esta representada pela cor. Em todas as sondas o valor da altura é a mesma $(\mathrm{z}=1,747 \mathrm{~m})$. 

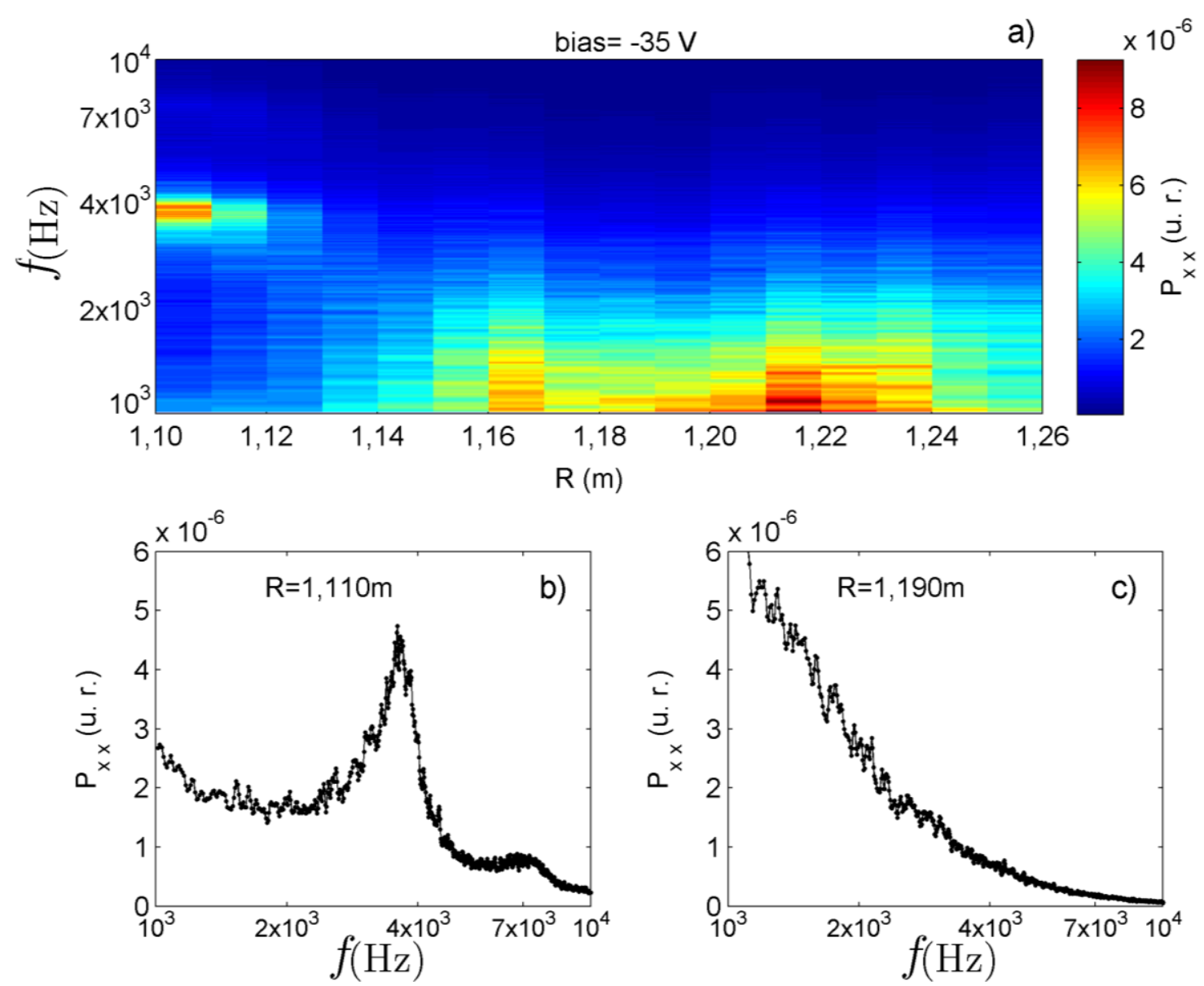

Figura 5.11: Espectro de potência, a potência esta representada pela cor, em função do raio R para disparo com bias $=-35 \mathrm{~V}$ mostrando a presença de um modo de $f \cong 4 \mathrm{kHz}$ (a). Espectros de potência para dois raios específicos, $\mathrm{R}=1,110 \mathrm{~m}$ (b) e $\mathrm{R}=1,190 \mathrm{~m}$ (c) nos quais o modo de $f \cong 4 \mathrm{kHz}$ pode ser observado com mais detalhes. Em todas as sondas o valor da altura é a mesma $(\mathrm{z}=1,747 \mathrm{~m})$.

Além da potência, o bias aplicado também pode modificar a propagação de ondas no plasma, caracterizada através do espectro de número de onda, $k(f)$. Para observar este efeito do bias nos espectros $k(f)$ a Figura 5.12 apresenta os espectros de número de onda em função do raio para quatro valores distintos de bias $(+15 \mathrm{~V}, 0,-16 \mathrm{~V}$ e $-30 \mathrm{~V})$. Nessa Figura percebe-se que os espectros de $k(f)$ para bias positivo possuem as mesmas características dos espectros para bias $=0$, Figura 4.8 (a). Outra característica interessante é que, para valores negativos de bias, $k(f)$ apresenta valores positivos para raios próximos a $\mathrm{R}=1,10 \mathrm{~m}$. Essa mudança nos valores de $k(f)$, em relação a bias $=0$, ocorre pelo fato pois o bias que é aplicado em uma placa localizada entre $\mathrm{R}=0,863 \mathrm{~m}$ e $\mathrm{R}=1,075 \mathrm{~m}$ tem um efeito maior para raios mais próximos de $\mathrm{R}=1,075 \mathrm{~m}$. 

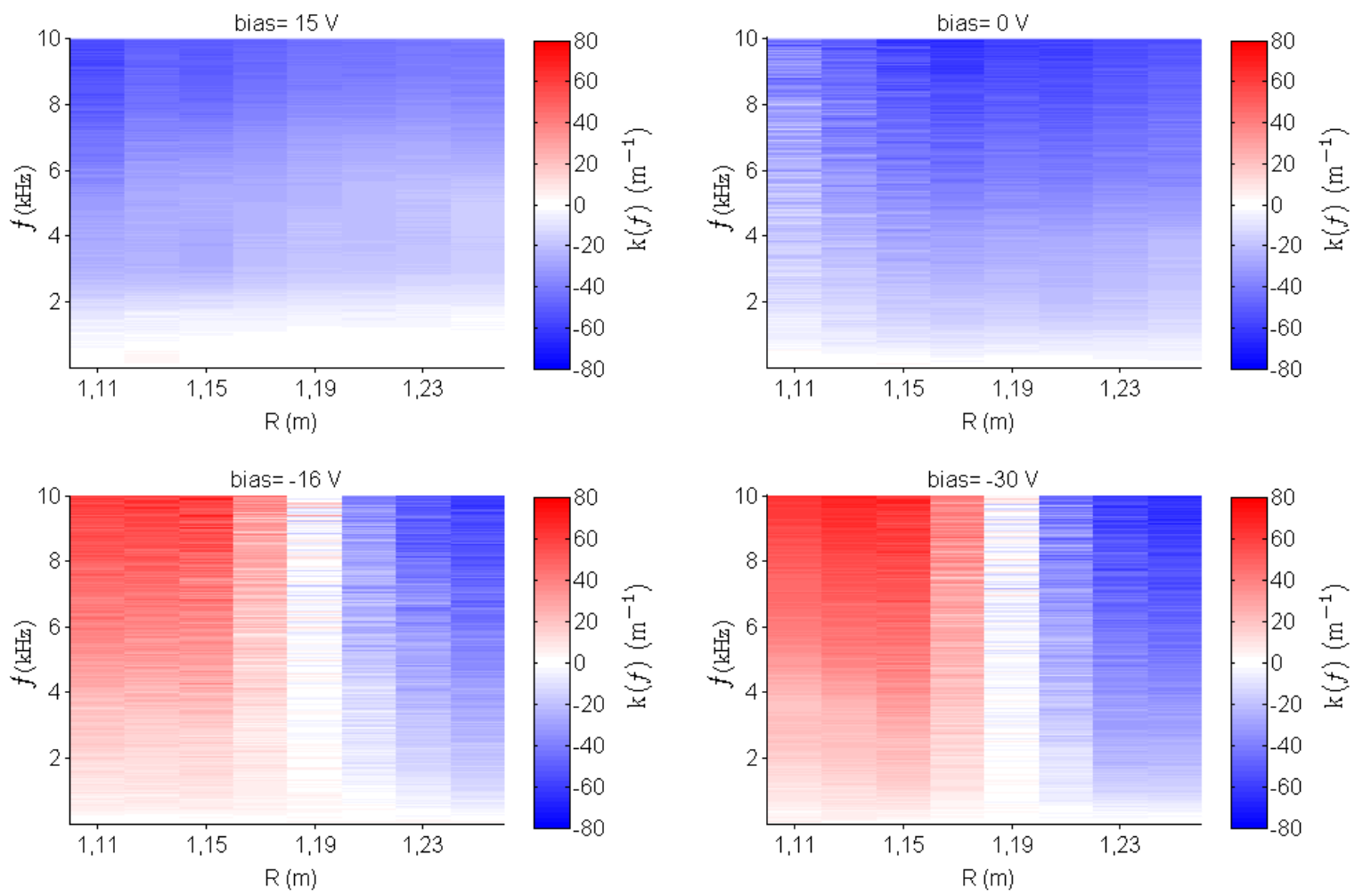

Figura 5.12: Espectros de $k(f)$ em função do raio para quatro valores distintos de bias $(+15 \mathrm{~V}, 0,-16 \mathrm{~V}$ e $30 \mathrm{~V})$. Nesta figura o valor de $k(f)$ esta representado pela escala de cor. Nota-se que para bias $<0$ ocorre a inversão da velocidade de fase.

Como a relação de $k \operatorname{com} f$ esta ligado a velocidade de fase de ondas viajando no plasma, é possível estimar o valor médio desta velocidade de fase através do espectro de $S(k, f)$, como mostrado no capítulo 3 (equação 3.10).

A Figura 5.13 mostra velocidades de fase, numa escala em cores, como função do raio e do valor do bias. Estas velocidades foram estimadas através do espectro de $S(k, f)$ para pares de sondas posicionadas em $z_{1}=1,747 \mathrm{~m} \mathrm{e} z_{2}=1,767 \mathrm{~m}(\Delta \mathrm{z}=2 \mathrm{~cm})$. Nesta figura fica claro o efeito do bias negativo, que inverte a sentido das velocidades de fase em coordenadas radiais entre $\mathrm{R}=1,11 \mathrm{~m}$ e $\mathrm{R}=1,19 \mathrm{~m}$. Nota-se que, para valores menores de bias negativos como $-5 \mathrm{~V}$ e $-8 \mathrm{~V}$, a inversão do sentido da velocidade de fase ocorre para raios menores. Para bias menor que $-16 \mathrm{~V}$ a inversão do sentido de $V_{p h}$ ocorre sempre para $\mathrm{R} \cong 1,19 \mathrm{~m}$ independente do quão negativo é o valor do bias. $\mathrm{A}$ região clara vista na Figura 5.13, na qual $V_{p h} \cong 0$, é conhecida na literatura como shear layer. 


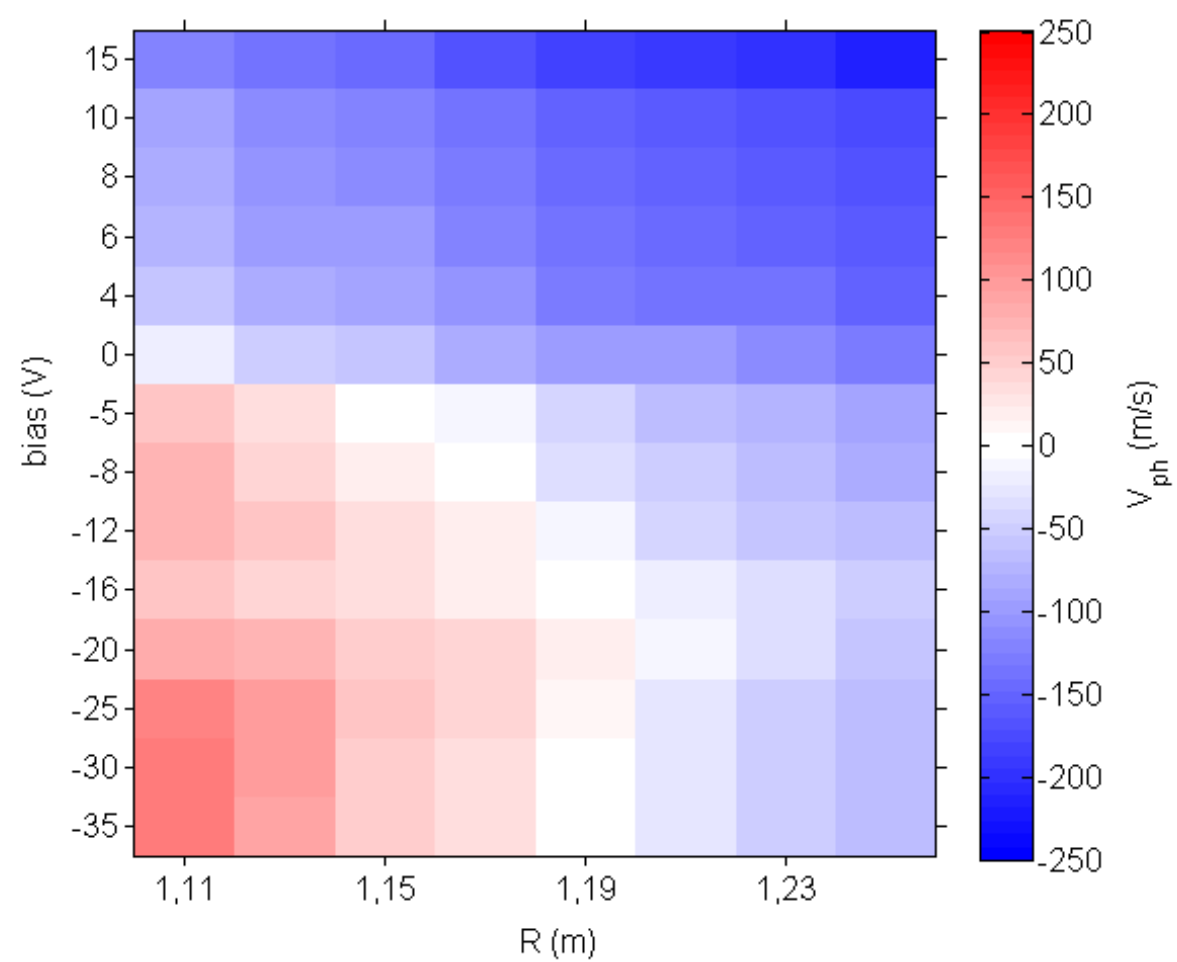

Figura 5.13: Velocidade de fase $\left(\mathrm{V}_{\mathrm{ph}}\right)$ estimada através do espectro de $S(k, f)$ em função da posição radial e do valor do bias. Nesta figura a inversão do sentido de $\mathrm{V}_{\mathrm{ph}}$ pode ser identificada.

A inversão das velocidades de fase vertical para bias negativos ocorre por um efeito direto do bias sobre o potencial do plasma. A presença do bias negativo em $0,863 \mathrm{~m} \leq \mathrm{R} \leq 1,075 \mathrm{~m}$ provoca um gradiente de potencial na direção $\mathbf{e}_{r} \mathrm{e}$, consequentemente, induz um campo elétrico na direção $-\mathbf{e}_{r}\left(-E \mathbf{e}_{r}\right)$. Este campo, por sua vez, na presença do campo magnético toroidal $\left(B_{\phi} \mathbf{e}_{\phi}\right)$ irá produzir uma velocidade de deriva elétrica $\mathbf{V}_{E}=\frac{(\mathbf{E} \times \mathbf{B})}{B^{2}}$ na direção $-\mathbf{e}_{z}$. Em contra partida, a aplicação do bias positivo deve produzir um campo elétrico na direção $\mathbf{e}_{r}$ e por consequência uma velocidade de deriva $\mathbf{V}_{E}$ na direção $\mathbf{e}_{z}$. A Figura 5.14 ilustra esse efeito do bias negativo na direção da velocidade de deriva $\mathbf{V}_{E}$ (representada por setas verdes). Nesta figura, um corte do tanque do Texas Helimak, a faixa amarela representa a região radial em que o bias é aplicado, as setas vermelhas representam o campo elétrico resultante do gradiente de potencial produzido pelo bias negativo e as setas azuis representam o campo magnético. 


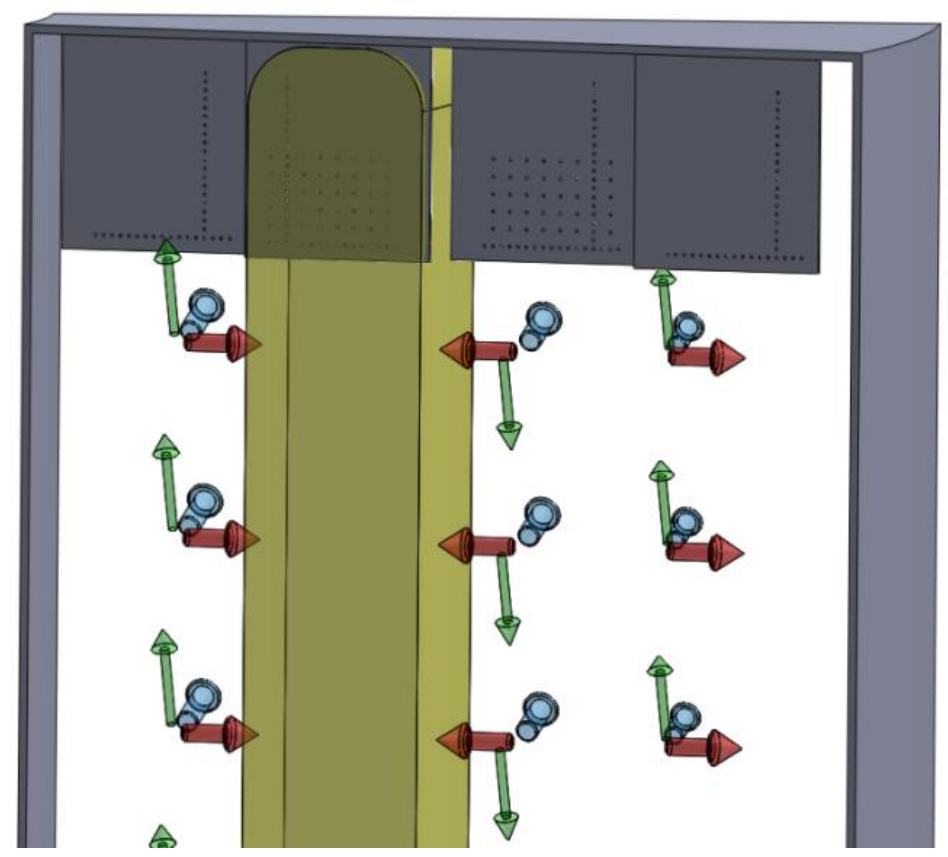

Figura 5.14: Ilustração do efeito do bias na velocidade de deriva elétrica $\mathbf{V}_{E}$ (setas verdes) na região radial próxima a placa de bias (que está posicionada na região $0,863 \mathrm{~m} \leq \mathrm{R} \leq 1,075 \mathrm{~m}$ ).

A Figura 5.15 mostra a velocidade de fluxo do plasma, medida através de espectroscopia, como função do bias e do raio. Nota-se que nesta figura também se observa a inversão da direção da velocidade para valores negativos de bias. Observa-se também que a velocidade de fluxo tem direção oposta a velocidade de fase medida no topo do tanque do Helimak, Figura 5.13. Um comentário importante que deve ser feito é que enquanto a velocidade de fase é medida em uma posição vertical bem definida (nesse caso entre $\mathrm{z}_{1}=1,747 \mathrm{~m} \mathrm{e} \mathrm{z}_{2}=1,767 \mathrm{~m}$ ) a velocidade de fluxo é uma medida da média sobre todos os dois metros de altura do tanque do Helimak. 


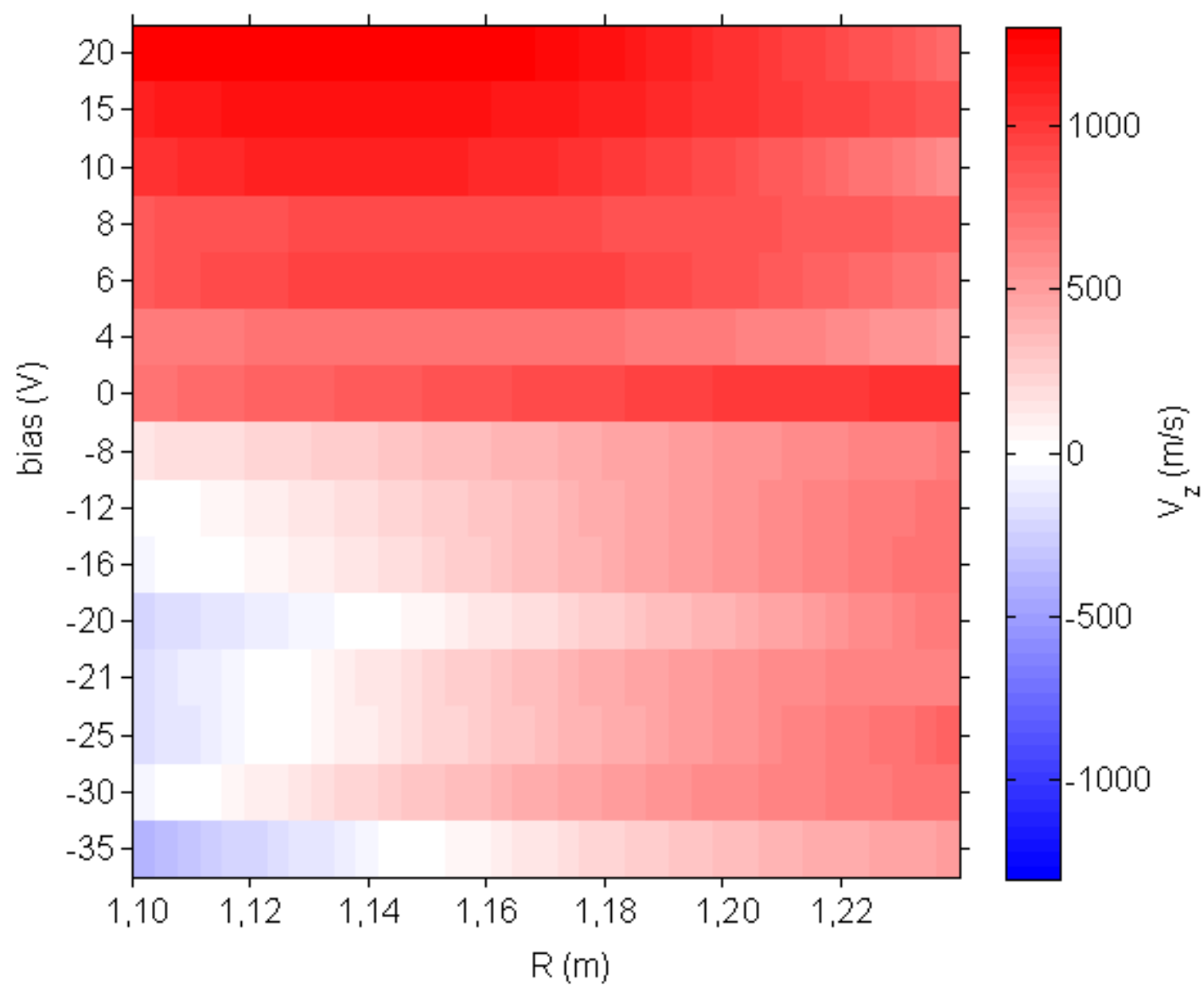

Figura 5.15: Velocidade de fluxo do plasma $\left(\mathrm{V}_{\mathrm{z}}\right)$ medida utilizando técnicas espectroscópicas. Nota-se a mudança no sentido da velocidade para valores negativos de bias".

\subsection{Considerações finais acerca do controle através do bias externo}

Considerando o que foi mostrado neste capítulo, podemos concluir em primeiro lugar que o plasma no Texas Helimak apresenta um comportamento muito diversificado, de acordo com a perturbação. Como já observado no capítulo anterior e reforçado aqui, de um modo geral todas as características do plasma dependem fortemente da coordenada radial.

Em relação ao bias, grande parte das propriedades do plasma sofre alterações significativas com a alteração do bias externo, especialmente na região radial próxima à $\mathrm{R}=1,11 \mathrm{~m}$, que fica imediatamente após a placa de bias.

Quanto aos perfis médios, o perfil radial da corrente de saturação iônica é uma exceção a regra geral, sofrendo poucas alterações com a variação do bias. Já o perfil do potencial flutuante é muito alterado pelo sinal e valor do bias na faixa radial onde a 
placa de bias está localizada. O perfil da temperatura eletrônica média, por sua vez, depende principalmente do sinal do bias aplicado, sendo essa temperatura reduzida pela imposição de valores negativos de bias e aumentada por valores positivos, sempre na região radial próxima à placa de bias.

Quanto à turbulência, a análise estatística das flutuações mostrou que a condição de adiabaticidade é uma aproximação razoável para qualquer valor de bias desde que $\mathrm{R}$ > $1,09 \mathrm{~m}$.

$\mathrm{Na}$ análise espectral foi observado que características principais do espectro de potência, como largura e presença de modos, são modificadas pelo sinal e valor do bias.

Concluindo, valores negativos e positivos de bias afetam o plasma de formas distintas. Por isso, no restante desta tese, os efeitos do bias no plasma serão analisados e interpretados separadamente para valores negativos e positivos de bias, sendo essa separação ainda discutida na seção 6.1. As alterações da velocidade de fase da turbulência e da velocidade de fluxo vertical do plasma são exemplos diretos de como tanto a intensidade do bias quanto o seu sinal afetam as características da turbulência: valores positivos de bias aumentam as velocidades já observadas para bias nulo enquanto valores negativos de bias invertem o sentido da velocidade na região radial próxima da placa.

Nos capítulos seguintes serão apresentadas análises e interpretações apenas na faixa radial $1,10 \mathrm{~m}<\mathrm{R}<1,25 \mathrm{~m}$, devido ao gradiente de densidade quase uniforme e ao maior efeito do bias sobre as características do plasma, de sua turbulência, do potencial flutuante e da velocidade de fase. 


\section{ALTERAÇÕES da TURbULÊNCIA PROVOCADAS PELO BIAS EXTERNO}

Neste capítulo serão analisadas as variações da turbulência quando da alteração do perfil radial do campo elétrico, conseguida através da imposição de um potencial elétrico externo (bias).

Nos disparos considerados neste capítulo foi utilizada a configuração de sondas representada na Figura 4.4. Essa configuração contém um conjunto de sondas distribuídas tanto na direção radial quanto na vertical concentradas no intervalo radial $1,10 \mathrm{~m}<\mathrm{R}<1,25 \mathrm{~m}$. Neste capítulo a turbulência será analisada nessa região radial que foi escolhida principalmente devido ao seu gradiente de densidade praticamente uniforme e independente do bias e por ser a região radial onde a turbulência é mais afetada pelo valor do bias.

Utilizando análise espectral e de recorrência foram identificados dois tipos de turbulência induzidos pela perturbação no campo elétrico.

Para descargas com bias positivo aplicado, a turbulência apresenta uma potência espectral mais alta mostrando que o bias positivo aumenta o nível de turbulência. Nestes casos a turbulência se caracteriza por um espectro de potência de banda larga, cuja largura de banda aumenta com o valor do bias e uma PDF não Gaussiana marcada pela presença de eventos extremos.

Por outro lado, descargas com bias negativo apresentam uma redução do nível de turbulência e da largura de banda do espectro de potência. Utilizando análise biespectral, as descargas com bias negativo foram estudadas do ponto de vista do acoplamento de ondas através do cálculo da auto-bicoerência. Para a interpretação da dependência da largura de banda do espectro de potência com a posição radial e o valor do bias foi utilizado um modelo de quatro ondas acopladas (LASHMORE-DAVIES, 2005). Este modelo descreve a evolução da amplitude de uma onda principal, do fluxo de plasma (zonal flow) e de ondas de banda lateral. Usando parâmetros experimentais, como gradiente de densidade e temperatura média, nesse modelo, o tempo de interação entre ondas foi estimado e relacionado com a largura de banda do espectro de potência. 


\subsection{Influência do bias nas características espectrais gerais da turbulência}

Como já explorado nas seções 4.2 e 5.2, a turbulência apresentada pelo plasma do Texas Helimak varia de forma importante com a posição radial e com o potencial elétrico externo (bias) aplicado. Para buscar interpretações para tal comportamento, o passo inicial é identificar algumas características gerais desta turbulência, especialmente sua dependência com o bias. A principal característica que será analisada é a largura espectral, $\Delta f$, do espectro de potência da flutuação da corrente de saturação iônica.

Para calcular a largura espectral, inicialmente o espectro de potências é reordenado de modo que as potências espectrais de cada faixa de frequência tenham ordem decrescente. Em seguida, a largura espectral percentual é determinada como a fração das potências espectrais ordenadas que devem ser somadas para que a potência integrada seja igual à metade da potencia integrada total. $\mathrm{O}$ valor da largura espectral em unidades de frequência é obtido multiplicando-se essa fração pela frequência máxima do espectro. Assim, um espectro de ruído branco tem largura espectral igual a metade da frequência máxima do espectro (que é igual à maior largura espectral possível), ao passo que o espectro de um único modo sem ruído tem largura espectral próxima de zero.

A largura espectral como função da posição radial e da altura, z, para o bias nulo (bias ground) é vista na Figura 6.1 (a). Nesta figura nota-se que $\Delta f$ aumenta com a coordenada radial $\mathrm{R}$ e, praticamente, independe da coordenada vertical z. Sendo assim, os resultados analisados a seguir apresentarão, para cada $\mathrm{R}$, a média dos valores obtidos para diferentes z, como o perfil radial de $\Delta f$, apresentado na Figura 6.1 (b). Nessa figura os pontos representam o valor médio na direção z (para cada $\mathrm{R}$ ) e a sua incerteza foi tomada como o desvio padrão dos valores na direção z. Neste perfil nota-se claramente a variação de $\Delta f$ como função da posição radial. 

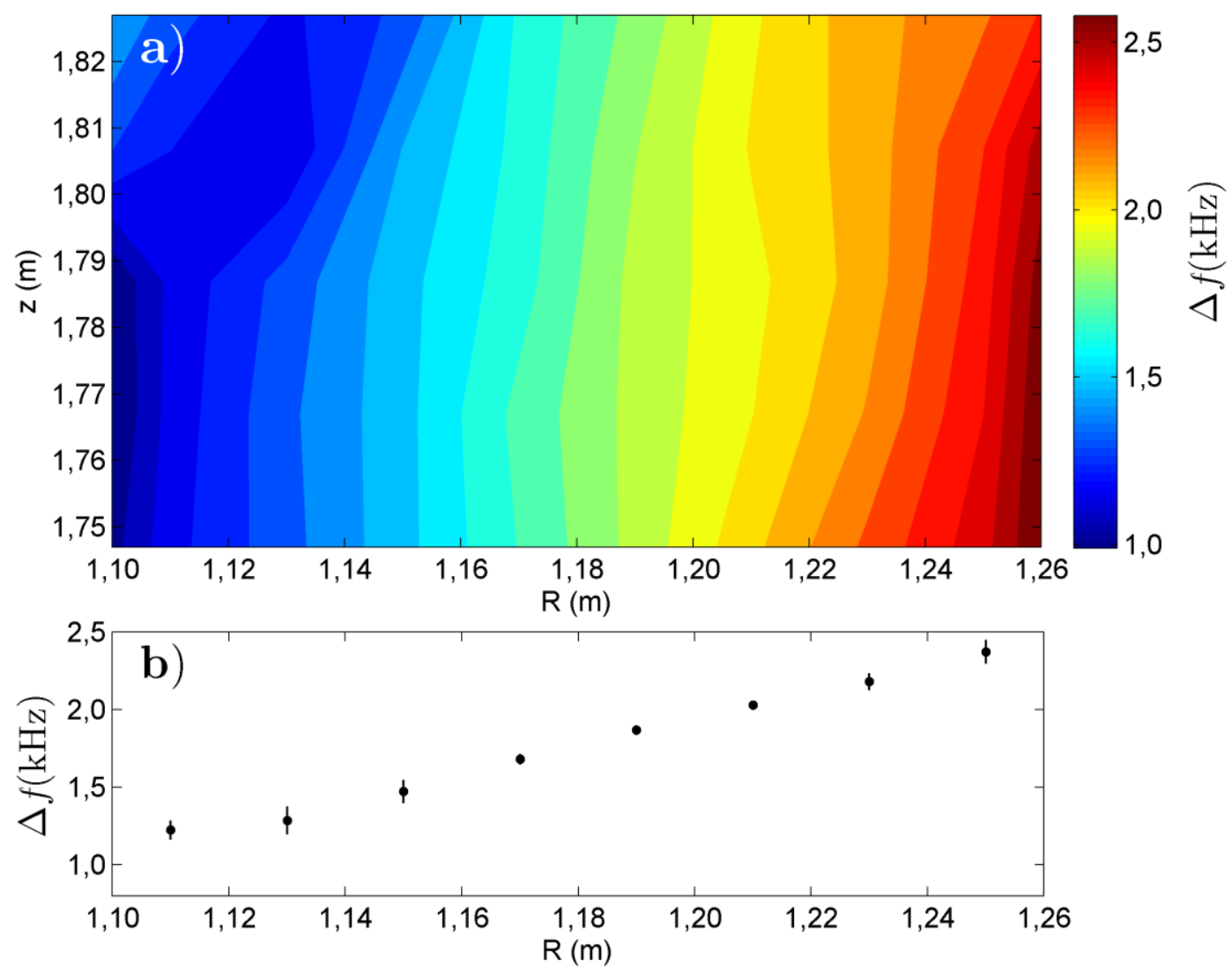

Figura 6.1: Largura espectral, $\Delta f$, dos sinais da flutuação de corrente de saturação iônica como função da posição radial e vertical (a) e largura espectral média como função da posição radial (b). O disparo mostrado foi adquirido para bias nulo.

O mesmo método utilizado para calcular o perfil radial de $\Delta f$, visto na Figura 6.1, foi empregado para descargas com valores distintos de bias. Como resultado tem-se a largura espectral em função do raio e do valor do bias, vista na Figura 6.2. Nesta figura nota-se que a largura espectral é fortemente influenciada pelo valor do bias. Primeiramente se observa que a largura espectral apresenta uma região de valores baixos (de $R \cong 1,16 m$ e bias $\cong-8 \mathrm{~V}$ até $R \cong 1,21 \mathrm{~m}$ e bias $\cong-22 \mathrm{~V}$ ). Além disso, dos resultados apresentados na Figura 6.2, fica claro que a largura espectral é maior para valores positivos de bias e que, para esses valores, a largura espectral aumenta com o valor do bias. Nota-se também que para bias $\cong-16 \mathrm{~V}$ a largura espectral cai abruptamente, especialmente para raios menores. Essa queda é explicada pela excitação de um modo de baixa frequência, já mencionado na seção 5.2.2. 


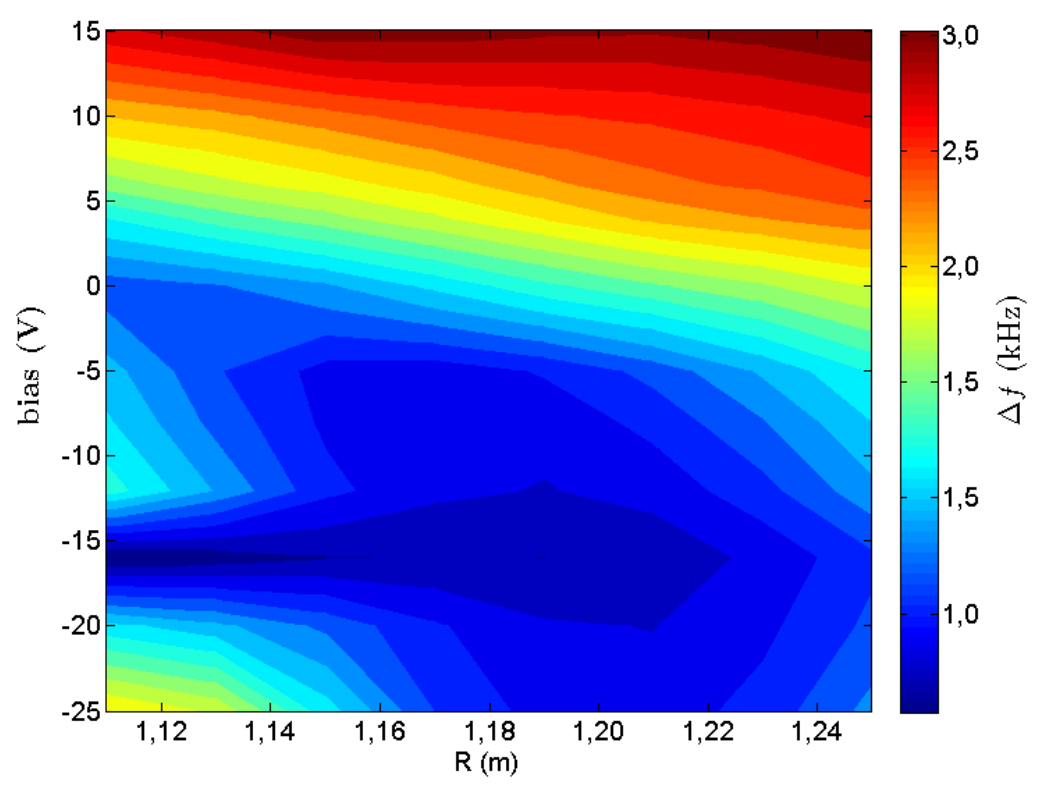

Figura 6.2: Largura espectral média, $\Delta f$, dos sinais de corrente de saturação iônica como função da posição radial e do valor de bias.

A distinção entre as características da turbulência induzidas pelo bias não afeta exclusivamente a largura espectral, mas também a própria amplitude da turbulência. $\mathrm{Na}$ Figura 6.3 a potência espectral total dos sinais de corrente de saturação iônica é mostrada como função do raio e do valor de bias. Nesta figura observa-se claramente que a potência espectral para valores positivos de bias é consideravelmente maior do que para bias nulo ou negativo.

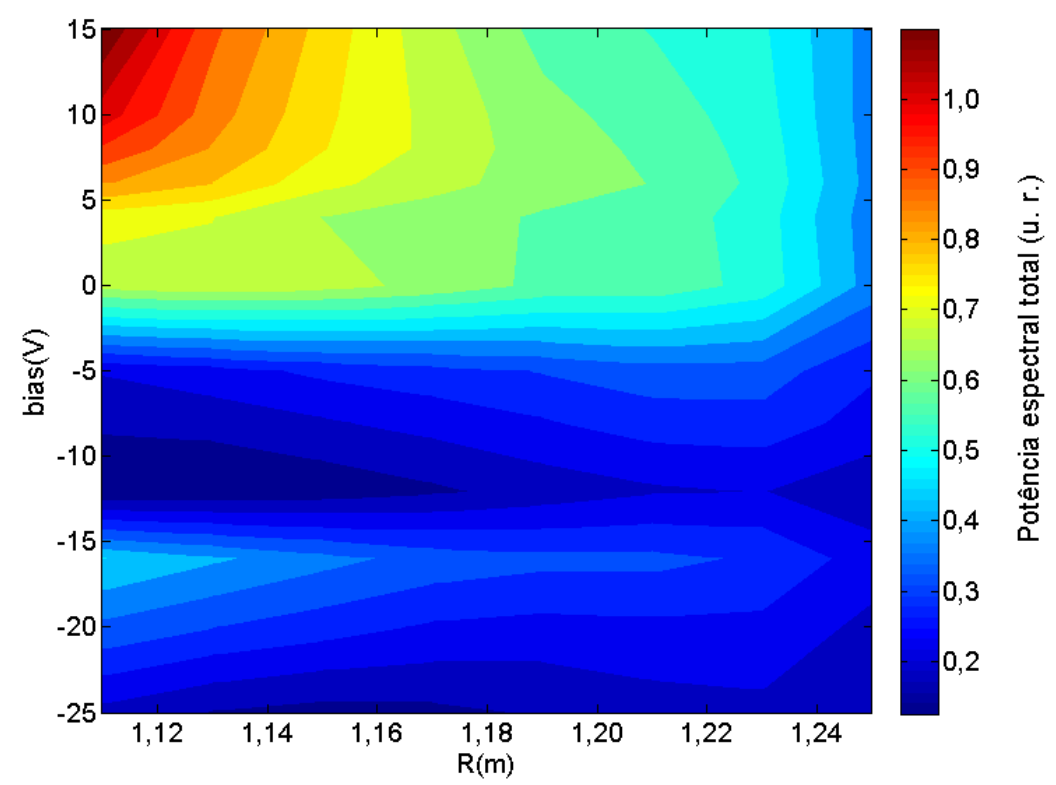

Figura 6.3: Potência espectral total dos sinais de corrente de saturação iônica como função da posição radial e do valor de bias. Essa potência representa o valor médio em relação à coordenada vertical. 
As informações apresentadas nas figuras 6.2 e 6.3 e no capítulo 5 indicam que o bias aplicado modifica as características da turbulência de forma distinta para bias negativo e positivo. Assim pode-se conjecturar que, de acordo com o sinal do bias aplicado, observam-se dois tipos de turbulência no plasma. Um tipo, induzido pelo bias negativo, apresenta amplitude e largura de banda reduzidos enquanto o outro, reforçado pelo bias positivo, apresenta potência e largura espectral maiores. Para reforçar essa conjectura diagramas de recorrência (MARWAN, 2007) foram calculados para descargas com valores distintos de bias de modo a observar em qual dos casos a turbulência apresenta uma distribuição de recorrência mais estruturada e, consequentemente, pode ser melhor representada por um modelo determinístico.

A Figura 6.4 mostra os diagramas de recorrência calculados para descargas com (a) bias $=-25 \mathrm{~V}$, (b) bias $=0 \mathrm{~V}$, (c) bias $=+15 \mathrm{~V}$ e mesma posição radial e vertical $(\mathrm{R}=$ $1,170 \mathrm{~m} \mathrm{e} \mathrm{z}=1,747 \mathrm{~m}$ ). Nota-se que o diagrama (a), correspondente a descarga com bias negativo, apresenta estruturas mais organizadas do que o diagrama (c) com bias positivo. Essa diferença indica que a turbulência de espectro estreito deve ser melhor representada por um modelo determinístico do que a turbulência de espectro largo. $\mathrm{O}$ diagrama com bias nulo (b) apresenta o comportamento intermediário. Essa observação é confirmada pela grandeza quantitativa “determinismo", DET (MARWAN, 2007; GUIMARÃES-FILHO, 2010), que está relacionado com a presença de diagonais no diagrama de recorrência. O determinismo calculado para cada um dos três casos foi: DET $=0,93(1)$ para bias $=-25 \mathrm{~V}$, DET $=0,90(1)$ para bias $=0 \mathrm{~V}$ e DET $=0,76(1)$ para bias $=+15 \mathrm{~V}$.
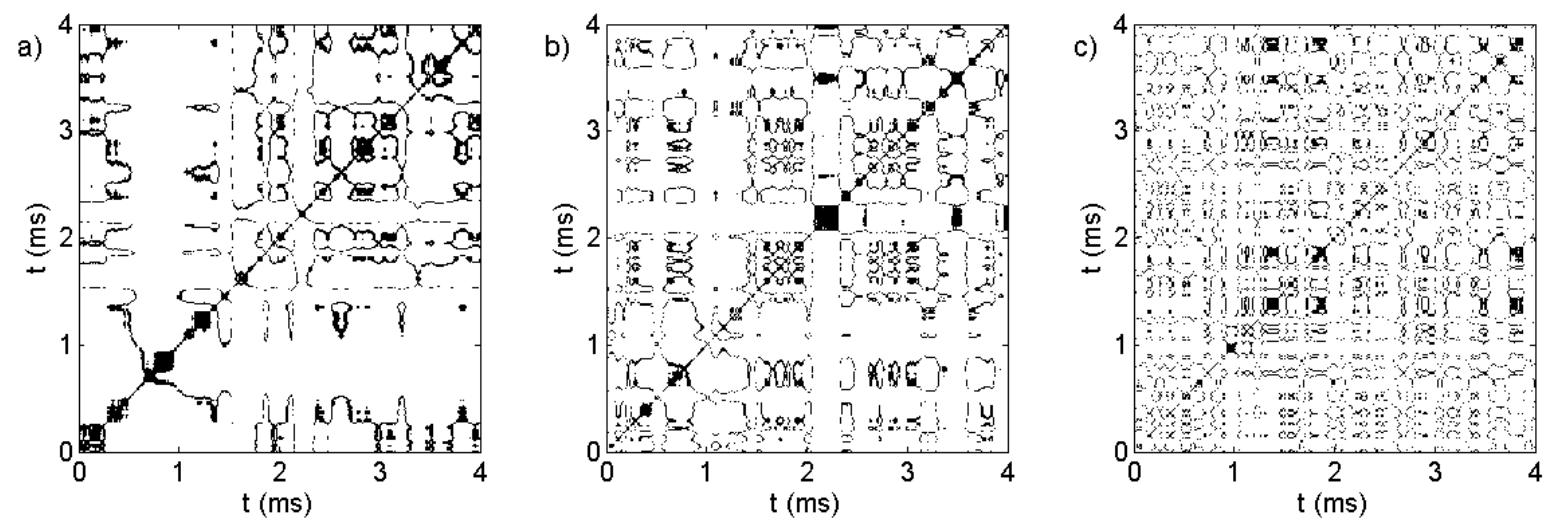

Figura 6.4: Diagramas de recorrência para descargas com (a) bias $=-25 \mathrm{~V}$, (b) bias $=0 \mathrm{~V}$, (c) bias $=+15 \mathrm{~V}$ e mesma posição radial e vertical $(\mathrm{R}=1,170 \mathrm{~m} \mathrm{e} \mathrm{z}=1,747 \mathrm{~m})$. 
Com isso, neste capítulo e no capitulo 7 , os dados serão analisados separadamente, de acordo com o sinal do bias aplicado. $\mathrm{O}$ dados adquiridos com bias positivo ou nulo serão analisados e discutidos do ponto de vistas da distribuição de probabilidades da corrente de saturação iônica. Já os dados com bias negativo serão analisados e interpretados utilizando um modelo de quatro ondas acopladas.

\subsection{Turbulência de espectro largo}

Como discutido previamente a turbulência apresentada pelo plasma em descargas perturbadas com bias positivo apresenta espectro de banda larga e dependente do valor do bias aplicado. A Figura 6.5 mostra os valores médios da largura espectral (média tomada em R e em z) como função do valor do bias. Nesta figura observa-se claramente que (para valores positivos e nulo do bias) a largura espectral aumenta quando o valor do bias aumenta.

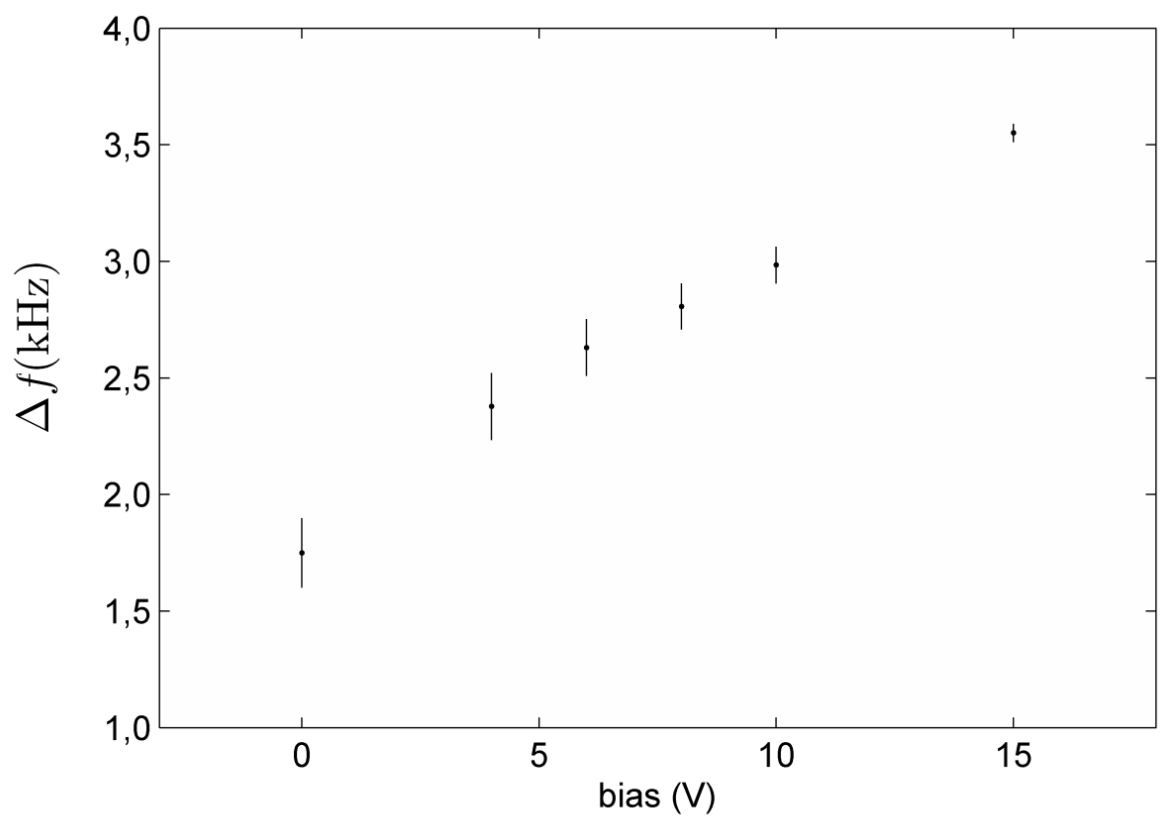

Figura 6.5: Valores médios (média em z e em R) da largura espectral, $\Delta f$, como função do valor do bias.

Além do espectro largo e dependente do valor do bias, a turbulência perturbada por valores positivos de bias também se caracteriza por apresentar uma distribuição de probabilidades não Gaussiana e muito marcada pela presença de eventos extremos. Isso pode ser verificado na Figura 6.6, na qual são apresentadas as funções de distribuição de 
probabilidade $(P D F)$ para três casos, bias $=-25 \mathrm{~V}$, nulo e $+15 \mathrm{~V}$. Nota-se que a PDF para bias $=+15 \mathrm{~V}$ (linha pontilhada) é a que apresenta os valores mais distintos da curva Gaussiana (linha vermelha), o que indica a presença mais frequente de eventos extremos.

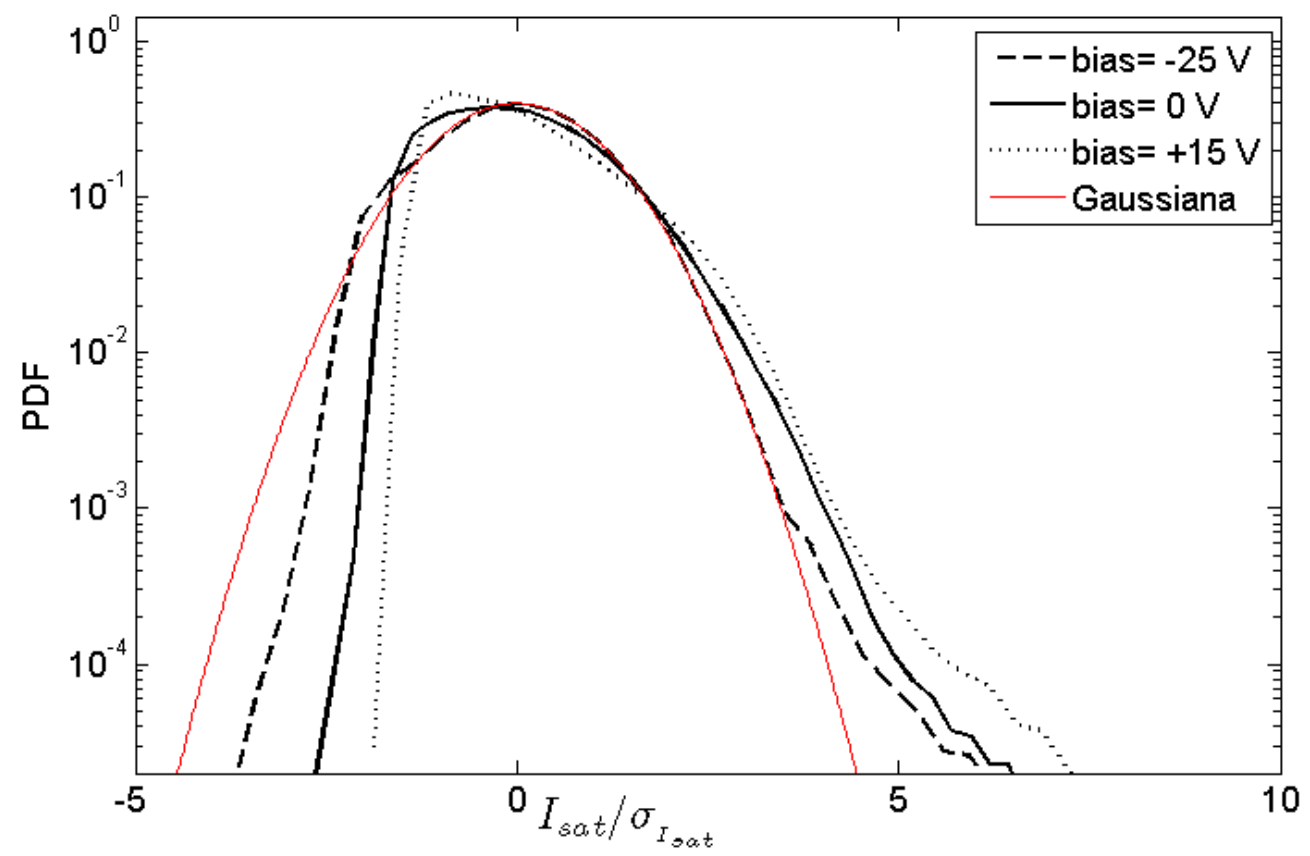

Figura 6.6: Funções de distribuição de probabilidade (PDF) dos sinais de corrente de saturação iônica para três valores de bias: $-25 \mathrm{~V}$ (linha tracejada), nulo (linha cheia) e $+15 \mathrm{~V}$ (linha pontilhada). Para fins de comparação a PDF da Gaussiana também é mostrada (em vermelho).

Uma análise direta que pode ser realizada com respeito aos eventos extremos é o estudo de sua intermitência através do intervalo de tempo entre dois picos consecutivos $(\tau)$. Para que seja possível determinar os valores de $\tau$ é preciso primeiro estabelecer um critério para definir o que será ou não considerado com um evento extremo, ou burst. Nessa análise utilizou-se critério simples de amplitudes, definindo com um evento extremo todo o pico cujo valor máximo é maior do que a média em pelo menos quatro desvios padrões. A Figura 6.7 ilustra a medida de $\tau$ e o critério de definição em um exemplo de sinal de corrente de saturação iônica medido em uma sonda posicionada em $\mathrm{R}=1,170 \mathrm{~m} \mathrm{e} \mathrm{z}=1,807 \mathrm{~m}$ e para um disparo cujo valor de bias é $+15 \mathrm{~V}$. 


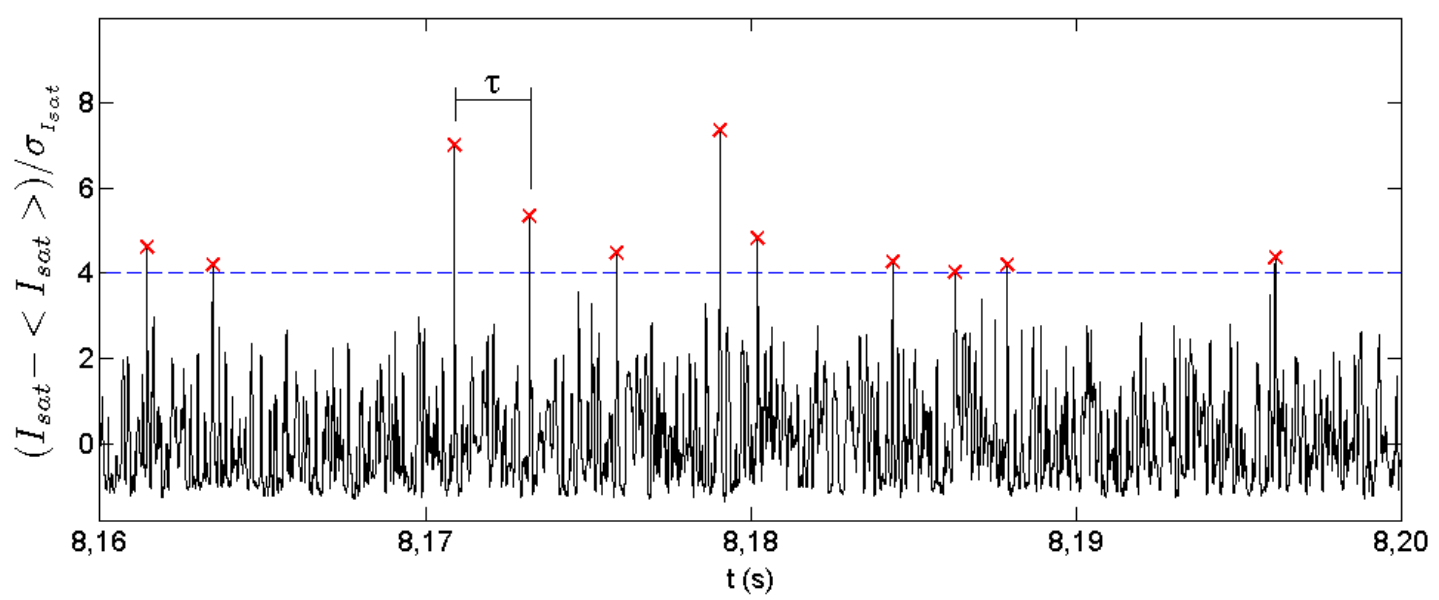

Figura 6.7: Ilustração da determinação do intervalo de tempo entre eventos extremos $(\tau)$ em um trecho de sinal de corrente de saturação iônica normalizada. Os picos que foram considerados como eventos extremos estão marcados com x e o limite de amplitudes utilizado nesta decisão está representado por uma linha tracejada azul. Neste caso a medida foi realizada por uma sonda em $\mathrm{R}=1,170 \mathrm{~m} \mathrm{e} \mathrm{z}=1,807 \mathrm{~m}$ e em um disparo com bias $=+15 \mathrm{~V}$.

Utilizando o critério discutido acima, a função de distribuição de probabilidade do intervalo de tempo entre bursts sucessivos, $P(\tau)$, foi calculada, sendo o resultado visto na Figura 6.8. Nesta figura observa-se que $P(\tau)$ pode ser descrito através de uma curva exponencial, vista em vermelho, valida para todos os valores de $\tau$.

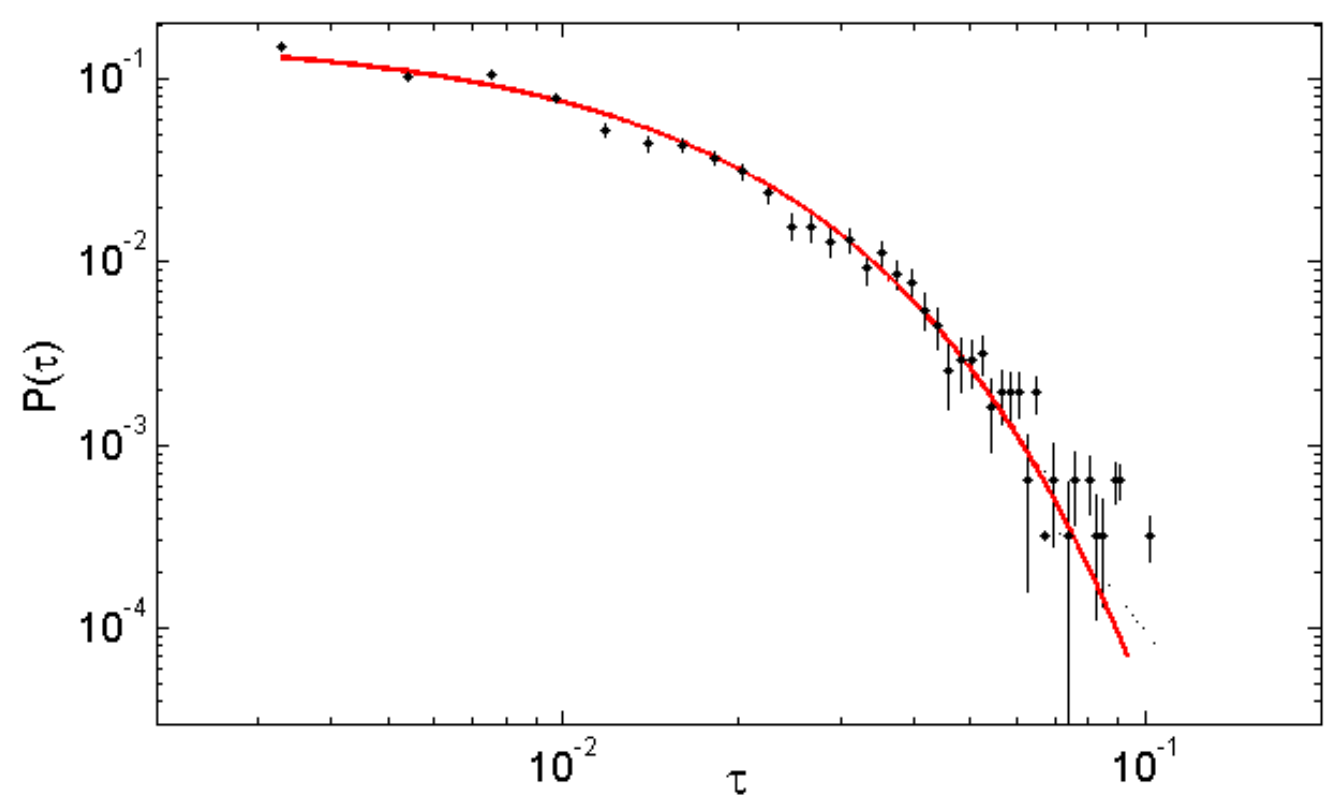

Figura 6.8: Função de distribuição de probabilidade (PDF) dos intervalos de tempo entre bursts sucessivos, $P(\tau)$ para dados de corrente de saturação iônica com bias $=+15 \mathrm{~V}$ e sondas posicionadas em $\mathrm{R}=1,170 \mathrm{~m}$. A curva em vermelho é um ajuste exponencial. 
Para as oscilações intermitentes previstas pela equação não linear de Schrödinger (BARASHENKOV, 1999), LOPES (2012) apresentou uma interpretação dinâmica para o mecanismo de injeção que produziria eventos extremos. Em (LOPES, 2012), o autor liga os picos de curta duração, na série temporal analisada, à distância, no espaço de fase do sistema, da trajetória para as variedades estáveis e instáveis. Nesse modelo espera-se que $P(\tau)$ se comporte como uma lei de potência para valores pequenos de $\tau$ e como uma exponencial para valores maiores de $\tau$. No entanto, essa previsão não foi verificada nos disparos com bias positivos do Texas Helimak, nos quais, como mostrado na Figura 6.8, $P(\tau)$ é descrita totalmente por uma exponencial. A análise dos eventos extremos observados no Texas Helimak e suas possíveis interpretações serão aprofundadas em trabalhos futuros.

\subsection{Acoplamento de ondas}

Uma forma de identificar a presença de acoplamentos não-lineares de três ondas nas descargas do Helimak é utilizando a análise espectral não linear com o cálculo da auto-bicoerência. Mais detalhes dos cálculos da auto-bicoerência $\left(b^{2}\left(f_{1}, f_{2}\right)\right)$ podem ser vistas na seção 3.2.1.

Na Figura 6.9 é visto o espectro de auto-bicoerência para a flutuação de corrente de saturação iônica de uma descarga na qual o bias é negativo $(-8 \mathrm{~V}), \mathrm{R}=1,210 \mathrm{~m}$ e $\mathrm{z}=1,747 \mathrm{~m}$. Nesta figura as frequências estão nos eixos e o valor da auto-bicoerência $\left(b^{2}\left(f_{1}, f_{2}\right)\right)$ está na escala de cinza. Na Figura 6.9, para uma melhor visualização, apenas foram mostradas as frequências menores que $1 \mathrm{kHz}$, mas para o resultado apresentado na Figura 6.10 a auto-bicoerência foi calculada até $f=4 \mathrm{kHz}$. O espectro da Figura 6.9 mostra alguns valores altos de $b^{2}$. Por exemplo, na região onde $f_{1} \cong$ $0,20 \mathrm{kHz}$ e $f_{2} \cong 0,13 \mathrm{kHz}$ se observa um valor mais alto de $b^{2}$ o que é um indício de acoplamento entre modos com frequências $0,13,0,20$ e $0,33 \mathrm{kHz}$. 


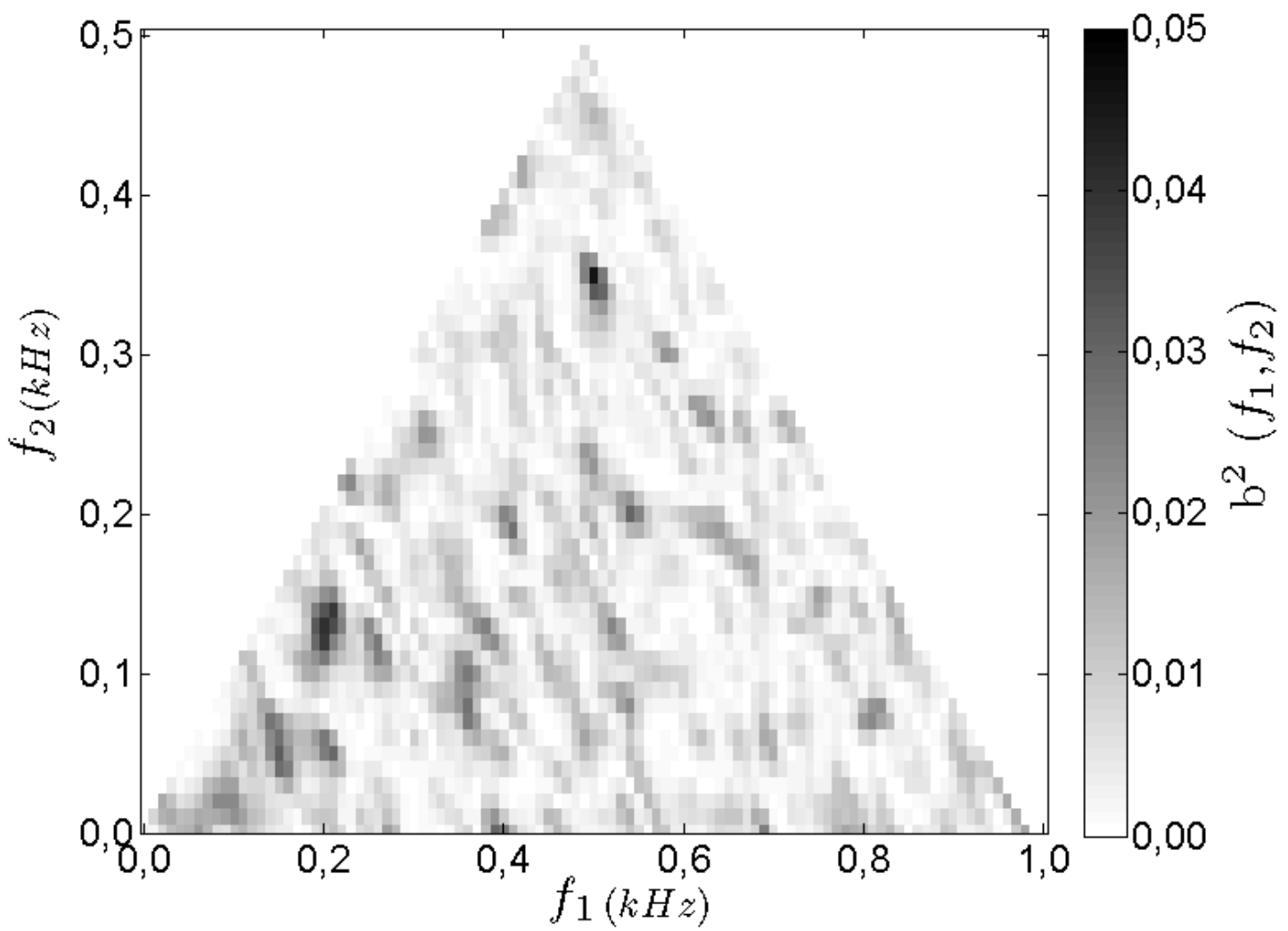

Figura 6.9: Espectro de autobicoerência para o sinal de corrente de saturação iônica medida pela sonda localizada em $\mathrm{R}=1,210 \mathrm{~m}$ e $\mathrm{z}=1,747 \mathrm{~m}$. Neste disparo o valor do bias é de $-8 \mathrm{~V}$.

Para investigar a dependência do acoplamento de ondas com a posição radial, será utilizada a auto-bicoerência soma, $S_{b}=\sum_{f_{1}} \sum_{f_{2}} b_{n 1 n 1 n 1}^{2}\left(f_{1}, f_{2}\right)$, a qual reflete o acoplamento de ondas em todas as frequências. A Figura 6.10 (a) mostra o perfil radial de $S_{b}$, tomando-se a média dos valores calculados de $S_{b}$ nas diversas sondas posicionadas em diferentes posições verticais. Nesta figura nota-se que, para $\mathrm{R}<$ 1,18 m, a auto-bicoerência soma decresce a medida que a posição radial aumenta.

Comparando a dependência de $S_{b}$ e da largura espectral $(\Delta f)$, vistas na Figura 6.10 (b), com a posição radial observa-se que ambas são similares. Isto pode ser interpretado como uma evidência de que o aumento da largura espectral pode ser uma consequência do acoplamento de modos. Assim, na seção a seguir será utilizado um modelo de acoplamento de ondas para interpretar a dependência da largura espectral com a posição radial e o valor de bias. 

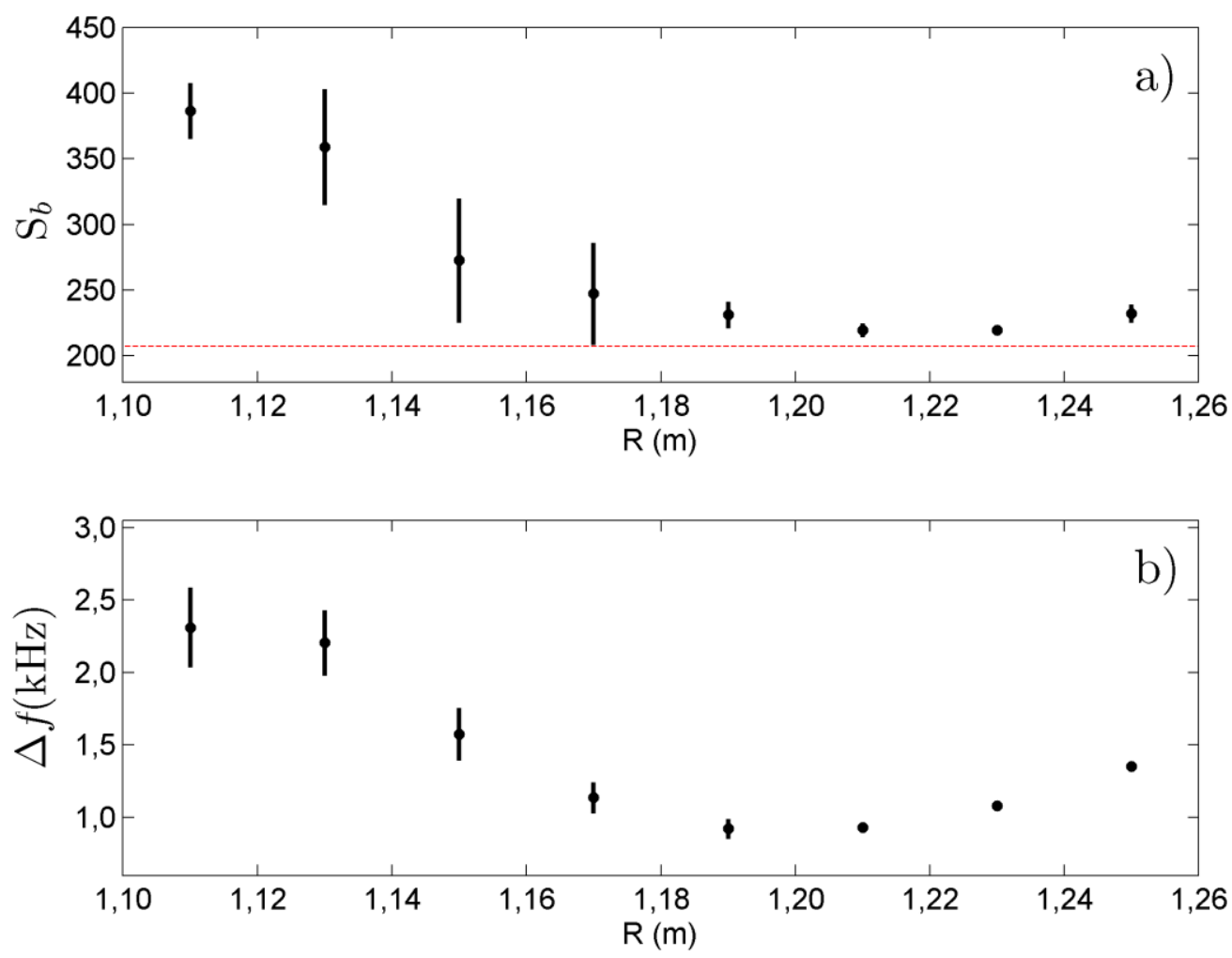

Figura 6.10: Média dos valores verticais da (a) auto-bicoerência soma, $S_{b}$, e (b) largura espectral como função da posição radial para uma descarga com bias $=-25 \mathrm{~V}$. A incerteza de cada ponto foi estimada através do desvio padrão dos valores obtidos no conjunto de seis sondas distribuídas verticalmente. A linha tracejada vermelha indica a soma da auto-bicoerência para um sinal randômico.

\subsubsection{Interpretação teórica: modelo de quatro ondas acopladas}

A turbulência pode ser descrita aproximadamente através do acoplamento de um número finito de ondas (HASEGAWA, 1979). Nesta seção, será introduzido um modelo de quatro ondas acopladas (LASHMORE-DAVIES, 2005) para descrever a turbulência do plasma no Texas Helimak com bias negativo aplicado. Este modelo considera uma onda principal que ao se acoplar com o fluxo de plasma (zonal flow) excita ondas de banda lateral. Caso o tempo de interação seja curto o suficiente, as ondas de banda lateral, podem então tomar o lugar da onda principal e se acoplar novamente com o fluxo de plasma, excitando outras ondas, levando a um efeito cascata. Assim, o modelo de quatro ondas acopladas será utilizado para relacionar a largura espectral da turbulência e o tempo de interação entre as ondas do modelo. 
Este modelo se baseia na equação de Charney Hasegawa Mima generalizada, GCHME, (CHARNEY, 1971; HASEGAWA, 1978; SMOLYAKOV, 2000) que descreve ondas de deriva eletrostática em plasmas magneticamente confinados:

$$
\left(\frac{\partial}{\partial t}+\mathbf{V}_{0} \cdot \nabla+\mathbf{V}_{d} \cdot \nabla\right) \frac{e \tilde{\phi}}{T_{e}}-\left(\frac{\partial}{\partial t}+\mathbf{V}_{0} \cdot \nabla+\tilde{\mathbf{V}}_{E} \cdot \nabla\right) \rho_{s}^{2} \nabla_{\perp}^{2} \frac{e \phi}{T_{e}}=0 .
$$

Nesta equação, $\phi$ é o potencial eletrostático total $\phi=\bar{\phi}+\widetilde{\phi}$, onde $\bar{\phi}$ é a parte média (na direção z) e $\tilde{\phi}$ representa a flutuação. A velocidade $\mathbf{V}_{0}=\hat{\mathbf{e}}_{x} \times \nabla \bar{\phi} / B$ é a parte média (na direção $\mathrm{z}$ ) da deriva $\mathbf{E} \times \mathbf{B}$ e está ligada ao zonal flow, onde o campo magnético de equilíbrio é $\mathbf{B}=B \hat{\mathbf{e}}_{x}$. A velocidade de deriva diamagnética é $\mathbf{V}_{d}=V_{d} \hat{\mathbf{e}}_{z}$, sendo $V_{d}=c_{s}^{2} /\left(\Omega_{i} L_{n}\right), L_{n}$ é o comprimento característico da densidade de equilíbrio, $L_{n}^{-1}=(1 / \bar{n})|d \bar{n} / d y|$, a velocidade $\tilde{\mathbf{V}}_{E}=\hat{\mathbf{e}}_{x} \times \nabla \tilde{\phi} / B$ esta ligada à flutuação da deriva $\mathbf{E} \times \mathbf{B}$ e $\rho_{s}=c_{s} / \Omega_{i}$ é o raio de giro acústico dos íons. A normalização do tempo utilizada é $t \rightarrow\left(\rho_{s} c_{s} / a^{2}\right) t$, onde $c_{s}=\sqrt{k_{B} T_{e} / m_{i}}$ é a velocidade acústica. Nestas equações $(x, y, z)$ representam, respectivamente, as coordenadas toroidal, radial e vertical do Helimak.

A dedução das equações que descrevem a evolução temporal das ondas a partir da Eq. 6.1 pode ser vista em (LASHMORE-DAVIES, 2001).

O modelo utilizado para interpretar os dados do Texas Helimak é simplificado considerando que, inicialmente, exista apenas uma onda de deriva principal, monocromática e de amplitude finita, cuja frequência e número de onda são dados por $\left(\omega_{0}, \mathbf{k}_{0}\right)$, onde:

$$
\begin{aligned}
\mathbf{k}_{0} & =\left(0, k_{y}, k_{z}\right), \\
\omega_{0} & =\frac{\alpha k_{z}}{1+\rho^{2} k_{0}^{2}}, \\
k_{0} & =k_{y}^{2}+k_{z}^{2},
\end{aligned}
$$

Supondo também a presença de um fluxo de plasma (zonal flow), cujas variações temporais e espaciais são caracterizadas por $(\omega, \mathbf{q})$, com número de onda $\mathbf{q}=q \hat{\mathbf{e}}_{y}$. Supondo-se ainda que a variação desse fluxo no tempo ocorre em uma escala muito 
maior que $\omega_{0}^{-1}$, a flutuação deste zonal flow pode então se acoplar com a onda principal de amplitude finita para produzir ondas de deriva de banda lateral cujos números de onda são $\mathbf{k}_{ \pm}=\mathbf{k}_{0} \pm \mathbf{q}=\left(0, k_{y} \pm q, k_{z}\right)$. Estas ondas de banda lateral, em contrapartida, podem se acoplar com a onda principal para reforçar o fluxo de plasma (zonal flow), fechando assim um laço realimentado. Este é o mecanismo por traz da instabilidade modulacional, a qual tem sido estudada por LASHMORE-DAVIES (1976 e 1997) para fluidos e plasmas magnetizados.

Introduzindo as amplitudes da onda principal, fluxo de plasma e ondas de banda lateral: $A_{0}, B, a \pm$, respectivamente, tem-se que, para a onda principal

$\tilde{\phi}_{0}=A_{0}(t) \exp \left(i k_{y} y+i k_{z} z-i \omega_{0} t\right)+c . c .$,

e para as ondas de banda lateral

$\tilde{\phi}_{ \pm}=a_{ \pm}(t) \exp \left(i\left(k_{y} \pm q\right) y+i k_{z} z-i \omega_{ \pm} t\right)+c . c .$,

sendo $\omega_{ \pm}$as frequências não perturbadas das ondas derivada dadas por

$\omega_{ \pm}=\frac{\alpha k_{z}}{1+\rho^{2} k_{ \pm}^{2}}$

onde $k_{ \pm}=\left(k_{y} \pm q\right)^{2}+k_{z}^{2}$.

A velocidade do fluxo (ligada ao zonal flow) é assumida então da seguinte forma:

$\mathbf{V}_{0}=\frac{\rho_{s}}{a} c_{s} \hat{e}_{z}[i q B(t) \exp (i q y)+c . c]$.

Substituindo as expressões das ondas (equações 6.5 e 6.8) na equação 6.1, as equações que descrevem a evolução temporal das amplitudes da onda principal, do fluxo de plasma e das ondas de banda lateral são:

$$
\frac{d A_{0}}{d t}=-\Omega_{0} \frac{\left[1+\left(k_{+}^{2}-q^{2}\right) \hat{\rho}^{2}\right]}{\left(1+k_{0}^{2} \hat{\rho}^{2}\right)} a_{+} B^{*}+\Omega_{0} \frac{\left[1+\left(k_{-}^{2}-q^{2}\right) \hat{\rho}^{2}\right]}{\left(1+k_{0}^{2} \hat{\rho}^{2}\right)} a_{-} B
$$




$$
\begin{aligned}
& \frac{d a_{+}}{d t}+i \delta_{+} a_{+}=\Omega_{0} \frac{\left[1+\left(k_{0}^{2}-q^{2}\right) \hat{\rho}^{2}\right]}{\left(1+k_{+}^{2} \hat{\rho}^{2}\right)} A_{0} B, \\
& \frac{d a_{-}}{d t}+i \delta_{-} a_{-}=-\Omega_{0} \frac{\left[1+\left(k_{0}^{2}-q^{2}\right) \hat{\rho}^{2}\right]}{\left(1+k_{-}^{2} \hat{\rho}^{2}\right)} A_{0} B^{*}, \\
& \frac{d B}{d t}=\Omega_{0} \frac{\left(k_{+}^{2}-k_{0}^{2}\right)}{q^{2}} a_{+} A_{0}^{*}-\Omega_{0} \frac{\left(k_{-}^{2}-k_{0}^{2}\right)}{q^{2}} a_{-}^{*} A_{0},
\end{aligned}
$$

onde $\delta_{ \pm}=\omega_{ \pm}-\omega_{0}$ e $\Omega_{0}=q k_{z}$.

Para interpretar as características do espectro da turbulência e sua dependência com a coordenada radial e com o valor do bias através deste modelo de acoplamento de ondas, as equações 6.9-6.12 do sistema foram integradas utilizando informações e dados experimentais para obter os parâmetros de entrada.

Esses parâmetros de entrada foram obtidos da seguinte forma: O campo magnético, $B$, em Tesla foi obtido diretamente da equação $B(R)=0,077 / R$, sendo $R$ a posição radial dada em metros. A temperatura eletrônica foi suposta constante e uniforme $\left(T_{e} \cong 13 \mathrm{eV}\right)$, vide Figura 4.1 (c). O comprimento característico da máquina é igual à largura do vaso, $a=1 \mathrm{~m}$. A massa do íon (Argônio) é: $m_{i}=6,67 \times 10^{-26} \mathrm{~kg}$. O comprimento característico da densidade de equilíbrio, $L_{n}$, foi obtido como função do valor de bias e das posições radial e vertical através dos perfis radiais médios da corrente de saturação iônica adotando-se que $L_{n}=\frac{\bar{n}}{d \bar{n} / d R} \cong \frac{\bar{I}_{s a t}}{d \bar{I}_{s a t} / d R}$, o que implica em desprezar as variações da temperatura ao longo do raio.

O comprimento de onda vertical, $k_{z}$, foi obtido, também em função do valor do bias e das posições radial e vertical, através dos espectros de $S(k, f)$, vide Figura 4.9. Como o comprimento de onda na direção radial não pode ser medido de forma precisa, ele foi adotado como $k_{y}=k_{z} / 3$. O numero de onda do zonal flow, $q$, foi obtido para cada valor de bias através de um ajuste senoidal dos perfis radiais de velocidade vertical de fluxo do plasma medidos através de técnicas espectroscópicas, vide seção 3.. A Figura 6.11 mostra um exemplo deste ajuste senoidal para um valor de bias de $-25 \mathrm{~V}$. 
Nos resultados apresentados neste capítulo as grandezas estimadas estão dentro das seguintes faixas de valores: $-89 \mathrm{~m}<L_{n}<21 \mathrm{~m},-1,52 \mathrm{~m}^{-1}<k_{z}<0,73 \mathrm{~m}^{-1} \mathrm{e}$ $6,2 \mathrm{~m}^{-1}<q<13,35 \mathrm{~m}^{-1}$.

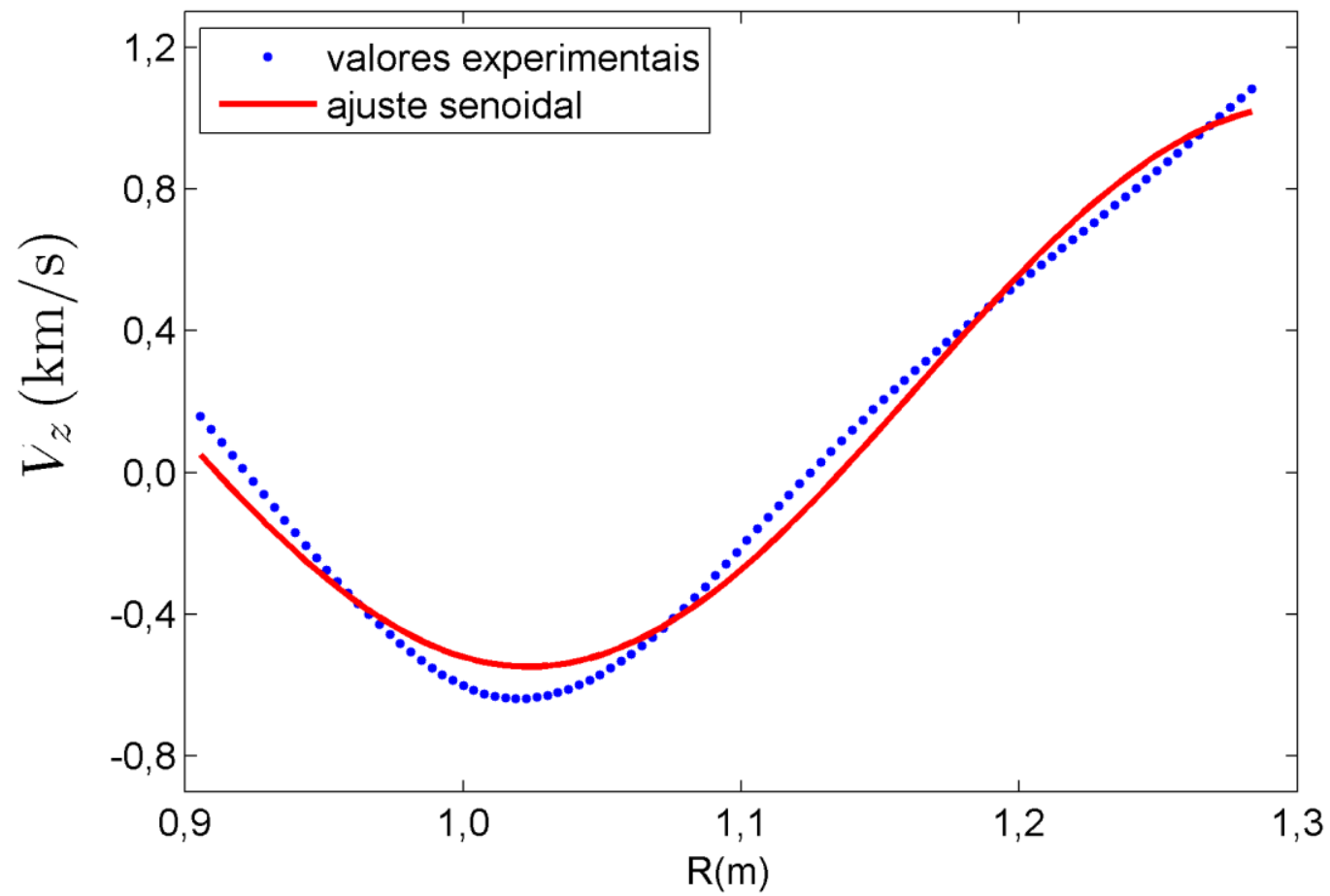

Figura 6.11: Ajuste senoidal aproximado do perfil de velocidade de fluxo vertical, $V_{z}$, para obtenção do número de onda do zonal flow, $q$, necessário para o modelo de quatro ondas acopladas.

Integrando as equações 6.9 - 6.12 (através de um algoritmo de Runge-Kutta de $4^{\mathrm{a}}$ ordem) com os parâmetros obtidos através dos dados experimentais, observa-se que a onda principal, o fluxo do plasma (zonal flow) e as ondas de banda lateral trocam energia entre si e durante este processo a amplitude das ondas é modulada. Para interpretar a largura espectral da turbulência, o tempo de interação, considerado como sendo a metade do período da modulação, foi calculado para cada conjunto de parâmetros experimentais de entrada.

A interpretação feita aqui é de que deve existir uma relação inversa entre a largura espectral, $\Delta f$, e o tempo de interação entre as ondas $\left(T_{\text {inter }}\right)$. De modo que o espectro da turbulência deve ser largo nos casos em que o tempo de interação é curto. Isto porque, um tempo de interação curto significa que, durante a propagação da onda principal, há tempo suficiente para excitar ondas de banda lateral. Estas ondas de banda lateral poderão então assumir o papel de onda principal e, através de uma nova interação 
com o zonal flow, excitar outras ondas de banda lateral. O resultado final deste processo, no qual o tempo de interação é pequeno, será a excitação de muitos modos, levando então a um espectro largo da turbulência.

Para ilustrar a diferença no tempo de interação para dois conjuntos de parâmetros experimentais distintos, a Figura 6.12 mostra a evolução temporal de $a_{+}$ (amplitude de uma das ondas de banda lateral) para duas posições radiais diferentes: $\mathrm{R}$ $=1,110 \mathrm{~m}$ (a) e $\mathrm{R}=1,210 \mathrm{~m}$ (b). Nesta figura, ambas as curvas foram calculadas para $\mathrm{o}$ valor de bias de $-25 \mathrm{~V}$ e para a posição vertical de $\mathrm{z}=1,747 \mathrm{~m}$.
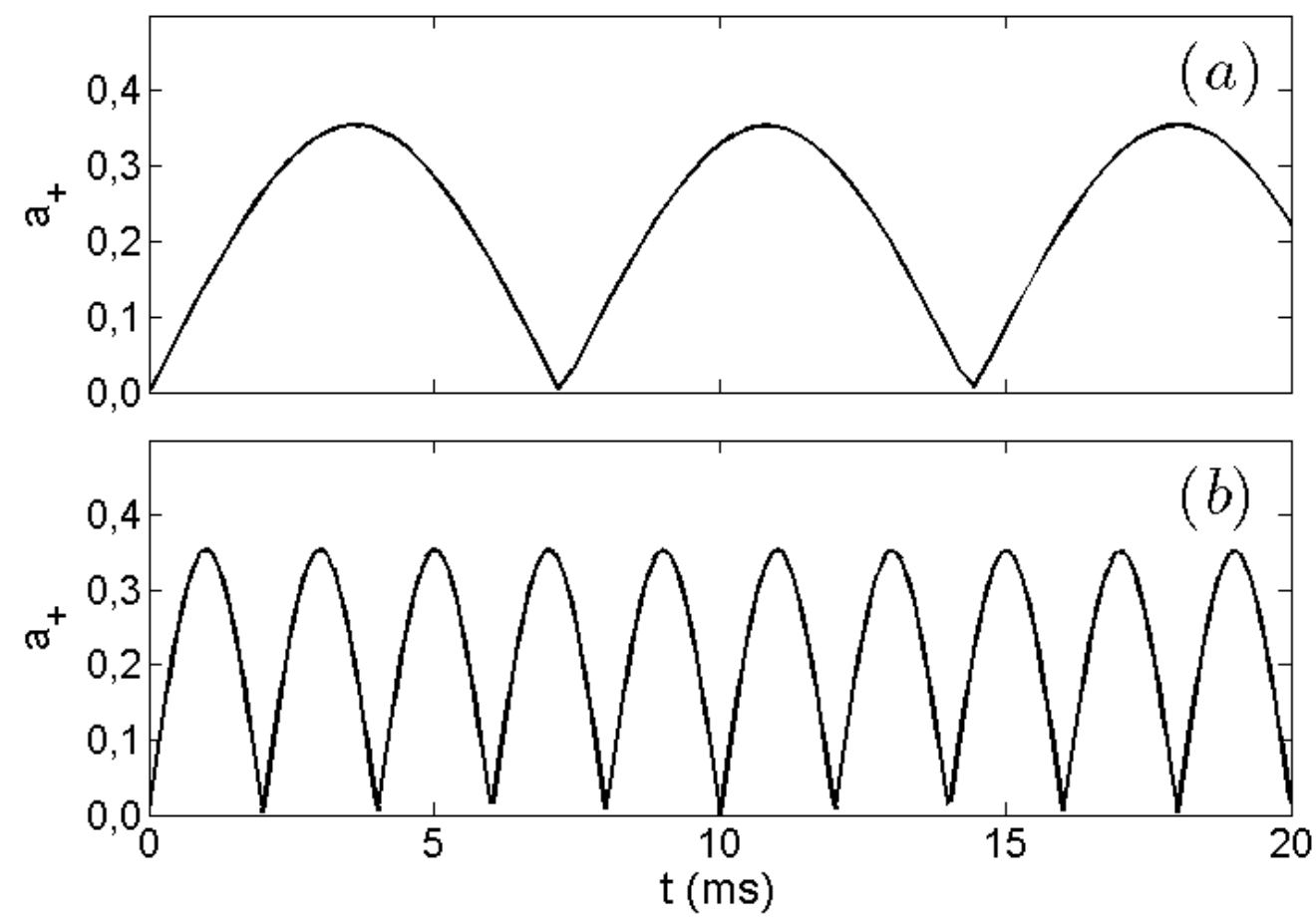

Figura 6.12: Dependência do tempo de interação com as condições do plasma: Evolução temporal da amplitude de uma das ondas de banda lateral $\left(\mathrm{a}_{+}\right)$para duas posições radiais diferentes : (a) $\mathrm{R}=1,110 \mathrm{~m}$ e (b) $\mathrm{R}=1,201 \mathrm{~m}$. Em ambos os casos a posição vertical da sonda $(\mathrm{z}=1,747 \mathrm{~m})$ e o valor do bias ($25 \mathrm{~V})$ são os mesmos.

O tempo médio de interação entre as ondas foi estimado como sendo o tempo de crescimento de $a_{+}$, ou seja, a metade do período da oscilação de $a_{+}$. O tempo de interação estimado em função da posição radial e do valor do bias é visto na Figura 6.13 (a). Nesta figura se observa que o tempo de interação apresenta uma região de valores altos $($ de $R \cong 1,160 \mathrm{~m}$ e bias $\cong-8 \mathrm{~V}$ até $R \cong 1,210 \mathrm{~m}$ e bias $\cong-22 \mathrm{~V})$. Esta região é semelhante à região em que a largura espectral, vista na Figura 6.13 (b), tem valores menores. A concordância entre essas regiões é um indício de que o comportamento da 
turbulência como função do controle externo (bias) pode ser interpretado através do modelo de acoplamento de ondas adotado.

Nota-se por fim, na Figura 6.13, que o modelo utilizado não descreve o comportamento da largura espectral para bias $\cong-16 \mathrm{~V}$ e $\mathrm{R}<1,2 \mathrm{~m}$. Isso ocorre pois o modelo não considera o modo de grande amplitude e baixa frequência, presente nestas condições. Esse modo já foi discutido na seção 5.2.2, Figura 5.10, e será analisado na seção seguinte do ponto de vista do seu acoplamento com outras frequências.
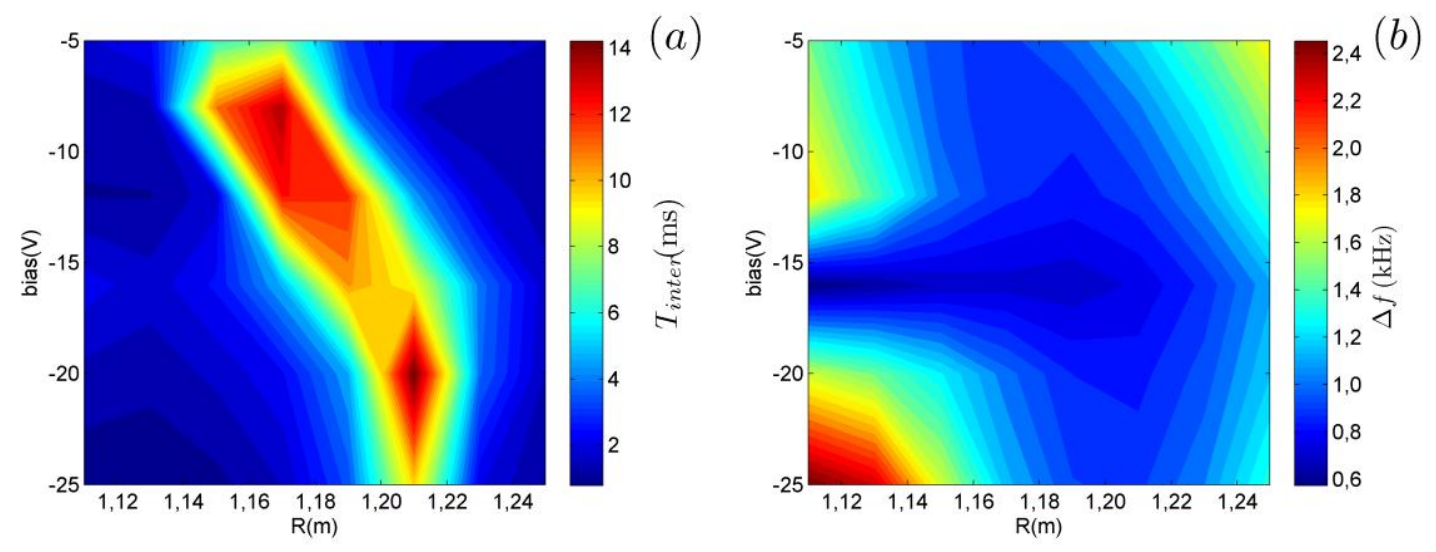

Figura 6.13: (a) Tempo de interação médio e (b) largura espectral média, $\Delta f$, dos sinais de corrente de saturação iônica como função da posição radial e do valor de bias.

\subsection{Análise do modo presente para bias em torno de -16V.}

Para finalizar a análise apresentada neste capítulo, será analisado o comportamento do plasma para bias em torno de $-16 \mathrm{~V}$. Nestes casos, como já comentado, observa-se que o espectro apresenta uma largura muito reduzida o que indica a presença de um modo dominante. A Figura 6.14 (a) mostra o espectro de potência da corrente de saturação iônica para um caso em que o modo de baixa frequência é excitado. Já na Figura 6.14 (b) o espectro de auto-bicoerência para o mesmo caso é mostrado. Observando esse espectro, nota-se que, diferente dos demais casos, esse modo domina o espectro da turbulência e apresenta valores mais elevados de auto-bicoerência para todas as frequências, indicando que este modo dominante apresenta maior acoplamento com toda a faixa de frequências do sinal analisado. 

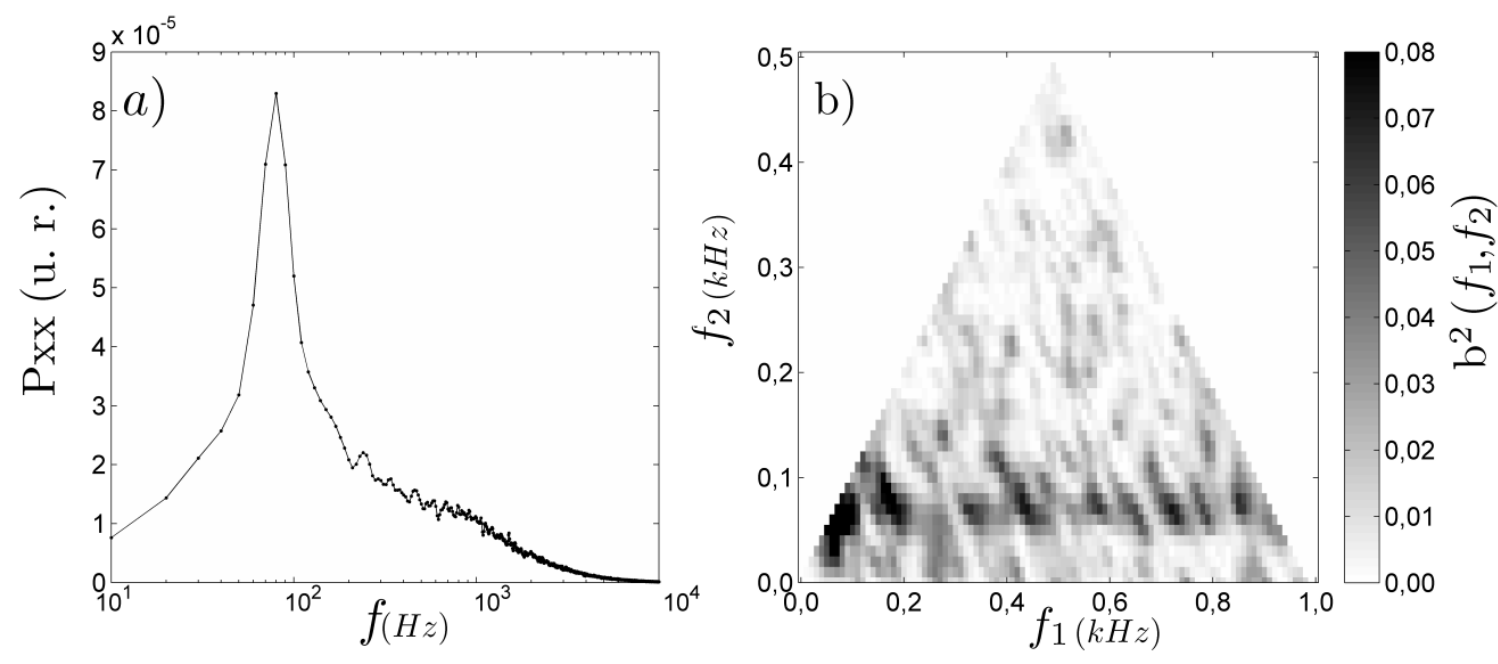

Figura 6.14: (a) Espectro de potência e (b) auto-bicoerência, mostrando o modo de $f=100 \mathrm{~Hz}$. Nota-se que este modo se acopla com todas as freqüências. Estes espectros correspondem a um disparo com bias $=-16 \mathrm{~V}$ e uma sonda posicionada em $(\mathrm{R}=1,210 \mathrm{~m} \mathrm{e} \mathrm{z}=1,747 \mathrm{~m})$.

O acoplamento deste modo de baixa frequência com muitos modos de diversas frequências, observado no espectro de auto-bicoerência (Figura 6.14 (b)), difere do observado para outros valores de bias e de posição radial (Figura 6.9) e pode explicar porque o modelo de quatro ondas acopladas não pode ser utilizado para interpretar a dependência da largura espectral com a posição radial nestes casos. Confirmando isso, a Figura 6.15 mostra o perfil radial da bicoerência soma (a) e da largura espectral (b), tomando-se a média nas diversas sondas posicionadas em diferentes posições verticais. Nesta figura, comparando as dependências de $S_{b}$ e de $\Delta f$ com a posição radial nota-se que estas têm comportamentos opostos (enquanto $S_{b}$ aumenta, $\Delta f$ diminui com o raio), diferente do observado para outros valores negativos de bias (Figura 6.10). Isso mostra que, nos disparos em que esse modo de baixa frequência é observado, bias $\cong-16 \mathrm{~V}$, a largura espectral não pode ser relacionada com o acoplamentos de ondas. 

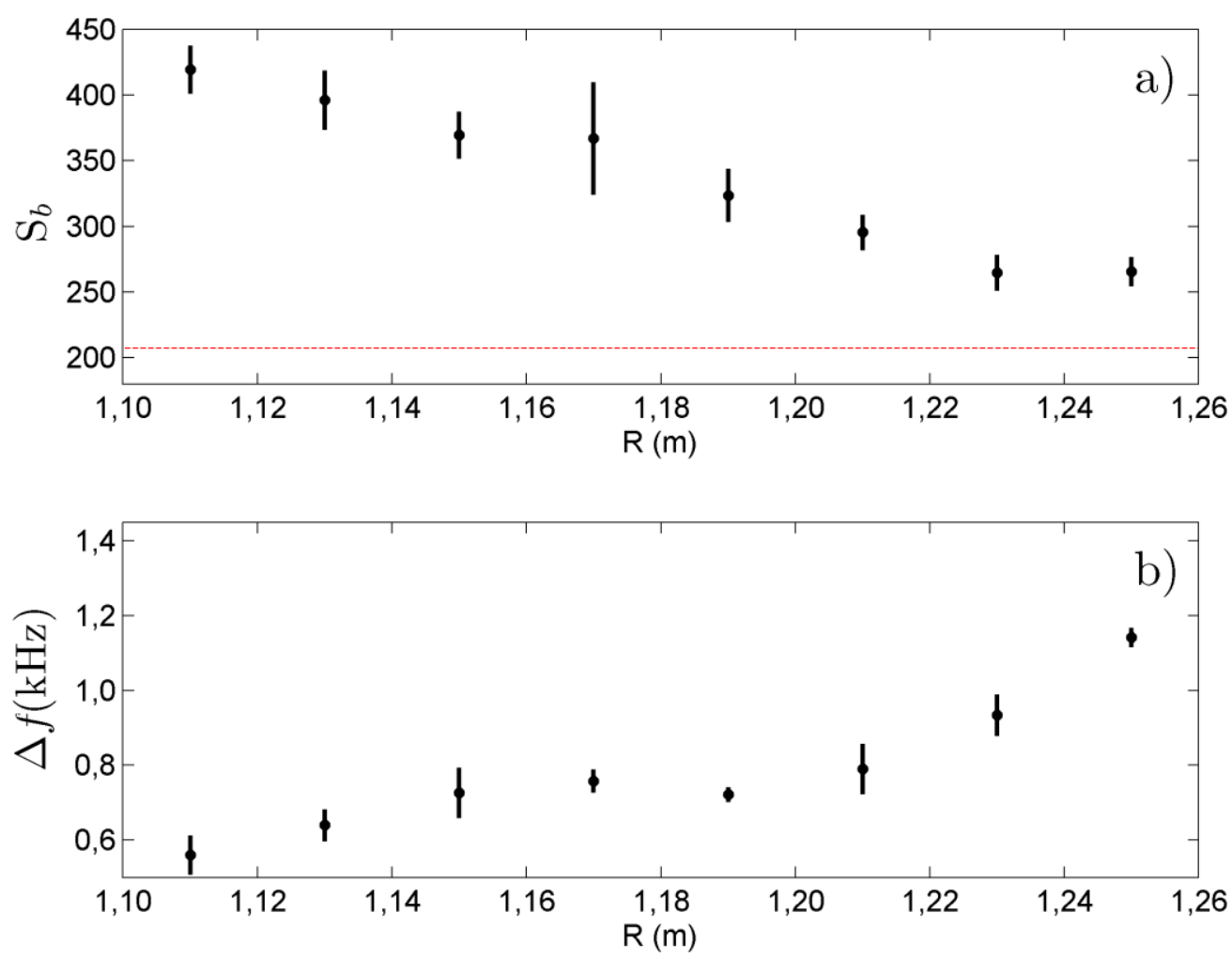

Figura 6.15: Média dos valores verticais da (a) auto-bicoerência soma, $S_{b}$, e (b) largura espectral como função da posição radial para uma descarga com bias $=-16 \mathrm{~V}$. A incerteza de cada ponto foi estimada através do desvio padrão dos valores obtidos no conjunto de seis sondas distribuídas verticalmente. A linha tracejada vermelha indica a soma da auto-bicoerência para um sinal randômico.

\subsection{Considerações finais acerca das alterações da turbulência provocadas pelo bias externo}

Neste capítulo foram estudadas as variações da turbulência presente no Texas Helimak quando da variação do perfil radial do campo elétrico radial. O perfil desse campo é controlado externamente pela imposição de diferentes valores de bias. Para esse estudo foram utilizados os sinais de corrente de saturação iônica medidos por um grande conjunto de sondas de Langmuir distribuídas radial e verticalmente dentro da região radial de interesse $(1,10 \mathrm{~m}<\mathrm{R}<1,25 \mathrm{~m})$. Além desses dados, foram utilizadas medidas espectroscópicas da velocidade vertical do fluxo de plasma.

Conforme conjecturado em outras máquinas do mesmo tipo (FASOLI, 2006), a turbulência eletrostática no Texas Helimak deve estar ligada a instabilidades de deriva (PEREZ, 2006) e de interchange, que são provocadas pelo gradiente da densidade de 
equilíbrio do plasma, e no caso da interchange, também pela curvatura do campo magnético. Estas instabilidades existem separadamente ou coexistem no plasma, dependendo dos parâmetros do plasma, como a intensidade do campo magnético vertical, ou ainda da posição radial medida (POLI, 2007).

Análise espectral e de recorrência foram utilizadas para mostrar que o sinal do bias aplicado modifica de forma distinta as características da turbulência. Assim a turbulência no Texas Helimak foi divida em dois tipos, de acordo com o sinal do bias aplicado.

Descargas tomadas com bias positivo apresentaram maior potência espectral total, espectro de banda larga, cuja largura aumenta com o valor do bias, e valores menores de determinismo. Através das funções de distribuição de probabilidade foi observado que esse tipo de turbulência é marcado pela presença de eventos extremos. Por outro lado, descargas com bias negativo apresentaram uma largura espectral, $\Delta f$, menor e cuja dependência com o valor do bias não é direta. A análise de recorrência mostrou que o determinismo nesta situação é maior em relação à turbulência com bias positivo.

Através de análise biespectral foi mostrado que, para descargas com bias negativo, a largura espectral está relacionada com o acoplamento de modos, uma vez que valores altos de $\Delta f$ coincidem com valores altos de auto-bicoerência soma, $S_{b}$. Assim, um modelo de quatro ondas acopladas, que inclui o fluxo do plasma (zonal flow), foi adotado para interpretar a dependência do $\Delta f$ com o valor do bias e da posição radial. Usando parâmetros experimentais nesse modelo, o tempo de interação ( $T_{\text {inter }}$ ) foi calculado e comparado com os valores experimentais de $\Delta f$. O resultado desta comparação indica que para maiores valores de $T_{\text {inter }}$, poucos modos de banda lateral devem ser excitados e, como consequência, a largura espectral é pequena. (TOUFEN, 2012-2). 


\section{CONTROLE DO TRANSPORTE RADIAL DE PARTÍCULAS}

No Texas Helimak o perfil radial do campo elétrico pode ser alterado através da imposição de potenciais elétricos externos (bias), o que propicia uma maneira de controlar não apenas a turbulência, como já discutido nos capítulos anteriores, mas também o transporte radial de partículas. Esse controle do transporte de partículas no Texas Helimak será objeto de estudo deste capítulo. Como no capítulo anterior, a análise do transporte será separada de acordo com o sinal do bias aplicado.

Neste capítulo será analisado o transporte turbulento de partículas no Texas Helimak, bem como sua dependência com o perfil radial da componente radial do campo elétrico, modificado através da imposição de potencial elétrico externo no plasma (bias). Utilizando as medidas espectroscópicas da velocidade de fluxo vertical do plasma, perfis radiais do cisalhamento (shear) da velocidade foram calculados para verificar a dependência dos perfis de transporte de partículas com a velocidade de fluxo e seu cisalhamento. Um modelo Hamiltoniano de transporte caótico de partículas (MARCUS, 2008-1) foi utilizado para interpretar os perfis radiais do transporte de partículas e sua dependência com o bias (TOUFEN, 2012).

\subsection{Transporte induzido por ressonância}

Os valores de transporte turbulento, $\Gamma$, mostrados neste capítulo foram estimados através da análise descrita na seção 3.3, eq. 3.17. Os dados para essa análise foram obtidos da distribuição de sondas mostrada na Figura 7.1. Nota-se que nesta distribuição existem cinco conjuntos de três sondas cada (duas medindo potencial flutuante e uma corrente de saturação iônica) o que permite a medida de perfis de transporte turbulento com cinco posições radiais distintas. 


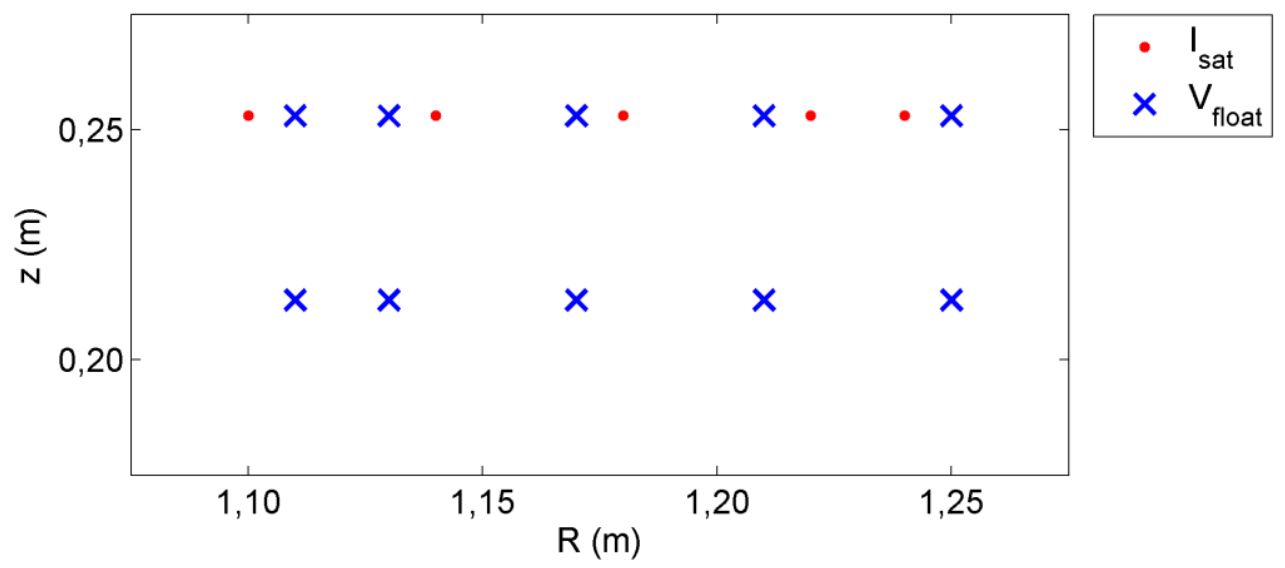

Figura 7.1: Distribuição de sondas utilizada para medidas de transporte turbulento.

Utilizando a distribuição de sondas mostrada na Figura 7.1 e a técnica de análise mencionada, o perfil radial do transporte turbulento de partículas na direção radial foi estimado para bias nulo e demais valores negativos de bias externo. Na Figura 7.2 é apresentado um diagrama com os perfis radiais do transporte para valores de bias de $-30 \mathrm{~V}$ à zero. Nesta figura o transporte é mostrado em unidades relativas sendo que as regiões em vermelho representam o transporte positivo (no sentido crescente da coordenada R) e as regiões em azul o transporte negativo (para o centro da máquina). Observa-se na Figura 7.2 que o transporte depende tanto da posição radial quanto do valor de bias. Neste diagrama, há uma região com valores grandes do transporte de $\mathrm{R}=$ $1,11 \mathrm{~m}$ e bias $=-8 \mathrm{~V}$ até $\mathrm{R}=1,14 \mathrm{~m}$ para bias $\cong-25 \mathrm{~V}$. 


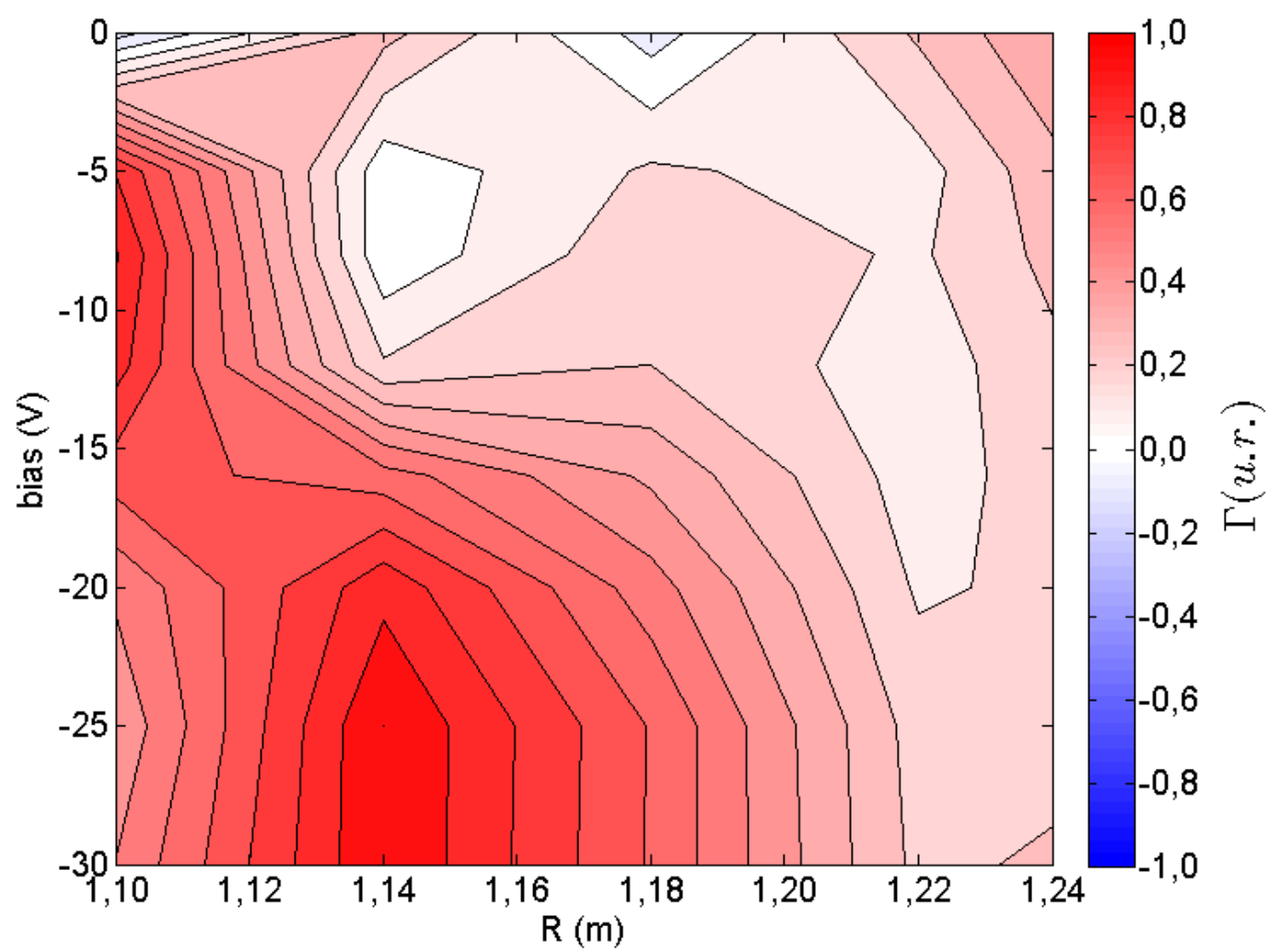

Figura 7.2: Perfis radiais do transporte turbulento de partículas, $\Gamma$, em função do bias, para valores negativos de bias.

O bias imposto externamente altera não só o transporte estimado de partículas, mas também a velocidade vertical do fluxo de plasma, que no Helimak pode ser medida através do deslocamento Doppler das linhas de emissão do Argônio, ver Figura 5.15. Utilizando as medidas de velocidade do fluxo foram calculados os perfis radiais de cisalhamento (shear), $d V_{z} / d R$, como função do bias, de modo que se possa analisar a relação entre o transporte de partículas e o cisalhamento. Estes perfis radiais de cisalhamento são vistos na Figura 7.3. Nesta figura observa-se que o cisalhamento apresenta valores maiores para valores mais negativos de bias, especialmente na região para bias $<-20 \mathrm{~V}$ e $\mathrm{R}<1,18 \mathrm{~m}$.

A influência da velocidade de fluxo com cisalhamento sobre o transporte é prevista em (BIGLARI, 1990) e (TERRY, 2000). Segundo estas previsões, um valor elevado de cisalhamento rompe as estruturas macroscópicas do plasma e, com isso, reduz o transporte anômalo de partículas nesta região. Comparando a Figura 7.2 com a Figura 7.3 observa-se que, contrariamente à previsão, o transporte é elevado em regiões com cisalhamento elevado, especialmente na região em que bias $\cong-25 \mathrm{~V}$ e $\mathrm{R}=1,14 \mathrm{~m}$. 
Assim, a falta de redução de transporte indica que, ao contrário da previsão teórica mencionada, na região analisada do Helimak Texas o aumento do cisalhamento da velocidade não reduz o transporte turbulento.

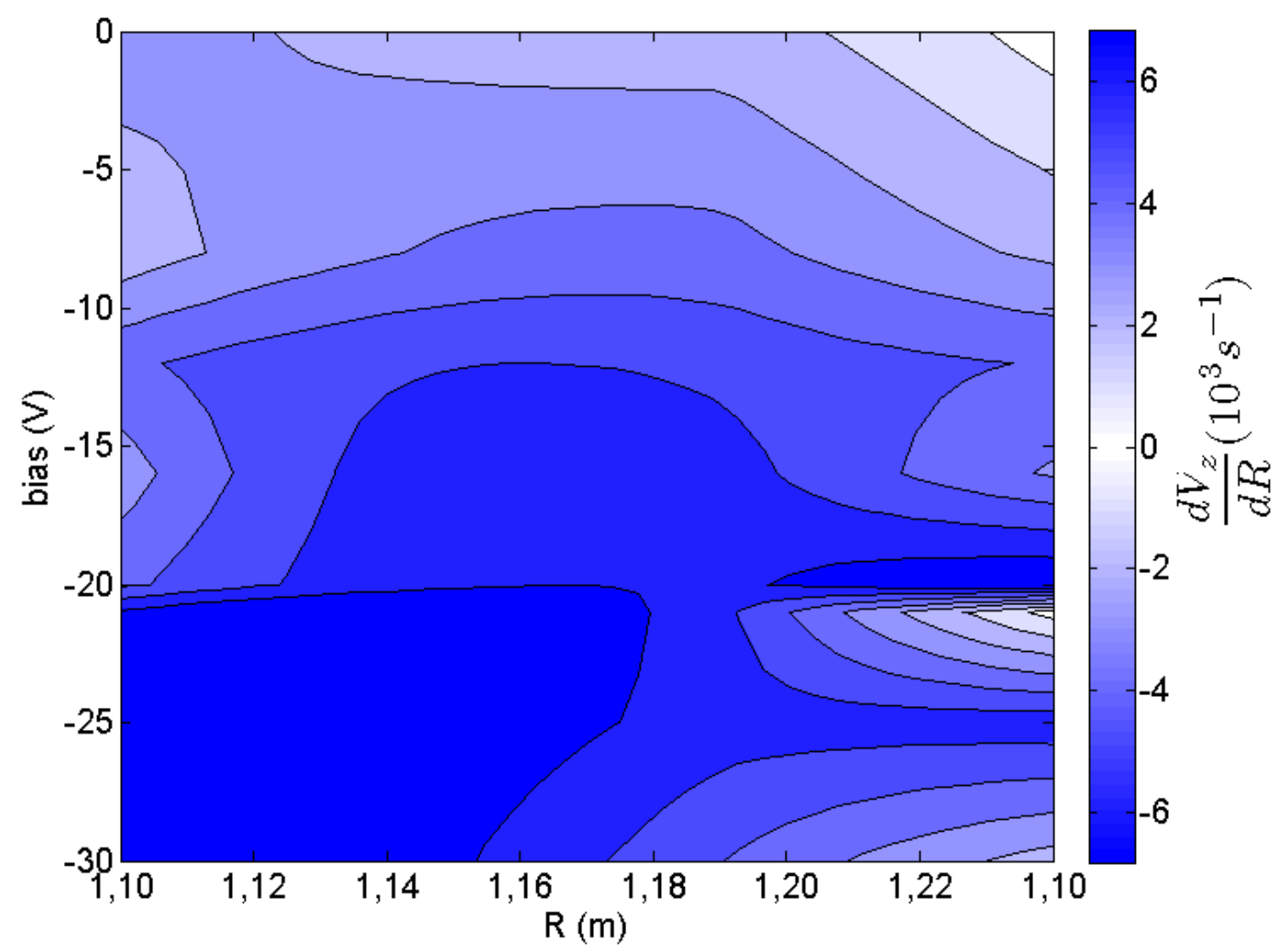

Figura 7.3: Perfis radiais do Cisalhamento (shear) da velocidade vertical do fluxo de plasma para valores negativos de bias.

\subsubsection{Interpretação teórica: transporte caótico na ressonância}

Uma vez que a redução do transporte para valores grandes de cisalhamento, prevista teoricamente (TERRY, 2000), não foi verificada para valores negativos de bias, um modelo teórico alternativo da dependência do transporte com o perfil do campo elétrico, que é modificado pelo bias externo, será utilizado.

Uma possível interpretação, baseada no transporte caótico, para a dependência do transporte de partículas com o perfil do campo elétrico radial é dada em (MARCUS, 2004). Neste trabalho o autor supõe que a velocidade do fluxo na direção poloidal seja dada principalmente pela velocidade de deriva $\mathrm{E} \times \mathrm{B}$. Para um campo magnético constante na direção toroidal $\left(\mathbf{B}=B_{0} \hat{e}_{T}\right)$ e um potencial elétrico $(\phi)$ dado por um 
potencial eletrostático de equilíbrio $\left(\phi_{0}(x)\right)$ perturbado por duas ondas de deriva (lembrando que: $\mathbf{E}=-\nabla \phi$ ), o movimento de uma partícula carregada pode ser obtido por uma Hamiltoniana (MARCUS, 2008-1) dada por:

$$
\begin{aligned}
H(x, y, t)= & \phi_{0}(x)-u_{1} x+A_{1} \operatorname{sen}\left(k_{x 1} x\right) \cos \left(k_{y 1} y\right)+ \\
& A_{2} \operatorname{sen}\left(k_{x 2} x\right) \cos \left[k_{y 2}(y-u t)\right],
\end{aligned}
$$

sendo $x$ e $y$ as coordenadas radial e vertical normalizadas, $t$ o tempo normalizado, $u$ a diferença entre as velocidades de fase das duas ondas, e $u_{1}$ a velocidade de fase da primeira onda, dados por:

$$
\begin{aligned}
& x=\frac{R}{a}, \\
& y=\frac{z}{a}, \\
& t=\frac{t^{\prime}}{a} E_{0}, \\
& u=\frac{\omega_{2}}{k_{y 2}}-\frac{\omega_{1}}{k_{y 1}}, \\
& u_{1}=\frac{\omega_{1}}{k_{y 1}},
\end{aligned}
$$

sendo $a$ o raio médio do Texas Helimak e $E_{0}$ o campo elétrico radial de equilíbrio.

No caso em que se despreza uma das ondas de deriva $\left(A_{2}=0\right)$, a Hamiltoniana do sistema passa a ser integrável e as partículas ficam confinadas em curvas com $H_{1}$ constante:

$$
H_{1}(x, y)=\phi_{0}(x)-u_{1} x+A_{1} \operatorname{sen}\left(k_{x 1} x\right) \cos \left(k_{y 1} y\right)
$$

Para o caso em que o campo elétrico é uniforme, as equações de movimento podem ser escritas da seguinte forma:

$$
\begin{aligned}
& \frac{d X}{d T}=\operatorname{sen}(X) \operatorname{sen}(Y), \\
& \frac{d Y}{d T}=U(X)+\cos (X) \cos (Y),
\end{aligned}
$$


onde foi realizada a seguinte mudança de variáveis: $X=k_{x 1} x, Y=k_{y 1} y$ e $T=$ $A_{1} k_{x 1} k_{y 1}$ e sendo $A_{1}$ a amplitude da onda, $k_{x 1}$ o número de onda na direção radial e $k_{y 1}$ o número de onda na direção vertical.

Este sistema possui apenas um parâmetro adimensional relevante, o parâmetro de confinamento, $U$, dado por:

$$
U(X)=U(x)=\frac{1}{A_{1} k_{x 1}}\left[\frac{d \phi_{0}(x)}{d x}-u_{1} B_{0}\right]=\frac{B_{0}}{A_{1} k_{x 1}}\left[V_{E}(x)-u_{1}\right],
$$

sendo $V_{E}$ a velocidade de deriva eletrostática:

$$
V_{E}(x) \mathbf{e}_{z}=\frac{E \mathbf{e}_{r} \times B_{0} \mathbf{e}_{T}}{B_{0}^{2}} .
$$

Resultados numéricos obtidos com o uso desse modelo mostram que o espaço de fase do sistema fica preenchido por ilhas, Figura 7.4 (a), quando o parâmetro $U$ é zero. Para $U=0$ há ressonância, uma vez que as velocidades de fase da onda e a velocidade de deriva do plasma são iguais. Entre as ilhas existem separatrizes transversais as quais, quando o sistema é perturbado, Figura 7.5 (a), são quebradas dando origem a um transporte grande de partículas. A medida que o parâmetro $U$ aumenta, surgem barreiras no espaço de fase, Figura 7.4 (b), que reduzem o transporte de partículas na direção radial no caso do sistema perturbado, Figura 7.5 (b). O sistema representado nesta figura assume campo elétrico uniforme. Para $U>1$ não há formação de ilhas no espaço de fase.
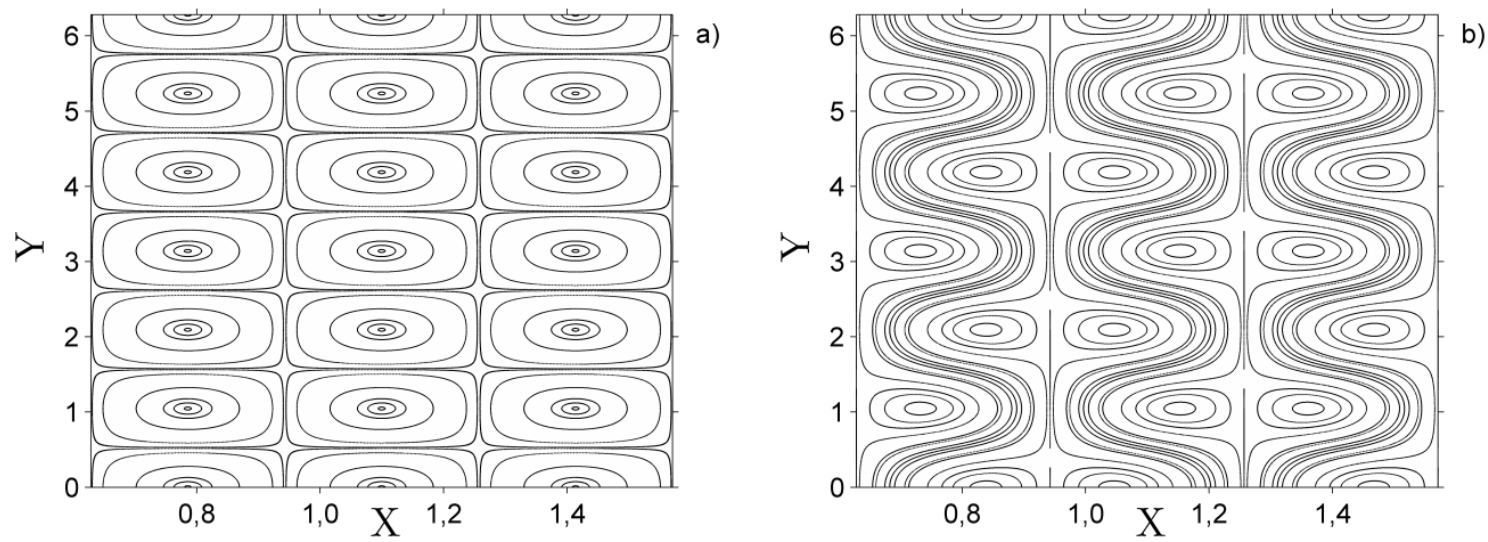

Figura 7.4: Espaços de fase do sistema integrável $\left(A_{2}=0\right)$ de uma onda, eq. 7.7, para $\mathrm{U}=0$ (a) e $\mathrm{U}=0,5$ (b). Nestes espaços de fase $X$ e $Y$ estão ligados, respectivamente, as coordenadas radial e vertical. 

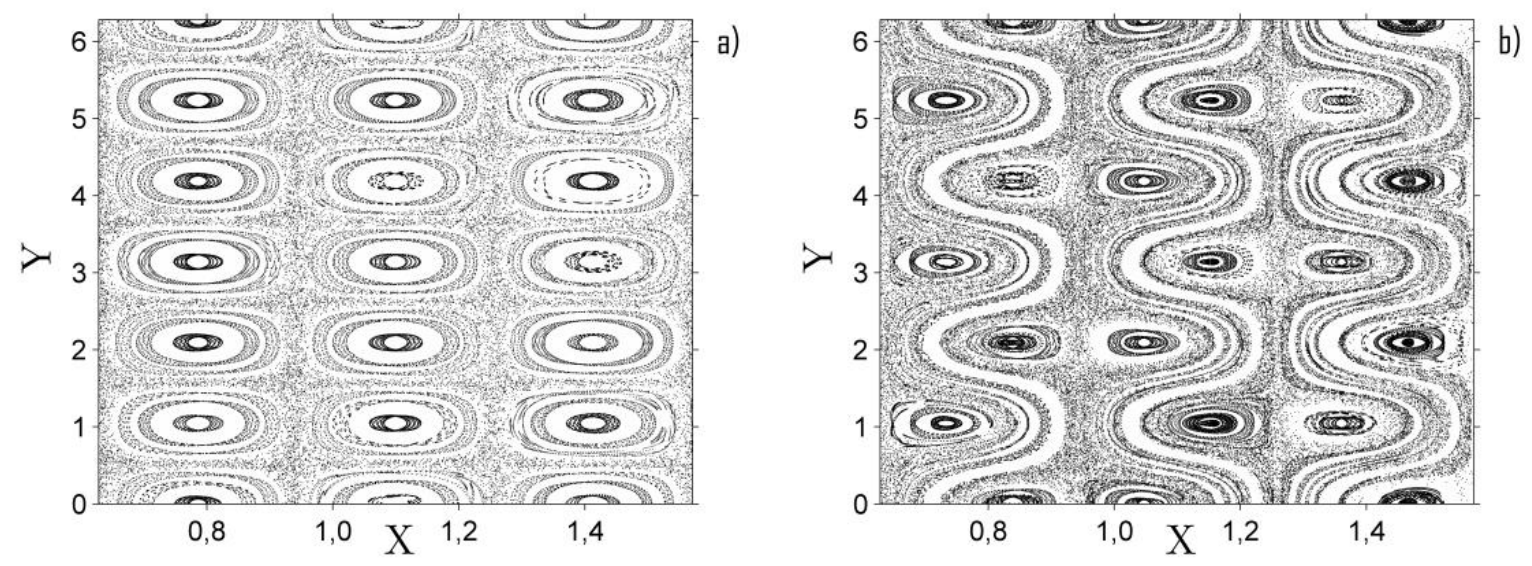

Figura 7.5: Mapa estroboscópico do sistema de duas ondas, eq. 7.1, para $\mathrm{U}=0$ (a) e $\mathrm{U}=0,5$ (b). A relação da amplitudes das ondas nestes mapas é de $A_{2} / A_{1}=0,01$. Nestes espaços de fase $X$ e $Y$ estão ligados, respectivamente, as coordenadas radial e vertical.

Nesse modelo, portanto, o transporte radial depende da relação entre a amplitude relativa da perturbação, $A_{2} / A_{1}$, que caso seja muito pequena irá reduzir o transporte e o valor do parâmetro de confinamento $U$, que, caso seja muito diferente de zero (detunin) também irá reduz o transporte devido a formação de barreiras.

Para interpretar a dependência do transporte de partículas com o bias através deste modelo Hamiltoniano, a diferença de velocidades $V_{E}-V_{p h}$, que é diretamente proporcional ao parâmetro de confinamento $U$, foi calculada como função da posição radial e do valor do bias, sendo o resultado mostrado na Figura 7.6. A previsão do modelo é que para $V_{E}-V_{p h} \cong 0$, e consequentemente $U \cong 0$, o transporte seja elevado (cenário mostrado no espaço de fase da Figura 7.4 (a) ). Neste caso não existem barreiras para o fluxo radial de partículas e o transporte ocorre nas regiões em que a separatriz foi quebrada. Comparando a região mais escura da Figura 7.6, que mostra para que condições $V_{E}-V_{p h} \cong 0$, com as regiões de transporte alto mostradas na Figura 7.2, de cor vermelha escura, verifica-se que de um modo geral ambas coincidem ou estão próximas. Essa concordância indica que os valores altos observados nos perfis de transporte para valores negativos de bias podem ser entendidos como consequência da ressonância entre a velocidade de deriva elétrica, $V_{E}$, e a velocidade de fase da onda que trafega no plasma, $V_{p h}$. 


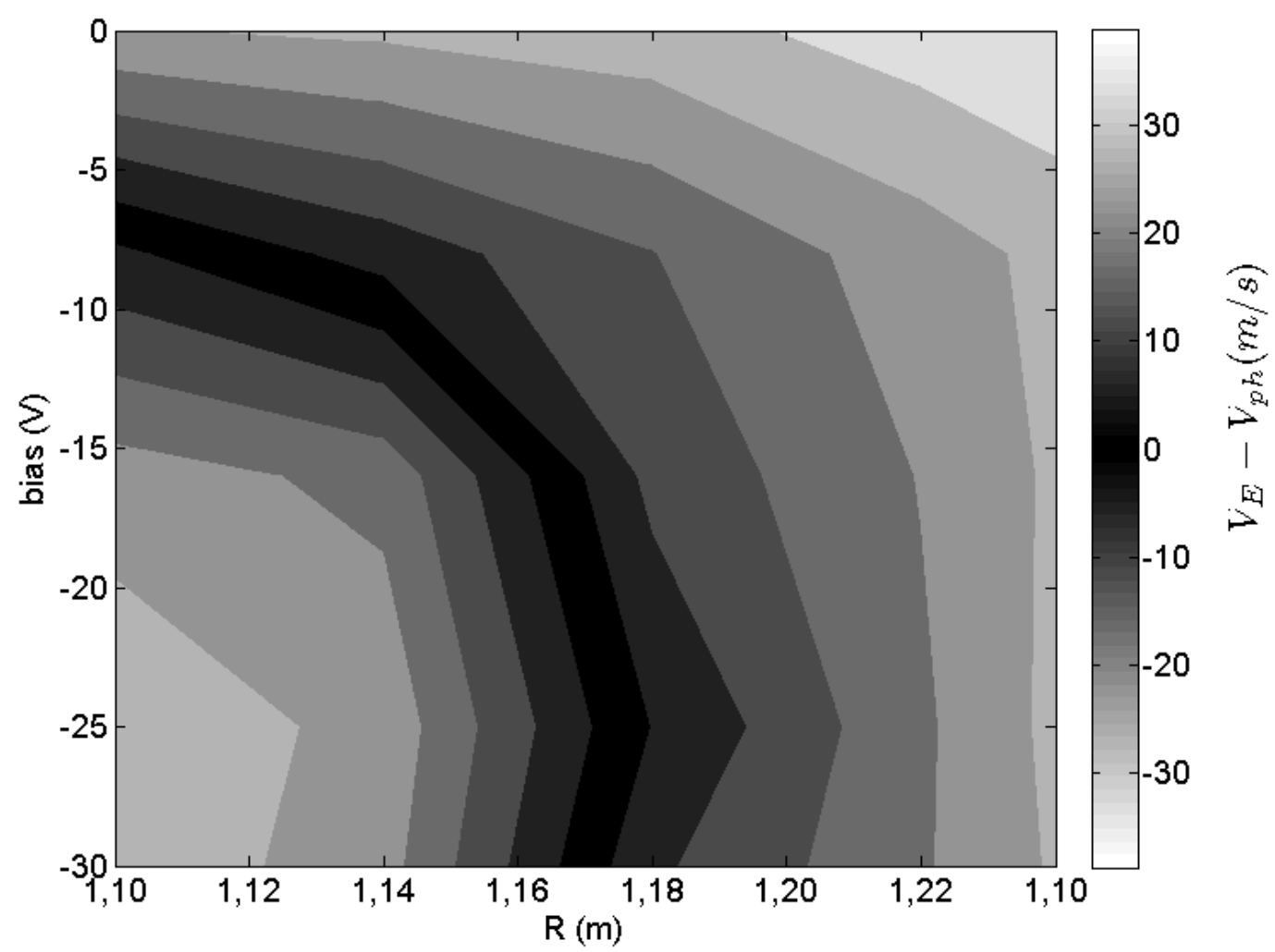

Figura 7.6: Perfis radiais da diferença entre a velocidade de deriva elétrica, $V_{E}$, e a velocidade de fase, $V_{p h}$, em função de valores negativos de bias. Essa diferença é proporcional ao parâmetro de confinamento: $U$.

\subsection{Evidência de barreira de transporte de cisalhamento nulo (shearless)}

Ao contrário dos disparos tomados com valores negativos de bias, discutidos na seção 6.1, os disparos tomados com valores positivos de bias, que serão discutidos nesta seção, apresentam perfis radiais não monotônicos da velocidade vertical de fluxo de plasma na faixa radial de análise $(1,10 \mathrm{~m}<\mathrm{R}<1,25 \mathrm{~m})$. Com isso, para bias positivo, os perfis de velocidade de fluxo apresentam um ponto de máximo dentro da região de análise e, consequentemente, um ponto sem cisalhamento (shearless) pois, no máximo da curva tem-se que: $d V_{z} / d R=0$. A Figura 7.7 (a) mostra um exemplo, para bias $=+10 \mathrm{~V}$, de perfil radial de velocidade de fluxo vertical mostrando o ponto de máximo em $R=R^{*}$, enquanto que a Figura 7.7 (b) mostra o perfil do cisalhamento correspondente. 

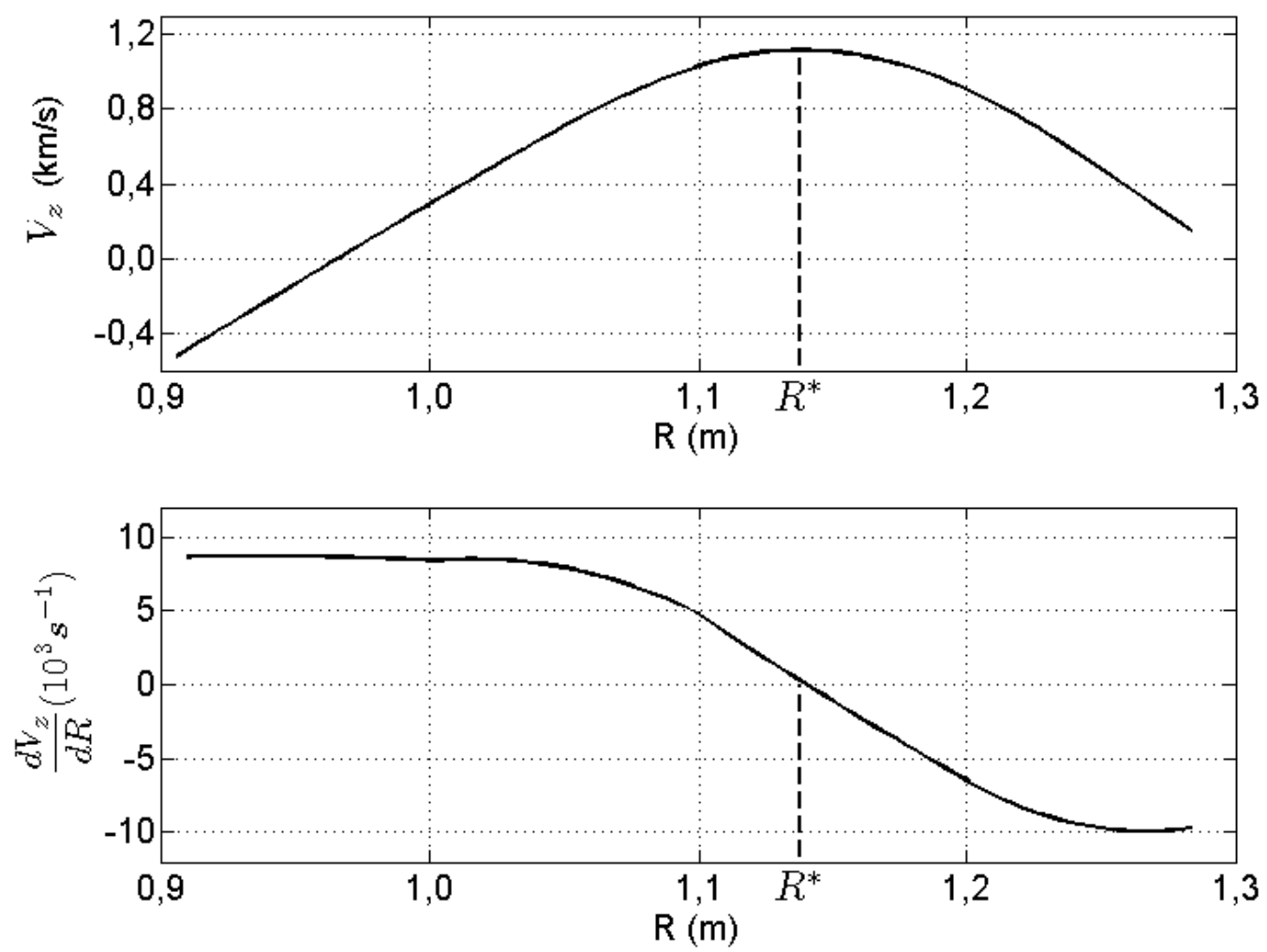

Figura 7.7: Perfil radial da velocidade vertical do plasma (a) e do cisalhamento (b) para bias $=+10 \mathrm{~V}$. Nestas curvas destaca-se a posição radial, $R^{*}$, em que a velocidade apresenta um ponto de máximo e o cisalhamento é zero (ponto sem shear).

O transporte de partículas em fluxos com cisalhamento reverso tem sido amplamente estudado, tanto em fluidos (SOMMERIA, 1989, SOLOMON, 1993 e DELCASTILLO-NEGRETE, 2000) como em plasmas (MARCUS, 2008-2). Estes estudos mostram a presença de uma barreira de transporte na região em que o cisalhamento muda de sinal. Para estudar o efeito da inversão do cisalhamento no transporte turbulento de partículas, perfis radiais do transporte $(\Gamma)$, e do cisalhamento $\left(d V_{z} / d R\right)$, foram calculados como função do raio para descargas com bias positivos, pois são nestas descargas que o cisalhamento apresenta inversão de sinal. Os perfis de transporte são mostrados na Figura 7.8 e os do cisalhamento são mostrados na Figura 7.9. Na Figura 7.8, as regiões em azul indicam valores negativos de transporte, ou seja, o transporte aponta para o centro da máquina, já as regiões em vermelho indicam transporte positivo, apontando para a borda externa do plasma.

Comparando os perfis de transporte com o cisalhamento, observa-se que para todos os valores de bias analisados o transporte de partículas é muito pequeno onde o 
cisalhamento da velocidade é zero. Isto pode ser interpretado como uma indicação da existência de uma barreira de cisalhamento zero (shearless).

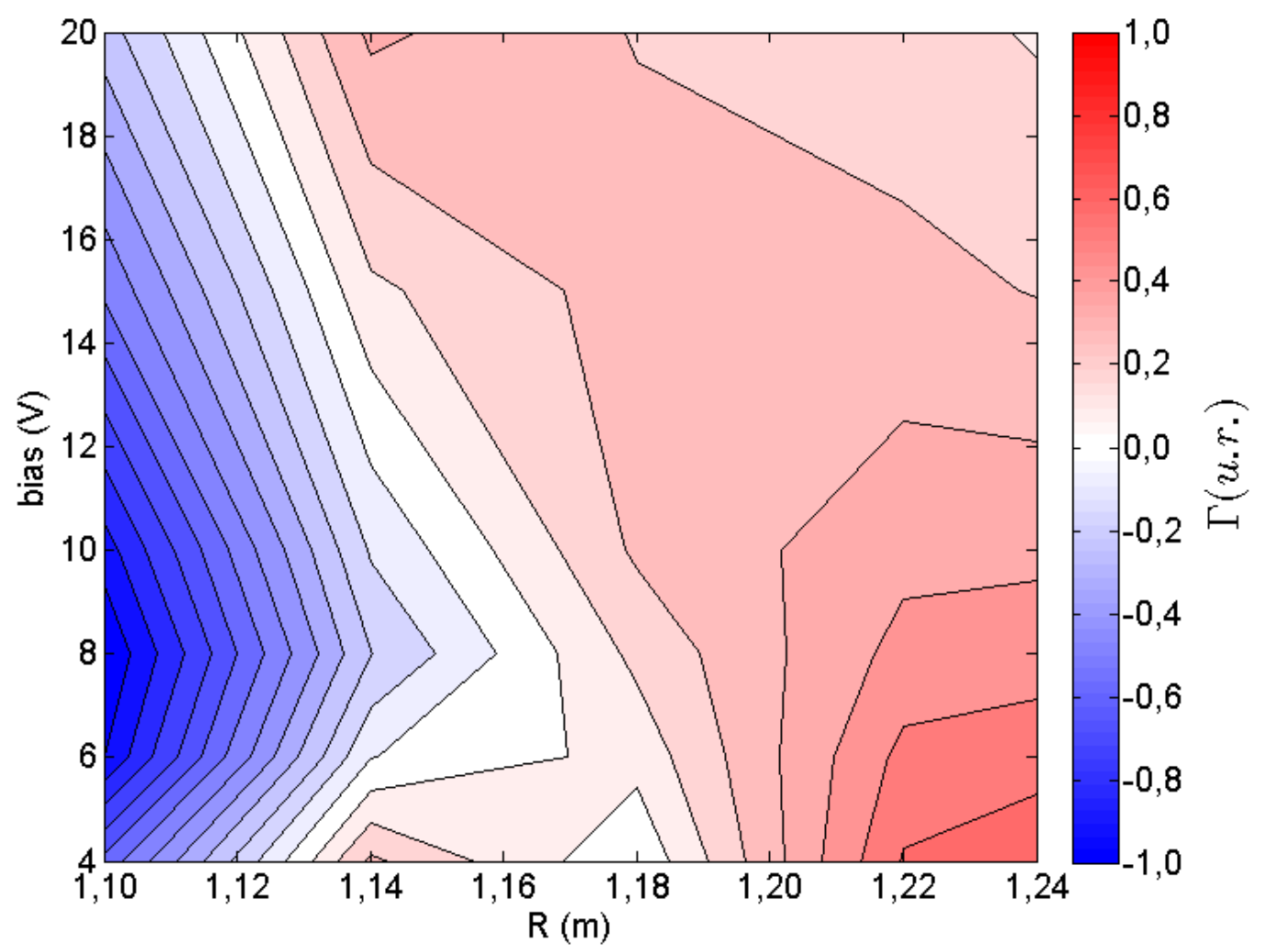

Figura 7.8: Perfis radiais do transporte turbulento de partículas, $\Gamma$, em função do bias, para valores positivos de bias. 


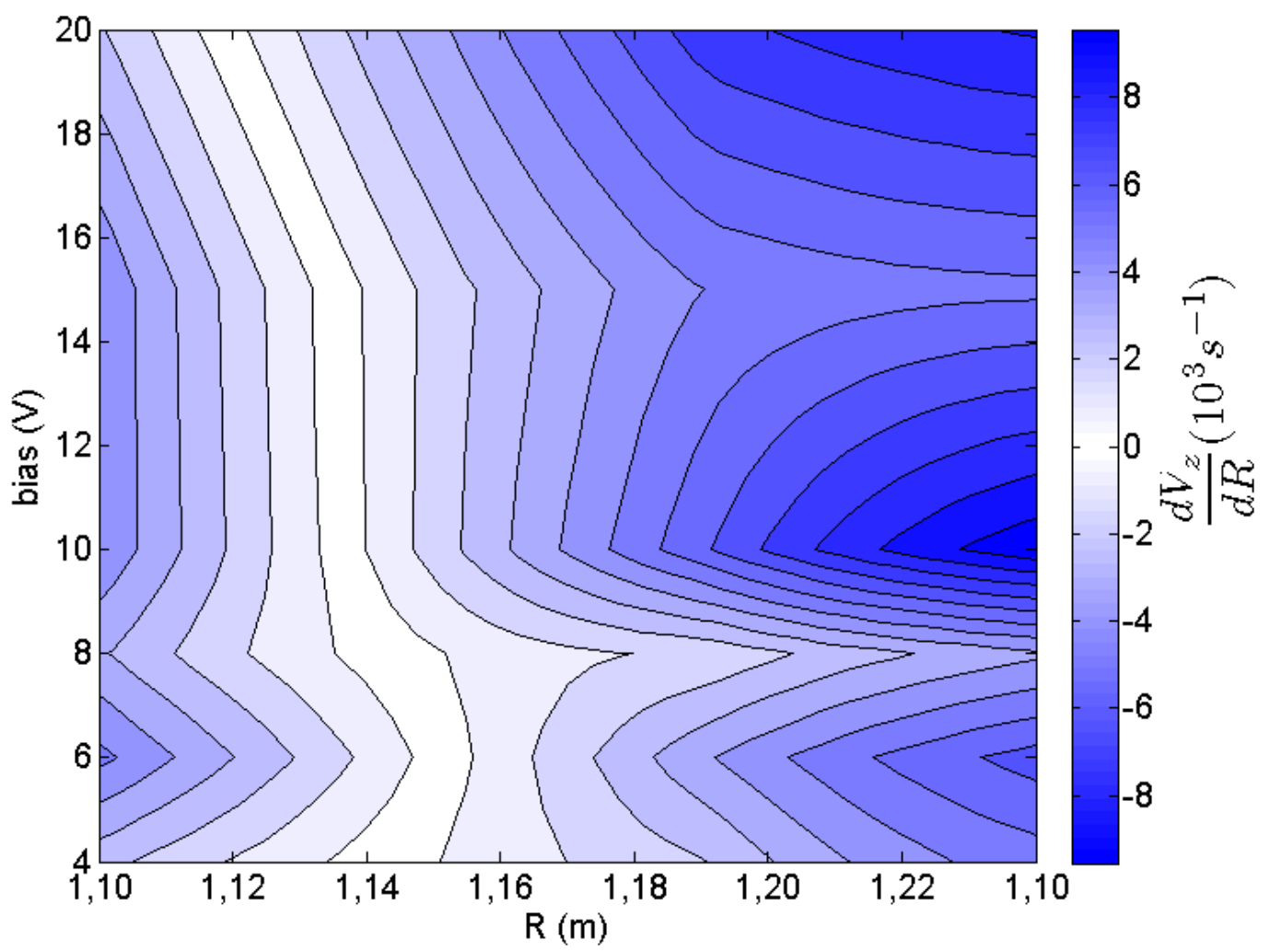

Figura 7.9: Perfis radiais do cisalhamento (shear) da velocidade vertical do fluxo de plasma para valores positivos de bias.

\subsubsection{Interpretação teórica: barreiras de cisalhamento zero devidas aos perfis não monotônicos de $U(x)$}

Para interpretar esse efeito de barreira de transporte de cisalhamento nulo, o modelo Hamiltoniano já apresentado, cuja Hamiltoniana é vista na equação 7.1, foi modificado de modo que o parâmetro de confinamento $U(x)$, equação 7.10, apresente um perfil radial não monotônico.

Este perfil não monotônico de $U(x)$ ocorre devido a sua relação com a velocidade de deriva elétrica, $V_{E}(x)$. Assumindo-se que a velocidade de fluxo do plasma é majoritariamente resultado da deriva elétrica $\left(\boldsymbol{E} \times \boldsymbol{B} / \boldsymbol{B}^{2}\right)$, tem-se que:

$$
V_{E} \cong V_{z}
$$

Como os perfis experimentalmente medidos de $V_{Z}$ são não monotônicos, para os disparos analisados nesta seção, os perfis de $U(x)$ também serão não monotônicos, 
desde que se assuma que a velocidade de fase $u_{1}$ é aproximadamente constante na faixa radial analisada.

Assim, para obter a Hamiltoniana na qual o perfil de $U(x)$ é não monotônico ajustou-se uma parábola ao perfil experimental de $V_{z}$ para bias $=+10 \mathrm{~V}$, ajuste apresentado na Figura 7.10, e assumindo que $V_{E} \cong V_{z}$, o perfil de $\phi_{0}(x)$ pode ser estimado através da equação 7.13 .

$$
V_{E}=\frac{1}{B} \cdot \frac{d \phi_{0}(x)}{d x}
$$

Utilizando o perfil de $\phi_{0}(x)$ encontrado na Hamiltoniana da equação 7.1 e a velocidade de fase média $u_{1}$, calculou-se a seção de Poincaré (KUZNETSOV, 2004, Pg. 26) do centro de guia das órbitas no espaço de fase. A seção de Poincaré obtida é vista na Figura 7.11 (b) sendo que o perfil do parâmetro de confinamento, $U(x)$, correspondente é visto na Figura 7.11 (a).

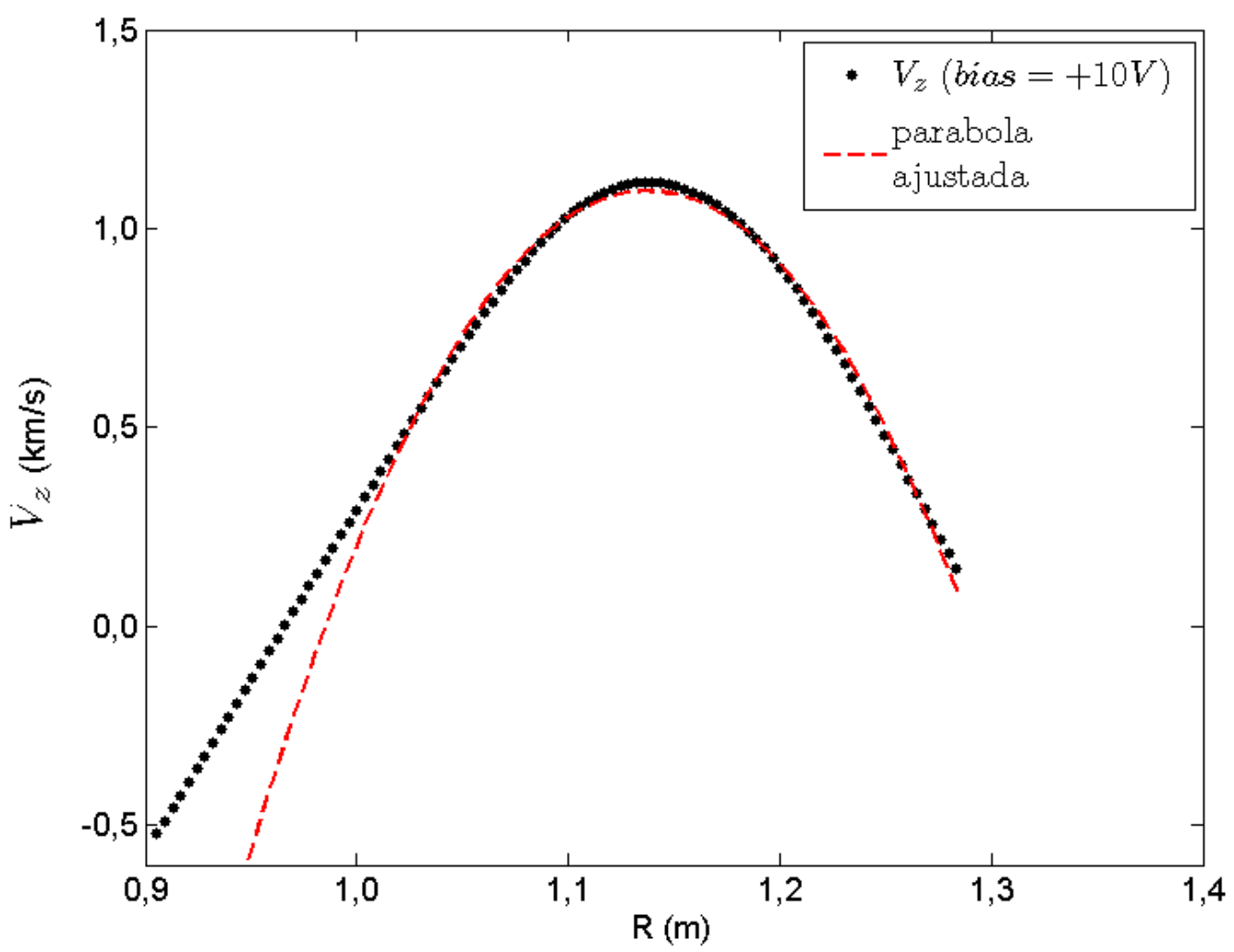

Figura 7.10: Perfil radial da velocidade vertical do plasma (pontos pretos) e parábola ajustada (curva vermelha) para bias $=+10 \mathrm{~V}$. 
A seção de Poincaré mostrada na Figura 7.11 (b) indica a presença de uma barreira de transporte localizada próxima à posição em que o cisalhamento é nulo $(X \cong 1,1)$, onde $U(X)$ apresenta um máximo. Consequentemente, esta barreira pode explicar a redução do transporte de partículas, região branca da Figura 7.8, na posição radial em que o cisalhamento é zero, região branca da Figura 7.9.

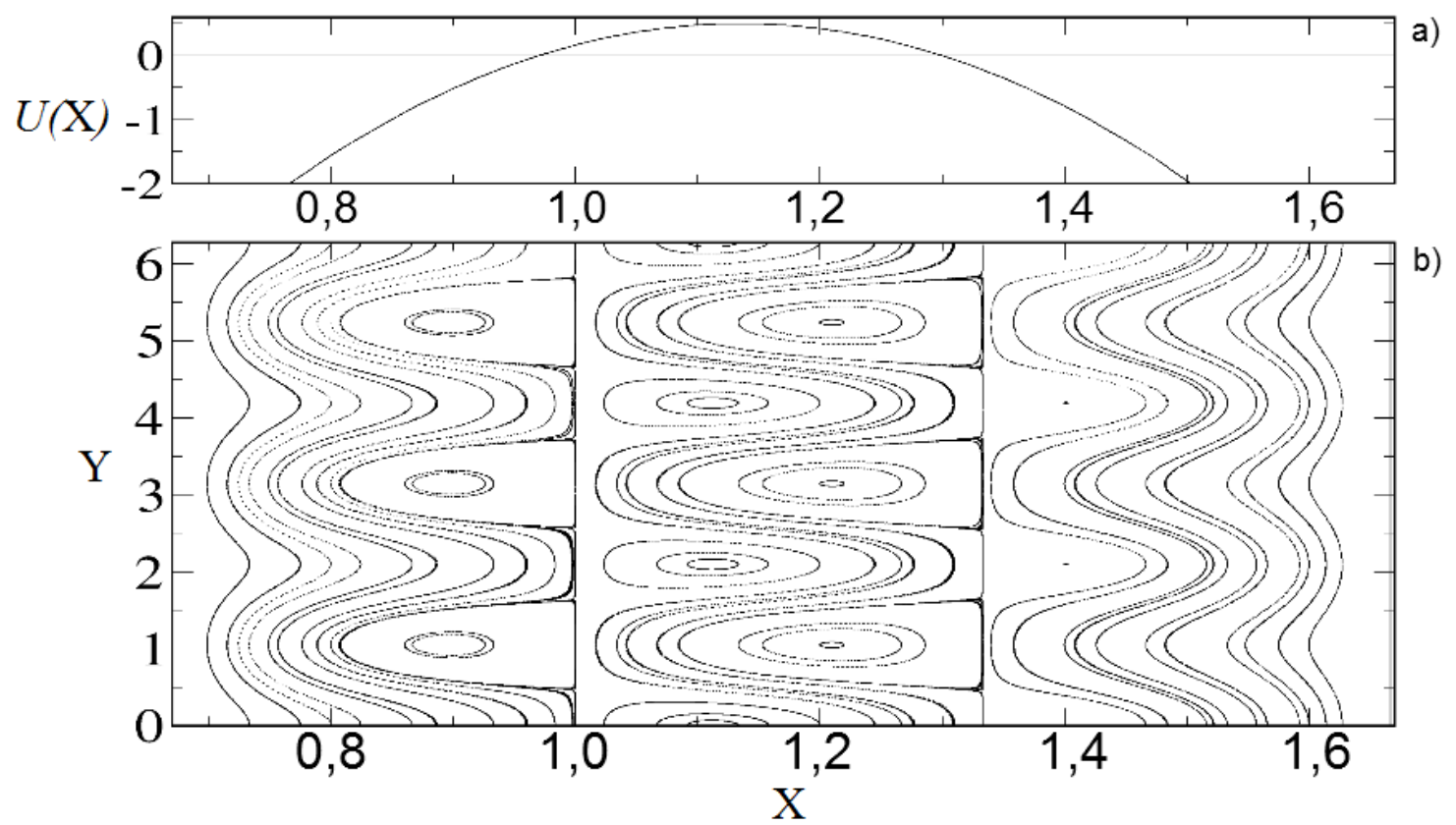

Figura 7.11: Seção de Poincaré do centro de guia das órbitas no espaço de fase (b) do sistema integrável de uma onda, eq. 7.7, cujo potencial eletrostático de equilíbrio, $\phi_{0}(x)$, está ligado ao perfil não monotônico do parâmetro de confinamento $U(X)$ (a). Nesta figura, $\mathrm{X}$ e Y estão ligados, respectivamente, as coordenadas radial e vertical normalizadas.

\subsection{Considerações finais acerca do controle do transporte radial de partículas}

Neste capítulo o transporte radial turbulento de partículas foi analisado para descargas perturbadas por um potencial elétrico imposto externamente, bias. Como visto nos capítulos 5 e 6, valores negativos e positivos de bias modificam as características do plasma de forma distinta. Por causa disso, e pelo fato de que apenas para valores positivos de bias o perfil da velocidade de fluxo é não monotônico na região radial analisada, o transporte foi estudado separadamente para valores positivos e negativos de bias. 
Utilizando a análise descrita no capítulo 3, perfis radiais do transporte induzido pela turbulência eletrostática foram calculados e interpretados utilizando um modelo Hamiltoniano de transporte caótico.

No caso de valores negativos de bias, mostrou-se que, no caso do Texas Helimak, o transporte não é reduzido onde o cisalhamento da velocidade vertical do plasma é alto, como tem sido previsto teoricamente (TERRY, 2000). Por outro lado, nestas descargas os perfis de transporte apresentam seus valores máximos próximos às regiões em que a velocidade de deriva elétrica $(\boldsymbol{E} \times \boldsymbol{B})$ é aproximadamente igual à velocidade de fase da onda. Estas regiões de máximo foram interpretadas, utilizando um modelo Hamiltoniano de interação partícula onda, como sendo resultado de um efeito de ressonância que incrementa o transporte.

Para valores positivos de bias, os perfis de velocidade de fluxo vertical do plasma não são monotônicos, apresentando uma posição radial de máximo valor. Nestes casos, foi observado que os perfis de transporte apresentam valores mínimos nas mesmas regiões radiais em que o cisalhamento da velocidade é nulo (shearless region). Esta observação foi interpretada como uma evidência de barreira de transporte de sem cisalhamento, já prevista em sistemas com fluxos não monotônicos (reversed shear flow systems) (MARCUS, 2008-2). 


\section{CONCLUSÕES E PERSPECTIVAS}

A turbulência e o transporte turbulento de partículas na borda do plasma têm sido amplamente estudados nas últimas décadas por sua relação com o confinamento magnético do plasma em Tokamaks.

Recentemente, máquinas toroidais de plasma com geometria simples de linhas de campo magnético, chamadas de tórus magnetizado simples - SMT, têm sido utilizadas para o estudo da turbulência e do transporte. Isso devido às suas características de baixa densidade e temperatura que se assemelham às do plasma na região periférica do plasma (como a borda e o scrape-off-layer) dos Tokamaks, mas que permitem, neste caso, a utilização de grandes conjuntos de sondas de Langmuir como sistema de diagnóstico.

O Texas Helimak é umas destas máquinas toroidais de plasma básico. Ela possui, como diagnóstico principal, um grande conjunto de sondas Langmuir (mais de 700 sondas) além um espectroscópio capaz de medir o perfil radial médio da velocidade vertical do plasma e a capacidade de controlar o perfil radial do campo elétrico radial através da imposição de um potencial elétrico externo (bias). Essas características fazem do Texas Helimak uma máquina muito interessante para o controle da turbulência e do transporte, que é o assunto de estudado nesta tese.

Conforme descrito a seguir, as análises apresentadas nesta tese mostraram como o controle da turbulência e do transporte de partículas no plasma no Helimak ocorre através da variação do potencial elétrico externo.

Dados de corrente de saturação iônica foram utilizados para estudar as características da turbulência como função da posição radial e do valor do bias imposto. Esses dados foram analisados do ponto de vista da distribuição da potencia espectral, da função de distribuição de probabilidade dos dados e da sua recorrência. Essa análise revelou que, de acordo com o sinal do bias aplicado a turbulência têm características distintas.

Em descargas tomadas com valores positivos e nulos de bias as flutuações apresentam potência espectral total e largura espectral grandes. Essa largura aumenta para valores maiores de bias. Além disso, essas descargas possuem PDFs com longas caudas positivas, caracterizando uma turbulência desenvolvida e dominada por eventos extremos (bursts). A relação entre o intervalo de tempo entre bursts, e a probabilidade 
de ocorrência de bursts em função desse tempo, foi calculada e mostrou-se que essa distribuição obedece a uma curva exponencial, típica de eventos independentes. A análise destes eventos extremos deve ser aprofundada em um futuro trabalho.

Por outro, as descargas com bias negativos apresentam larguras espectrais menores, PDF mais próxima da Gaussiana e uma recorrência (calculada através do diagrama de recorrência) mais evidente. Para essas descargas a auto-bicoerência foi calculada e comparada com a largura de banda para mostrar que o valor da largura espectral pode estar diretamente relacionado ao acoplamento de modos.

Um modelo de quatro ondas acopladas (LASHMORE-DAVIES, 2005) que inclui o fluxo de plasma (zonal flow) foi utilizado para interpretar a dependência da largura espectral com o valor de bias e da posição radial para descargas com bias negativo. Para isso os parâmetros do modelo foram obtidos a partir de características do plasma medidas experimentalmente. Utilizando esse modelo, o tempo de interação entre as ondas, foi estimado e comparado com os valores obtidos de largura espectral. O que se observou é que valores altos de tempo de interação coincidem com valores baixos de largura espectral. A conjectura apresentada é que tempos longos de interação restringem a excitação de muitos modos e levam portanto a um espectro de potência mais estreito.

A partir de dados de corrente de saturação iônica e de potencial flutuante medidos na configuração de sonda tripla, os perfis de transporte turbulento de partículas, foram medidos como função do bias imposto. Esses perfis de transporte foram então comparados com os perfis da velocidade vertical do fluxo de plasma e seu cisalhamento.

Foi observado que, para valores negativos de bias, há máximos no transporte em posições radiais que dependem do valor do bias. Um modelo Hamiltoniano proposto por MARCUS (2008) foi utilizado para interpretar esses máximos como sendo resultado da ressonância, entre a onda de deriva e o fluxo do plasma, que aumenta o transporte quando as velocidades de fase da onda e do fluxo do plasma são iguais (TOUFEN, 2012).

Já para valores positivos de bias, a velocidade de fluxo do plasma apresenta um ponto de máximo no seu perfil radial e consequentemente um ponto de cisalhamento nulo. Nestes casos foi observado também que os perfis de transporte apresentam pontos de mínimo nas mesmas posições radiais onde o cisalhamento da velocidade do fluxo é nulo. O mesmo modelo Hamiltoniano proposto por MARCUS (2008) foi utilizado para 
interpretar esses mínimos no transporte como sendo causados por uma barreira sem cisalhamento (shearless) (TOUFEN, 2012).

Em conclusão, a análise da turbulência e do transporte no Texas Helimak revelou novos comportamentos que podem contribuir para a melhoria da compreensão da turbulência e sua relação com o acoplamento de ondas, bem como do transporte de partículas e sua redução associada ao aparecimento de barreiras na borda do plasma em máquinas de fusão.

Uma próxima etapa deste trabalho será o estudo mais aprofundado dos eventos extremos (bursts) identificados no plasma do Texas Helimak. A velocidade de propagação dos bursts nas direções vertical e radial, por exemplo, pode ser determinada para descargas com valores distintos de bias. Também pode ser calculado o perfil médio dos bursts, seu tempo de crescimento e relaxação, como função da posição e do bias.

Uma outra possibilidade é ampliar as investigações sobre transporte e a turbulência no Texas Helimak através de experiências nas quais o campo magnético vertical passa a ser uma variável de controle. Nestas experiências, realizadas no segundo semestre de 2011, esse campo foi modificado em diferentes descargas. Uma investigação semelhante a essa, mas que não levou em consideração a imposição de bias externo foi realizada recentemente no TORPEX (POLI, 2007). 


\section{REFERÊNCIAS}

ASAKURA N.; et. al.; Statistical analysis to the SOL plasma fluctuation in JT-60U. Journal of Nuclear Materials. v. 390-391, p. 364-367, 2009.

BARASHENKOV, I. V.; ZEMLYANAYA, E. V.; Stable Complexes of Parametrically Driven, Damped Nonlinear Schrödinger Solitons. Physical Review Letters. v. 83, p. 2568-2571, 1999.

BESPAMYATNOV, I. O.; ROWAN, W. L.; LIAO, K. T.; GRANETZ, R. S.; An integrated charge exchange recombination spectroscopy/beam emission spectroscopy diagnostic for Alcator C-Mod tokamak. Review of Scientific Instruments. v. 81, p. 10D709(1-4), 2010.

BIGLARI, H.; DIAMOND P. H.; TERRY, P. W.; Influence of sheared poloidal rotation on edge turbulence. Physics Fluids B. v.2 p.1-4, 1990.

BITTENCOURT, J. A. Fundamentals of Plasma Physics. Oxford: Pergamon Press, 1986.

CAIRNS, R. A.; Radio Frequency Heating of Plasmas. Bristol: Adam Hilger, 1991.

DEL-CASTILLO-NEGRETE, Diego; Chaotic transport in zonal flows in analogous geophysical and plasma systems. Physics of Plasma. v. 7, n. 5, p. 1702-1711, 2000.

CARTER, T. A.; MAGGS, E.; Modifications of turbulence and turbulent transport associated with a bias-induced confinement transition in the Large Plasma Device. Physics of Plasma. v. 16, p. 012304(1-9), 2009.

CASTRO, R. M.; Turbulência Eletrostática e Magnética em Tokamaks. (Doutorado em Ciências) - Instituto de Física da Universidade de São Paulo, 1996.

CHARNEY, Jule G.; Geostrophic Turbulence. Journal of Atmospheric Science. v. 28 p. 1087-1095, 1971.

CHEN, F. F.; Introduction to Plasma Physics and Controlled Fusion. New York: Plenum Press, 1984.

CONWAY, G. D.; Turbulence measurements in fusion plasmas. Plasma Physics and Controlled Fusion. v. 50, p. 124026(1-11), 2008.

DAHLBURG, R. B.; et. al. Evolution of the bounded magnetized jet and comparison with Helimak experiments. Physics of Plasma. v. 16, n. 5, p. 072109(1-14), 2009.

DENDY, R. O.; CHAPMAN S. C.; Characterization and interpretation of strongly nonlinear phenomena in fusion, space and astrophysical plasmas. Plasma Physics and Controlled Fusion. v. 48, p. B313-B328, 2006. 
FERREIRA, A. A.; Turbulência em um plasma toroidal medida por sondas eletrostáticas. (Mestrado em Ciências) - Instituto de Física da Universidade de São Paulo, 1999.

FERREIRA, A. A.; HELlER, M. V. A. P.; CALDAS, I. L.; et. al.; Experimental analysis of mode coupling and plasma turbulence induced by magnetic fields. Physics of Plasma. v. 7, p. 3567(1-6), 2000.

FERREIRA A. A.; et. al.; Turbulence and transport in the scrape-off layer TCABR tokamak. Plasma Physics and Controlled Fusion. v. 46, p. 669-679, 2004.

FASOLI, A; et. al.; Electrostatic turbulence and transport in a simple magnetized plasma. Physics of Plasma. v. 13, p. 055902(1-9), 2006.

FREIDBERG, J. P. Plasma Physics and Fusion Energy. Cambridge: Cambridge University Press, 2007.

GARBET, X.; Turbulence in fusion plasmas: key issues and impact on transport modeling. Plasma Physics and Controlled Fusion. v. 43, p. A251-A266, 2001.

GARBET, X.; et. al. Physics of transport in tokamaks. Plasma Physics and Controlled Fusion. v. 46, p. B557-B574, 2004.

GALVÃO, R. M. O.; et. al. New regime of runaway discharges in tokamaks. Plasma Physics and Controlled Fusion. v. 43, p. 1181-1190, 2001.

GEKELMAN, W.; et. al. Design, Construction and Properties of the Large Plasma Research Device - The LAPD at UCLA, Review of Scientific Instruments, 62, 28752883, 1991.

GENTLE, K. W. et. Al. Transport studies in the TEXT tokamak. Plasma Physics and Controlled Fusion. v. 26, n. 12A, p. 1407-1418, 1984.

GENTLE, K. W.; HUANG He; Texas Helimak. Plasma Science and Technology. v. 10, n. 3, p. 1-6, 2008.

GENTLE, K. W.; LIAO, K.; LEE, K.; ROWAN, W. L.; Comparison of Velocity Shear with Turbulence Reduction Driven by Biasing in a Simple Cylindrical Slab Plasma. Plasma Science and Technology. v. 12, n. 4, p. 391-396, 2010.

GONÇALVES, B. et al. Role of Turbulence on Edge Momentum Redistribution in the TJ-II Stellarator. Physical Review Letters. v. 96, p. 145001(1-4), 2006.

GUIMARÃES-FILHO, Z. O.; et. al. Electrostatic turbulence driven by high magnetohydrodynamic activity in Tokamak Chauffage Alfvén Brésilien. Physics of Plasma. v. 15, p. 062501(1-8), 2008.

GUIMARÃES-FILHO, Z. O.; et. al. Recurrence quantification analysis of electrostatic fluctuations in fusion plasmas. Physics Letters A. v. 372, p. 1088-1095, 2008-2. 
GUIMARÃES-FILHO, Z. O.; et. al. Recurrence quantification analysis of turbulent fluctuations in the plasma edge of Tokamak Chauffage Alfvén Brésilien tokamak Physics of Plasma. v. 17, p. 012303(1-8), 2010.

HASEGAWA, A; MIMA, K. Pseudo-three-dimensional turbulence in magnetized nonuniform plasma. Physics of Fluids. v. 21, p. 87-92, 1978.

HASEGAWA, A; McCLENNAN C. G.; KODAMA, Y. Nonlinear behavior and turbulence spectra of drift waves and Rossby waves. Physics of Fluids. v. 22, p. 21222129, 1979.

HAZELTINE, R. D.; PRAGER, S. C.; New Physics in Fusion Plasma Confinement. Physics Today. v. 55, n.7, p. 30-35, 2002.

HELLER, M. V. A. P.; et. al. Edge turbulence spectrum alterations driven by resonant fields. Nuclear Fusion, v. 35, p. 59-67, 1995.

HELLER, M. V. A. P.; et. al. Scrape-Off Layer Turbulence Modulated by Mirnov Oscillations. Czechoslovak Journal of Physics, v. 55, p. 265-270, 2005.

HELLER, M. V. A. P.; et. al. Tokamak Turbulence at Scrape-off Layer in TCABR with Ergodic Magnetic Limiter. Journal of Plasma Physics, v. 73, p. 295-306, 2007.

HIDALGO, C.; et. al. Experimental Evidence of Three-Wave Coupling on Plasma Turbulence. Physical Review Letters. v. 71, n. 19, p. 3127-3130, 1993.

HIDALGO, C.; et. al.; Edge Turbulence and Anomalous 'Transport in Fusion Plasmas. Plasma Physics and Controlled Fusion. v. 37, p. A53-A57, 1995.

HIDALGO, C.; et. al. Experimental evidence of fluctuations and flows near marginal stability and dynamical interplay between gradients and transport in the JET plasma boundary region. Jornal Of Nuclear Materials. v. 313 - 316, p. 863-867, 2003.

HIDALGO, C.; On the nature of the transport in fusion plasmas. Astrophysics and Space Science. v. 292. p. 681-690, 2004.

HIDALGO, C.; et. al. Overview of TJ-II experiments. Nuclear Fusion. v. 45 p. S266S275, 2005.

HORTON, W.; Anomalous ion conduction from toroidal drift modes. Plasma Physics. V. 23, n. 12, p. 1107, 1981.

HORTON, W.; Drift waves and transport. Review of Modern Physics. v. 71, n. 3, p. 735-778, 1999.

HORTON, W.; et. al. Vorticity probes and the characterization of vortices in the Kelvin-Helmholtz instability in the large plasma device experiment. Physics of Plasma. v. 12, p. 022303(1-8), 2005. 
KIM, Y. C.; POWERS, E. J.; Digital bispectral analysis of self excited fluctuation spectra. Physics of Fluids. v. 21, p. 1452-1453, 1978.

KIM, Y. C.; BEALL, J. M., POWERS, E. J.; MIKSAD, R. W.; Bispectrum and nonlinear wave coupling. Physics of Fluids. v. 23, p. 258, 1980.

KRALL, N. A. Drift Waves. In Advances in Plasma Physics. v. 1. Edited by SIMON, A. and THOMPSON, W. B. New York: Interscience Publishers, 1968.

KURTHS, J. et al. Testing for nonlinearity in radiocarbon data. Nonlinear Processes in Geophysics. v.1 p. 72-75, 1994.

KUZNETSOV, Y. A. Elements of Applied Bifurcation Theory. New York: SpringerVerlag, 2004.

LABIT B.; et. al.; Universal Statistical Properties of Drift-Interchange Turbulence in TORPEX Plasmas. Physical Review Letters. v. 98, p. 255002(1-4), 2007.

LASHMORE-DAVIES, C. N.;, Modulational instability of a finite amplitude Alfvén wave. Physics of Fluids. v. 19, p. 587-589, 1976.

LASHMORE-DAVIES, THYAGARAJA, A.; C. N.; CAIRNS, R. A.; The effect of nonlinear mode coupling on the stability of toroidal Alfvén eigenmodes. Physics of Plasma. v. 4, p. 3243-3253, 1997.

LASHMORE-DAVIES, C. N.; McCARTHY, D. R.; THYAGARAJA, A.; The nonlinear dynamics of the modulational instability of drift waves and the associated zonal flows. Physics of Plasma. v. 8, p. 5121-5133, 2001.

LASHMORE-DAVIES, C. N.; THYAGARAJA, A.; McCARTHY, D. R.; Spectral transfers and zonal flow dynamics in the generalized Charney-Hasegawa-Mima model. Physics of Plasma. v. 12, p. 122304(1-12), 2005.

LI, B.; et. al. Plasma transport and turbulence in the Helimak: Simulation and experiment. Physics of Plasma. v. 16, p. 082510(1-7), 2009.

LI, B.; et. al. Turbulence and bias-induced flows in simple magnetized toroidal plasmas. Physcial Review E. v. 83, p.056406 (1-6), 2011.

LOPES, S. R.; et al; Deterministic signature for intermittent convective transport in plasma turbulence. Submetido a Physical Review Letters. 2012. Disponível em < http://fisica.ufpr.br/viana/artigos/submitted>. Acesso em: 07 ago 2012.

LIN, HONG.; Turbulence and Transport studies in the edge plasma of the TEXT tokamak. (Doctor of Philosophy) - The University of Texas at Austin, 1991.

LUCKHARDT, S.; The Helimak: A one dimensional toroidal plasma system. Technical report. San Diego: University of California, 1999. Disponível em: < http://orion.ph.utexas.edu/ starpower/ >. Acesso em: 13 jan 2012. 
MARCH, T. K.; CHAPMAN S. C.; DENDY, R. O.; Mutual information between geomagnetic indices and the solar wind as seen by WIND: Implications for propagation time estimates. Geophysical Research Letters. v. 32, p. L04101(1-4), 2005.

MARCUS, F. A.; Transporte de partículas induzido por ondas de deriva. (Doutorado em Física) - Instituto de Física da Universidade de São Paulo, 2004.

MARCUS, F. A., et. al. Reduction of chaotic particle transport driven by drift waves in sheared flows. Physics of Plasma. v. 15, p. 102304(1-9), 2008.

MARCUS, F. A., et. al. Chaotic transport in reversed shear tokamaks. Nuclear Fusion. v. 48, p. 024018(1-8), 2005.

MARWAN, N., KURTHZ, J.; Nonlinear analysis of bivariate data with cross recurrence plots. Physics Letters A. v. 302, p. 299-307, 2002.

MARWAN, N. et al. Comparing modern and Pleistocene ENSO-like influences in NW Argentina using nonlinear time series analysis methods. Climate Dynamics. v. 21, p. 317-326, 2003.

MARWAN, N.; et al. Recurrence plots for the analysis of complex systems. Physics Reports. v. 438, p. 237-329, 2007.

MEADE, D.; 50 years of fusion research. Nuclear Fusion. v. 50, p. 014004(1-14), 2010.

MOTOJIMA, O. et. al.; Initial physics achievements of large helical device experiments. Physics of Plasma. v. 6, p. 1843-1850, 1999.

MOTT-SMITH, H. M.; LANGMUIR, I.; The Theory of Collectors in Gaseous Discharges. Physical Review. v. 28, p. 727, 1926.

MÜLLER, S. H.; et. al.; Effects of a Vertical Magnetic Field on Particle Confinement in a Magnetized Plasma Torus. Physical Review Letters. v. 93, n. 16, p. 165003(14), 2004.

MÜLLER, S. H.; et. al.; Basic turbulence studies on toroidal plasma experiment and challenges in the theory-experiment comparison. Physics of Plasma. v. 12, p. 1, 2005.

NASCIMENTO, I. C.; et. al. Plasma confinement using biased electrode in the TCABR tokamak. Nuclear Fusion. v. 45, p. 796-803, 2005.

NASCIMENTO, I. C.; et. al.; Suppression and excitation of MHD activity with an electrically polarized electrode at the TCABR tokamak plasma edge. Nuclear Fusion. v. 47, p. 1570-1576, 2007.

NIST/SEMATECH e-Handbook of Statistical Methods, 2012. Disponível em: <http://www.itl.nist.gov/div898/handbook/eda/section3/eda35b.htm>, acesso em 11/09/2012. 
PEREZ, J. C.; et. al.; Drift wave instability in the Helimak experiment. Physics of Plasma. v. 13, p. 032101(1-10), 2006.

PITTS, R.; BUTTERY, R.; PINCHES, S.; Fusion: The way ahead, Physics World, v. 19, p. 20, 2006.

POLI, F. M.; Electrostatic instabilities and turbulence in a toroidal magnetized plasma. (Ph.D. thesis, no. 3849) - École Polytechnique Fédérale de Lausanne, Switzerland, 2007. Disponível em: < http://dx.doi.org/10.5075/epfl-thesis-3849>.

RICCI, P.; ROGERS, B. N.; BRUNNER, S.; High- and Low-Confinement Modes in Simple Magnetized Toroidal Plasmas. Physical Review Letters. v. 100, p. 225002(14), 2008.

RICCI, P.; ROGERS, B. N.; Three-dimensional fluid simulations of a simple magnetized toroidal plasma. Physics of Plasma. v. 16, p. 092307, 2009.

RITZ, Ch. P.; et. al.; Advanced plasma fluctuation analysis techniques and their impact on fusion research(invited). Review of Scientific Instruments. v. 59, p. 1739-1744, 1988.

ROWAN, W. L.; BESPAMYATNOV, I. O.; GRANETZ, R. S.; Wide-view charge exchange recombination spectroscopy diagnostic for Alcator C-Mod. Review of Scientific Instruments. v. 79, p. 10F529(1-4), 2008.

RYPDAL, K.; RATYNSKAIA, S.; Fluctuation threshold and profile resilience in weakly ionized plasma in a curved, unsheared magnetic field. Physics of Plasma. v. 11, p. 4623, 2004.

RYPDAL, K.; RATYNSKAIA, S.; Onset of Turbulence and Profile Resilience in the Helimak Configuration. Physical Review Letters. v. 94, p. $225002(1-4), 2005$.

DOS SANTOS LIMA, G. Z.; Análise da Turbulência do Plasma no Tokamak TCABR. (Doutorado em Física) - Instituto de Física da Universidade de São Paulo, 2009.

DOS SANTOS LIMA, G. Z.; et. al.; Bicoherence in electrostatic turbulence driven by high magnetohydrodynamic activity in Tokamak Chauffage Alfvén Brésilien, Physics of Plasmas, v. 16,p. 042508(1-10), 2009.

DA SILVA, R. P., NASCIMENTO, I. C.; DA CRUZ, D. F.; Electrostatic Ion Probe for Tokamak-Plasma-Edge Diagnostic, Review of Scientific Instruments, v. 57, p. 2205-2211, 1986.

DA SILVA, R. P., NASCIMENTO, I. C.; Simultaneous measurement of ion and electron temperatures in the scrape-off layer of a small tokamak, Review of Scientific Instruments, v. 62, p. 2700-2709, 1991.

SOMMERIA, J.; MEYERS, S. D.; SWINNEY, H. L.; Laboratory model of a planetary eastward jet. Nature. v. 337, p. 58-61, 1989. 
SOLOMON, T.; HOLLOWAY, W.; SWINNEY, H.; Shear flow instabilities and Rossby waves in barotropic flow in a rotating annulus. Physics Fluids A. v.5 p.1971 (112), 1993.

STILLERMAN, J. A.; FREDIAN, T. W.; KLARE, K. A.; MANDUCHI, G.; MDSplus data acquisition system. Review of Scientifc Instruments, v. 68, n. 1, p. 939-942, 1997.

SWOLYAKOV, A; DIAMOND, P. H.; SHEVCHENKO, V. I.; Zonal flow generation by parametric instability in magnetized plasmas and geostrophic fluids. Physics of Plasma. v. 7, p. 1349-1351, 2000.

TAYLER, R. J.; Necessary and sufficient conditions for the hydromagnetic RayleighTaylor stability of a cylindrical plasma. Journal of Nuclear Energy, Part C Plasma Physics. v. 3, p. 266-272, 1961.

TERRY, P. W.; Suppression of turbulence and transport by sheared flow. Review of Modern Physics. v. 72, n. 1, p. 109-165, 2000.

THEILER, C.; Basic Investigation of Turbulent Structures and Blobs of Relevance for Magnetic Fusion Plasmas (Ph.D. thesis, no. 5228) - École Polytechnique Fédérale de Lausanne, Switzerland, 2011. Disponível em: < http://dx.doi.org/10.5075/epfl-thesis5228>.

TOUFEN, D. L. et. al. Turbulence driven particle transport in Texas Helimak. Physics of Plasma. v. 19, p. 012307(1-8), 2012.

TOUFEN, D. L. at. al. Analysis of the Influence of External Biasing on Texas Helimak Turbulence. Physics of Plasma. artigo submetido. 2012-2.

VAN MILLIGEN, B. Ph.; et. al. Wavelet bicoherence: A new turbulence analysis tool. Physics of Plasma. v. 2, p. 3017-3032, 1995.

VAN MILligEN, B. Ph.; KALHOFF, T.; PEDROSA, M. A.; HIDALGO, C.; Bicoherence during confinement transitions in the TJ-II stellarator. Nuclear Fusion. v. 48, p. 115003(1-9), 2008.

VAN OOST, G.; et. al.; Turbulent transport reduction by $E \times B$ velocity shear during edge plasma biasing: recent experimental results. Plasma Physics and Controlled Fusion. v. 45, p. 621-643, 2003.

ZIMMERMAN, E. D.; LUCKHARDT, S. C.; Measurement of the Correlation Spectrum of Electrostatic Potential Fluctuations in an ECRH Helimak Plasma. Jornal of Fusion Energy. v. 12, p. 289, 1993. 


\section{LISTA DE FIGURAS}

Figura 2.1: Desenho esquemático do Texas Helimak mostrando um exemplo de linha de campo magnético em azul e os quatro conjuntos de placas de bias no topo e fundo da máquina. As placas utilizadas para a aplicação de potencial externo (bias) nas experiências analisadas estão representadas em amarelo.

Figura 2.2: Foto do Texas Helimak mostrando o vaso de aço inox, os conjuntos de bobinas toroidais em vermelho e das bobinas verticais em azul.

Figura 2.3: Foto de uma das placas de bias, indicadas na Figura 2.1, mostrando as sondas de Langmuir distribuídas na superfície da placa.

Figura 2.4: Foto de um dos quatro conjuntos de quatro placas de bias, indicadas na Figura 2.1, montadas no fundo do vaso do Texas Helimak. (fonte: GENTLE, K. W.; disponível em < http://www.ph.utexas.edu/ phy315>) ..... 19

Figura 2.5: Exemplo de quatros medidas de corrente de saturação iônica no mesmo disparo utilizando quatro sondas posicionadas em quatro raios diferentes e na mesma posição vertical $(\mathrm{z}=1,747 \mathrm{~m})$.

Figura 2.6: Arranjo experimental utilizado para medidas espectroscópicas de velocidade de fluxo do plasma $(V z)$.

Figura 2.7: Espectrômetro utilizado para medidas de velocidade de fluxo do plasma $(V z)$

Figura 2.8: Perfil radial experimental de densidade volumétrica de partículas do

Texas Helimak medido em $\mathrm{z}=1,747$ e em disparos com bias nulo.

Figura 2.9: Evolução temporal do bias e da corrente de saturação iônica em um disparo em que o bias muda ao longo do tempo. Observa-se o efeito do valor do bias sobre a turbulência pois para valores mais negativos do bias a amplitude da turbulência é reduzida. Neste caso, $\mathrm{I}_{\mathrm{sat}}$ foi medida em uma sonda posicionada em $\mathrm{R}=1,110 \mathrm{~m} \mathrm{e} \mathrm{z}=1,747 \mathrm{~m}$.

Figura 3.1: Perfis radiais de quatro grandezas estatísticas (média, nível de turbulência, skewness e kurtosis) da corrente de saturação iônica para z = 1,747 m. As linhas pontilhadas vermelhas marcam os valores de skewness e kurtosis da distribuição gaussiana. 
Figura 3.2: Exemplo de sinal de corrente de saturação iônica em função do tempo (acima) e espectro de potência (abaixo). Neste exemplo a sonda estava localizada em $\mathrm{R}=1,210 \mathrm{~m} \mathrm{e} \mathrm{z}=1,747 \mathrm{~m}$.

Figura 3.3: Exemplos de números de onda médios em função da frequência, $k f$, para pares de sondas medindo corrente de saturação iônica. Neste exemplo fixou-se uma sonda $(\mathrm{R}=1,210 \mathrm{~m}$ e $\mathrm{z}=1,747 \mathrm{~m})$ e foram utilizadas outras quatro sondas com a mesma posição radial $(\mathrm{R}=1,210 \mathrm{~m})$ e diferentes alturas $(\mathrm{z}=1,767 \mathrm{~m}, 1,787 \mathrm{~m}, 1,807 \mathrm{~m}$ e $1,827 \mathrm{~m})$. Nos espectros para $\Delta \mathrm{z}$ $=6 \mathrm{~cm}$ e $8 \mathrm{~cm}$ foi utilizada uma correção para pontos com $\theta f>2 \pi$ fossem posicionados na mesma reta.

Figura 3.4: Exemplo de espectro de $S(k, f)$ para duas sondas medindo corrente de saturação iônica. A reta em preto representa a velocidade de fase estimada (neste exemplo: $V p h \cong-100 \mathrm{~m} / \mathrm{s}$ ). Neste exemplo as sondas estavam localizadas no mesmo raio $(\mathrm{R}=1,210 \mathrm{~m})$ e diferentes valores de $\mathrm{z}(\mathrm{z}=$ $1,747 \mathrm{~m} \mathrm{e} \mathrm{z}=1,767 \mathrm{~m})$.

Figura 3.5: Exemplo de espectros de coerência para pares de sondas medindo corrente de saturação iônica. Neste exemplo fixou-se uma sonda $(\mathrm{R}=$ $1,210 \mathrm{~m} \mathrm{e} \mathrm{z}=1,747 \mathrm{~m}$ ) e foram utilizadas outras quatro sondas com a mesma posição radial $(\mathrm{R}=1,210 \mathrm{~m})$ e diferentes alturas $(\mathrm{z}=1,767 \mathrm{~m}$, $1,787 \mathrm{~m}, 1,807 \mathrm{~m}$ e $1,827 \mathrm{~m})$

Figura 3.6: Exemplo de espectro de auto-bicoerência para o sinal de corrente de saturação iônica medido pela sonda localizada em $\mathrm{R}=1,210 \mathrm{~m} \mathrm{e} \mathrm{z}=$

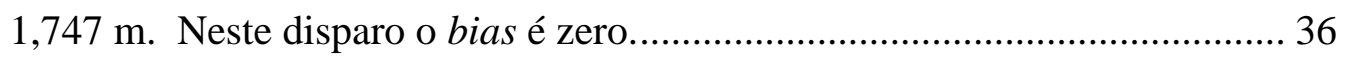

Figura 3.7: Curva característica de uma sonda de Langmuir. ........................................ 38

Figura 3.8: Curva característica da sonda de Langmuir (corrente em função do potencial na sonda) ajustada aos dados do Texas Helimak para raio $\mathrm{R}=$ $0,975 \mathrm{~m}$.

Figura 3.9: Exemplos de diagramas de recorrência para (a) um sinal senoidal e (b) um sinal aleatório.

Figura 3.10: Exemplo de diagrama de recorrência para um série de corrente de saturação iônica medida em uma sonda posicionada em $\mathrm{R}=1,190, \mathrm{z}=$ 1,747 e disparo com valor de bias igual a zero. Para esse diagrama o valor do determinismo estimado foi DET $=0,97(1)$. 
Figura 3.11: Exemplo de perfil radial de velocidade vertical de fluxo do plasma, $V z$, e de perfil radial do cisalhamento (shear), $d V z / d R$

Figura 4.1: Perfis radiais de corrente de saturação média (a), potencial flutuante médio (b) e temperatura média (c).

Figura 4.2: Valores médios de corrente de saturação iônica em função do raio $(R)$ e da posição vertical (z). Nesta figura apenas está mostrada a faixa radial de maior interesse: $1,10 \mathrm{~m}<\mathrm{R}<1,25 \mathrm{~m}$.

Figura 4.3: Perfis radiais aproximados de nível de turbulência da densidade (azul) e do potencial do plasma (vermelho), as curvas representam a tendência dos dados. Nota-se que as duas curvas estão mais próxima na faixa radial $1,10 \mathrm{~m}<\mathrm{R}<1,25 \mathrm{~m}$, mostrando que nesta faixa a relação de adiabaticidade pode ser considerada satisfeita.

Figura 4.4: Distribuição de sondas utilizada para coletar os dados da análise espectral.

Figura 4.5: Espectro de potência, Pxx, de corrente de saturação iônica para quatro sondas posicionadas em quatro raios distintos e mesma altura $(\mathrm{z}=$ $1,747 \mathrm{~m})$.

Figura 4.6: Espectrogramas de potência, Pxx, de corrente de saturação iônica para quatro sondas posicionadas em quatro raios distintos e mesma altura $(\mathrm{z}=$ $1,747 \mathrm{~m})$

Figura 4.7: Espectro de potência, Pxx, em função da posição radial.

Figura 4.8: Espectros de $k(f)$ em função do raio no qual o valor de $k(f)$ esta representado pela escala de cor (a) e dois espectros de $k(f)$ para dois valores de raio, $\mathrm{R}=1,110 \mathrm{~m}$ (b) e $\mathrm{R}=1,170 \mathrm{~m}$ (c), ilustrando a relação linear entre o número de onda e a frequência. Esses espectros mostram que o sentido da velocidade de fase não se inverte na região radial $1,10 \mathrm{~m}<\mathrm{R}<$ $1,25 \mathrm{~m}$.

Figura 4.9: Espectro de $S(k, f)$ para raio $\mathrm{R}=1,170 \mathrm{~m}$. A reta em preto ilustra a velocidade de fase média estimada. Nota-se que a velocidade de fase média estimada é uma boa aproximação para a região de maior potência do espectro $(f \leq 2 \mathrm{kHz})$.

Figura 4.10: Velocidade de fase $\left(\mathrm{V}_{\mathrm{ph}}\right)$ estimada através do espectro de $S(k, f)$ em função da posição radial. 
Figura 5.1: Perfis radiais médios de corrente de saturação iônica para cinco valores distintos de bias $(-20 \mathrm{~V},-8 \mathrm{~V}, 0,+8 \mathrm{~V} \mathrm{e}+20 \mathrm{~V})$. Todas as medidas foram feitas com sondas posicionadas na mesma posição vertical da máquina (z $=1,747 \mathrm{~m})$.

Figura 5.2: Corrente de saturação iônica média como função da posição radial da sonda e do valor de bias aplicado. As linhas pontilhas marcam a região radial onde esta localizada a placa de bias. Todas as medidas foram feitas com sondas posicionadas na mesma posição vertical da máquina $(\mathrm{z}=$ $1,747 \mathrm{~m})$

Figura 5.3: Perfis radiais médios de potencial flutuante para cinco valores distintos de bias $(-20 \mathrm{~V},-8 \mathrm{~V}, 0,+8 \mathrm{~V}$ e $+20 \mathrm{~V})$. Todas as medidas foram feitas com sondas posicionadas na mesma posição vertical $\mathrm{z}=1,747 \mathrm{~m}$.

Figura 5.4: Potencial flutuante médio como função da posição radial da sonda e do valor de bias aplicado. As linhas pontilhas marcam a região radial onde esta localizada a placa de bias. Todas as medidas foram feitas com sondas posicionadas na mesma posição vertical $\mathrm{z}=1,747 \mathrm{~m}$.

Figura 5.5: Perfis radiais da temperatura eletrônica média para cinco valores distintos de bias $(-20 \mathrm{~V},-8 \mathrm{~V}, 0,+8 \mathrm{~V} \mathrm{e}+20 \mathrm{~V})$. Todas as medidas foram feitas com sondas posicionadas na mesma posição vertical $\mathrm{z}=1,747 \mathrm{~m}$.

Figura 5.6: Temperatura eletrônica média como função da posição radial da sonda e do valor de bias aplicado. As linhas pontilhas marcam a região radial onde esta localizada a placa de bias. Todas as medidas foram feitas com sondas posicionadas na mesma posição vertical $\mathrm{z}=1,747 \mathrm{~m}$.

Figura 5.7: Nível de turbulência para corrente de saturação iônica (a) e potencial flutuante (b) como função da posição radial da sonda e do valor de bias aplicado. As linhas pontilhadas vermelhas marcam a região radial onde esta localizada a placa de bias. Todas as medidas foram feitas com sondas posicionadas na mesma posição vertical $\mathrm{z}=1,747 \mathrm{~m}$.

Figura 5.8: Condição de adiabaticidade, equação 5.1, como função da posição radial da sonda e do valor de bias aplicado. As linhas pontilhadas marcam a região radial onde esta localizada a placa de bias. A escala de cor foi saturada em dois de modo que as regiões mais claras indiquem onde a condição de adiabaticidade é uma boa aproximação. Todas as medidas 
foram feitas com sondas posicionadas na mesma posição vertical $\mathrm{z}=$ $1,747 \mathrm{~m}$.

Figura 5.9: Espectros de potência, Pxx, em função do raio R para seis disparos com valores de bias zero ou positivos. Nesta figura a potência esta representada pela cor. Em todas as sondas o valor da altura é a mesma $(z=1,747 \mathrm{~m})$.

Figura 5.10: Espectros de potência, Pxx, em função do raio R para oito disparos com valores de bias negativos. Nesta figura a potência esta representada pela cor. Em todas as sondas o valor da altura é a mesma $(\mathrm{z}=1,747 \mathrm{~m})$.

Figura 5.11: Espectro de potência, a potência esta representada pela cor, em função do raio $\mathrm{R}$ para disparo com bias $=-35 \mathrm{~V}$ mostrando a presença de um modo de $f \cong 4 \mathrm{kHz}$ (a). Espectros de potência para dois raios específicos, $\mathrm{R}=1,110 \mathrm{~m}$ (b) e $\mathrm{R}=1,190 \mathrm{~m}$ (c) nos quais o modo de $f \cong 4 \mathrm{kHz}$ pode ser observado com mais detalhes. Em todas as sondas o valor da altura é a mesma $(\mathrm{z}=1,747 \mathrm{~m})$.

Figura 5.12: Espectros de $k(f)$ em função do raio para quatro valores distintos de bias $(+15 \mathrm{~V}, 0,-16 \mathrm{~V}$ e $-30 \mathrm{~V})$. Nesta figura o valor de $k(f)$ esta representado pela escala de cor. Nota-se que para bias $<0$ ocorre a inversão da velocidade de fase.

Figura 5.13: Velocidade de fase $\left(\mathrm{V}_{\mathrm{ph}}\right)$ estimada através do espectro de $S(k, f)$ em função da posição radial e do valor do bias. Nesta figura a inversão do sentido de $\mathrm{V}_{\text {ph }}$ pode ser identificada.

Figura 5.14: Ilustração do efeito do bias na velocidade de deriva elétrica $\mathbf{V} E$ (setas verdes) na região radial próxima a placa de bias (que está posicionada na região $0,863 \mathrm{~m} \leq \mathrm{R} \leq 1,075 \mathrm{~m}$ ).

Figura 5.15: Velocidade de fluxo do plasma $\left(\mathrm{V}_{\mathrm{z}}\right)$ medida utilizando técnicas espectroscópicas. Nota-se a mudança no sentido da velocidade para valores negativos de bias".

Figura 6.1: Largura espectral, $\Delta f$, dos sinais da flutuação de corrente de saturação iônica como função da posição radial e vertical (a) e largura espectral média como função da posição radial (b). O disparo mostrado foi adquirido para bias nulo.

Figura 6.2: Largura espectral média, $\Delta f$, dos sinais de corrente de saturação iônica como função da posição radial e do valor de bias. 
Figura 6.3: Potência espectral total dos sinais de corrente de saturação iônica como função da posição radial e do valor de bias. Essa potência representa o valor médio em relação à coordenada vertical.

Figura 6.4: Diagramas de recorrência para descargas com (a) bias $=-25 \mathrm{~V}$, (b) bias $=0 \mathrm{~V}$, (c) bias $=+15 \mathrm{~V}$ e mesma posição radial e vertical $(\mathrm{R}=1,170 \mathrm{~m} \mathrm{e} \mathrm{z}$ $=1,747 \mathrm{~m})$.

Figura 6.5: Valores médios (média em z e em R) da largura espectral, $\Delta f$, como função do valor do bias.

Figura 6.6: Funções de distribuição de probabilidade (PDF) dos sinais de corrente de saturação iônica para três valores de bias: -25 V (linha tracejada), nulo (linha cheia) e $+15 \mathrm{~V}$ (linha pontilhada). Para fins de comparação a PDF da Gaussiana também é mostrada (em vermelho).

Figura 6.7: Ilustração da determinação do intervalo de tempo entre eventos extremos $(\tau)$ em um trecho de sinal de corrente de saturação iônica normalizada. Os picos que foram considerados como eventos extremos estão marcados com x e o limite de amplitudes utilizado nesta decisão está representado por uma linha tracejada azul. Neste caso a medida foi realizada por uma sonda em $\mathrm{R}=1,170 \mathrm{~m} \mathrm{e} \mathrm{z}=1,807 \mathrm{~m}$ e em um disparo com bias $=+15 \mathrm{~V}$

Figura 6.8: Função de distribuição de probabilidade (PDF) dos intervalos de tempo entre bursts sucessivos, $P(\tau)$ para dados de corrente de saturação iônica com bias $=+15 \mathrm{~V}$ e sondas posicionadas em $\mathrm{R}=1,170 \mathrm{~m}$. A curva em vermelho é um ajuste exponencial.

Figura 6.9: Espectro de autobicoerência para o sinal de corrente de saturação iônica medida pela sonda localizada em $\mathrm{R}=1,210 \mathrm{~m} \mathrm{e} \mathrm{z}=1,747 \mathrm{~m}$. Neste disparo o valor do bias é de $-8 \mathrm{~V}$.

Figura 6.10: Média dos valores verticais da (a) auto-bicoerência soma, $S b$, e (b) largura espectral como função da posição radial para uma descarga com bias $=-25 \mathrm{~V}$. A incerteza de cada ponto foi estimada através do desvio padrão dos valores obtidos no conjunto de seis sondas distribuídas verticalmente. A linha tracejada vermelha indica a soma da autobicoerência para um sinal randômico. 
Figura 6.11: Ajuste senoidal aproximado do perfil de velocidade de fluxo vertical, $V z$, para obtenção do número de onda do zonal flow, $q$, necessário para o modelo de quatro ondas acopladas.

Figura 6.12: Dependência do tempo de interação com as condições do plasma: Evolução temporal da amplitude de uma das ondas de banda lateral $\left(a_{+}\right)$ para duas posições radiais diferentes : (a) $\mathrm{R}=1,110 \mathrm{~m}$ e (b) $\mathrm{R}=1,201 \mathrm{~m}$. Em ambos os casos a posição vertical da sonda $(\mathrm{z}=1,747 \mathrm{~m})$ e o valor do bias (- $25 \mathrm{~V})$ são os mesmos.

Figura 6.13: (a) Tempo de interação médio e (b) largura espectral média, $\Delta f$, dos sinais de corrente de saturação iônica como função da posição radial e do valor de bias.

Figura 6.14: (a) Espectro de potência e (b) auto-bicoerência, mostrando o modo de $f=100 \mathrm{~Hz}$. Nota-se que este modo se acopla com todas as frequiências. Estes espectros correspondem a um disparo com bias $=-16 \mathrm{~V}$ e uma sonda posicionada em $(\mathrm{R}=1,210 \mathrm{~m} \mathrm{e} \mathrm{z}=1,747 \mathrm{~m})$.

Figura 6.15: Média dos valores verticais da (a) auto-bicoerência soma, $S b$, e (b) largura espectral como função da posição radial para uma descarga com bias $=-16 \mathrm{~V}$. A incerteza de cada ponto foi estimada através do desvio padrão dos valores obtidos no conjunto de seis sondas distribuídas verticalmente. A linha tracejada vermelha indica a soma da autobicoerência para um sinal randômico.

Figura 7.1: Distribuição de sondas utilizada para medidas de transporte turbulento..... 94

Figura 7.2: Perfis radiais do transporte turbulento de partículas, $\Gamma$, em função do bias, para valores negativos de bias.

Figura 7.3: Perfis radiais do Cisalhamento (shear) da velocidade vertical do fluxo de plasma para valores negativos de bias.

Figura 7.4: Espaços de fase do sistema integrável $(A 2=0)$ de uma onda, eq. 7.7, para $\mathrm{U}=0$ (a) e $\mathrm{U}=0,5$ (b). Nestes espaços de fase $X$ e $Y$ estão ligados, respectivamente, as coordenadas radial e vertical.

Figura 7.5: Mapa estroboscópico do sistema de duas ondas, eq. 7.1, para $U=0$ (a) e $\mathrm{U}=0,5$ (b). A relação da amplitudes das ondas nestes mapas é de $A 2$ / $A 1=0,01$. Nestes espaços de fase $X$ e $Y$ estão ligados, respectivamente, as coordenadas radial e vertical. 
Figura 7.6: Perfis radiais da diferença entre a velocidade de deriva elétrica, $V E$, e a velocidade de fase, $V p h$, em função de valores negativos de bias. Essa diferença é proporcional ao parâmetro de confinamento: $U$.

Figura 7.7: Perfil radial da velocidade vertical do plasma (a) e do cisalhamento (b) para bias $=+10 \mathrm{~V}$. Nestas curvas destaca-se a posição radial, $R *$, em que a velocidade apresenta um ponto de máximo e o cisalhamento é zero (ponto sem shear).

Figura 7.8: Perfis radiais do transporte turbulento de partículas, $\Gamma$, em função do bias, para valores positivos de bias.

Figura 7.9: Perfis radiais do cisalhamento (shear) da velocidade vertical do fluxo de plasma para valores positivos de bias.

Figura 7.10: Perfil radial da velocidade vertical do plasma (pontos pretos) e parábola ajustada (curva vermelha) para bias $=+10 \mathrm{~V}$

Figura 7.11: Seção de Poincaré do centro de guia das órbitas no espaço de fase (b) do sistema integrável de uma onda, eq. 7.7, cujo potencial eletrostático de equilíbrio, $\phi 0 x$, está ligado ao perfil não monotônico do parâmetro de confinamento $U(X)$ (a). Nesta figura, $\mathrm{X}$ e $\mathrm{Y}$ estão ligados, respectivamente, as coordenadas radial e vertical normalizadas. 


\section{APÊNDICE I: TABELA DE DISPAROS UTILIZADOS}

A tabela a seguir apresenta os disparos do Texas Helimak utilizados neste trabalho de doutorado.

\begin{tabular}{|c|c|c|}
\hline $\begin{array}{l}\text { Numero dos } \\
\text { disparos }\end{array}$ & Data & Tipo de medida \\
\hline $\begin{array}{l}110202006- \\
110202020\end{array}$ & $02 / 02 / 2011$ & $\begin{array}{l}\text { Distribuição radial e vertical de sondas medindo } \\
\text { corrente de saturação iônica. }\end{array}$ \\
\hline $\begin{array}{l}110216022- \\
110222040\end{array}$ & $\begin{array}{c}\text { De } \\
16 / 02 / 2011 \text { a } \\
22 / 02 / 2011 \\
\end{array}$ & $\begin{array}{l}\text { Medidas de perfil radial do transporte de partículas e } \\
\text { da temperatura eletrônica. }\end{array}$ \\
\hline $\begin{array}{c}907210003- \\
907210033\end{array}$ & $21 / 07 / 2009$ & $\begin{array}{l}\text { Medidas de perfil radial de corrente de saturação } \\
\text { iônica e da temperatura eletrônica. }\end{array}$ \\
\hline $\begin{array}{c}909100104- \\
909100120\end{array}$ & $10 / 09 / 2009$ & Medidas de perfil radial de potencial flutuante. \\
\hline $\begin{array}{l}908120050, \\
908120051 \\
908120073- \\
908120086 .\end{array}$ & $12 / 08 / 2009$ & Perfil radial de corrente de saturação iônica. \\
\hline 909080028 & 08/09/2009 & $\begin{array}{l}\text { Perfis radiais de potencial flutuante e corrente de } \\
\text { saturação iônica. Valor de bias variando em função do } \\
\text { tempo. }\end{array}$ \\
\hline $\begin{array}{l}910200008- \\
910200024\end{array}$ & $20 / 10 / 2009$ & $\begin{array}{l}\text { Perfis radiais de corrente de saturação iônica e } \\
\text { potencial flutuante para medida de transporte } \\
\text { turbulento. }\end{array}$ \\
\hline
\end{tabular}

